\title{
3D-FE Modeling of 316 SS under Strain-Controlled Fatigue Loading and CFD Simulation of PWR Surge Line
}

Interim Report on PWR Surge Line Material Testing, Thermal-Mechanical Stress Analysis, and Fatigue Evaluation

Nuclear Engineering Division 


\section{About Argonne National Laboratory}

Argonne is a U.S. Department of Energy laboratory managed by UChicago Argonne, LLC under contract DE-AC02-06CH11357. The Laboratory's main facility is outside Chicago, at 9700 South Cass Avenue, Argonne, Illinois 60439. For information about Argonne and its pioneering science and technology programs, see www.anl.gov.

\section{DOCUMENT AVAILABILITY}

Online Access: U.S. Department of Energy (DOE) reports produced after 1991 and a growing number of pre-1991 documents are available free via DOE's SciTech Connect (http://www.osti.gov/scitech/)

\section{Reports not in digital format may be purchased by the public from the National Technical Information Service (NTIS):}

U.S. Department of Commerce

National Technical Information Service

5301 Shawnee Rd

Alexandria, VA 22312

www.ntis.gov

Phone: (800) 553-NTIS (6847) or (703) 605-6000

Fax: (703) 605-6900

Email: orders@ntis.gov

\section{Reports not in digital format are available to DOE and DOE contractors from the Office of Scientific and Technical Information (OSTI):}

U.S. Department of Energy

Office of Scientific and Technical Information

P.O. Box 62

Oak Ridge, TN 37831-0062

www.osti.gov

Phone: (865) 576-8401

Fax: (865) 576-5728

Email: reports@osti.gov

\section{Disclaimer}

This report was prepared as an account of work sponsored by an agency of the United States Government. Neither the United States Government nor any agency thereof, nor UChicago Argonne, LLC, nor any of their employees or officers, makes any warranty, express or implied, or assumes any legal liability or responsibility for the accuracy, completeness, or usefulness of any information, apparatus, product, or process disclosed, or represents that its use would not infringe privately owned rights. Reference herein to any specific commercial product, process, or service by trade name, trademark, manufacturer, or otherwise, does not necessarily constitute or imply its endorsement, recommendation, or favoring by the United States Government or any agency thereof. The views and opinions of document authors expressed herein do not necessarily state or reflect those of the United States Government or any agency thereof, Argonne National Laboratory, or UChicago Argonne, LLC. 


\section{D-FE Modeling of 316 SS under Strain-Controlled Fatigue Loading and CFD Simulation of PWR Surge Line}

Interim Report on PWR Surge Line Material Testing, Thermal-Mechanical Stress Analysis, and Fatigue Evaluation

Subhasish Mohanty, Bipul Barua, Joseph Listwan, Saurin Majumdar, and Ken Natesan

Nuclear Engineering Division, Argonne National Laboratory

March 2017 
This page intentionally left blank 


\section{ABSTRACT}

In financial year 2017, we are focusing on developing a mechanistic fatigue model of surge line pipes for pressurized water reactors (PWRs). To that end, we plan to perform the following tasks: (1) conduct stress- and strain-controlled fatigue testing of surge-line base metal such as 316 stainless steel (SS) under constant, variable, and random fatigue loading, (2) develop cyclic plasticity material models of $316 \mathrm{SS}$, (3) develop one-dimensional (1D) analytical or closed-form model to validate the material models and to understand the mechanics associated with 316 SS cyclic hardening and/or softening, (4) develop three-dimensional (3D) finite element (FE) models with implementation of evolutionary cyclic plasticity, and (5) develop computational fluid dynamics (CFD) model for thermal stratification, thermal-mechanical stress, and fatigue of example reactor components, such as a PWR surge line under plant heat-up, cool-down, and normal operation with/without grid-load-following.

This semi-annual progress report presents the work completed on the above tasks for a 316 SS laboratory-scale specimen subjected to strain-controlled cyclic loading with constant, variable, and random amplitude. This is the first time that the accurate 3D-FE modeling of the specimen for its entire fatigue life, including the hardening and softening behavior, has been achieved. We anticipate that this work will pave the way for the development of a fully mechanistic-computer model that can be used for fatigue evaluation of safety-critical metallic components, which are traditionally evaluated by heavy reliance on time-consuming and costly test-based approaches. This basic research will not only help the nuclear reactor industry for fatigue evaluation of reactor components in a cost effective and less time-consuming way, but will also help other safety-related industries, such as aerospace, which is heavily dependent on test-based approaches, where a single full-scale fatigue test can cost millions of dollars and require years of effort to conduct.

Toward our goal of demonstration of fully mechanistic fatigue evaluation of reactor components, we also started work on developing a component-level computer model of reactor components, such as 316 SS surge line pipe. This requires developing a thermal-mechanical stress analysis model of the reactor surge line, which, in turn, requires time-dependent temperature and stratification information along the boundary of the pipe. Toward that goal, CFD models of surge lines are being developed. In this report, we also present some preliminary results showing the temperature conditions along the surge line wall under reactor heat-up, cool-down, and steadystate power operation. 
This page intentionally left blank 


\section{TABLE OF CONTENTS}

3D-FE Modeling of 316 SS under Strain-Controlled Fatigue Loading and CFD Simulation of PWR Surge Line

ABSTRACT

Table of Contents

List of Figures

List of TABLES

Abbreviations

Acknowledgments

1 Introduction

2 Theoretical Background on Evolutionary Cyclic Plasticity: APSE-Based 1D-Analytical and 3D-FE Modeling

2.1 Constitutive Relations for Evolutionary Cyclic Plasticity Model............................... 3

2.2 Time-Based Estimation of Time-Dependent Material Parameters .............................. 4

2.3 Modeling Fatigue Behavior using Evolutionary Cyclic Plasticity Model .................... 6

2.3.1 Time-Based Modeling 6

2.3.2 APSE-Based Modeling $\quad 7$

2.4 FE Implementation of Evolutionary Cyclic Plasticity Model................................... 8

3 Results from Strain Control Tensile/Fatigue Test and Material Parameter Estimation Models of 316 SS

3.1 Tensile Test and Associated Time/Cycle Independent Elastic-Plastic Material Model Results

3.2 Constant-Amplitude Fatigue Test and Associated Cyclic Elastic-Plastic Material

Model Results

3.3 Variable-Amplitude Fatigue Test and Associated Cyclic Elastic-Plastic Material

Model Results

3.4 Random-Amplitude Fatigue Test Results

4 Results from Analytical Modeling of Constant, Variable, and Random Load Fatigue Tests

4.1 Results from Time-Based Analytical Models

4.1.1 Constant-Amplitude Fatigue Test

4.1.2 Variable-Amplitude Fatigue Test

4.2 Results from Accumulated Plastic Strain Energy (APSE)-Based Analytical

Models .34

4.2.1 Constant-Amplitude Fatigue Test 34

4.2.2 Variable-Amplitude Fatigue Test 36

4.2.3 Random-Amplitude Fatigue Test 36 


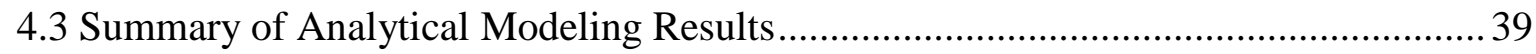

5 Results from FE Modeling 42

5.1 Results from Time-Based 3D-FE Models.................................................................. 44

5.1.1 Constant-Amplitude Fatigue Test 44

5.1.2 Variable-Amplitude Fatigue Test 48

5.2 Results from APSE-Based 3D-FE Models ............................................................... 51

5.2.1 Constant-Amplitude Fatigue Test 51

5.2.2 Variable-Amplitude Fatigue Test 53

5.2.3 Random-Amplitude Fatigue Test 54

5.3 Summary of FE Modeling Results and Comparison with Analytical Modeling

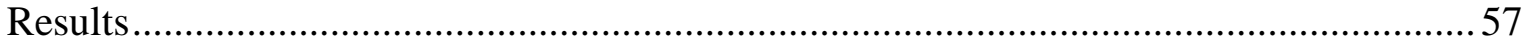

6 Computational Fluid Dynamics Simulation of Surge Line Pipe for Thermal Stratification Evaluation $\quad 64$

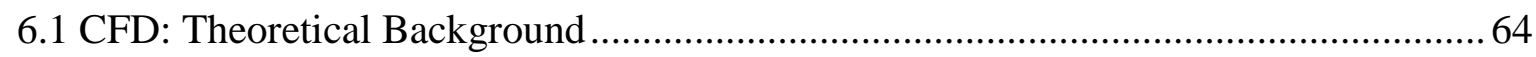

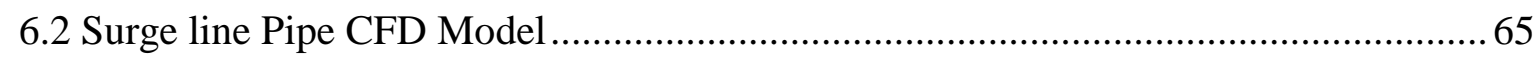

6.2.1 Boundary Conditions 65

6.2.2 Material Properties $\quad 68$

6.3 Preliminary Results from CFD Simulation........................................................... 70

7 Summary and Future Study $\quad 75$

8 References $\quad 77$

Appendices $\quad 80$

Appendix-3A: Supplementary Results for ET-T04 Tensile Test ……………………....... 80

Appendix-3B: Supplementary Results for ET-F06 Fatigue Test........................................... 101

Appendix-3C: Supplementary Results for ET-F41 Fatigue Test...................................... 113

Appendix-3D: Supplementary Results for ET-F38 Fatigue Test ..................................... 127

Appendix-3E: Supplementary Results for ET-F40 Fatigue Test ..................................... 140 


\section{LIST OF FIGURES}

Figure 2. 1 Interpretation of plastic strain energy for a fatigue cycle ......................................... 8

Figure 3. 1 Engineering stress-strain curve estimated from ET-T04 tensile test (in air, $300{ }^{\circ} \mathrm{C}$ ) data for $316 \mathrm{SS}$ base metal. Data point shows the elastic limit stress and strain. ....................... 10

Figure 3. 2 Observed stress during the entire ET-F06 fatigue test. Data points show stress after $1^{\text {st }}$ quarter cycle and the maximum hardening stress and corresponding cycle........................ 11

Figure 3. 3 Observed stress during the entire ET-F41 fatigue test. Data points show stress after $1^{\text {st }}$ quarter cycle and the maximum hardening stress and corresponding cycle........................ 12

Figure 3. 4 Equivalent monotonic stress-strain curves over 50 cycles estimated from ET-F06 fatigue test data and comparison with ET-T04 tensile test data. ......................................... 13

Figure 3. 5 Equivalent monotonic stress-strain curves over 50 cycles estimated from ET-F41 fatigue test data and comparison with ET-T04 tensile test data. .......................................... 13

Figure 3. 6 Time/cycle-dependent elastic modulus estimated from constant-amplitude fatigue tests.

Figure 3. 7 Time/cycle-dependent elastic limit stress estimated from constant-amplitude fatigue tests.

Figure 3. 8 Time/cycle-dependent nonlinear kinematic hardening parameter, $\mathrm{C} 1$ estimated from constant-amplitude fatigue tests................................................................................... 15

Figure 3. 9 Time/cycle-dependent nonlinear kinematic hardening parameter, $\gamma 1$ estimated from

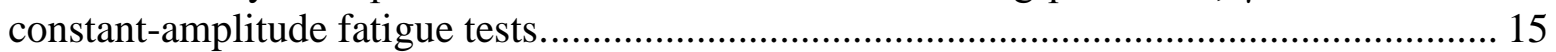

Figure 3. 10 Block loading during variable-amplitude strain-controlled fatigue test (ET-F38). Data points show minimum and maximum amplitudes. ……................................................. 17

Figure 3. 11 Observed stress during the entire ET-F38 fatigue test. .......................................... 18 Figure 3. 12 Observed stress-strain hysteresis behavior during the $1^{\text {st }}$ block (each block comprising 12 cycles) of ET-F38. 18

Figure 3.13 Example comparison of experimental true back stress (as a function of true plastic strain) and predicted back stress (using kinematic hardening equation and estimated parameters: $\mathrm{C} 1$ and $\gamma 1$ pertaining to that block). Black solid line: prediction; Circles: experimental data from 12 fatigue cycles of that block..................................................... 19

Figure 3. 14 Time/block-dependent elastic modulus estimated from variable-amplitude fatigue test (ET-F38).

Figure 3. 15 Time/block-dependent elastic limit stress estimated from variable-amplitude fatigue test (ET-F38).

Figure 3. 16 Time/block-dependent nonlinear kinematic hardening parameter, $\mathrm{C} 1$ estimated from variable-amplitude fatigue test (ET-F38)..................................................................... 20

Figure 3. 17 Time/block-dependent nonlinear kinematic hardening parameter, $\gamma 1$ estimated from variable-amplitude fatigue test (ET-F38)........................................................................... 21

Figure 3. 18 APSE-dependent elastic modulus estimated from variable-amplitude fatigue test (ETF38). The $y$-data corresponding to $\mathrm{x}=0$ is ignored in the semi- $\log \mathrm{x}$ plot. .......................... 21

Figure 3. 19 APSE -dependent elastic limit stress estimated from variable-amplitude fatigue test (ET-F38). The $\mathrm{y}$-data corresponding to $\mathrm{x}=0$ is ignored in the semi-logx plot..................... 22

Figure 3. 20 APSE -dependent nonlinear kinematic hardening parameter, $\mathrm{C} 1$ estimated from variable-amplitude fatigue test (ET-F38). The $y$-data corresponding to $x=0$ is ignored in the

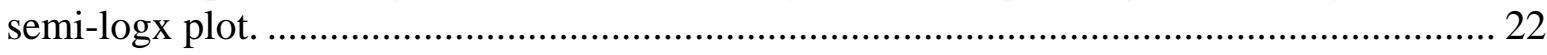


Figure 3. 21 APSE-dependent nonlinear kinematic hardening parameter, $\gamma 1$ estimated from variable-amplitude fatigue test (ET-F38). The $\mathrm{y}$-data corresponding to $\mathrm{x}=0$ is ignored in the semilogx plot. 23

Figure 3. 22 Block loading during random-amplitude strain-controlled fatigue test (ET-F40)... 24

Figure 3. 23 Observed stress during the entire ET-F40 fatigue test. ...................................... 24

Figure 3. 24 Magnified plot of applied strain during ET-F40. .............................................. 25

Figure 3. 25 Magnified plot of observed stress (corresponding to strain shown in Figure 3.24) during ET-F40.

Figure 3. 26 Example hysteresis plot of stress (shown in Figure 3.25) and strain (shown in Figure 3.24) during ET-F40.

Figure 4. 1 Simulated (analytical) vs experimental axial stress history of ET-F41. Predictions are from simulation using time/cycle-dependent parameters (ET-F41) and two sets of timeindependent parameters (tensile test ET-T04 and half-life cycle of ET-F41) ..................... 28

Figure 4. 2 Magnified version of Figure 4.1 showing the ability of evolutionary cyclic plasticity model (time/cycle-dependent prediction) to predict initial hardening behavior of $316 \mathrm{SS}$ under

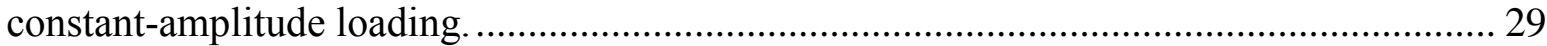

Figure 4. 3 Magnified version of Figure 4.1 showing the ability of evolutionary cyclic plasticity model (time/cycle-dependent prediction) to predict softening behavior of $316 \mathrm{SS}$ under constant-amplitude loading.

Figure 4. 4 Magnified version of Figure 4.1 showing the ability of evolutionary cyclic plasticity model (time/cycle-dependent prediction) to predict the fast stress drop toward the end of the fatigue life of specimen, representing unstable or rapid crack propagation, under constantamplitude loading. 30

Figure 4. 5 Simulated (analytical) vs experimental axial stress history of ET-F06. Predictions are from simulation using time/cycle-dependent parameters from ET-F06 test and two sets of time-independent parameters (tensile test ET-T04 and half-life cycle of ET-F06).............. 30

Figure 4. 6 Simulated (analytical) vs experimental axial stress history of ET-F38. Predictions are from simulation using time/block-dependent parameters (ET-F38) and two sets of timeindependent parameters (tensile test ET-T04 and half-life block of ET-F38). 31

Figure 4. 7 Magnified version of Figure 4.6 showing the ability of evolutionary cyclic plasticity model (time/block-dependent prediction) to predict initial hardening behavior of $316 \mathrm{SS}$ under variable-amplitude loading. 32

Figure 4. 8 Magnified version of Figure 4.6 showing the ability of evolutionary cyclic plasticity model (time/ block -dependent prediction) to predict softening behavior of $316 \mathrm{SS}$ under

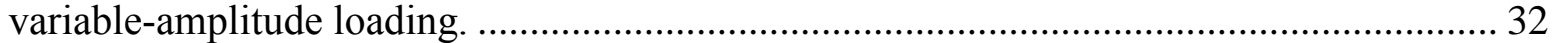

Figure 4. 9 Magnified version of Figure 4.6 showing the ability of evolutionary cyclic plasticity model (time/ block -dependent prediction) to predict the fast stress drop toward the end of the fatigue life of specimen, representing unstable or rapid crack propagation, under variableamplitude loading... 33

Figure 4. 10 Simulated (analytical) vs experimental hysteresis curves of $1^{\text {st }}$ block (with 12 variable-amplitude cycles) of ET-F38. Predictions are from simulation using time/blockdependent parameters and two sets of time-independent parameters (tensile test ET-T04 and half-life block of ET-F38). 33 
Figure 4. 11 Simulated (analytical) vs experimental axial stress history of ET-F41. Prediction is from simulation using APSE-dependent material parameters estimated from variableamplitude test (ET-F38). 35

Figure 4. 12 Simulated (analytical) vs. experimental axial stress history of ET-F06. Prediction is from simulation using APSE-dependent material parameters estimated from variableamplitude test (ET-F38).

Figure 4.13 Simulated (analytical) vs. experimental axial stress history of ET-F38. Prediction is from simulation using APSE-dependent material parameters estimated from variableamplitude test (ET-F38) .................................................................................. 36

Figure 4.14 Simulated (analytical) vs. experimental axial stress history of ET-F40. Predictions are from simulation using APSE-dependent material parameters estimated from variableamplitude test (ET-F38) and fixed parameters estimated from tensile test (ET-T04).......... 37

Figure 4.15 Magnified version of Figure 4.14. Experimental and predicted stress corresponds to applied strain shown in Figure 3.24 .................................................................. 38

Figure 4.16 Simulated (analytical) vs. experimental hysteresis plot of stress shown in Figure 4.15

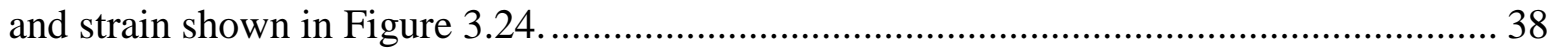

Figure 5. 1 Geometry of fatigue test specimen............................................................... 43

Figure 5.2 Three-dimensional 8-node brick element used for FE simulation of fatigue experiment in ABAQUS.

Figure 5.3 An example simulated stress profile at a given instant during fatigue test simulation.

Figure 5.4 3D-FE simulated (evolutionary cycle plasticity model) vs. experimental axial stress of ET-F41 specimen for first 100 cycles. Predictions are from simulation using time/cycledependent parameters (ET-F41) and two sets of time-independent parameters (tensile test ET-

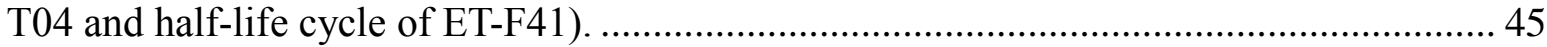

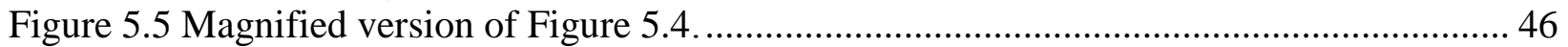

Figure 5.6 3D-FE simulated (evolutionary cycle plasticity model) vs. experimental axial stress of

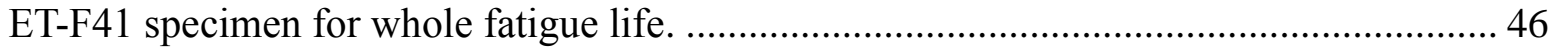

Figure 5.7 Magnified version of Figure 5.6 showing that the 3D-FE results can predict material behavior during initial stress hardening, softening, and stabilized cycles under constantamplitude loading.

Figure 5.8 Magnified version of Figure 5.6 showing that the 3D-FE results can predict material behavior during rapid crack propagation and failure under constant-amplitude loading. .... 47

Figure 5.9 3D-FE simulated (evolutionary cycle plasticity model) vs. experimental axial stress of

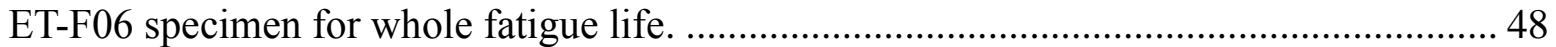

Figure 5.10 3D-FE simulated (evolutionary cycle plasticity model) vs. experimental axial stress of ET-F38 specimen for first 50 blocks. Predictions are from simulation using time/blockdependent parameters (ET-F38) and two sets of time-independent parameters (tensile test ETT04 and half-life block of ET-F38). 49

Figure 5.11 3D-FE simulated (evolutionary cycle plasticity model) vs. experimental axial stress history of ET-F38 specimen for whole fatigue life.

Figure 5.12 Magnified version of Figure 5.11 showing that the 3D-FE results can predict material behavior during initial stress hardening, softening, and stabilized cycles under variableamplitude loading..... 50 
Figure 5.13 Magnified version of Figure 5.11 showing that the 3D-FE results can predict material behavior during rapid crack propagation and failure under variable-amplitude loading...... 51

Figure 5.14 3D-FE simulated (evolutionary cycle plasticity model) vs. experimental axial stress history of ET-F41 specimen for whole fatigue life. Prediction is from simulation using APSEdependent material parameters estimated from variable-amplitude test (ET-F38)............. 52

Figure 5.15 3D-FE simulated (evolutionary cycle plasticity model) vs. experimental axial stress history of ET-F06 specimen for whole fatigue life. Prediction is from simulation using APSEdependent material parameters estimated from variable-amplitude test (ET-F38)............. 53

Figure 5.16 3D-FE simulated (evolutionary cycle plasticity model) vs. experimental axial stress history of ET-F38 specimen for whole fatigue life. Prediction is from simulation using APSEdependent material parameters estimated from variable-amplitude test (ET-F38)............. 54

Figure 5.17 3D-FE simulated (evolutionary cycle plasticity model) vs. experimental axial stress of ET-F40 specimen during $1^{\text {st }}$ block (block period $=4251 \mathrm{~s}$ ) loading. Predictions are from simulation using APSE-dependent material parameters estimated from variable-amplitude test (ET-F38) and fixed parameters estimated from tensile test (ET-T04)........................55

Figure 5.18 Magnified version of Figure 5.17. Experimental and predicted stress correspond to

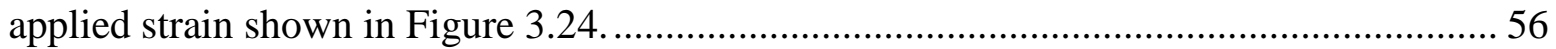

Figure 5.19 3D-FE simulated (evolutionary cycle plasticity model) vs. experimental hysteresis plot of stress shown in Figure 5.18 and strain shown in Figure 3.24 .................................. 56

Figure 5.20 3D-FE simulated (evolutionary cycle plasticity model) vs. experimental axial stress history of ET-F40 specimen for whole fatigue life. Prediction is from simulation using APSEdependent material parameters estimated from variable-amplitude test (ET-F38)............. 57

Figure 5.21 ABAQUS model used for 3D-FE simulation. Nodes indicated are points of interest to investigate the values of APSE and stress in z-direction.

Figure 5.22 APSE as function of fatigue cycles during APSE-based 3D-FE simulation of constantamplitude fatigue test (ET-F41 and ET-F06). APSE values at node-3 and node-6 (see Figure 5.21 for node location in the ABAQUS model) are compared with that at centroid. 61

Figure 5.23 APSE as function of time during APSE-based 3D-FE simulation of random-amplitude fatigue test (ET-F40). APSE values at node-3 and node-6 (see Figure 5.21 for node location in the ABAQUS model) are compared with that at centroid. 62

Figure 5.24 Axial stress as function of fatigue cycles during APSE-based 3D-FE simulation of constant-amplitude fatigue test (ET-F41 and ET-F06). Stress at node-6 (see Figure 5.21 for node location in the ABAQUS model) is compared with that at centroid.

Figure 5.25 Axial stress as function of fatigue cycles during APSE-based 3D-FE simulation of random-amplitude fatigue test (ET-F40). Stress at node-6 (see Figure 5.21 for node location in the ABAQUS model) is compared with that at centroid. 63

Figure 6. 1 ABAQUS model of surge line that connects the hot leg and pressurizer. ............... 66 Figure 6. 2 FE mesh of the SL pipe and the locations of the applied boundary conditions. The location of few representative nodes are highlighted to show some example results discussed later. 66

Figure 6. 3 Applied temperature boundary conditions mimicking the temperature at SL-pressurizer and SL-HL nozzle.

Figure 6. 4 Applied velocity boundary condition along a direction perpendicular to the axis of SL

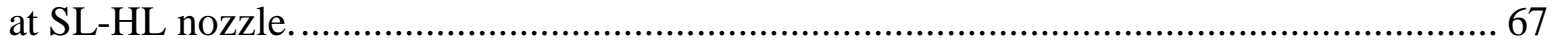

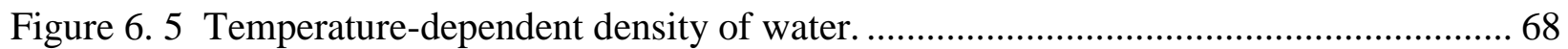




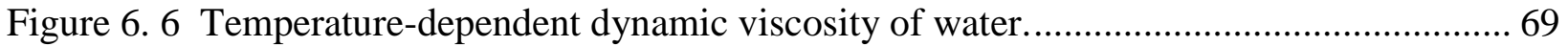

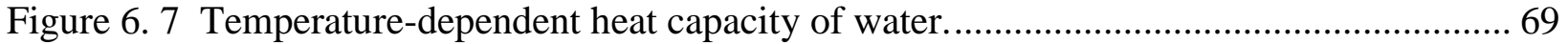

Figure 6.8 Temperature-dependent thermal conductivity of water. ....................................... 70

Figure 6. 9 Simulated temperature history at few representative nodes of SL (as highlighted in

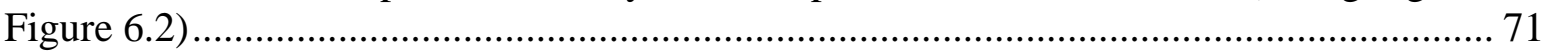

Figure 6. 10 Magnified $\left(\mathrm{t}=30 \times 10^{3} \mathrm{~s}\right.$ to $\left.55 \times 10^{3} \mathrm{~s}\right)$ version of Figure 6.9............................. 71

Figure 6. 11 Temperature contour at a typical instant during heat-up (at $\mathrm{t}=40 \times 10^{3} \mathrm{~s}$ or $11.1 \mathrm{hr}$ ).

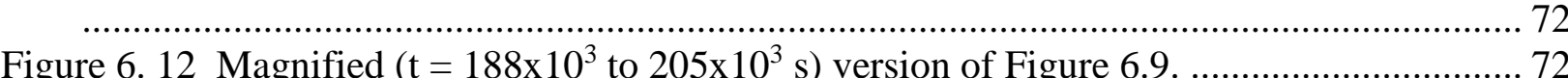

Figure 6. 13 Temperature contour at a typical instant during steady-state power operation $($ at $\mathrm{t}=$

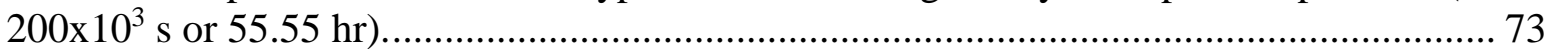

Figure 6. 14 Magnified $\left(\mathrm{t}=328 \times 10^{3} \mathrm{~s}\right.$ to $\left.347.5 \times 10^{3} \mathrm{~s}\right)$ version of Figure $6.9 \ldots \ldots \ldots \ldots \ldots \ldots \ldots . . . \ldots \ldots$

Figure 6. 15 Temperature contour at a typical instant during cool-down (at $\mathrm{t}=340 \times 10^{3} \mathrm{~s}$ or 94.4 hr). 


\section{LIST OF TABLES}

Table 3. 1 Test conditions for 316 SS base metal tensile and fatigue tests. 9

Table 3. 2 Time-independent material parameters estimated from tensile test ET-T04 10

Table 4. 1 Experimentally observed and predicted (analytical modeling) maximum hardening

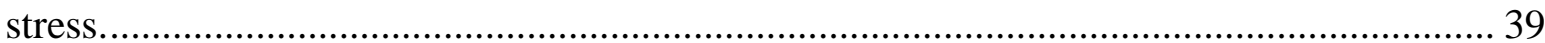

Table 4. 2 Determination of failure criteria for simulation results............................................... 40

Table 4. 3 Experimental and predicted (analytical modeling) fatigue lives. ................................ 41

Table 5. 1 Experimentally observed and predicted (3D-FE modeling) maximum hardening stress.

Table 5. 3 Comparison of APSE-based predicted fatigue lives between analytical modeling and 3D-FE modeling results. 60 


\section{ABBREVIATIONS}

1D One Dimensional

3D Three Dimensional

ANL Argonne National Laboratory

ASME American Society of Mechanical Engineers

APSE Accumulated Plastic Strain Energy

CFD Computational Fluid Dynamics

CL Cold Leg

CORDEL Cooperation in Reactor Design Evaluation and Licensing

FE Finite Element

HL Hot Leg

LAS Low Alloy Steel

LWRS Light Water Reactor Sustainability

PWR Pressurized Water Reactor

SDOs Standards Development Organizations

SL Surge Line

SS Stainless Steel 


\section{ACKNOWLEDGMENTS}

This research was supported through the U.S. Department of Energy's Light Water Reactor Sustainability program under the work package of environmental fatigue study, program manager Dr. Keith Leonard. 
This page intentionally left blank 



\section{Introduction}

The current procedures for fatigue life assessment of nuclear reactor components exposed to thermalmechanical loading cycles and reactor environment are usually based on stress/strain versus life $(\mathrm{S} \sim \mathrm{N})$ curves and/or similar empirical approaches [1-7]. Although these empirical approaches allow engineers to quickly assess the component's design lives, they are not directly based on time-dependent damage evolution and damage accumulation, which can lead to the fatigue failure of metallic components. Furthermore, there remains considerable debate on how to incorporate the effect of the reactor environment while evaluating the fatigue life of reactor components by empirical approaches [8]. Also, as many different codes and standards use different approaches (e.g., based on stress [3-5] or strain range methods $[6,7])$, it is sometimes difficult to decide which method to use. Recently, organizations like the World Nuclear Association's working group Cooperation in Reactor Design Evaluation and Licensing (CORDEL) and the Standards Development Organizations (SDOs) Code Convergence Board have been set up to homogenize the international codes and standards for nuclear power components [9].

By adopting more mechanistic-based approaches for fatigue evaluation, the issues associated with fatigue life evaluation can be greatly reduced. Moreover, with the current availability of advanced computation tools, such as the finite element (FE) method, along with high performance computing, it may be possible to model a component or an overall system more mechanistically than the traditional approach of over dependence on test-based methods. The resulting mechanistic approach will provide engineers with more accurate prediction of fatigue lives of the reactor components as compared with current approaches, which have large uncertainties due to the use of empirical relations.

Nuclear power plant components are subjected to complex stress/strain cycle loading during day-today operations. In some situations, cyclic loading may induce large-amplitude stress reversals, which may exceed the elastic limit due to presence of welds, notches, etc. Under these conditions, the behavior of materials such as 316 stainless steel (SS) [which is primarily used in U.S. reactor primary pipes, such as Pressurized Water Reactor (PWR) surge line, hot leg, and cold leg] may become inelastic and may exhibit related phenomena such as the Bauschinger effect, cyclic hardening/softening, and mean stress relaxation or ratcheting [10]. Thus, the development of advanced models that can address the above-mentioned phenomena and the successful incorporation of those models into a generalized finite element code such as ABAQUS or ANSYS are necessary to ensure more accurate evaluation of the mechanistic-based structural integrity of reactor and other safety-critical components.

Several material models [11-18] have been developed in last few decades to better describe the cyclic plasticity behavior of materials under different loading conditions. Among them, the Chaboche-type [1315] models are found widely used in engineering calculations and included in several commercial FE software, such as ABAQUS and ANSYS. The basis of Chaboche type models is the Armstrong-Frederick non-linear kinematic hardening model [12]. Although various successful simulations were carried out numerically for both rate-independent and rate-dependent plasticity, it was found that the ArmstrongFrederick model over-predicts the cyclic stress relaxation and ratcheting behavior by up to a factor of about ten [17]. Chaboche and Rousselier [15] improved the prediction of ratcheting by superposition of 
multiple Armstrong- Frederick formulas. Although the Chaboche-type model in its original form can accurately describe plasticity under monotonic loading as well as the saturated (or stabilized) cyclic hardening behavior of reactor materials, it may not be accurate for modeling the non-saturation feature of cyclic hardening and/or softening [18]. Even though much improvement of the Chaboche model has been made by other researchers [18-20], none of the existing models in the literature is versatile and robust enough to simulate the cyclic plasticity behavior accurately [21]. Improved understanding of the cyclic plasticity of metal, therefore, is required before a robust plasticity model can be developed for practical applications, such as evaluating the stress-strain state and fatigue life of a reactor component.

In previous work [22-26], we proposed an evolutionary cyclic plasticity model for mechanistic fatigue modeling of key reactor materials. In the present work, we further improved the evolutionary cyclic plasticity model by focusing on model development for $316 \mathrm{SS}$, which is used in U.S. PWRs as primary pipe. This semi-annual progress report presents the work completed on fatigue testing, cyclic plasticity material modeling, 1D-analytical modeling, and 3D-FE modeling of 316 SS laboratory-scale specimens subjected to strain-controlled cyclic loading under constant, variable, and random amplitudes. In this work, we consider material parameters as functions of time and/or accumulated plastic strain energy (APSE). The APSE approach is in addition to our earlier work [22-26] to attain more robust cyclic plasticity modeling under real-life random/arbitrary loading conditions. The related time-based and APSE-based parameters for 316 SS base metal obtained through various strain control tests are presented. The resulting evolutionary cyclic plasticity model was validated through analytical modeling of various fatigue experiments, including constant, variable, and random amplitude. We also improved our evolutionary cyclic plasticity model (which is a first of its kind) so that it can be incorporated into commercially available FE code such as ABAQUS to perform 3D-FE simulation of the fatigue experiments. Furthermore, some preliminary CFD model results related to evaluation of temperature boundary condition and stratification of surge line pipes are presented. 


\section{Theoretical Background on Evolutionary Cyclic Plasticity: APSE-Based 1D-Analytical and 3D-FE Modeling}

An evolutionary cyclic plasticity model was developed based on Chaboche [13] or ArmstrongFrederick [12] type analytical expressions for nonlinear kinematic hardening. The details of the material model development are discussed in our previous work [22, 24]. However, for the completeness of this report the evolutionary cyclic plasticity model and related constitutive relations are presented here briefly. Note that the constitutive relations are in more generalized form. Previously [22, 24], we presented a cycle-by-cycle technique to estimate time/cycle-dependent parameters from experimental data from a constant-amplitude uniaxial fatigue test. In later work [26], we presented a more generalized estimation scheme (refer to "method-2" in the reference), which can be used for estimating time/cycle-dependent or time/block-dependent material parameters from constant- or variable-amplitude test data, respectively. We briefly present the generalized estimation technique here. In addition, since material behavior under random-amplitude fatigue loading cannot be modeled by using earlier developed time-dependent material parameters, we discuss the possibility of using an APSE-dependent material model. To accommodate the APSE-based approach in a more generalized framework (than the earlier presented $[22,24,26]$ time/cycle based model), the constitutive relations are symbolically expressed with respect to a generalized field variable $v$, which can be a function of time or fatigue cycle/block or any other physical state (e.g., APSE). The details of the constitutive relations and other analytical expressions are given in the following subsections. Furthermore, at the end of this section, the implementation of our evolutionary cyclic plasticity model into a commercial FE software, ABAQUS, is also discussed.

\subsection{Constitutive Relations for Evolutionary Cyclic Plasticity Model}

We consider a rate-independent elastic-plastic material. We assume the strain is small and can be additively decomposed into an elastic part obeying Hooke's law and a plastic part governed by the associated flow rule based on Von-Mises yield criteria $(\mathrm{f}=0)$. At any instant, the yield function $f_{v}$, corresponding to the evolutionary cyclic plasticity model, can be expressed as,

$$
f_{v}(\boldsymbol{\sigma}, \boldsymbol{\alpha})=\sqrt{\frac{3}{2}(\boldsymbol{S}-\boldsymbol{\alpha}):(\boldsymbol{S}-\boldsymbol{\alpha})}-\sigma_{v}^{y}=0
$$

where $\boldsymbol{\sigma}$ is the stress vector at that instant, $\boldsymbol{\alpha}$ is the corresponding back stress vector, and $\sigma_{v}^{y}$ is the yield stress. Note that the subscript $v$ is used to demonstrate that the yield stress is not constant as it is in the case of conventional cyclic plasticity model, rather it is a variable that can be a function of time or fatigue cycle/block or any other physical state (e.g., APSE). In addition, $\boldsymbol{S}$ represents the deviatoric stress portion of $\boldsymbol{\sigma}$. The associated flow rule can be expressed as:

$$
d \varepsilon=d P \frac{\partial f_{v}}{\partial \sigma}
$$

where $d P$ is the effective plastic strain increment. According to the additive rule the total strain can be expressed as:

$$
\boldsymbol{\varepsilon}=\boldsymbol{\varepsilon}^{e l}+\varepsilon^{p l}
$$

with Hooke's law for elastic strain, 


$$
\boldsymbol{\sigma}=\boldsymbol{C}_{v} \boldsymbol{\varepsilon}^{e l}=\boldsymbol{C}_{v}\left(\boldsymbol{\varepsilon}-\boldsymbol{\varepsilon}^{p l}\right)
$$

where $\boldsymbol{\varepsilon}^{e l}$ and $\boldsymbol{\varepsilon}^{p l}$ are the elastic and plastic portion of the strain $(\boldsymbol{\varepsilon})$ at that instant and $\boldsymbol{C}_{\boldsymbol{v}}$ is the variable elastic stiffness matrix.

The evolutionary cyclic plasticity model based on Chaboche-type nonlinear kinematic hardening can be expressed as,

$$
d \boldsymbol{\alpha}=\frac{2}{3} C 1_{v} d \boldsymbol{\varepsilon}-\gamma 1_{v} \boldsymbol{\alpha} d P
$$

where $C 1_{v}$ and $\gamma 1_{v}$ are material parameters. The parameter $C 1_{v}$ is a proportional constant that gives a linear relation between the increment in the back stress, $d \boldsymbol{\alpha}$, and the increment in the plastic strain, $d \boldsymbol{\varepsilon}^{p l}$, while $\gamma 1_{v}$ describes the rate at which the back stress decreases with the increase in accumulated effective plastic strain, $d P$.

For incremental plasticity, the plastic strain increment, $d \boldsymbol{\varepsilon}^{p l}$, associated with the nonlinear kinematic hardening rule, given in Eq. (2.5), can be expressed as

$$
d \boldsymbol{\varepsilon}^{p l}=\left(\frac{\frac{\partial f_{v}}{\partial \sigma} \cdot C_{v} d \varepsilon}{\left(\frac{\partial f_{v}}{\partial \sigma}\right) \cdot c_{v}\left(\frac{\partial f_{v}}{\partial \sigma}\right)+\gamma 1_{v}\left(\frac{\partial f_{v}}{\partial \alpha}\right) \cdot \boldsymbol{\alpha}-\frac{2}{3} C 1_{v}\left(\frac{\partial f_{v}}{\partial \alpha}\right) \cdot\left(\frac{\partial f_{v}}{\partial \alpha}\right)}\right) \frac{\partial f_{v}}{\partial \sigma}
$$

The above expression for the incremental plastic strain can be used for developing a cyclic-plasticity finite element or analytical model. The associated parameters include the variable elastic modulus ( $E_{v}$, used for estimating the stiffness matrix $\left.\boldsymbol{C}_{v}\right)$, kinematic hardening parameters $\left(C 1_{v}\right.$ and $\left.\gamma 1_{v}\right)$, and yield stress $\left(\sigma_{v}^{y}\right.$, used for estimating the yield function $f_{v}$ ).

\subsection{Time-Based Estimation of Time-Dependent Material Parameters}

Time-dependent material parameters are estimated from either a constant- or a variable-amplitude fatigue test. Stress-strain data from all the cycles within a block are used together to estimate time/blockdependent (i.e., block-by-block) material parameters from variable-amplitude tests. In addition, stressstrain data from each cycle are used to estimate time/cycle-dependent material parameters from constantamplitude test. Here we briefly present our block-by-block parameter estimation technique, which can also be used to estimate cycle-by-cycle parameter estimation. To estimate block-dependent material parameters, all the cyclic stress-strain curves in a block are first converted into equivalent monotonic stress-strain curves. Based on an assumed elastic limit strain, the material parameters are then determined for all the blocks by using the Gauss-Newton optimization scheme. The steps for estimating the material parameters for block $i$ are as follows:

Step 1: Estimate the kinematic hardening stress or back stress $\left(\alpha_{i, j, k}\right)$ and corresponding accumulated intra-cycle plastic strain $\left(\bar{p}_{i, j, k}\right)$ for $\mathrm{k}$ instances $\left(\mathrm{k}=1,2, \ldots \mathrm{m}_{\mathrm{j}}\right)$ using the expressions:

$$
\alpha_{i, j, k}=\sigma_{i, j, k}-\sigma_{i}^{y}
$$




$$
\bar{p}_{i, j, k}=\varepsilon_{i, j, k}-\frac{\sigma_{i}^{y}}{E_{i}}
$$

where subscript $j$ represents the $\mathrm{j}^{\text {th }}(\mathrm{i}=1,2, . .2 \mathrm{n})$ up/down cycle of the $\mathrm{i}^{\text {th }}$ block, superscript $k$ represents the $\mathrm{k}^{\text {th }}$ data point in the shifted and scaled $\mathrm{j}^{\text {th }}(\mathrm{i}=1,2, . .2 \mathrm{n})$ up/down cycle of the $\mathrm{i}^{\text {th }}$ block, and $\sigma_{i}^{y}$ and $E_{i}$ are the average yield stress and average elastic modulus in the $\mathrm{i}^{\text {th }}$ block, respectively. Note that there are $\mathrm{n}$ fatigue cycles in each block, i.e., $\mathrm{n}$ upward cycles and $\mathrm{n}$ downward cycles, which make the total number of monotonic cycles in each block 2 n.

Step 2: Assume initial values of nonlinear kinematic hardening parameters: $\boldsymbol{L}=\left[\begin{array}{ll}C 1_{i} & \gamma 1_{i}\end{array}\right]^{T}$

Step 3: Estimate the residual function vector

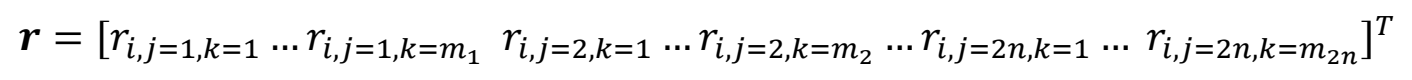

with the $\mathrm{k}^{\text {th }}$ instance residual in the $\mathrm{j}^{\text {th }}$ cycle of the $\mathrm{i}^{\text {th }}$ block as

$$
r_{i, j, k}=\frac{C 1_{i}}{\gamma 1_{i}}\left[1-\exp \left(\left(-\gamma 1_{i}(p) \bar{p}_{i, j, k}\right)\right)\right]-\alpha_{i, j, k}
$$

Step 4: Estimate the Jacobian matrix $J$ as follows:

$$
J=\left[\begin{array}{cc}
\frac{\partial r_{i, j=1, k=1}}{\partial C 1_{i}} & \frac{\partial r_{i, j=1, k=1}}{\partial \gamma 1_{i}} \\
\vdots & \vdots \\
\frac{\partial r_{i, j=1, k=m_{1}}}{\partial C 1_{i}} & \frac{\partial r_{i, j=1, k=m_{1}}}{\partial \gamma 1_{i}} \\
\frac{\partial r_{i, j=2, k=1}}{\partial C 1_{i}} & \frac{\partial r_{i, j=2, k=1}}{\partial \gamma 1_{i}} \\
\vdots & \vdots \\
\frac{\partial r_{i, j=2, k=m_{2}}}{\partial C 1_{i}} & \frac{\partial r_{i, j=2, k=m_{2}}}{\partial \gamma 1_{i}} \\
\vdots & \vdots \\
\frac{\partial r_{i, j=2 n, k=1}}{\partial C 1_{i}} & \frac{\partial r_{i, j=2 n, k=1}}{\partial \gamma 1_{i}} \\
\vdots & \vdots \\
\frac{\partial r_{i, j=2 n, k=m_{2 n}}}{\partial C 1_{i}} & \frac{\partial r_{i, j=2 n, k=m_{2 n}}}{\partial \gamma 1_{i}}
\end{array}\right]
$$

In Eq. 2.11, the $\mathrm{k}^{\text {th }}$ instance expression for the partial derivatives is given below:

$$
\frac{\partial r_{i, j, k}}{\partial C 1_{i}}=\frac{1}{\gamma 1_{i}}\left[1-\exp \left(\left(-\gamma 1_{i} \bar{p}_{i, j, k}\right)\right)\right]
$$

and

$$
\frac{\partial r_{i, j, k}}{\partial \gamma 1_{i}}=\frac{-C 1_{i}}{\left(\gamma 1_{i}\right)^{2}}\left[1-\exp \left(-\gamma 1_{i} \bar{p}_{i, j, k}\right)\right]+\frac{C 1_{i} \bar{p}_{i, j, k}}{\left(\gamma 1_{i}\right)} \exp \left(-\gamma 1_{i} \bar{p}_{i, j, k}\right)
$$


Step 5: Estimate the incremental change in parameters:

$$
\Delta \boldsymbol{L}=\left[\Delta C 1_{i} \Delta \gamma 1_{i}\right]^{T}=-\left[\left(\boldsymbol{J}^{T} \boldsymbol{J}\right)^{-1} \boldsymbol{J}^{T}\right] \boldsymbol{r}
$$

Step 6: Update parameters as:

$$
\boldsymbol{L}=\left[\begin{array}{ll}
C 1_{i} & \gamma 1_{i}
\end{array}\right]^{T}=\boldsymbol{L}+\Delta \boldsymbol{L}
$$

Step 7: Repeat step 3 to step 6 unless the $\mathrm{L}_{2}$ norm of the incremental parameters $\Delta \boldsymbol{L}$ is less than a tolerance value, i.e.,

$$
\|\Delta \boldsymbol{L}\|_{2} \leq t_{t o l}
$$

In Eqs. 2.7 to 2.16, the subscripts i, j, and k respectively represent block number, cycle number in block $\mathrm{i}$, and data number in cycle $\mathrm{j}$. This is the convention used for estimating time/block-dependent material parameters from variable-amplitude fatigue tests, while for estimating time/cycle-dependent material parameters from constant-amplitude fatigue tests, $\mathrm{i}$ and $\mathrm{j}$ are considered to be the same.

\subsection{Modeling Fatigue Behavior using Evolutionary Cyclic Plasticity Model}

The major aim of our research is to develop an FE modeling framework based on the proposed evolutionary cyclic plasticity model for estimating the lives of nuclear reactor components subjected to random cyclic loading. The cyclic plasticity model must be validated for laboratory experimental data through analytical modeling and 3D-FE modeling of the specimen before it can be implemented on component-level 3D-FE modeling. In this work, we modeled various fatigue experiment cases using the evolutionary cyclic plasticity model. The two modeling schemes used are discussed in the following subsections.

\subsubsection{Time-Based Modeling}

Material behavior under constant- or variable-amplitude fatigue loading can be predicted from the constitutive relations (Eqs. 2.1 to 2.6) for the evolutionary cyclic plasticity model and the time-dependent material parameters estimated via the technique described in Section 2.2. For a constant-amplitude fatigue loading, time/cycle-dependent (i.e., cycle-by-cycle) parameters are used while time/block-dependent (i.e., block-by-block) parameters are used to model material behavior under variable-amplitude block loading. For analytical modeling of the evolutionary cyclic plasticity model, we adopted the radial return method proposed by Wilkins [27]. Using this method, the stress increment for a given strain increment is computed. Results from time-based analytical modeling of the fatigue tests are presented in Section 4. 


\subsubsection{APSE-Based Modeling}

As discussed in Section 2.3.1, time-dependent material parameters are used to model material fatigue behavior under constant- or variable-amplitude loading. However, time-dependent material parameters cannot be used when the test material is subjected to random-amplitude loading. In this case, material parameters must be expressed as functions of a non-time physical/field variable that changes as fatigue progresses. Here, we consider APSE as the physical variable that changes as material fatigue progresses.

As the fatigue process involves a dissipation of strain energy in each cycle of loading due to the irreversibility of the micro-plastic deformation, fatigue life can be related to the strain energy [28]. According to Lin et al. [29], the total dissipated energy $\left(\mathrm{W}_{\mathrm{f}}\right)$ during a fatigue cycle can be divided into

$$
\mathrm{W}_{\mathrm{f}}=\mathrm{W}_{\mathrm{p}}+\mathrm{Q}+\mathrm{E}_{\mathrm{o}}+\mathrm{E}_{\mathrm{k}}
$$

where $\mathrm{W}_{\mathrm{p}}$ is the plastic energy; $\mathrm{Q}$ is the thermal energy, $\mathrm{E}_{\mathrm{k}}$ is the kinematic energy, and $\mathrm{E}_{\mathrm{o}}$ is other kind of energy. The kinetic energy and the other kinds of energy tend to zero [30]. Moreover, if the tests are done at constant temperature, without the carrying away of heat but with low cycle fatigue loading, the thermal energy $\mathrm{Q}$ is zero [30]. Thus, the total energy dissipation is

$$
\mathrm{W}_{\mathrm{f}}=\mathrm{W}_{\mathrm{p}}
$$

The plastic strain energy of a fatigue cycle is defined by the area bounded by the hysteresis loop in the cyclic stress-strain curve [31], as illustrated in Figure 2.1. The plastic strain energy can be calculated by simply integrating the stress-strain plot. We used the trapezoidal rule of numerical integration to calculate the area. Plastic strain energy of the stabilized cycle or plastic strain energy density (i.e., APSE divided by number of fatigue cycles) has been used by many researchers [28-35] for damage evaluation of material under low cycle fatigue loading. By considering similar maximum strain amplitude applied during all the fatigue tests, where all the tests were done at same strain rate, we assume the APSE value at the failure of a specimen to be close for all the test cases. Therefore, the APSE was used as a physical variable for fatigue modeling in the random-amplitude test. The material parameters estimated from the variableamplitude test are used as function of APSE for APSE-based fatigue modeling 


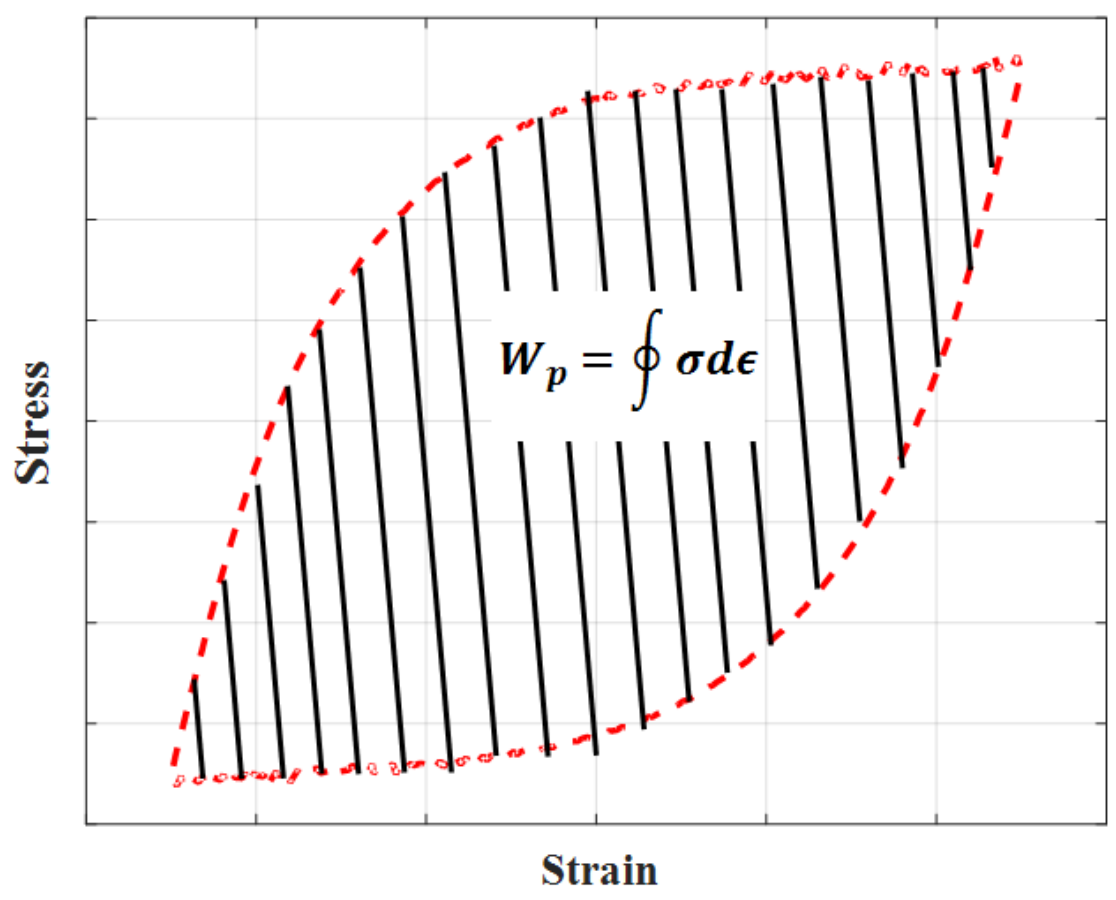

Figure 2. 1 Interpretation of plastic strain energy for a fatigue cycle.

\subsection{FE Implementation of Evolutionary Cyclic Plasticity Model}

The cyclic plasticity model was incorporated into the developed FE code for Chaboche-type models in the ABAQUS/Standard environment. As ABAQUS uses the backward Euler method [36], it provides unconditional stability for integration of rate equations. However, ABAQUS does not allow the use of time/cycle-dependent material properties. A user subroutine, called USDFLD (written in Fortran), was developed to enable the use of time- or APSE-dependent material properties in the implementation of the evolutionary cyclic plasticity model into ABAQUS. The time- and APSE-dependent material properties such as elastic modulus, yield stress, and kinematic hardening parameters are provided in the ABAQUS input file in tabular form, where the cycle/block numbers or APSE values (calculated at the end of each block from variable-amplitude fatigue test results) are assigned under a user-defined field variable. At the start of each integration, the USDFLD accesses the current time, from which it calculates current cycle/block number in the case of time-based modeling. In the case of APSE-based simulation, it accesses a user-defined state variable, where the APSE from the previous time step has been stored. The USDFLD then accesses the corresponding material properties from the table provided in the input file. If properties are not provided for a value of the user-defined field variable, ABAQUS uses interpolation to calculate the material properties. 


\section{Results from Strain Control Tensile/Fatigue Test and Material Parameter Estimation Models of 316 SS}

Strain-controlled uniaxial tensile/fatigue experiments were conducted on 316 SS base metal using small hourglass specimens. The experimental data were used to estimate material parameters and to validate the evolutionary cyclic plasticity model through analytical and 3D-FE modeling of the fatigue experiments. Constant, variable, and random strain amplitudes were employed during the fatigue experiments. All the tests were performed in air at $300{ }^{\circ} \mathrm{C}$ using a hydraulic-controlled MTS test frame. The details of the test setup can be found in a previous Argonne report [24]. Experimental data from a previously [37] conducted strain controlled tensile test (ET-T04) in air at $300{ }^{\circ} \mathrm{C}$ were also used in this work. A constant strain rate of $0.1 \% / \mathrm{s}$ was used during both the tensile and fatigue experiments. The test conditions along with the test IDs are presented in Table 3.1. It should be noted here that the experimental results from the constant-amplitude fatigue test ET-F06 were published in our earlier report [22] but are presented here since the resulting test data are used for analytical FE model validation, which are discussed in the later part of this report.

Table 3. 1 Test conditions for 316 SS base metal tensile and fatigue tests.

\begin{tabular}{|c|l|}
\hline Test ID & \multicolumn{1}{|c|}{ Test Condition } \\
\hline ET-T04 & In air, $300{ }^{\circ} \mathrm{C}$, strain control, tensile test [37] \\
\hline ET-F06 & In air, $300{ }^{\circ} \mathrm{C}$, strain control, constant amplitude $=0.5 \%$, fatigue test [22] \\
\hline ET-F38 & In air, $300{ }^{\circ} \mathrm{C}$, strain control, variable amplitude $=0.05 \%$ to $0.55 \%$, fatigue test \\
\hline ET-F40 & $\begin{array}{l}\text { In air, } 300 ~ \\
\text { fatigue test }\end{array}$ \\
\hline EN-F41 strain control, maximum strain $=0.55 \%$, minimum strain $=-0.55 \%$, \\
In air, $300{ }^{\circ} \mathrm{C}$, strain control, constant amplitude $=0.5 \%$, fatigue test \\
\hline
\end{tabular}

\subsection{Tensile Test and Associated Time/Cycle Independent Elastic-Plastic Material Model Results}

Figure 3.1 depicts the engineering stress-strain plot (up to $2 \%$ strain) estimated from tensile test ETT04. The tensile test data were further analyzed to evaluate fixed material parameters such as tensile modulus, elastic stress limit, and the non-linear kinematic hardening parameters (C1 and $\gamma 1)$. These fixed material parameters were then used to model all the fatigue test cases discussed in this report. The predicted (using analytical and 3D-FE models) stress profiles using tensile test based fixed parameters were then compared with the experimental data. The predicted stress profiles were also compared with those predicted using time/cycle-dependent material properties. The fixed material parameters estimated from ET-T04 test are provided in Table 3.2. 


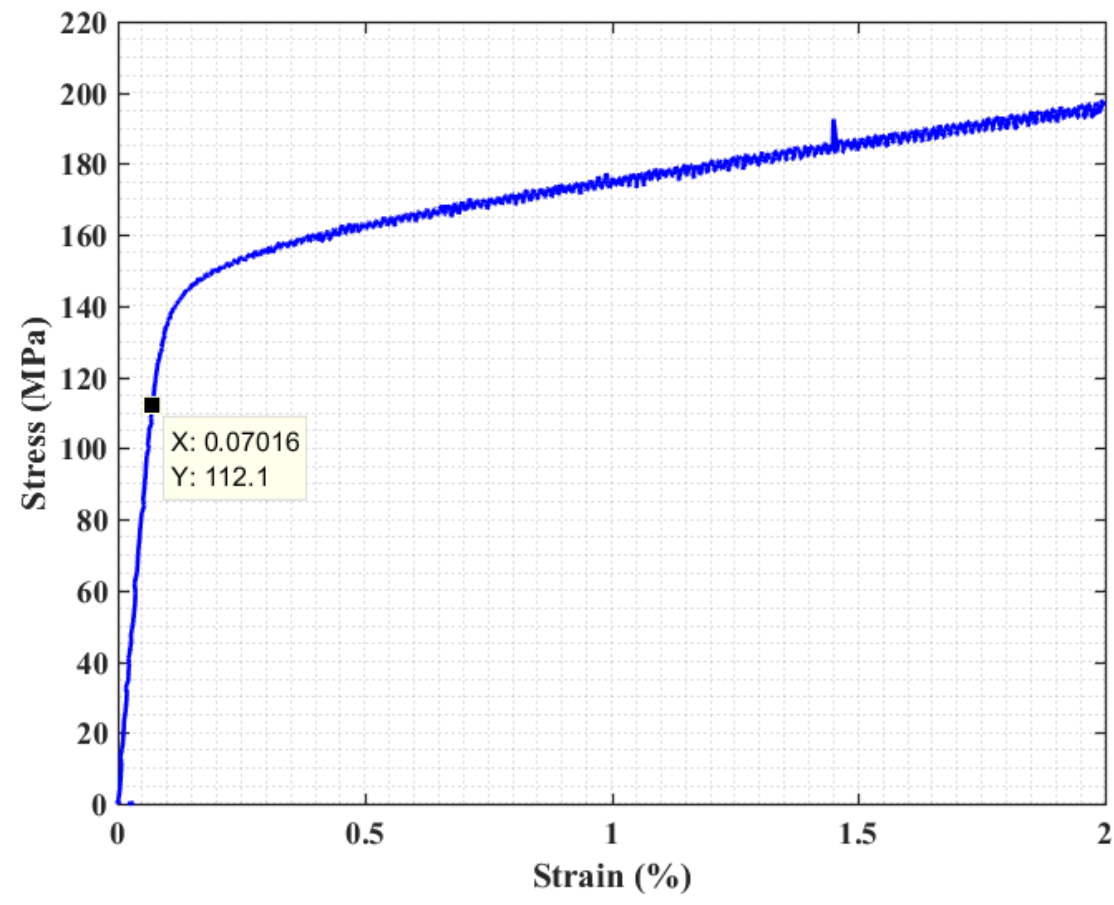

Figure 3. 1 Engineering stress-strain curve estimated from ET-T04 tensile test (in air, $300{ }^{\circ} \mathrm{C}$ ) data for 316 SS base metal. Data point shows the elastic limit stress and strain.

Table 3. 2 Time-independent material parameters estimated from tensile test ET-T04

\begin{tabular}{|c|c|c|c|}
\hline \multirow{2}{*}{ Elastic modulus (GPa) } & \multirow{2}{*}{$\begin{array}{c}\text { Elastic limit stress used in } \\
\text { models as yield stress (MPa) }\end{array}$} & \multicolumn{2}{|c|}{ Nonlinear kinematic hardening parameters } \\
\cline { 3 - 4 } & 112 & $\mathrm{C} 1(\mathrm{MPa})$ & $\gamma 1$ \\
\hline 167 & & 21488 & 279 \\
\hline
\end{tabular}

\subsection{Constant-Amplitude Fatigue Test and Associated Cyclic Elastic-Plastic Material Model Results}

Two constant-amplitude fatigue tests (ET-F06 and ET-F41) were performed under the same conditions. As mentioned in Table 3.1, the applied strain amplitude was $0.5 \%$ during the constantamplitude fatigue tests. The time histories of the observed stress from fatigue experiments ET-F06 and ET-F41 are shown in Figures 3.2 and 3.3, respectively. The curves show that the material exhibits initial cyclic hardening followed by cyclic softening. The maximum hardening stress was $249.8 \mathrm{MPa}$ for ETF06 and 244.5 MPa for ET-F41. The difference (5.3 MPa) in the maximum hardening stresses can be explained by considering the difference in the stresses after the $1^{\text {st }}$ quarter cycle (162.6 MPa for ET-F06 
and 158.7 MPa for ET-F41; difference: 3.9 MPa), which represents the initial (i.e., tensile) behavior of the specimen before application of any fatigue/cycle loading. In both cases, the maximum hardening stress was observed at the $53^{\text {rd }}$ cycle. However, there is a big difference in the fatigue lives for the two specimens. The fatigue life was 4202 cycles for the ET-F06 specimen and 6918 cycles for the ET-F41 specimen. The criteria for fatigue life estimation are discussed in Section 7. Considering the intrinsic nature of fatigue failure in metallic materials, this difference in fatigue lives under the same loading condition is not unexpected. It is due to the usual scatter in material microstructure. Thus, many repeat tests are needed to estimate average material properties along with their statistical variations, which need to be included in evaluating the lives of the components.

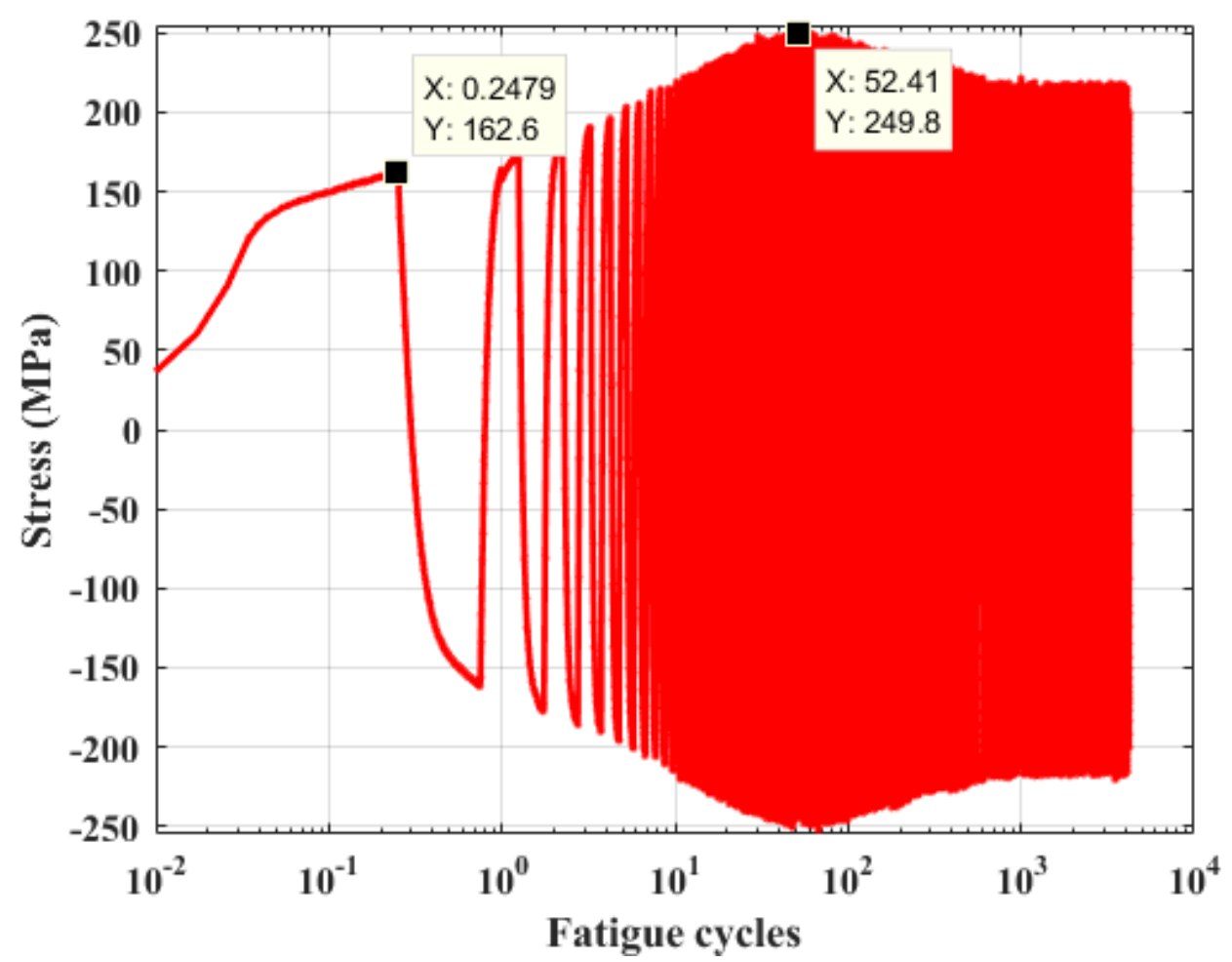

Figure 3. 2 Observed stress during the entire ET-F06 fatigue test. Data points show stress after $1^{\text {st }}$ quarter cycle and the maximum hardening stress and corresponding cycle. 


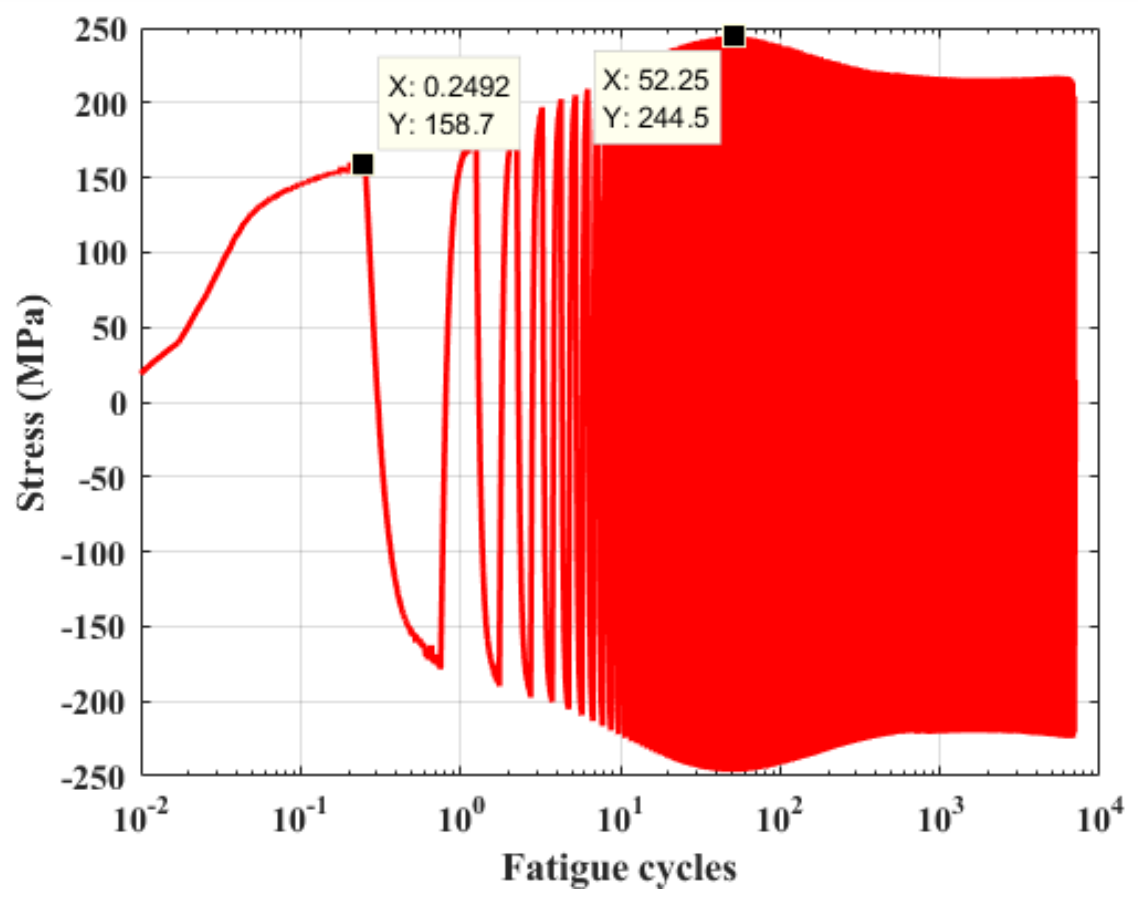

Figure 3. 3 Observed stress during the entire ET-F41 fatigue test. Data points show stress after $1^{\text {st }}$ quarter cycle and the maximum hardening stress and corresponding cycle.

Time/cycle-dependent material properties were estimated from the two constant-amplitude fatigue tests (ET-F06 and ET-F41). To evaluate the material properties, equivalent monotonic curves estimated from the cyclic stress-strain data (acquired from the ET-F06, ET-F41 fatigue tests) were used (for details refer to our earlier work [24]). Figures 3.4 and 3.5 depict the equivalent-monotonic curves estimated from the first 50 fatigue cycles of ET-F06 and ET-F41 test, respectively. The stress-strain curve from tensile test T-04 is also shown in the figures. It can be seen from the figure that the stress at $0.5 \%$ strain increases from $\sim 160 \mathrm{MPa}$ to $250 \mathrm{MPa}$, which again verifies a significant amount of stress hardening/softening during the fatigue experiment and hence the importance of the evolutionary cyclic plasticity model for more accurate fatigue evaluation of $316 \mathrm{SS}$. The equivalent-monotonic stress-strain curves were further analyzed to estimate the time/cycle-dependent material parameters, such as elastic modulus, elastic limit stress (or yield stress), and kinematic hardening parameters (C1and $\gamma 1$ ). Section 2 summarizes the time/cycle-dependent parameter estimation technique, which was first reported by our research group in an earlier report [24].

The estimated parameters are shown in Figures 3.6 to 3.9. Figures 3.6 and 3.7 show the time/cycle variation of elastic modulus and elastic limit stress, respectively, whereas Figures 3.8 and 3.9 show the nonlinear kinematic hardening parameters $\mathrm{C} 1$ and $\gamma 1$, respectively. As seen in the figures, the material parameters vary significantly (particularly the nonlinear kinematic hardening parameters in this case) over the fatigue cycles, in contrast to the tensile-test-based fixed parameters (refer to Table 3.2). The variations of the cycle-by-cycle material parameters are similar for both test cases (ET-F06 and ET-F41), where the differences that do exist are due to the variations in stress between the two tests, as discussed before. 


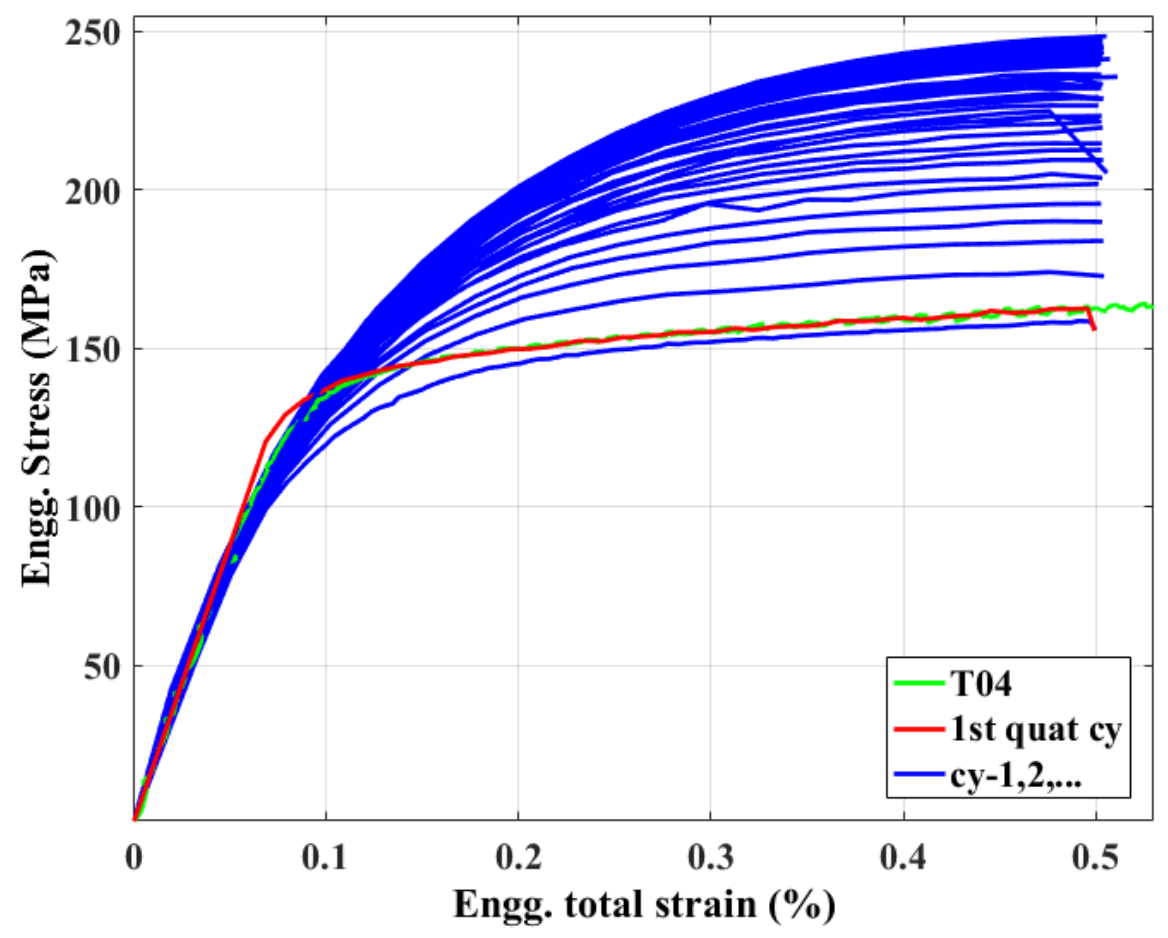

Figure 3. 4 Equivalent monotonic stress-strain curves over 50 cycles estimated from ET-F06 fatigue test data and comparison with ET-T04 tensile test data.

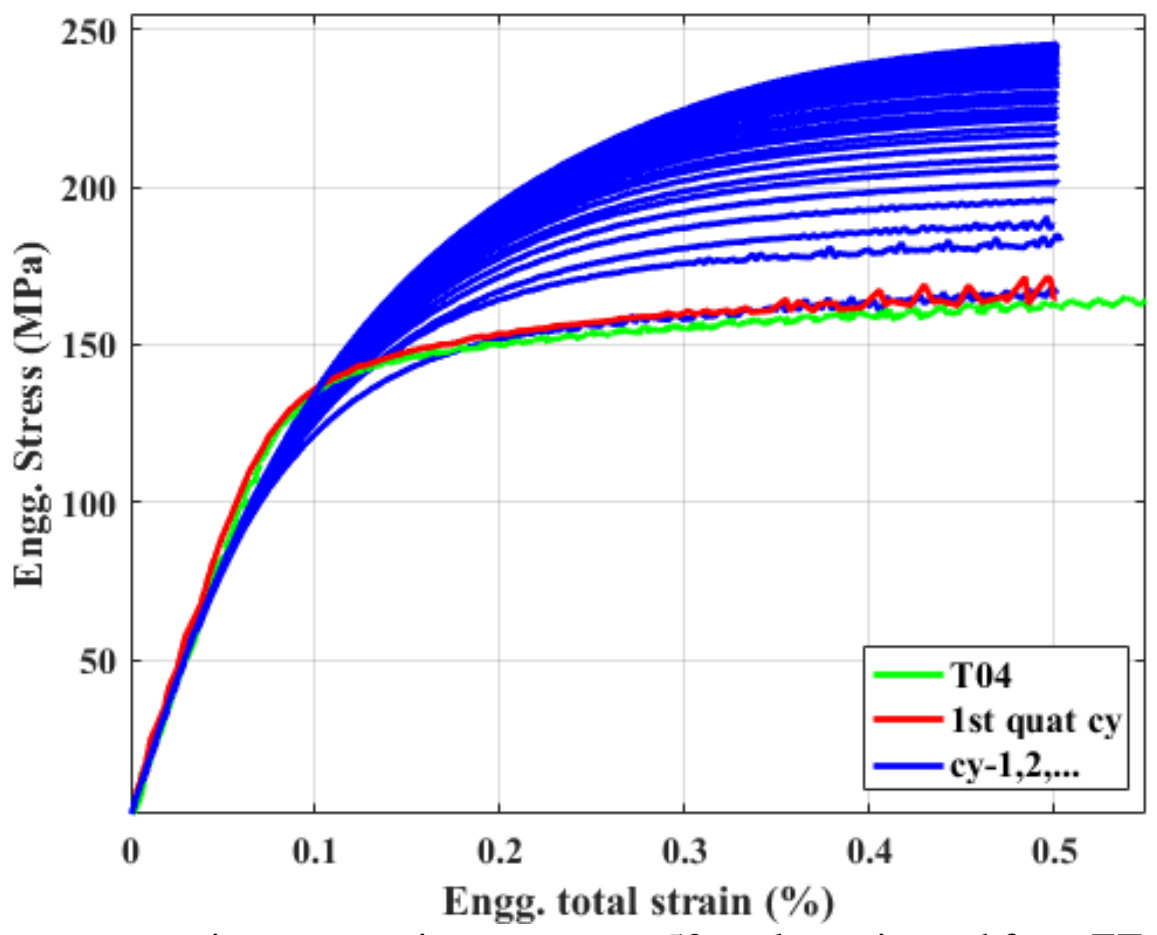

Figure 3. 5 Equivalent monotonic stress-strain curves over 50 cycles estimated from ET-F41 fatigue test data and comparison with ET-T04 tensile test data. 


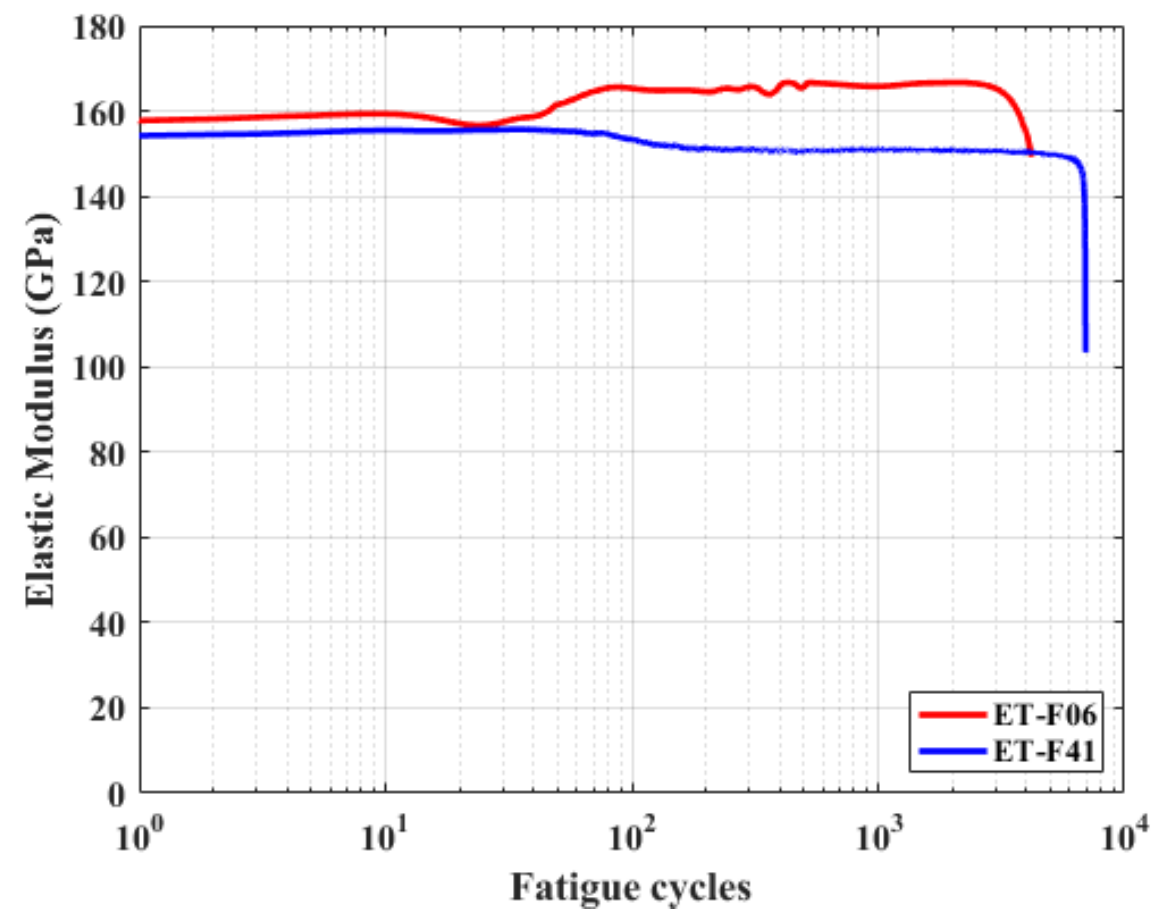

Figure 3. 6 Time/cycle-dependent elastic modulus estimated from constant-amplitude fatigue tests.

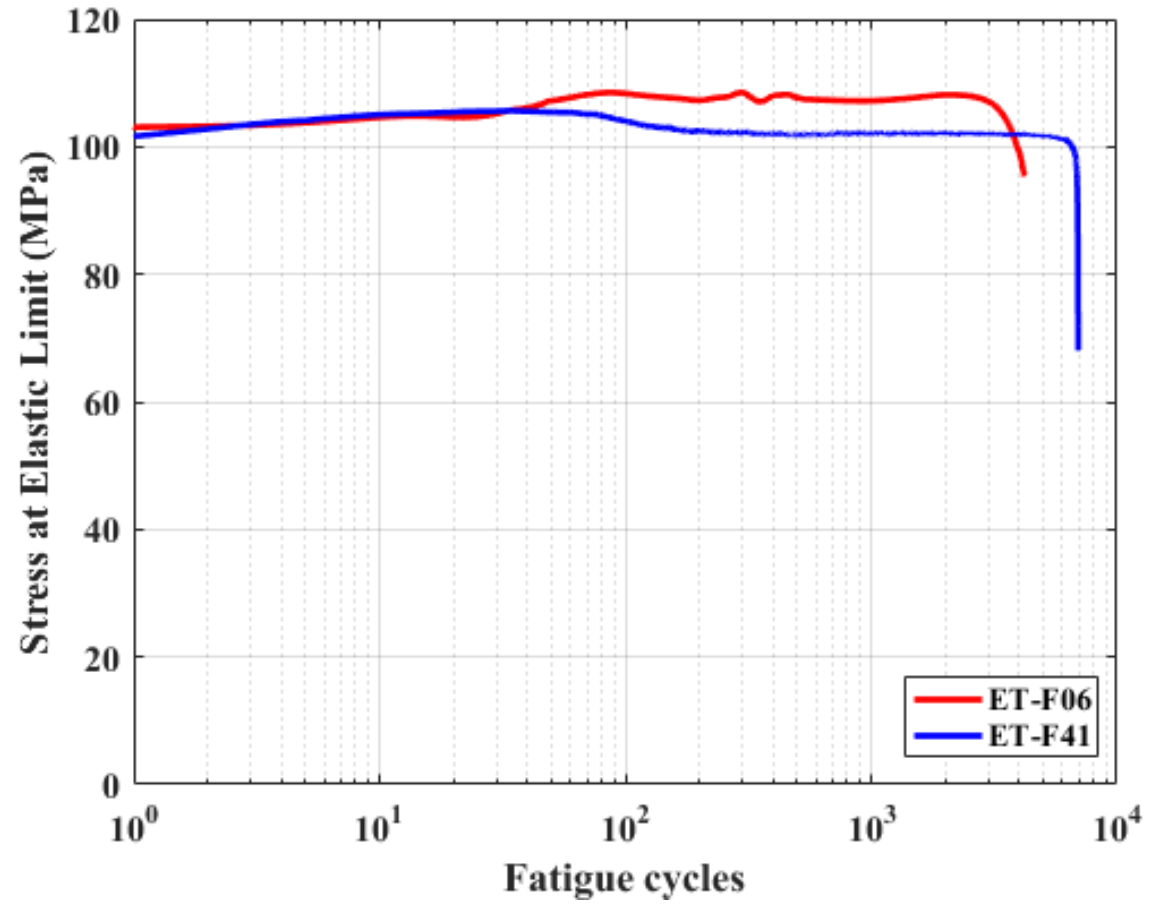

Figure 3. 7 Time/cycle-dependent elastic limit stress estimated from constant-amplitude fatigue tests. 


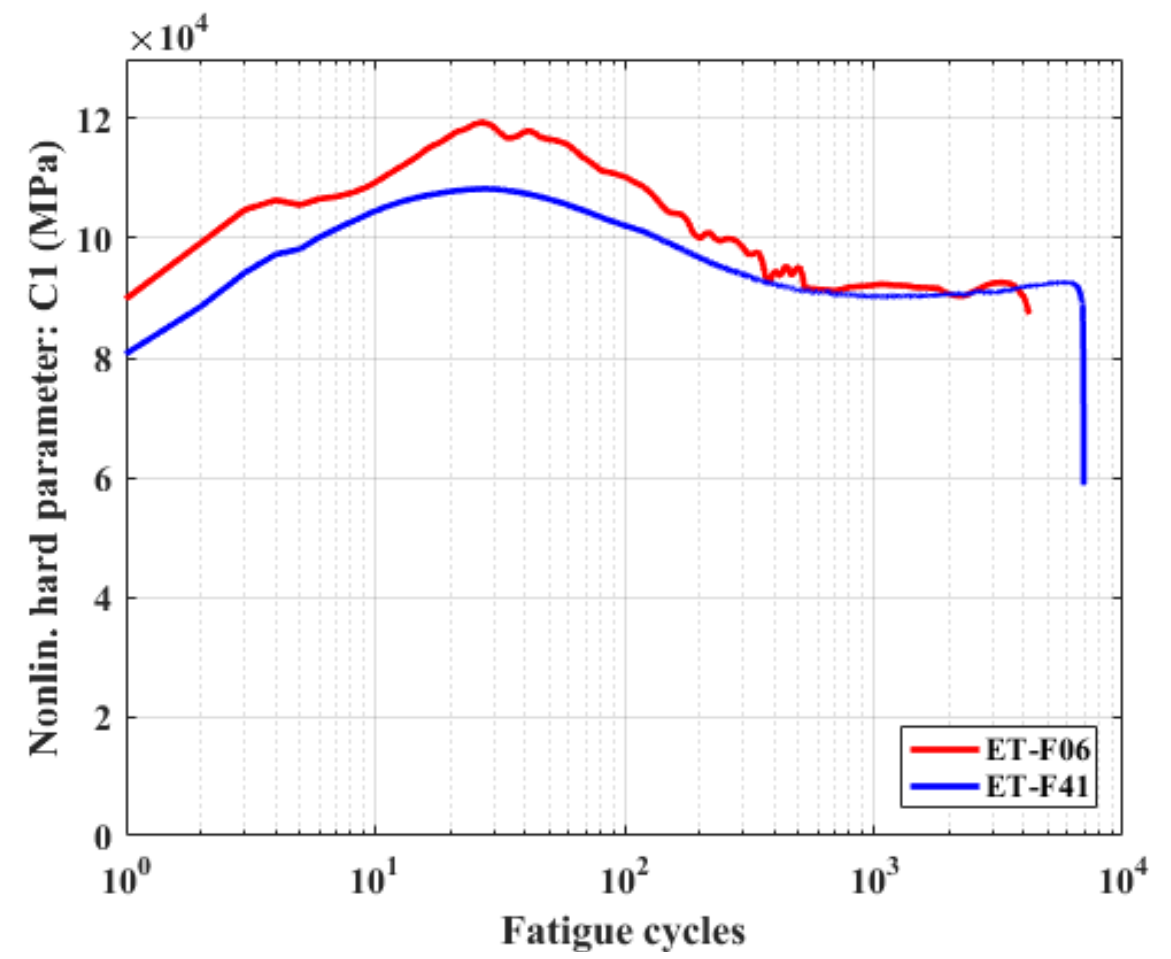

Figure 3. 8 Time/cycle-dependent nonlinear kinematic hardening parameter, $\mathrm{C} 1$ estimated from constant-amplitude fatigue tests.

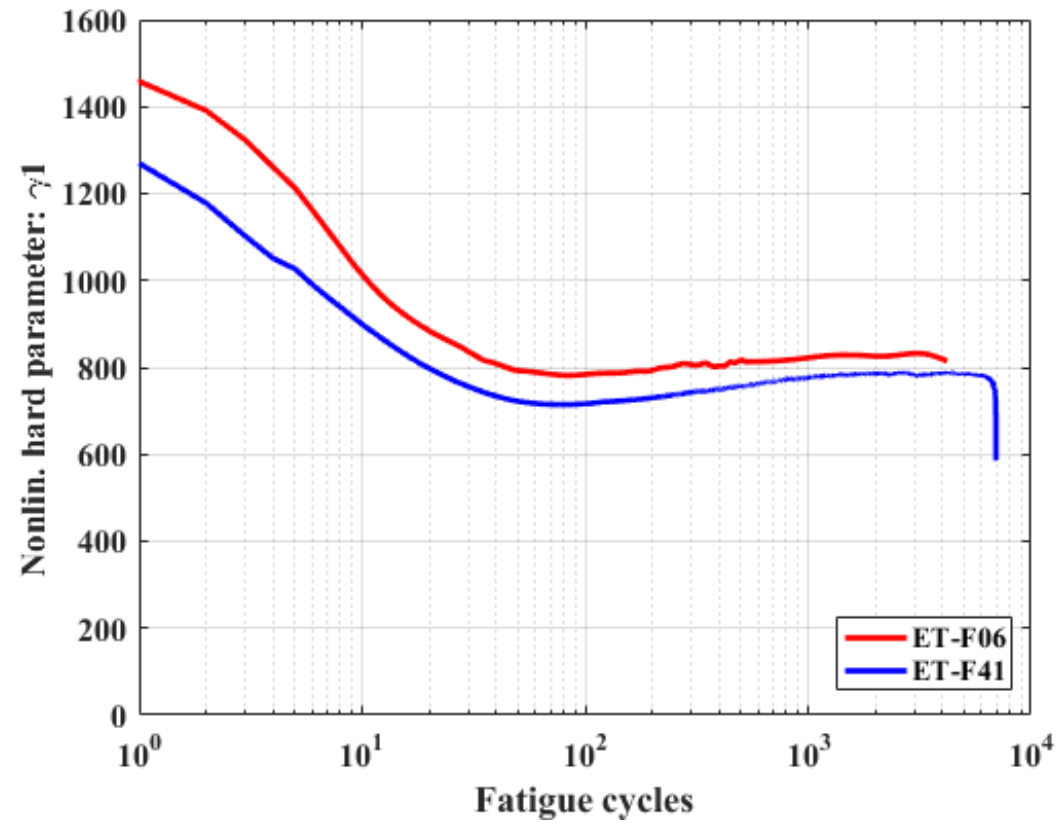

Figure 3. 9 Time/cycle-dependent nonlinear kinematic hardening parameter, $\gamma 1$ estimated from constantamplitude fatigue tests. 


\subsection{Variable-Amplitude Fatigue Test and Associated Cyclic Elastic-Plastic Material Model Results}

In our earlier work [22] we found that, although the constant-amplitude fatigue test can be used for time-dependent material properties, the estimated properties are sensitive to loading amplitude. To avoid the issues of amplitude dependency of material parameters, in our earlier work [26], we estimated material parameters for 508 low alloy steel (LAS) based on variable-amplitude fatigue test. In this report, we present fatigue test and associated material model results from variable-amplitude fatigue testing of a 316 SS specimen. The ultimate aim is to develop mechanistic models of real plant components subjected to random fatigue loading with different loading amplitudes.

Since it is not possible to perform constant-amplitude fatigue tests for each loading amplitude in a loading spectrum and then estimate the material properties for those loading amplitudes as separate constant-amplitude cases (as discussed in Section 3.2), we propose to estimate average material parameters based on a generic variable-amplitude fatigue test. Those average parameters then can be used for estimating the stress-strain state of a specimen/component subjected to any loading amplitude (constant or random) bounded within the loading spectrum and environmental conditions of the proposed variable-amplitude fatigue test. For the purpose, we conducted the ET-F38 variable-amplitude fatigue test at $300{ }^{\circ} \mathrm{C}$ and in air using a $316 \mathrm{SS}$ specimen. During ET-F38, a repetitive block consists of 12 cycles with different amplitudes being applied during the variable-amplitude fatigue experiment. The strain amplitude was varied by gradually increasing from a minimum value of $0.05 \%$ (selected to fall within the elastic limit, see Figure 3.1) to a maximum value of $0.55 \%$ and then gradually decreasing to the minimum again. Figure 3.10 depicts the applied cyclic strain input within a block during ET-F38. The corresponding time history of the measured stress data from ET-F38 is shown in Figure 3.11. As seen in the case of the constant-amplitude fatigue test, significant initial stress hardening followed by stress softening was observed during ET-F38. The stress-strain hysteresis plot for the $1^{\text {st }}$ block is shown in Figure 3.12. As seen in the figure, there are 12 cyclic stress-strain curves within a block. As there are several stress-strain cycles with different amplitudes within a block, the estimated time/block-dependent material parameters should capture the amplitude dependency of the parameters.

The Section 2 presents the block-dependent material parameter estimation technique. The method used for estimating the block-dependent parameters of 508 LAS is reported in [26]. Note that the cycledependent parameters discussed in Section 3.2 are different from the block-dependent parameters discussed in this section. The cycle-dependent parameters are estimated to capture the stress-strain state of a specimen subjected to single-loading amplitude, whereas the purpose of block-dependent parameters is to capture the stress-strain state of a material subjected to multiple loading amplitudes through a single test of average parameters at a given time.

An example comparison between experimental and predicted true back stress (using the estimated average parameters and analytical Chaboche equation) as a function of true plastic strain for a particular block (comprising all 12 cycles within that block) is shown in Figure 3.13. The estimated block-dependent elastic-plastic material parameters using ET-F38 test data are plotted in Figures 3.14 to 3.17. Figures 3.14 and 3.15 show the block variation of the estimated elastic modulus and elastic limit stress, respectively, while Figures 3.16 and 3.17 show the block variation of the estimated nonlinear kinematic hardening parameters $\mathrm{C} 1$ and $\gamma 1$, respectively. 
In addition to estimating appropriate elastic-plastic parameters, those parameters need to be described as a function of an appropriate field variable, which needs to be estimated by time/cycle/block-dependent mechanistic modeling. Among the possible choices for the field variable (that affects the fatigue damage process), the time/cycle/block is an obvious choice because of its easy tracking. However, direct tracking of the time/cycle/block can only be useful for modeling a test case using the parameters estimated from the same test case. As discussed earlier in Section 2, describing material behavior with respect to time/cycle/block may not allow selection of an appropriate set of parameters for a modeling test case, if the parameters are estimated from a different fatigue test. Hence, the estimated parameters must be described with respect to a physical/field variable different from the time/cycle/block.

In this report, we propose a parameter mapping approach based on APSE. The APSE-based modeling approach is discussed in detail in Section 2. In this approach, the calculated APSE at the end of a time step is used to select the material parameters for predicting the stress-strain in the next step. The material parameters estimated from variable-amplitude test ET-F38 were used for APSE-based modeling. Figures 3.18 to 3.21 show the material parameters estimated from ET-F38 as functions of APSE. These functions were used to map the material parameters between the variable-amplitude test and the test being modeled.

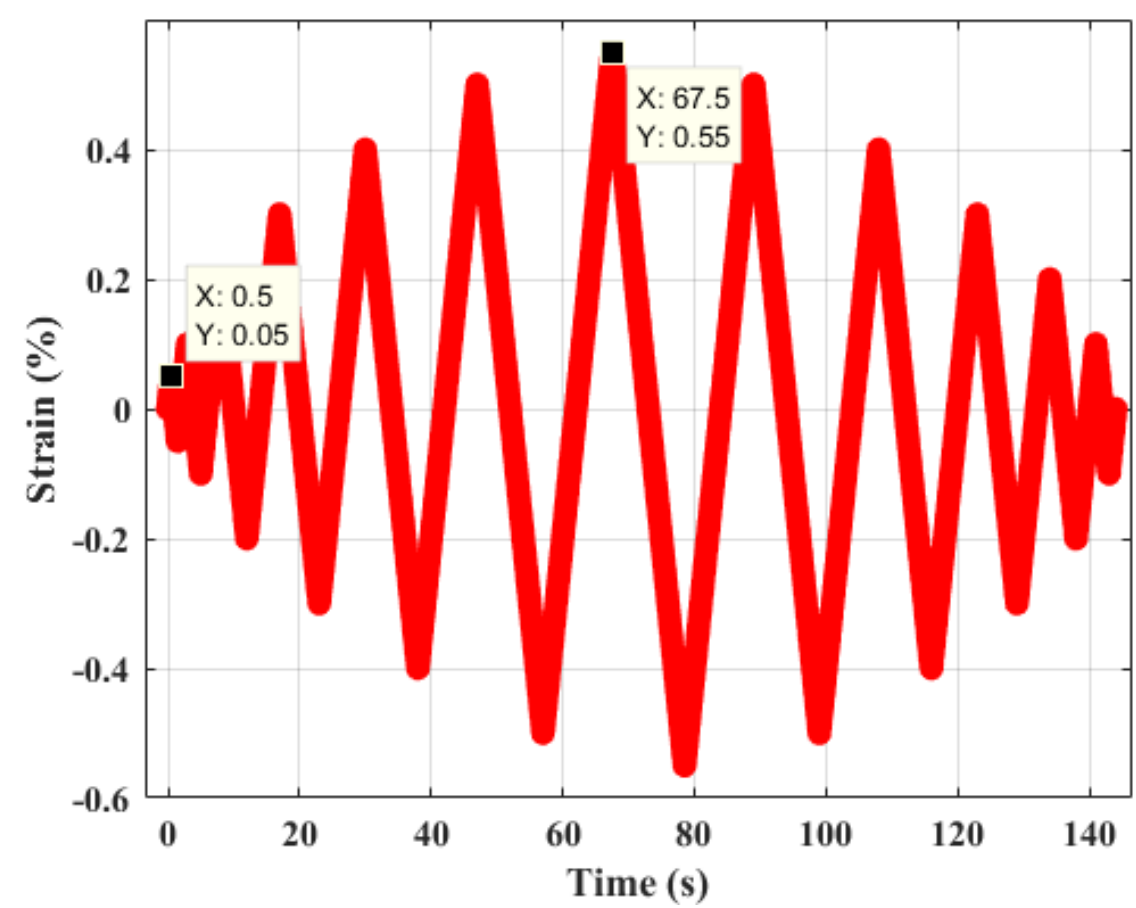

Figure 3. 10 Block loading during variable-amplitude strain-controlled fatigue test (ET-F38). Data points show minimum and maximum amplitudes. 


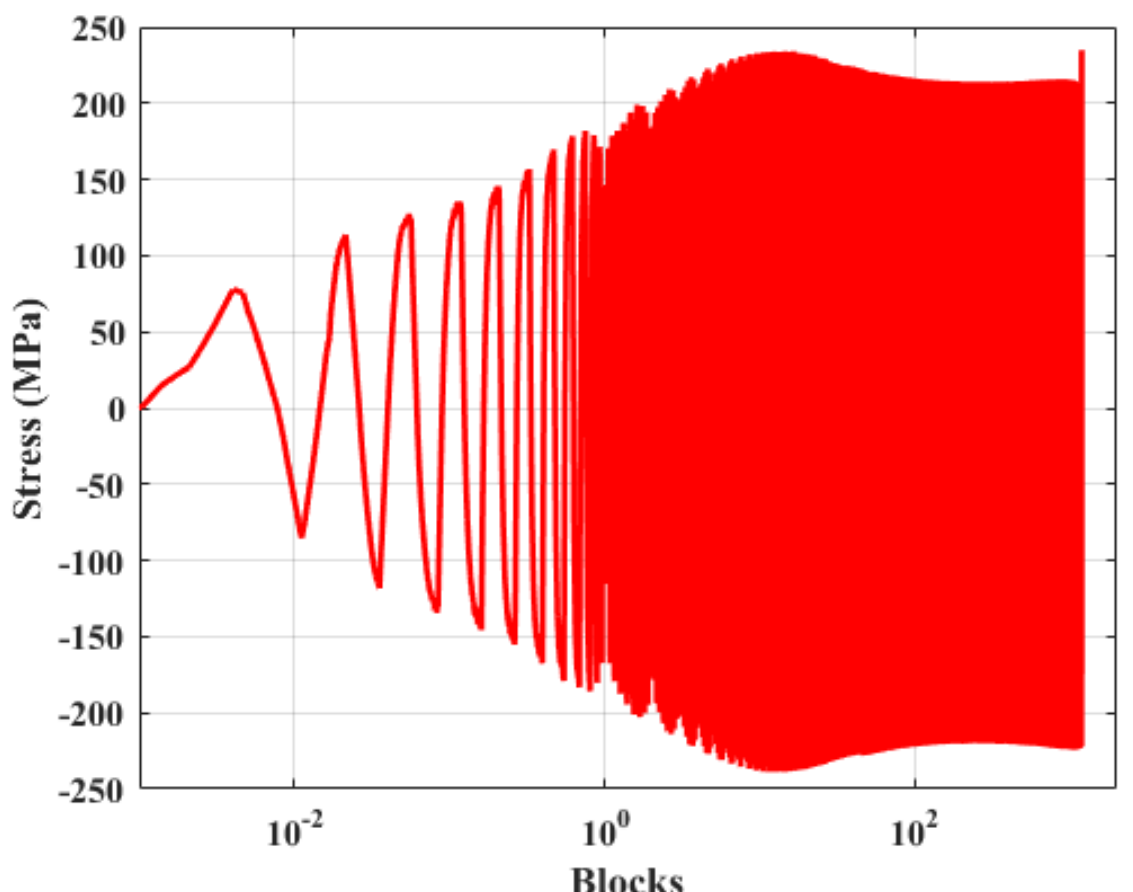

Figure 3. 11 Observed stress during the entire ET-F38 fatigue test.

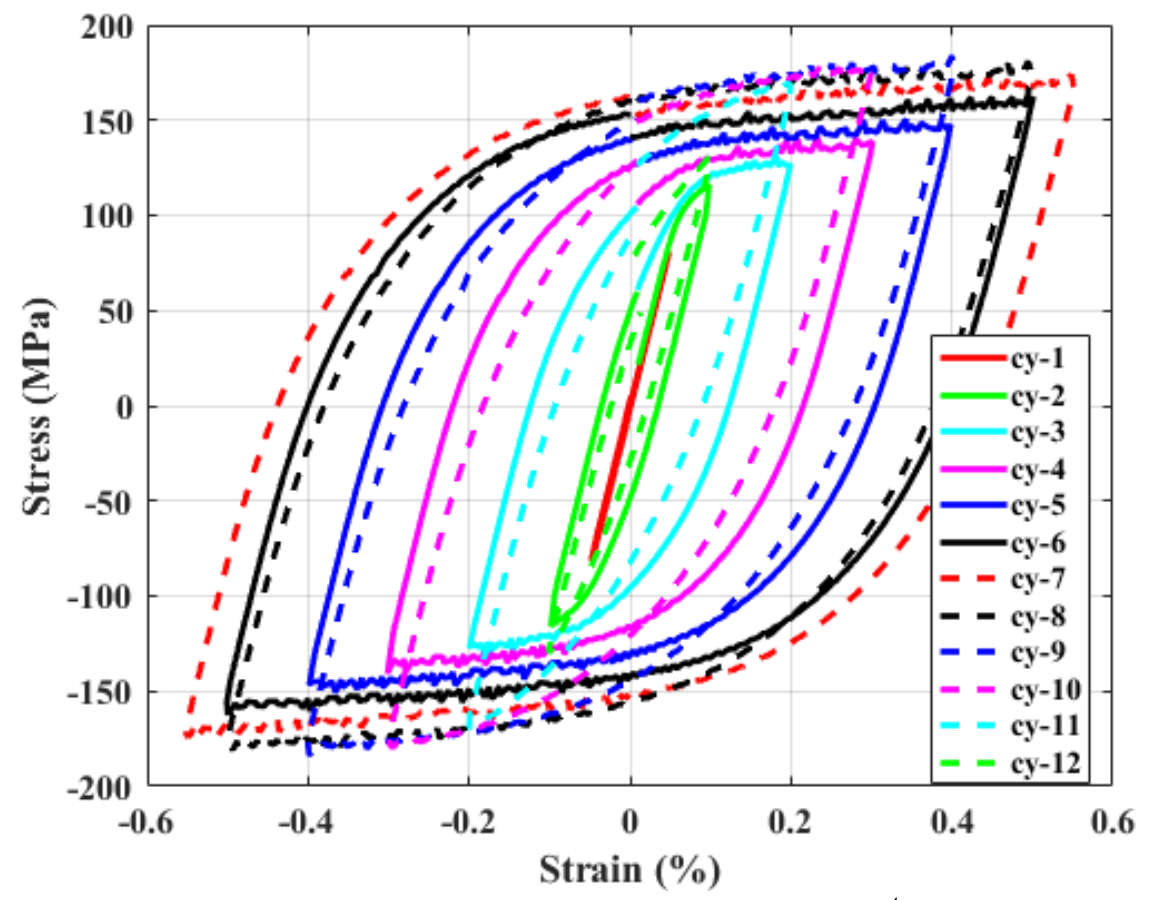

Figure 3. 12 Observed stress-strain hysteresis behavior during the $1^{\text {st }}$ block (each block comprising 12 cycles) of ET-F38. 


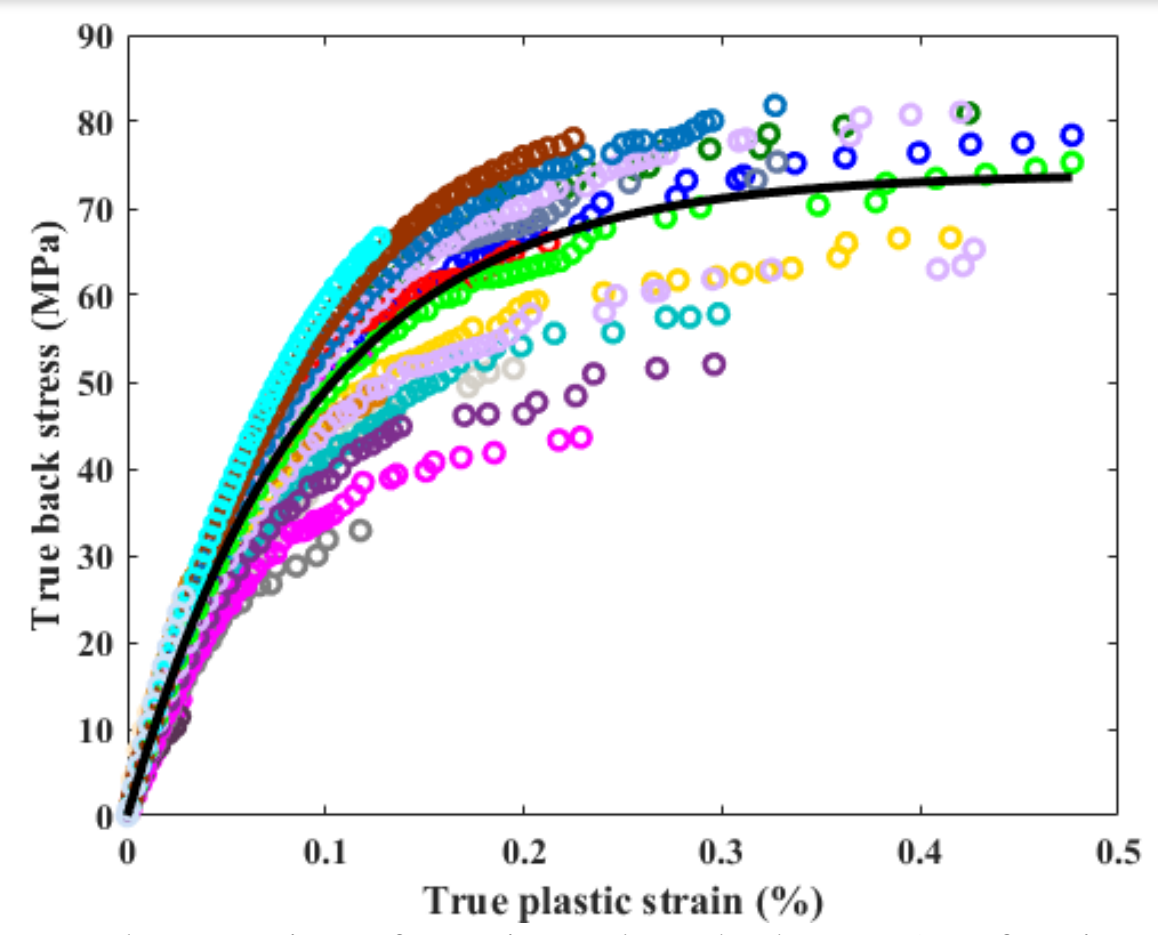

Figure 3. 13 Example comparison of experimental true back stress (as a function of true plastic strain) and predicted back stress (using kinematic hardening equation and estimated parameters: $\mathrm{C} 1$ and $\gamma 1$ pertaining to that block). Black solid line: prediction; Circles: experimental data from 12 fatigue cycles of that block.

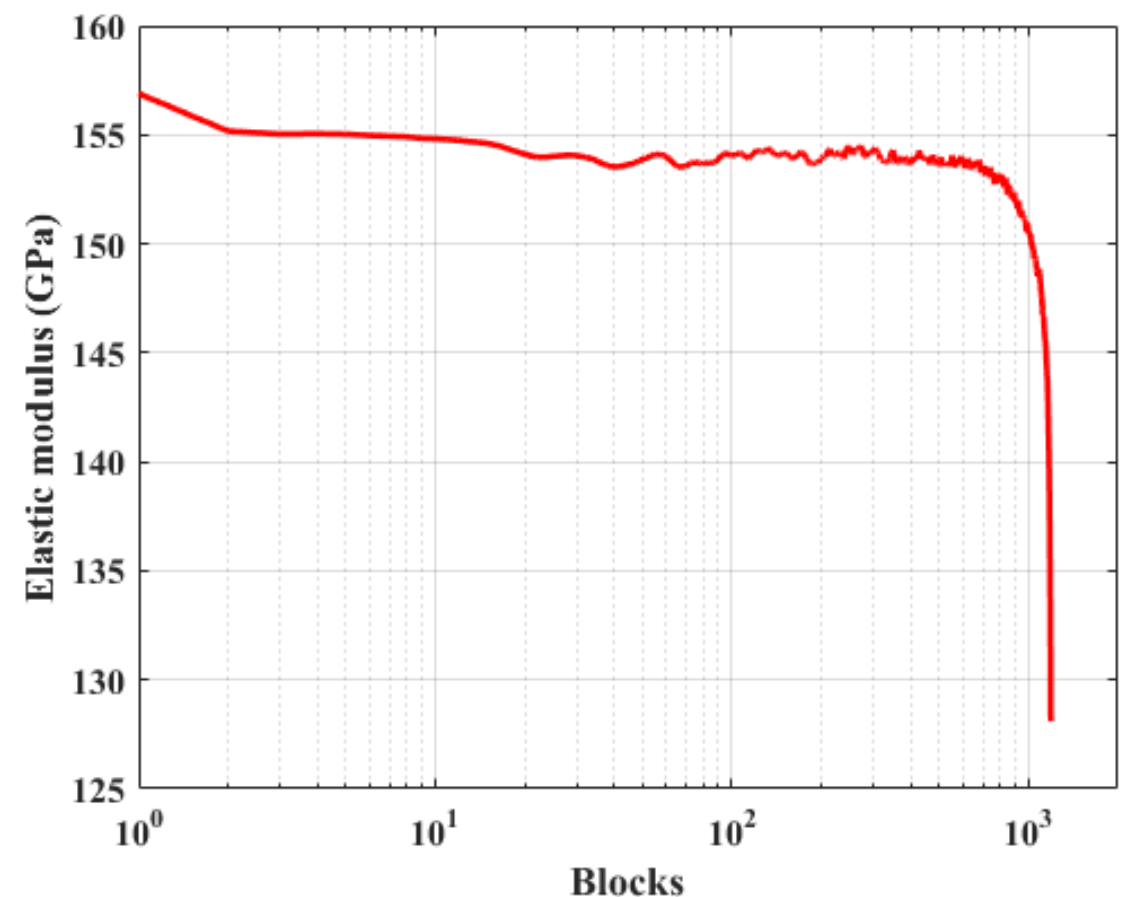

Figure 3. 14 Time/block-dependent elastic modulus estimated from variable-amplitude fatigue test (ETF38). 


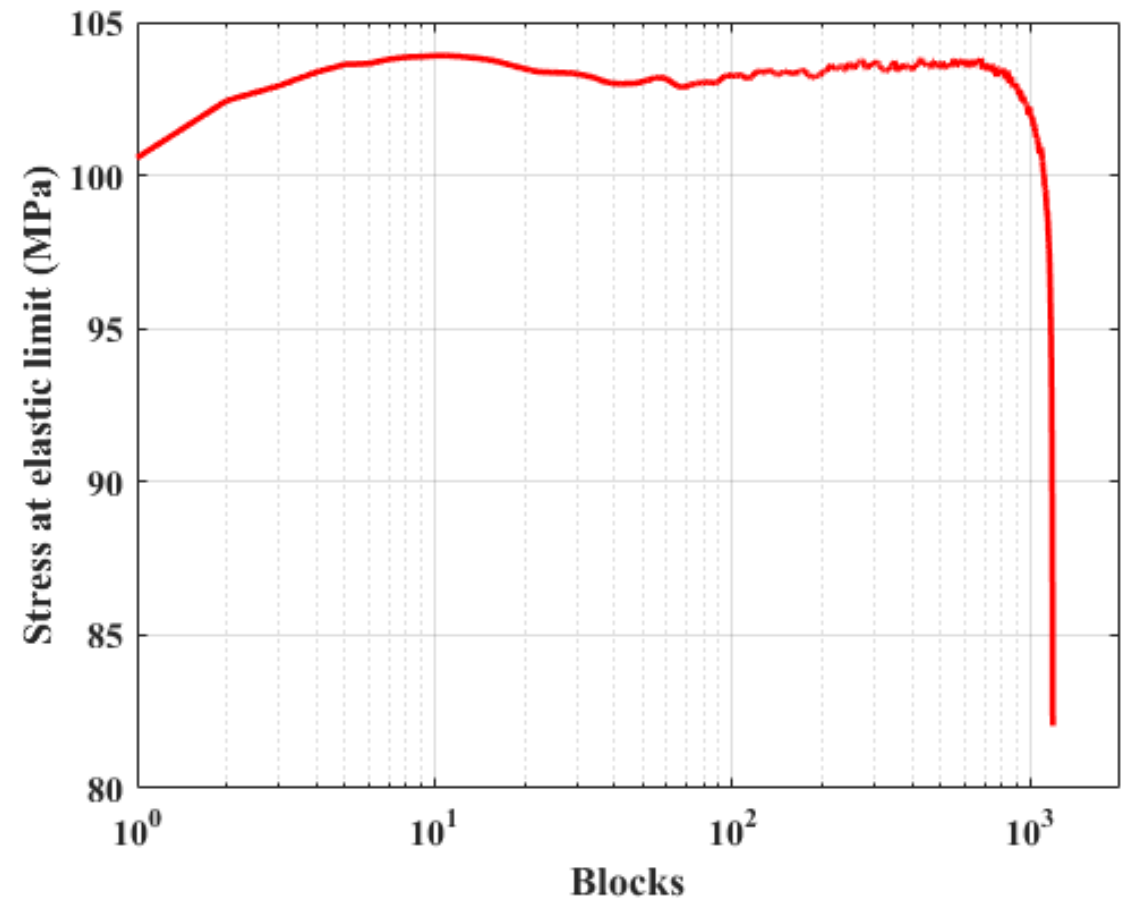

Figure 3. 15 Time/block-dependent elastic limit stress estimated from variable-amplitude fatigue test (ET-F38).

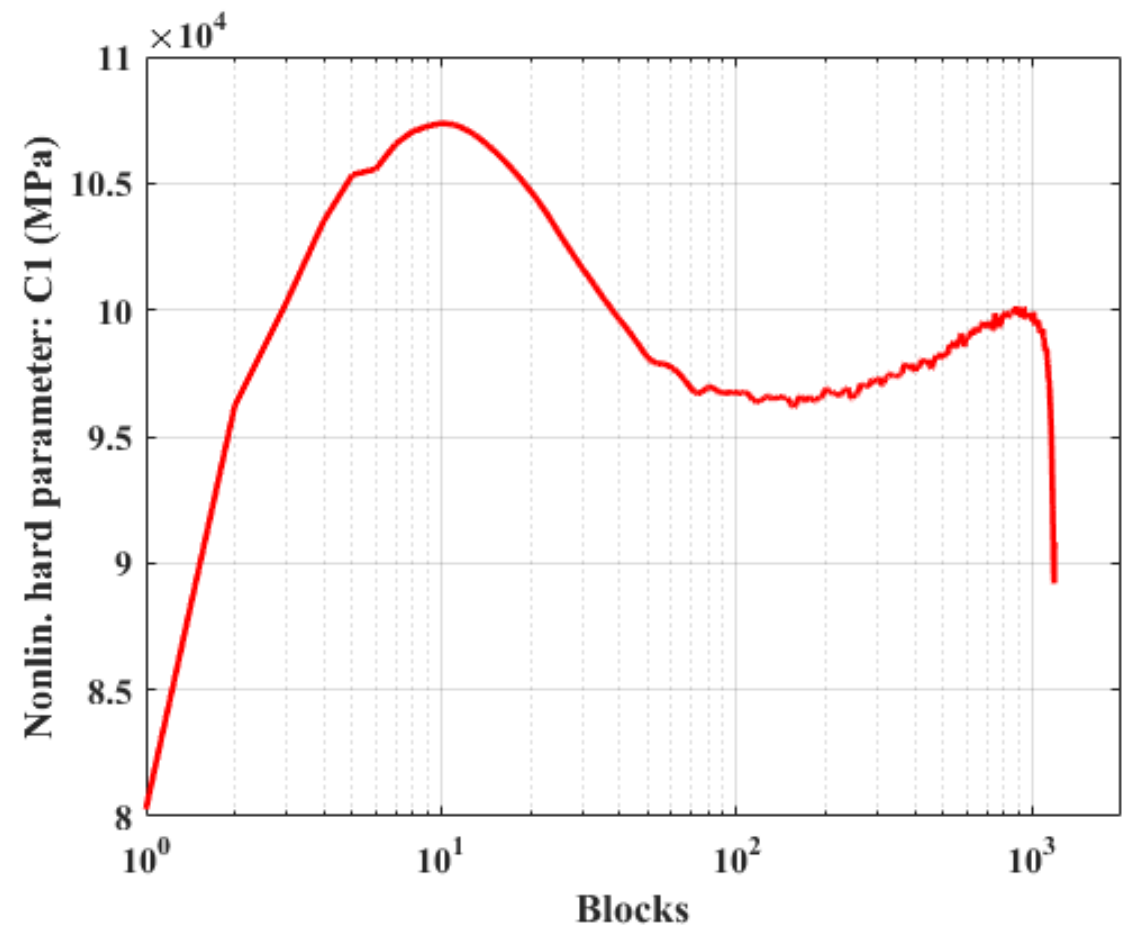

Figure 3. 16 Time/block-dependent nonlinear kinematic hardening parameter, $\mathrm{C} 1$ estimated from variable-amplitude fatigue test (ET-F38). 


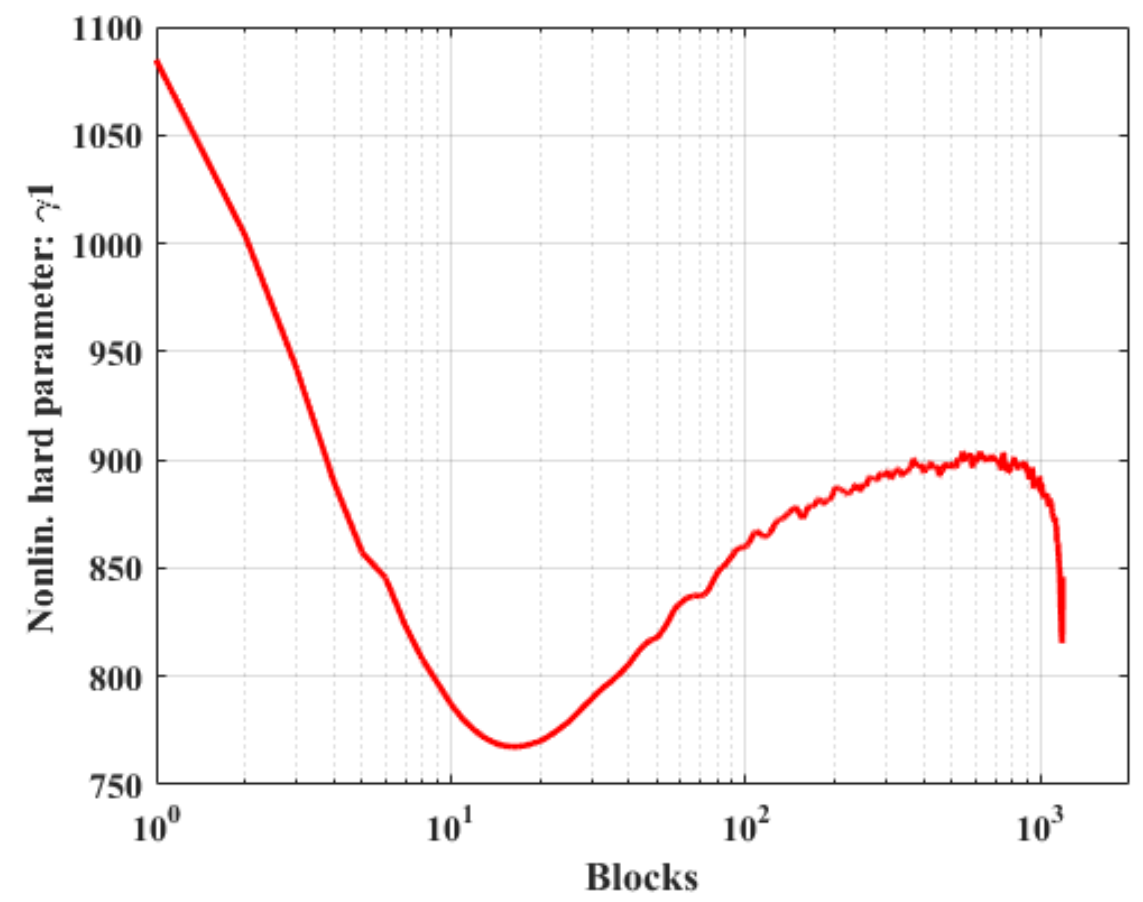

Figure 3. 17 Time/block-dependent nonlinear kinematic hardening parameter, $\gamma 1$ estimated from variable-amplitude fatigue test (ET-F38).

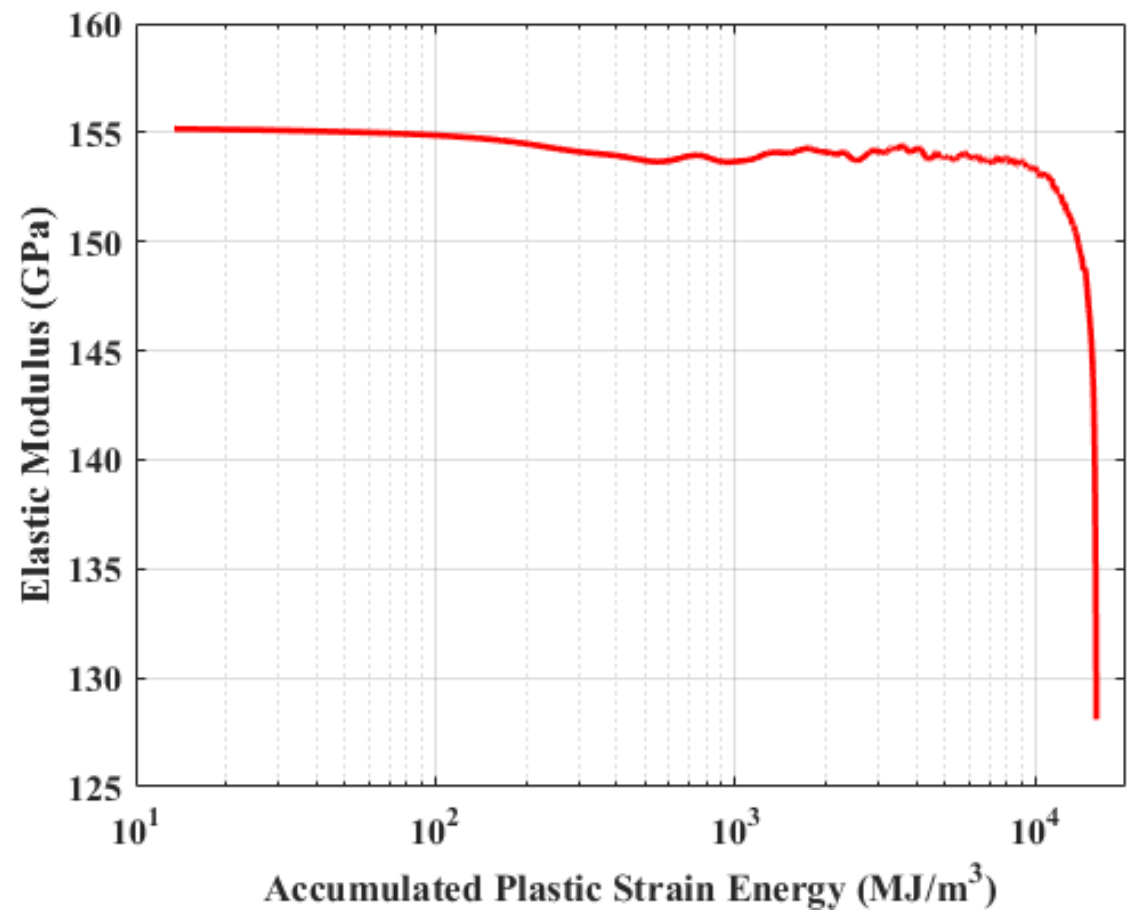

Figure 3.18 APSE-dependent elastic modulus estimated from variable-amplitude fatigue test (ET-F38). The $\mathrm{y}$-data corresponding to $\mathrm{x}=0$ is ignored in the semi-logx plot. 


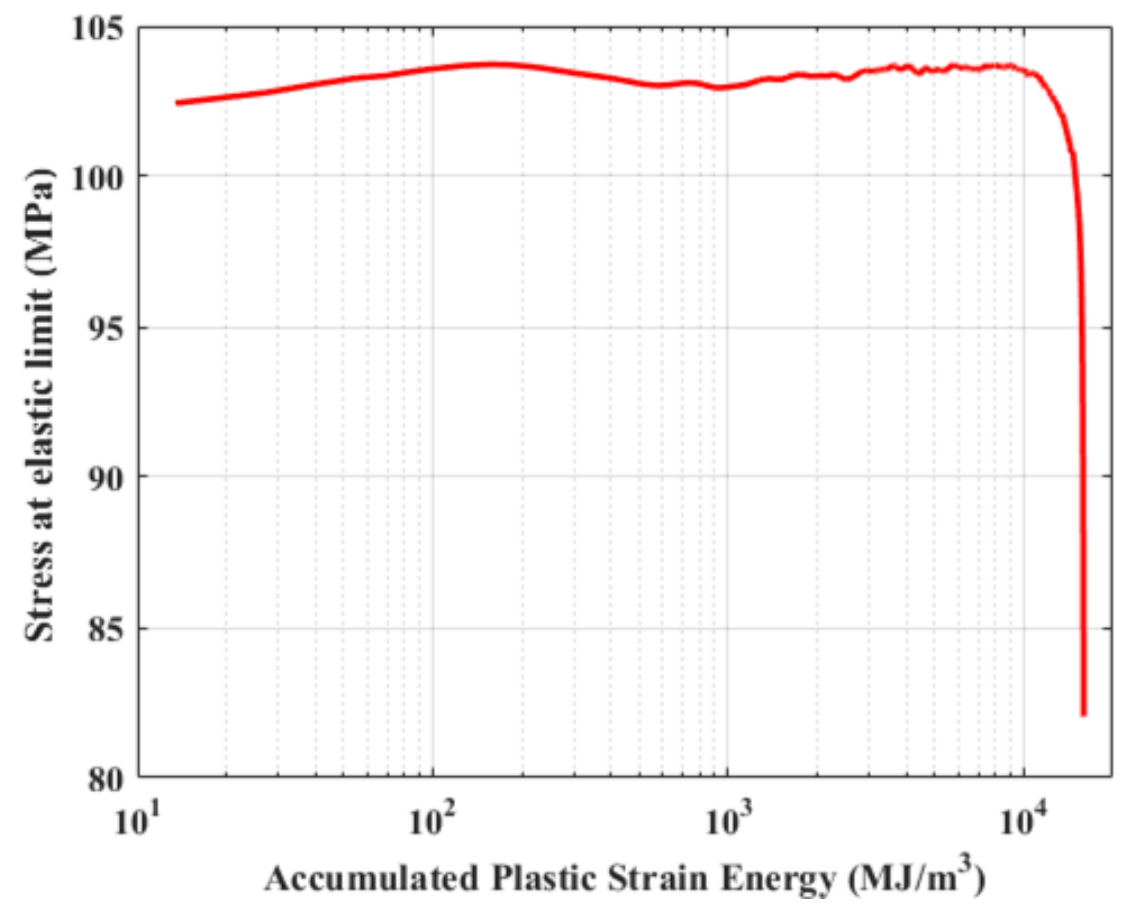

Figure 3.19 APSE -dependent elastic limit stress estimated from variable-amplitude fatigue test (ETF38). The $y$-data corresponding to $\mathrm{x}=0$ is ignored in the semi- $\log \mathrm{x}$ plot.

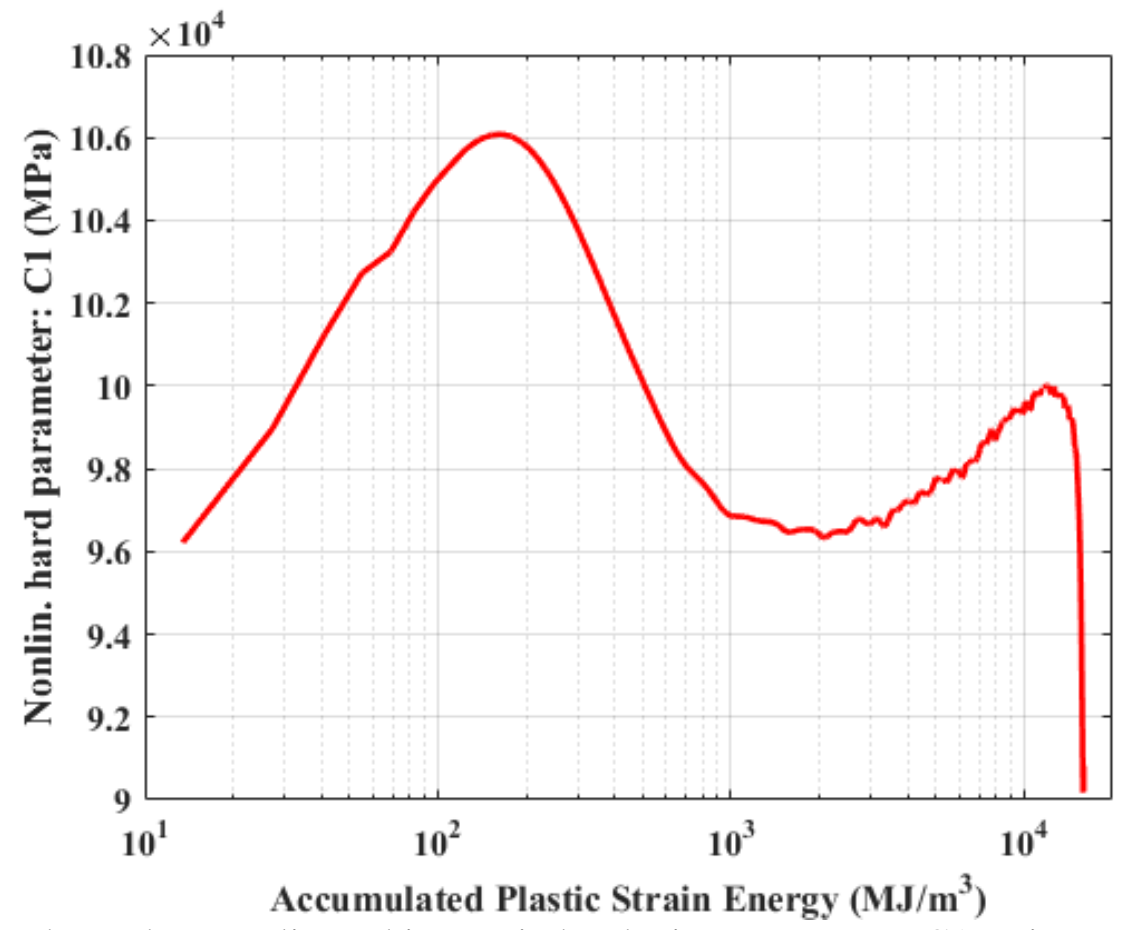

Figure 3. 20 APSE -dependent nonlinear kinematic hardening parameter, $\mathrm{C} 1$ estimated from variableamplitude fatigue test (ET-F38). The $y$-data corresponding to $\mathrm{x}=0$ is ignored in the semi- $\log \mathrm{x}$ plot. 


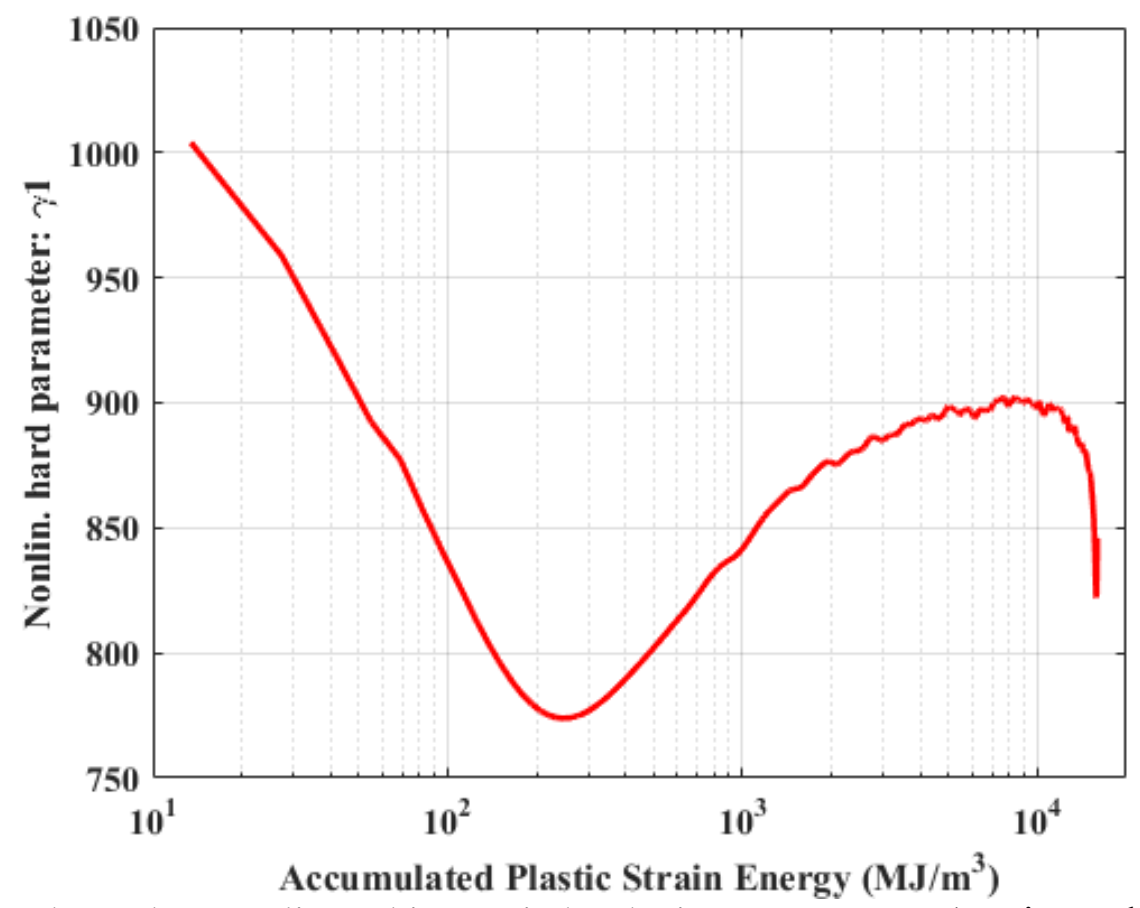

Figure 3. 21 APSE-dependent nonlinear kinematic hardening parameter, $\gamma 1$ estimated from variableamplitude fatigue test (ET-F38). The $\mathrm{y}$-data corresponding to $\mathrm{x}=0$ is ignored in the semilogx plot.

\subsection{Random-Amplitude Fatigue Test Results}

Nuclear reactor structures encounter random complex loading during their service life due to random temperature and pressure transients. Thus, developing a fatigue model that can capture material behavior under random loading is essential for accurate life prediction of nuclear reactor components. With this in mind, we conducted a strain-controlled random-amplitude test fatigue test (ET-F40). The aim of the test was to develop a validated model to predict the material stress-strain state under random loading. The random test was conducted for validation purposes only. For modeling the random load case, we used the parameters estimated based on the variable loading test ET-F38. The respective analytical and 3D-FE modeling results will be discussed in Sections 4 and 5. Here we discuss a few results directly related to the fatigue test.

During the random-amplitude fatigue test (ET-F40), a repetitive block of random strain inputs was applied to the specimen. The applied random strain input within a block is shown in Figure 3.22. As seen from the figure, the strain inputs were bounded with a maximum $/$ minimum strain amplitude of $\pm 0.55 \%$, the same as the bounded strain inputs for the variable-amplitude test (ET-F38). The in-between strain inputs were selected based on a MATLAB-based random number generator. The observed stress history for the entire test ET-F40 is shown in Figure 3.23. As seen in the figure, there was substantial initial stress hardening followed by stress softening for the 316 SS under the random-amplitude strain-controlled test. Similar behavior was observed for the constant-amplitude (ET-F06 and ET-F41) and variable-amplitude (ET-F38) strain-controlled tests. To better check the randomness of the applied strain, Figure 3.24 presents a magnified (in time axis) plot of strain vs. time. The corresponding observed stress and hysteresis plots 
are shown in Figures 3.25 and 3.26, respectively. As seen in Figure 3.26, there are many small hysteresis loops inside the big hysteresis loop. However, these loops are not symmetric about the x- and y-axes, as observed for the variable-amplitude test case (see Figure 3.12). Thus, modeling fatigue behavior under random-amplitude loading is a complex problem.

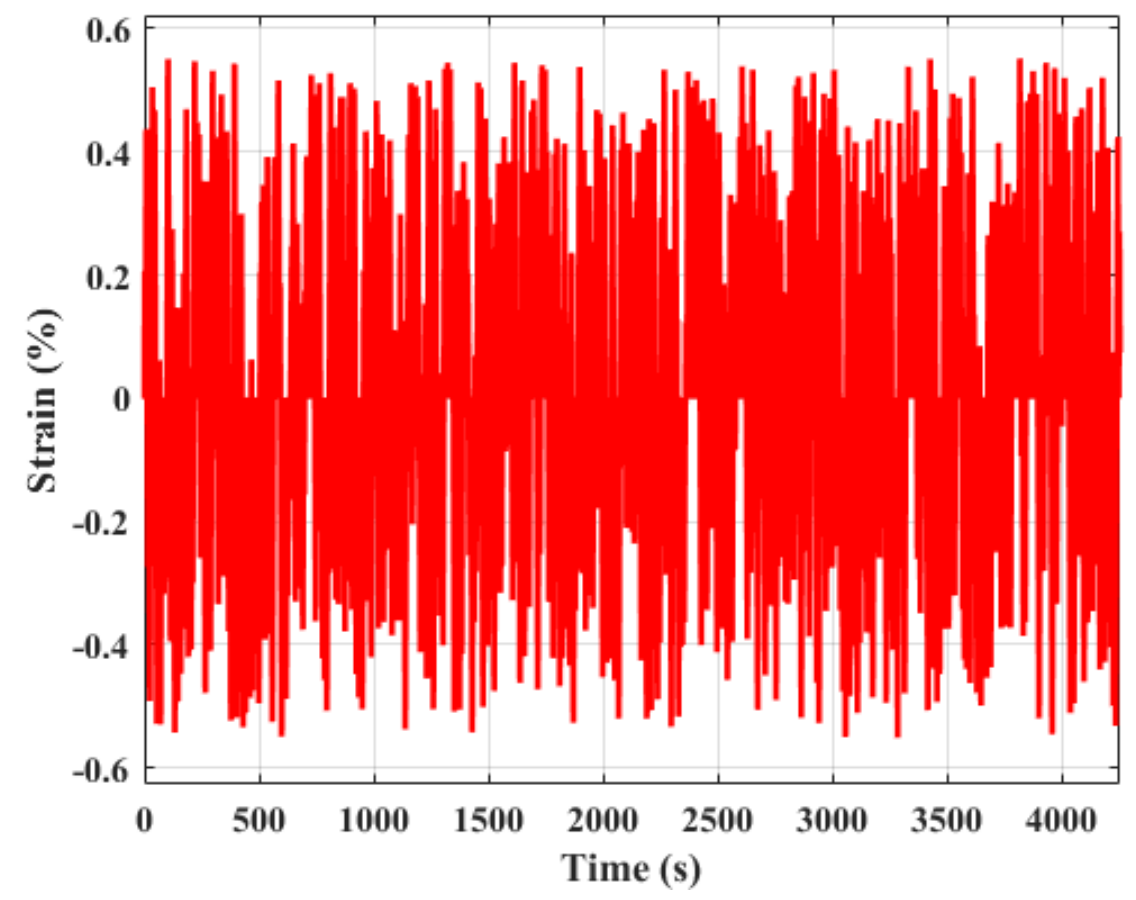

Figure 3. 22 Block loading during random-amplitude strain-controlled fatigue test (ET-F40).

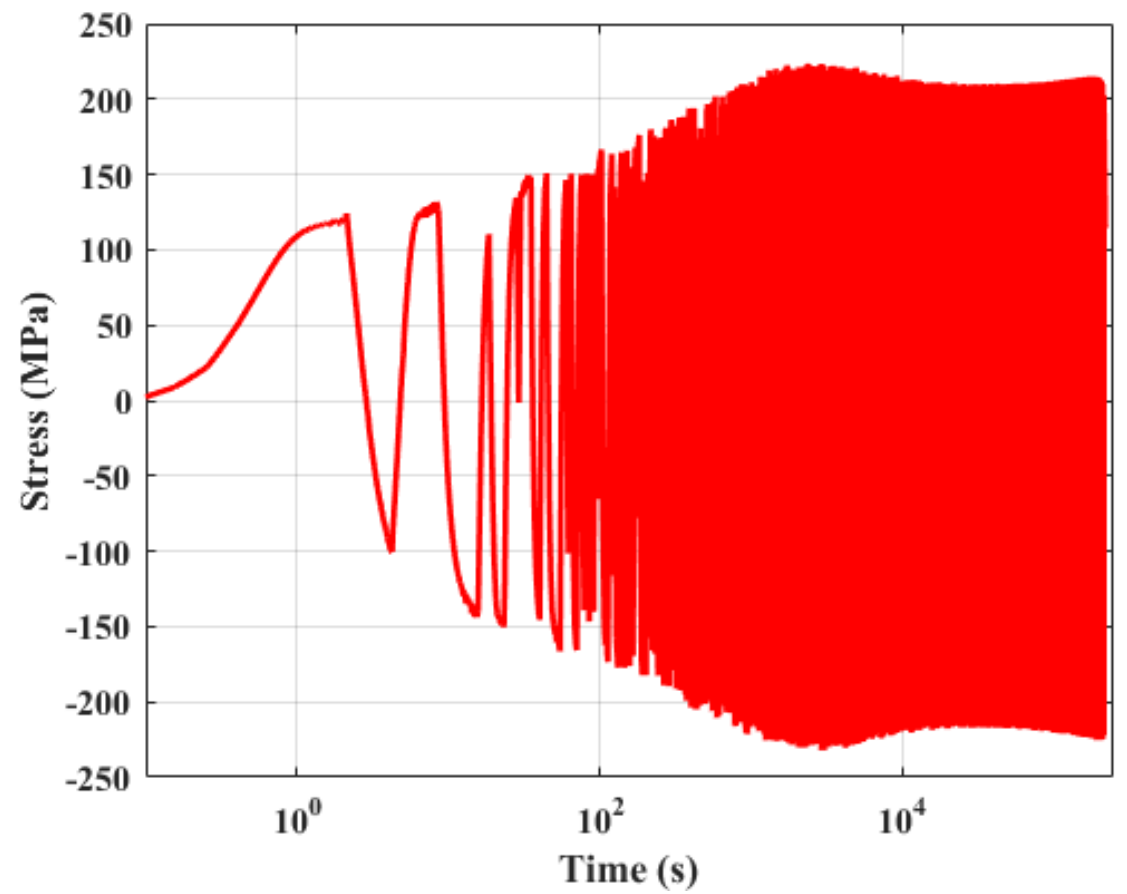

Figure 3. 23 Observed stress during the entire ET-F40 fatigue test. 


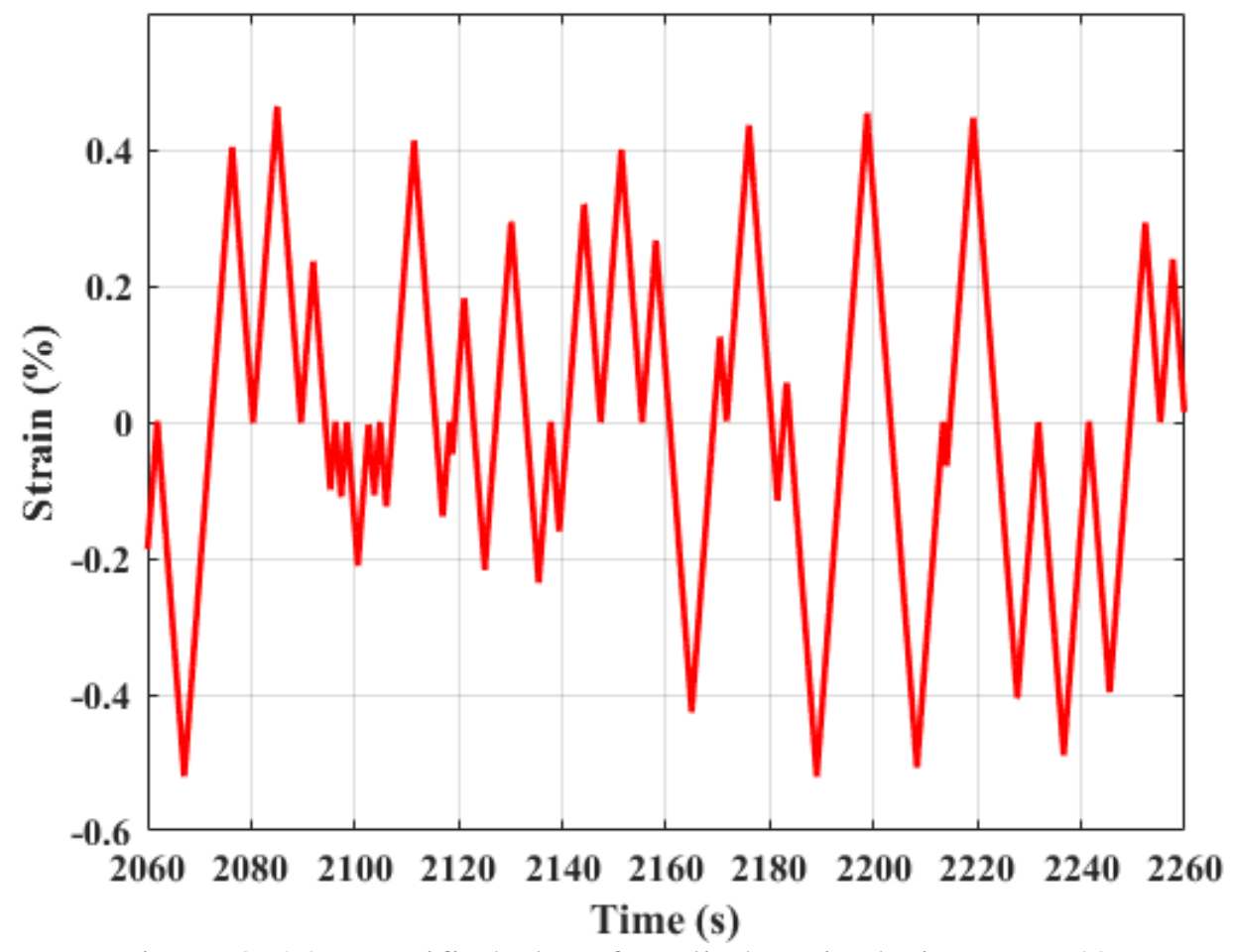

Figure 3. 24 Magnified plot of applied strain during ET-F40.

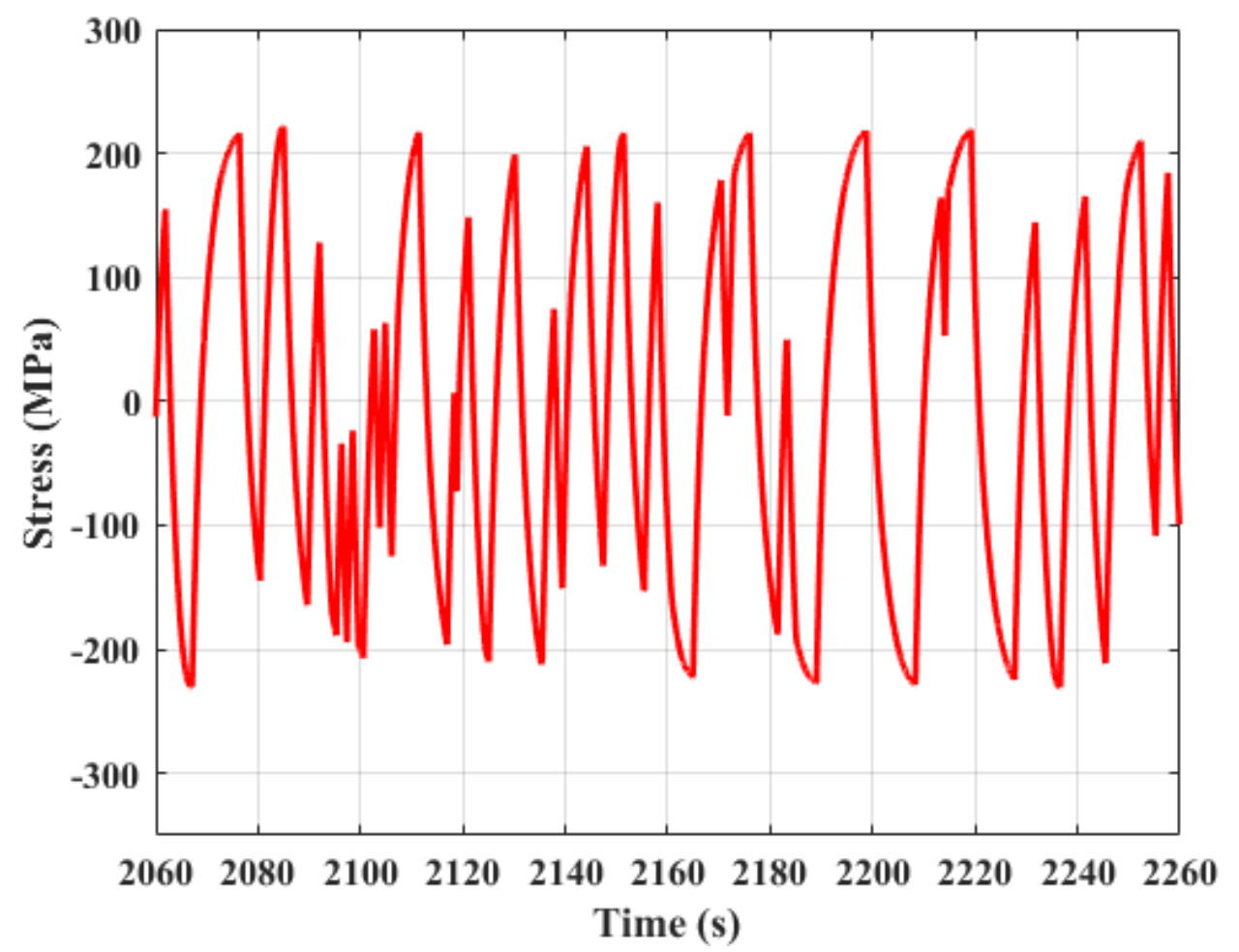

Figure 3. 25 Magnified plot of observed stress (corresponding to strain shown in Figure 3.24) during ET-F40. 


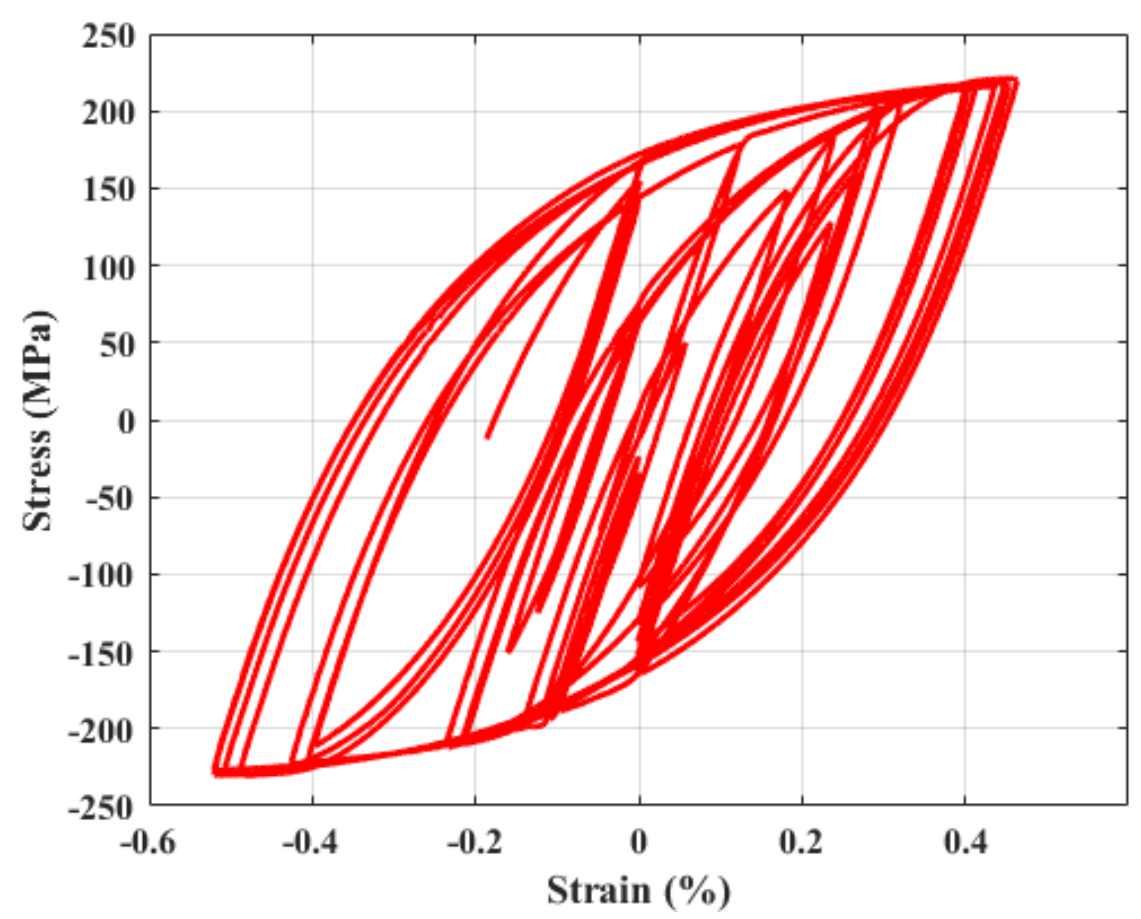

Figure 3. 26 Example hysteresis plot of stress (shown in Figure 3.25) and strain (shown in Figure 3.24) during ET-F40. 


\section{Results from Analytical Modeling of Constant, Variable, and Random Load Fatigue Tests}

Our evolutionary cyclic plasticity model along with related material parameter estimation technique is described in Section 2. Section 2 also describes a time- and APSE-based material parameter mapping approach during modeling of the fatigue tests. A time-based approach can be used when a constant- or variable-amplitude loading is applied but cannot be used to model fatigue behavior under random loading. To solve this issue, a more versatile approach based on APSE is proposed. The APSE-based modeling can be used to model fatigue behavior under any loading condition (constant, variable, or random). In this section, results from time-based analytical modeling of constant-amplitude (ET-F06 and ET-F41) and variable-amplitude (ET-F38) tests are presented. Results from APSE-based analytical modeling of constant, variable, and random (ET-F40) amplitudes are also presented. Note that, for the APSE-based modeling, the material parameters estimated from the variable-amplitude test (ET-F38) were used for predicting the different test cases. All the tests were also modeled by using fixed material parameters estimated from the tensile test (ET-T04) and parameters at the half-life of the fatigue test specimen. All the fixed, time-dependent, and APSE-dependent material parameters are presented in Section 3.

\subsection{Results from Time-Based Analytical Models}

\subsubsection{Constant-Amplitude Fatigue Test}

Figure 4.1 presents experimentally observed and analytically modeled stress results for the constantamplitude fatigue test ET-F41. The figure shows predicted stress using time/cycle-dependent material parameters estimated from ET-F41 and two sets of time-independent or fixed parameters estimated from the tensile test ET-T04 and the half-life cycle of ET-F41. Three magnified versions of Figure 4.1 are shown in Figures 4.2 to 4.4, representing various important stages of material behavior during the specimen's fatigue life.

Figure 4.2 represents the initial hardening behavior of the material. The data indicate that the evolutionary cyclic plasticity model based on time/cycle-dependent material properties predicted the material hardening behavior with excellent accuracy, but that the model based on time-independent material properties estimated from tensile test ET-T04 and half-life cycle of ET-F41 test did not predict the material hardening behavior. Note that the tensile-test-based material parameter predicts this behavior well only up to the $1^{\text {st }}$ quarter cycle, as expected. The experimental condition up to the $1^{\text {st }}$ quarter cycle is similar to a tensile test, and after that a reverse loading is applied, and material behavior is more influenced by cyclic-loading-related reversals.

Figure 4.3 represents the softening behavior (after initial hardening) followed by stabilized cycles. As seen in the curves, the evolutionary cyclic plasticity model predicts both softening and stabilized cycle behavior of the material well. The prediction of stabilized cycles is found be good when half-life material properties are used. This finding is expected as the half-life cycle falls in the stabilized region, and material behavior does not change significantly in this region. 
Figure 4.4 represents the most important stage of a specimen in the context of fatigue life and final failure of a specimen. In this stage, stress drops at a high rate, and eventually, the specimen breaks in a way characteristic of unstable or rapid crack propagation. An accurate prediction of this stage is essential to determine the life of the specimen and, therefore, the life times of the components. Figure 4.4 also shows that the evolutionary cyclic plasticity model can accurately predict the last and most important stage of material behavior under constant-amplitude fatigue loading.

Similarly, another constant-amplitude fatigue test (ET-F06) was analytically modeled with the time/cycle-dependent material parameters estimated from ET-F06 and two sets of time-independent or fixed parameters estimated from the tensile test ET-T04 and the half-life cycle of ET-F06. The experimental and simulated stress is compared in Figure 4.5. As was the case for modeling the ET-F41 test, the evolutionary cycle plasticity model based on time/cycle-dependent material parameters can accurately predict all the stages of material behavior during the fatigue life of the specimen.

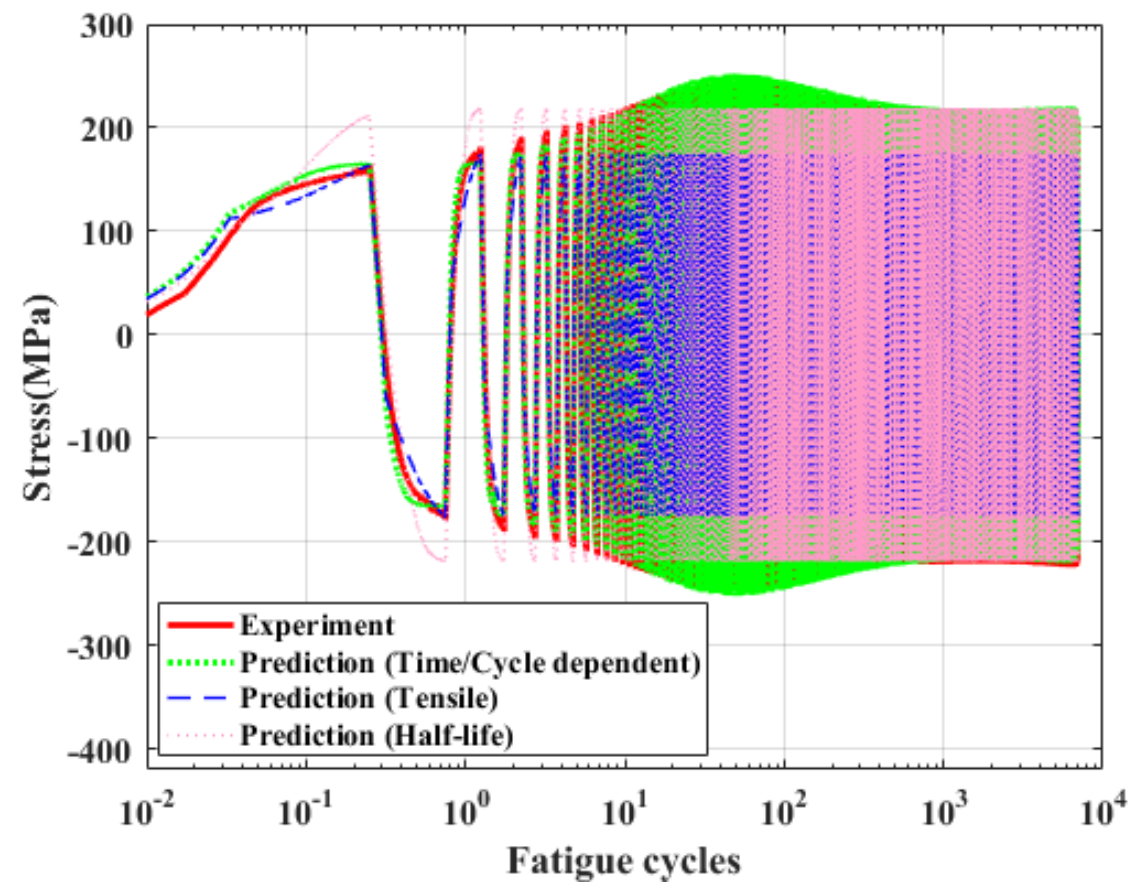

Figure 4. 1 Simulated (analytical) vs experimental axial stress history of ET-F41. Predictions are from simulation using time/cycle-dependent parameters (ET-F41) and two sets of time-independent parameters (tensile test ET-T04 and half-life cycle of ET-F41). 


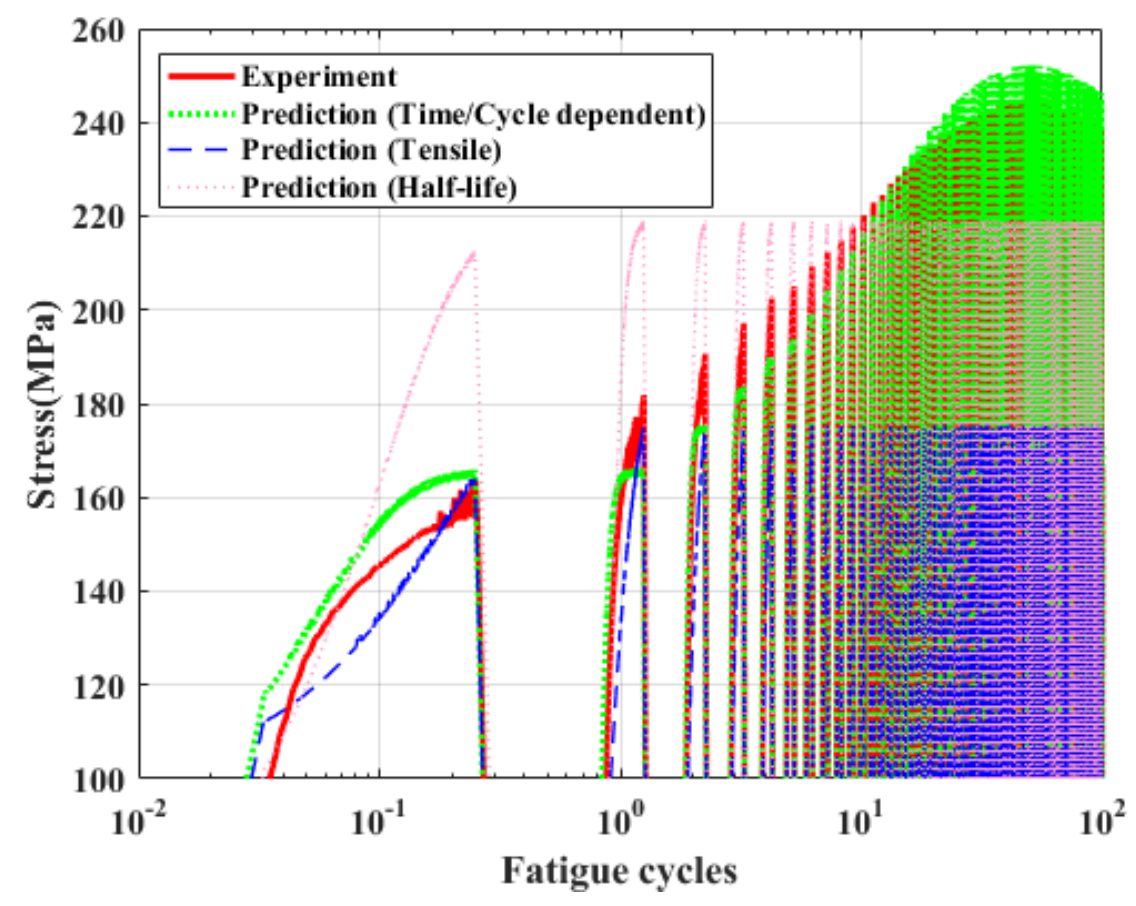

Figure 4. 2 Magnified version of Figure 4.1 showing the ability of evolutionary cyclic plasticity model (time/cycle-dependent prediction) to predict initial hardening behavior of $316 \mathrm{SS}$ under constantamplitude loading.

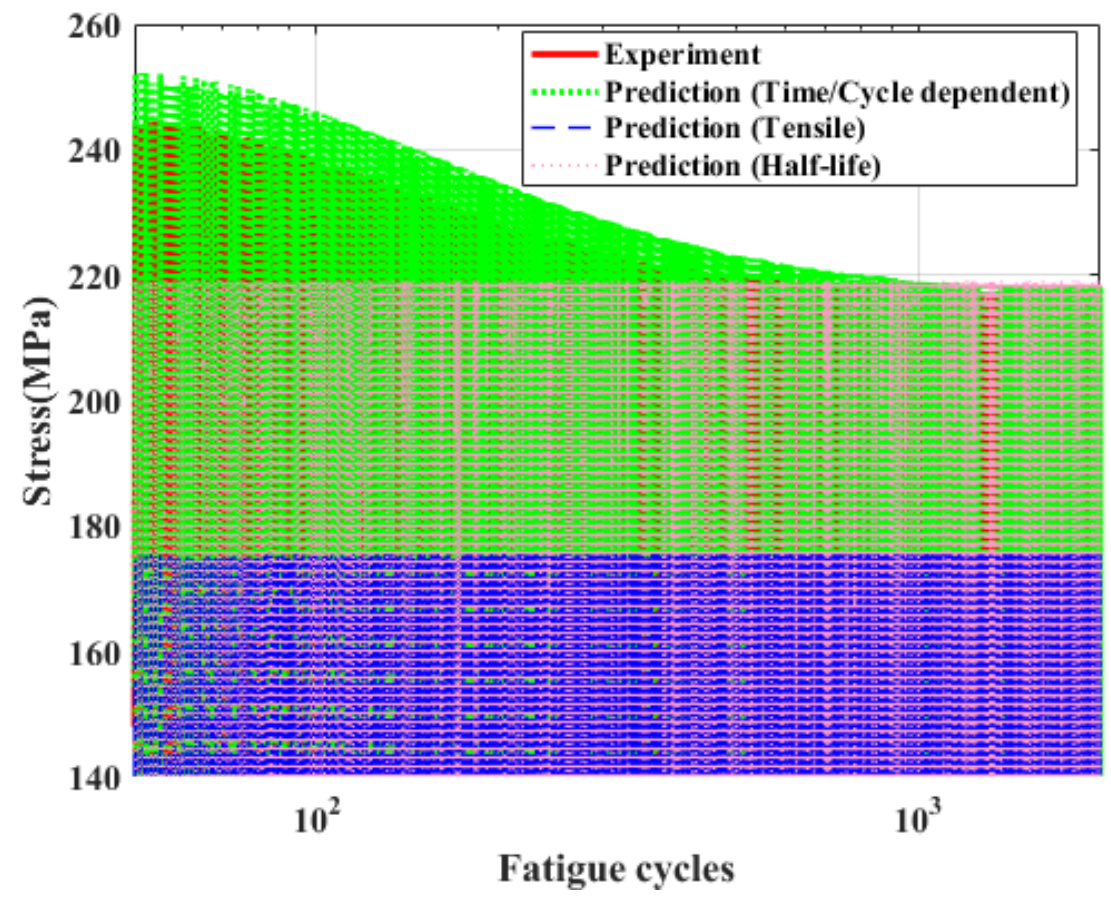

Figure 4. 3 Magnified version of Figure 4.1 showing the ability of evolutionary cyclic plasticity model (time/cycle-dependent prediction) to predict softening behavior of $316 \mathrm{SS}$ under constantamplitude loading. 


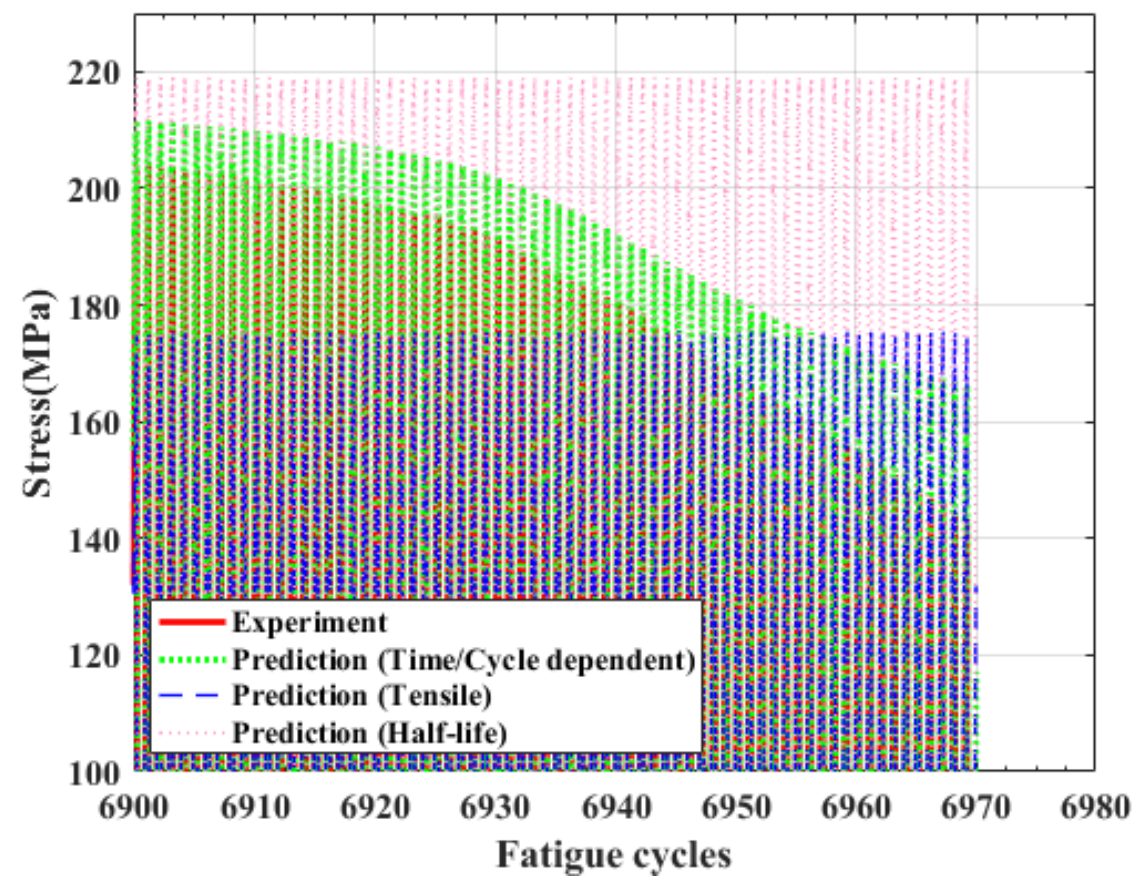

Figure 4. 4 Magnified version of Figure 4.1 showing the ability of evolutionary cyclic plasticity model (time/cycle-dependent prediction) to predict the fast stress drop toward the end of the fatigue life of specimen, representing unstable or rapid crack propagation, under constant-amplitude loading.

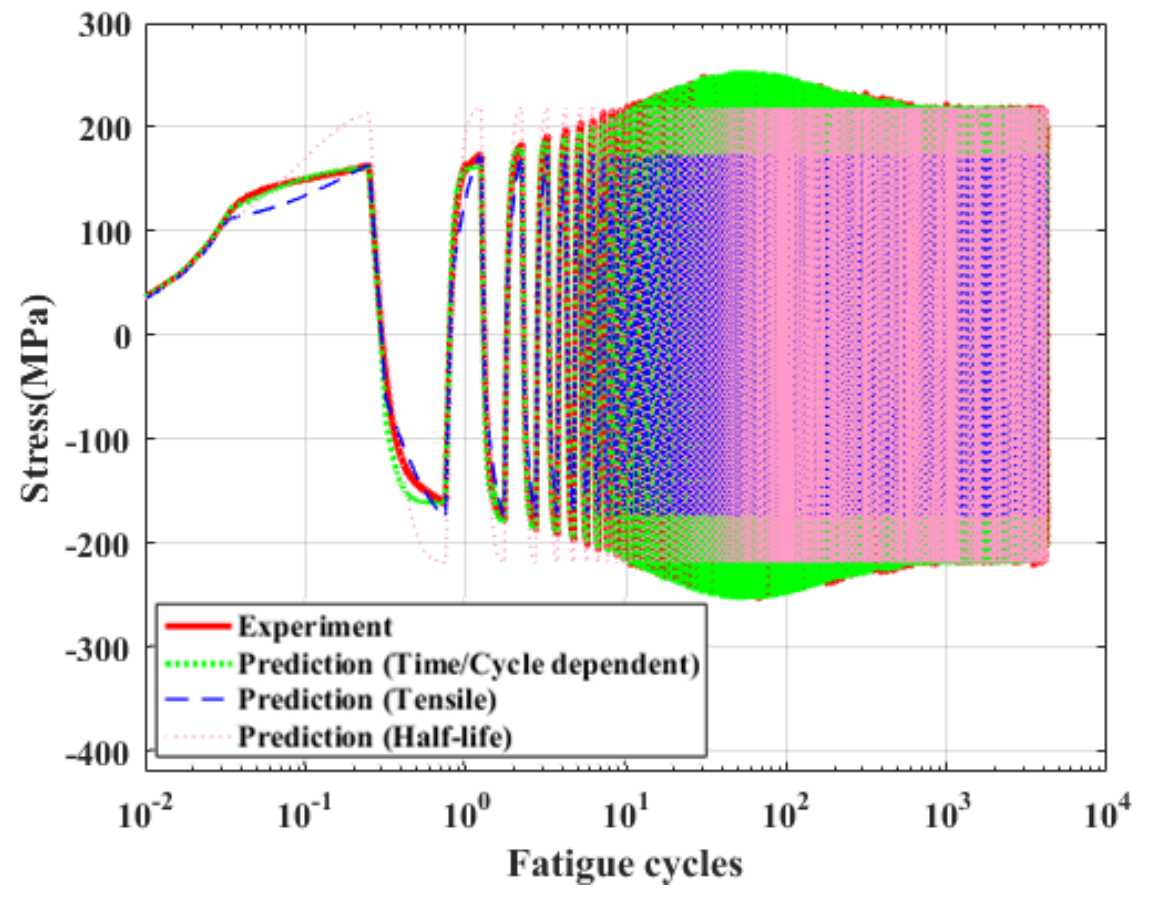

Figure 4. 5 Simulated (analytical) vs experimental axial stress history of ET-F06. Predictions are from simulation using time/cycle-dependent parameters from ET-F06 test and two sets of timeindependent parameters (tensile test ET-T04 and half-life cycle of ET-F06). 


\subsubsection{Variable-Amplitude Fatigue Test}

Time-based analytical modeling of the variable-amplitude fatigue test (ET-F38) was done with time/block-dependent material properties estimated from this test. The ET-F38 test was also analytically modeled using two sets of time-independent or fixed parameters estimated from the tensile test ET-T04 and the half-life block of ET-F38. The experimental and simulated stress is compared in Figure 4.6. Magnified versions of Figure 4.6 are shown in Figures 4.7 to 4.9. These magnified figures represent various stages of material behavior, such as initial hardening, softening and stabilized cycles, and rapid crack propagation and failure, respectively, during the specimen's fatigue life. As seen in the case of constant-amplitude tests (discussed in Section 4.1.1), the evolutionary cycle plasticity model based on time/cycle-dependent material parameters can accurately predict all the stages of material fatigue behavior under variable-amplitude fatigue loading. The experimental and simulated hysteresis stress-strain plots of 12 cycles within the $1^{\text {st }}$ block are shown in Figure 4.10 to demonstrate the importance of the time/blockdependent material parameters. As discussed earlier, the time/block-dependent parameters are amplitude independent and will be used as generic/baseline parameters for predicting the stress-strain state of material subjected to an arbitrary loading profile such as random loading; example results will be discussed in the later part of Section 4.

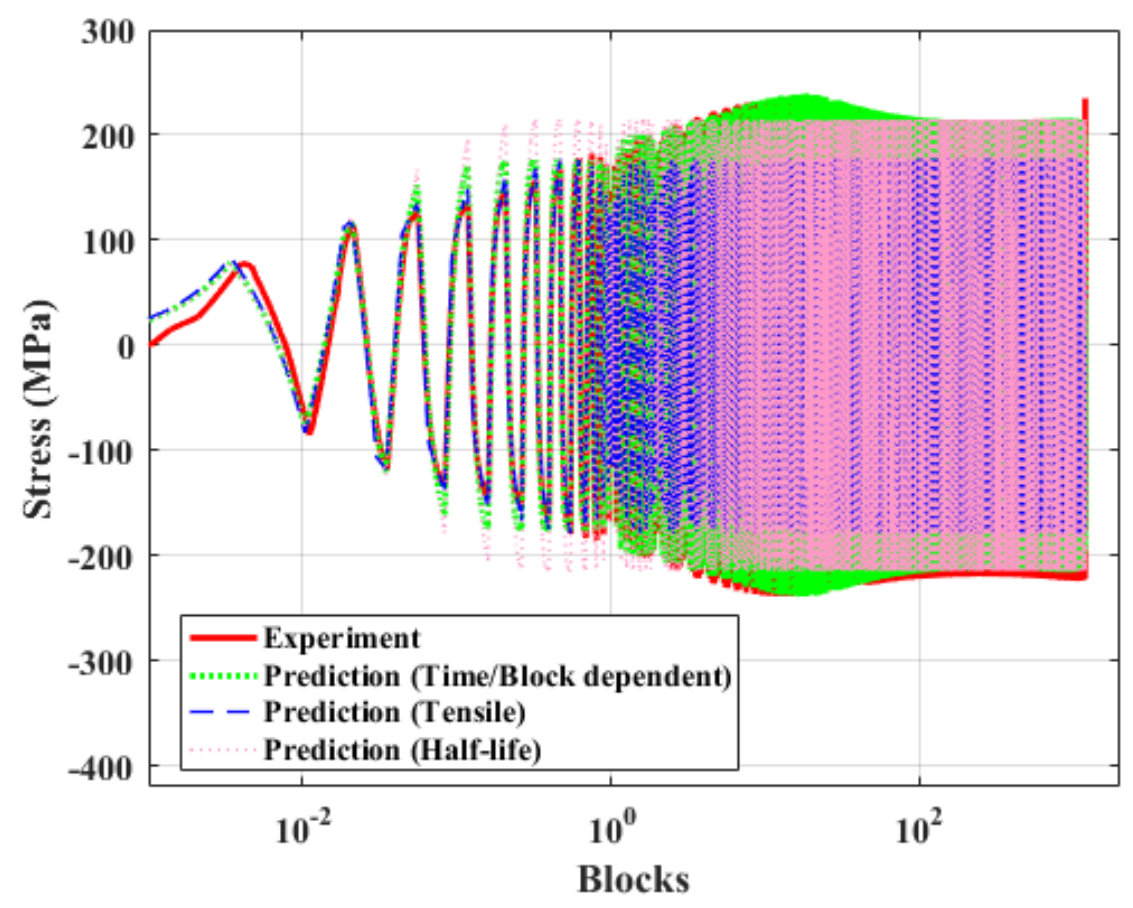

Figure 4. 6 Simulated (analytical) vs experimental axial stress history of ET-F38. Predictions are from simulation using time/block-dependent parameters (ET-F38) and two sets of time-independent parameters (tensile test ET-T04 and half-life block of ET-F38). 


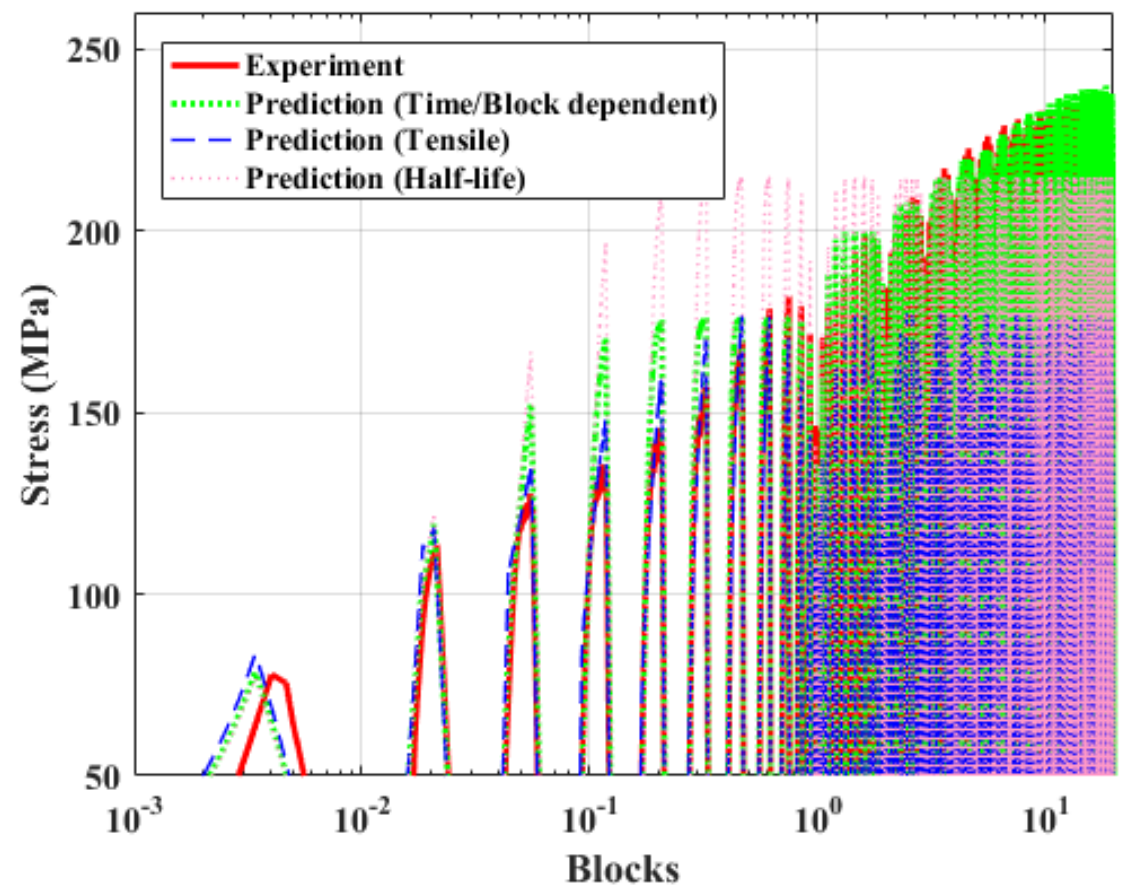

Figure 4. 7 Magnified version of Figure 4.6 showing the ability of evolutionary cyclic plasticity model (time/block-dependent prediction) to predict initial hardening behavior of 316 SS under variableamplitude loading.

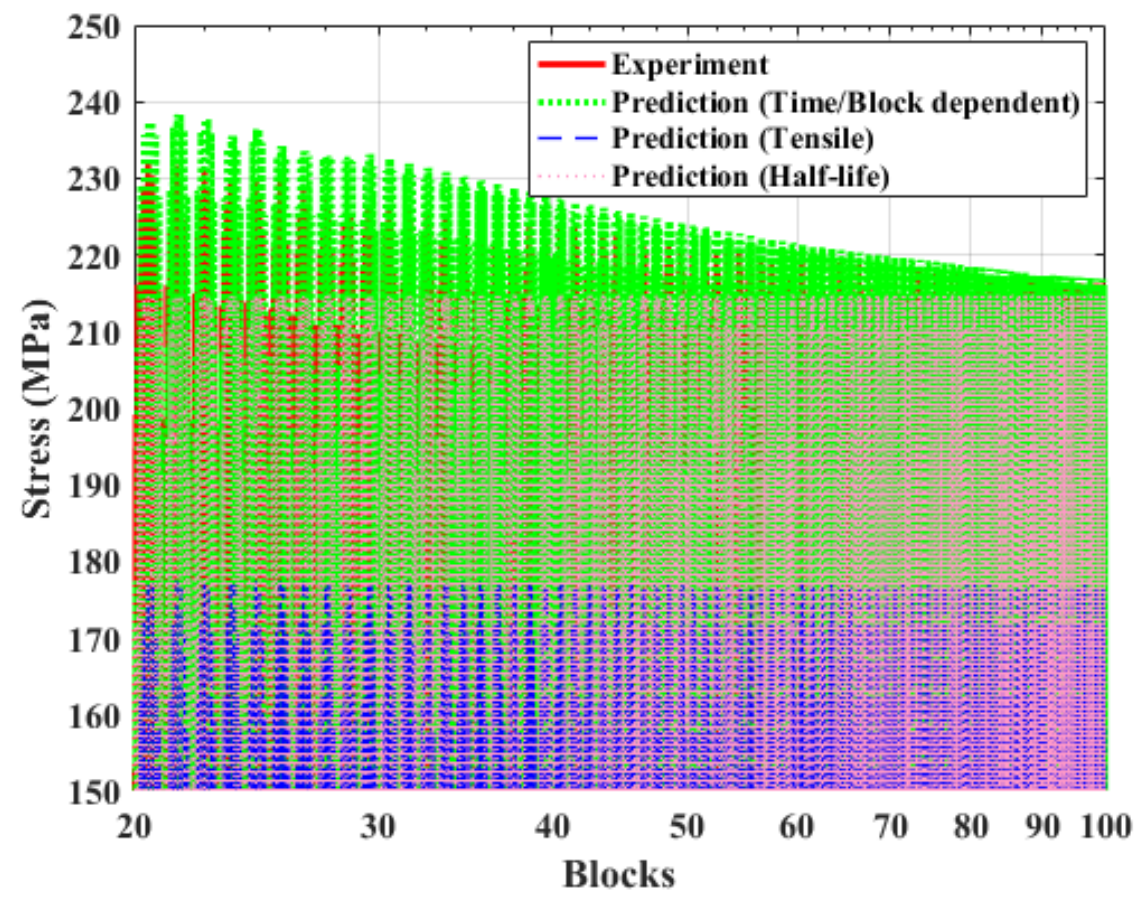

Figure 4. 8 Magnified version of Figure 4.6 showing the ability of evolutionary cyclic plasticity model (time/ block -dependent prediction) to predict softening behavior of 316 SS under variableamplitude loading. 


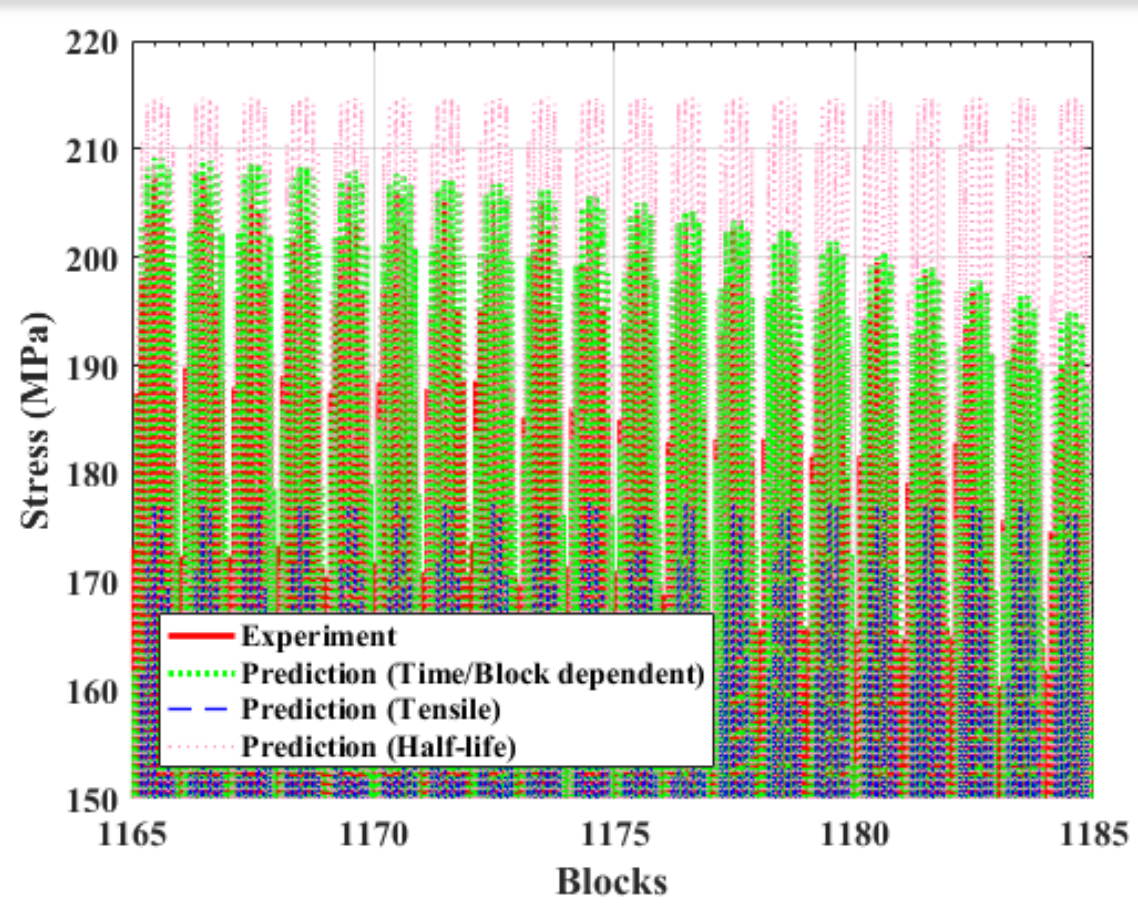

Figure 4. 9 Magnified version of Figure 4.6 showing the ability of evolutionary cyclic plasticity model (time/ block -dependent prediction) to predict the fast stress drop toward the end of the fatigue life of specimen, representing unstable or rapid crack propagation, under variable-amplitude loading.

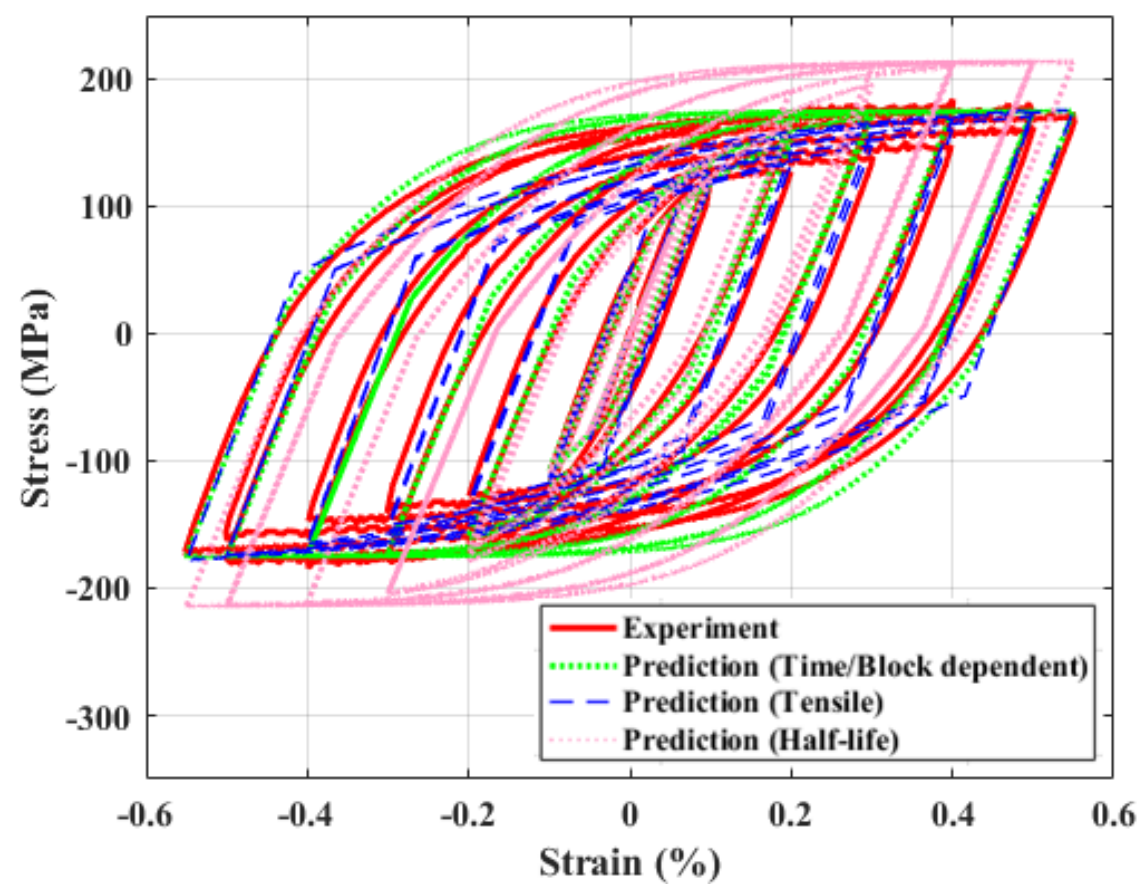

Figure 4. 10 Simulated (analytical) vs experimental hysteresis curves of $1^{\text {st }}$ block (with 12 variableamplitude cycles) of ET-F38. Predictions are from simulation using time/block-dependent parameters and two sets of time-independent parameters (tensile test ET-T04 and half-life block of ET-F38). 


\subsection{Results from Accumulated Plastic Strain Energy (APSE)-Based Analytical Models}

\subsubsection{Constant-Amplitude Fatigue Test}

Constant-amplitude fatigue tests (ET-F41 and ET-F06) were analytically modeled with the evolutionary cyclic plasticity model and APSE-dependent material parameters. The predicted stress profile from the simulation was compared with the experimentally observed stress profiles from both ETF41 and ET-F06. The comparison plots are shown in Figures 4.11 and 4.12 for ET-F41 and ET-F06, respectively. Note that the amplitude condition was the same for both tests, and thus, the predicted (not the experimental/observed) stress profiles in Figures 4.11 and 4.12 are the same. As seen in the figures, the simulated stress profile exhibits all the characteristic behavior (initial hardening followed by softening and stabilized cycles and rapid crack propagation toward the end of fatigue life) of 316 SS under fatigue load. However, the APSE-based prediction is not as close to the experimental data as the time-based prediction. For example, the maximum hardening stress occurs at the $53^{\text {rd }}$ cycle for ET-F06 and ET-F41, while it occurs at the $75^{\text {th }}$ cycle in the simulated results. However, the predicted fatigue life from the simulation was found to be 5860 cycles, which is between experimentally observed fatigue lives for the two experiments (6914 cycles for ET-F41 and 4202 cycles for ET-F06). Section 4.3 provides a comparison between the time- and APSE-based analytical modeling predictions.

For the time-based modeling, the material parameters were estimated from the same test, and therefore, the prediction was very close to the experimental data. By contrast, the material parameters used for APSE-based modeling were estimated from a different test (variable-amplitude test ET-F38), and thus, prediction was not as accurate as for the time-based model. However, considering the variation in experimentally observed fatigue lives between the two constant-amplitude tests with the same conditions, the APSE-based prediction is not bad at all. Moreover, APSE-based modeling provides the robustness of predicting material behavior under any type of amplitude loading, including random. As mentioned earlier, there is a limitation and difficulties in selecting time/cycle/block-dependent parameters for a test case from the parameters estimated from a different fatigue test with different time scale. 


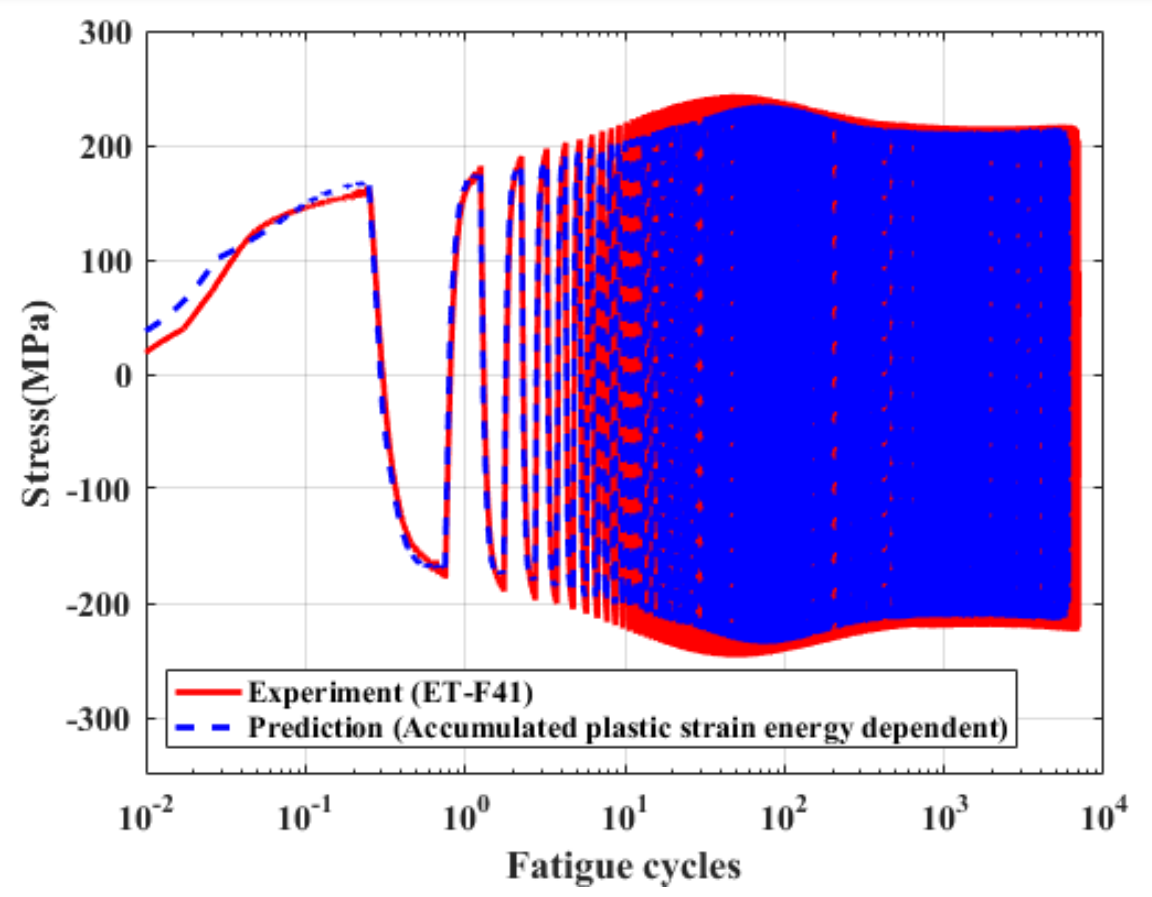

Figure 4. 11 Simulated (analytical) vs experimental axial stress history of ET-F41. Prediction is from simulation using APSE-dependent material parameters estimated from variable-amplitude test (ETF38).

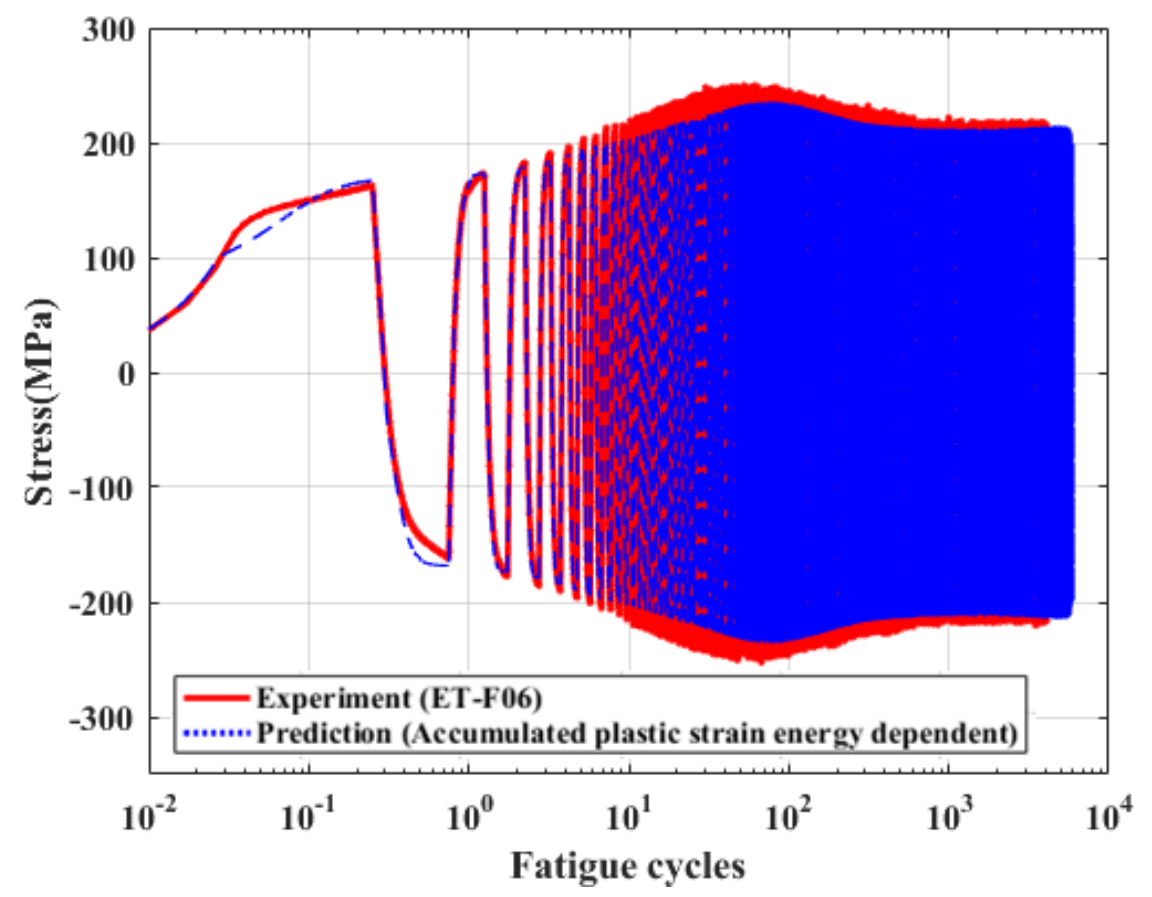

Figure 4. 12 Simulated (analytical) vs. experimental axial stress history of ET-F06. Prediction is from simulation using APSE-dependent material parameters estimated from variable-amplitude test (ETF38). 


\subsubsection{Variable-Amplitude Fatigue Test}

Figure 4.13 compares the experimental stress history observed from the variable-amplitude test ETF38 and the analytically simulated stress using the evolutionary cyclic plasticity model and the APSEdependent material parameters. As seen from the figure, APSE-based analytical modeling can predict the behavior of 316 SS under variable-amplitude loading with good accuracy. The comparison between experimentally observed and predicted life is discussed in Section 4.3.

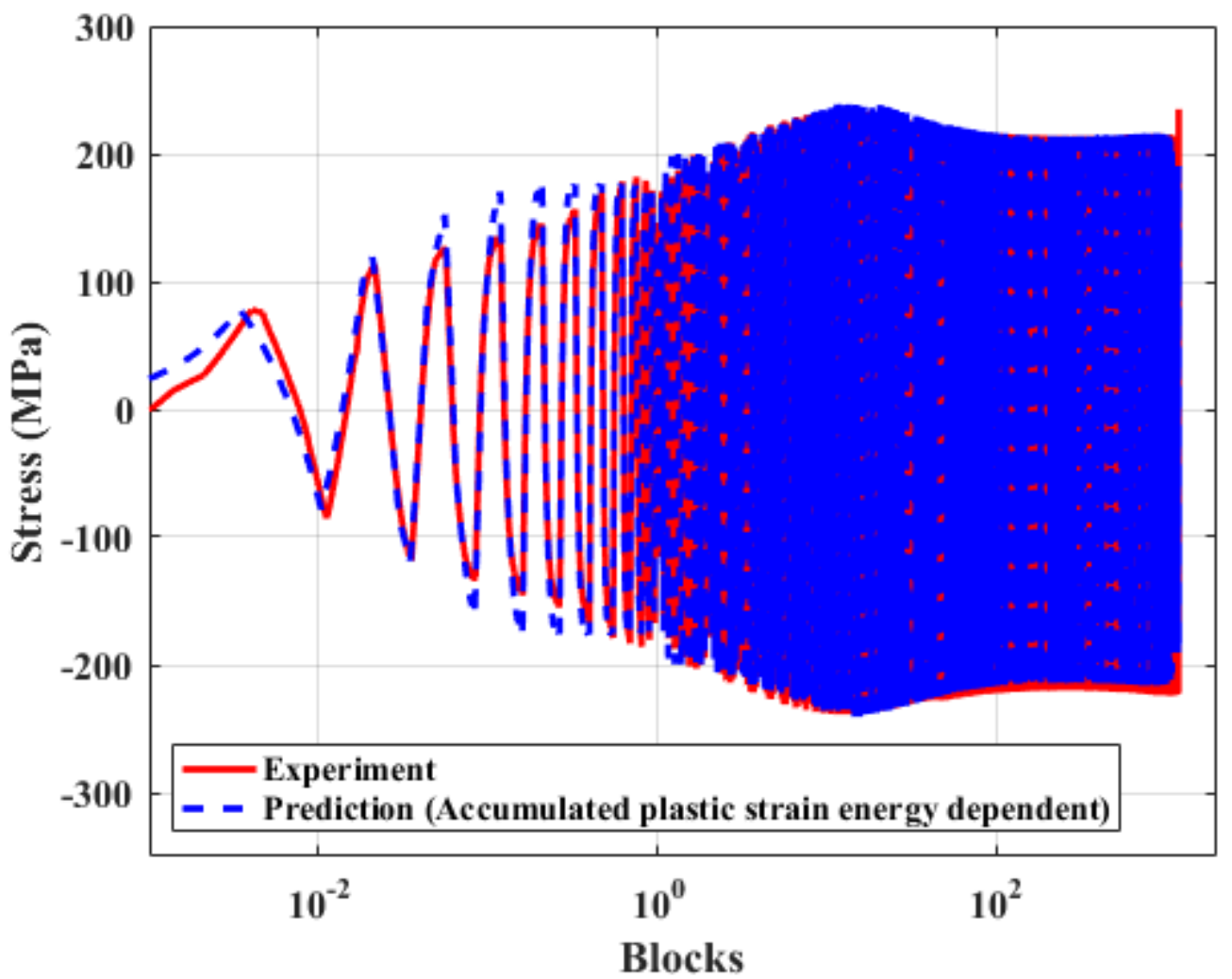

Figure 4.13 Simulated (analytical) vs. experimental axial stress history of ET-F38. Prediction is from simulation using APSE-dependent material parameters estimated from variable-amplitude test (ETF38).

\subsubsection{Random-Amplitude Fatigue Test}

The random-amplitude fatigue test ET-F40 was analytically modeled using the evolutionary cyclic plasticity model and the APSE-dependent material properties estimated from variable-amplitude fatigue test ET-F38. The predicted and experimentally observed stress history is compared in Figure 4.14. Also plotted are results from analytical modeling using fixed material parameters estimated from tensile test ET-T04. The figure shows that the APSE-based evolutionary cyclic plasticity model can accurately predict the stress profile of the material under random-amplitude loading, but that the Chaboche model based on fixed material properties cannot predict the overall hardening and softening behavior. A magnified version 
of Figure 4.14 is shown in Figure 4.15. The experimental and predicted stresses shown in this figure correspond to the strain input shown in Figure 3.24 (see Section 3.4). The corresponding stress-strain hysteresis plots are shown in Figure 4.16. Figures 4.15 and 4.16 depict the complexity in the stress-strain variation during random loading and the great accuracy of the APSE-based modeling in predicting material behavior under this loading. The comparison between the experimentally observed and predicted life is discussed in Section 4.3.

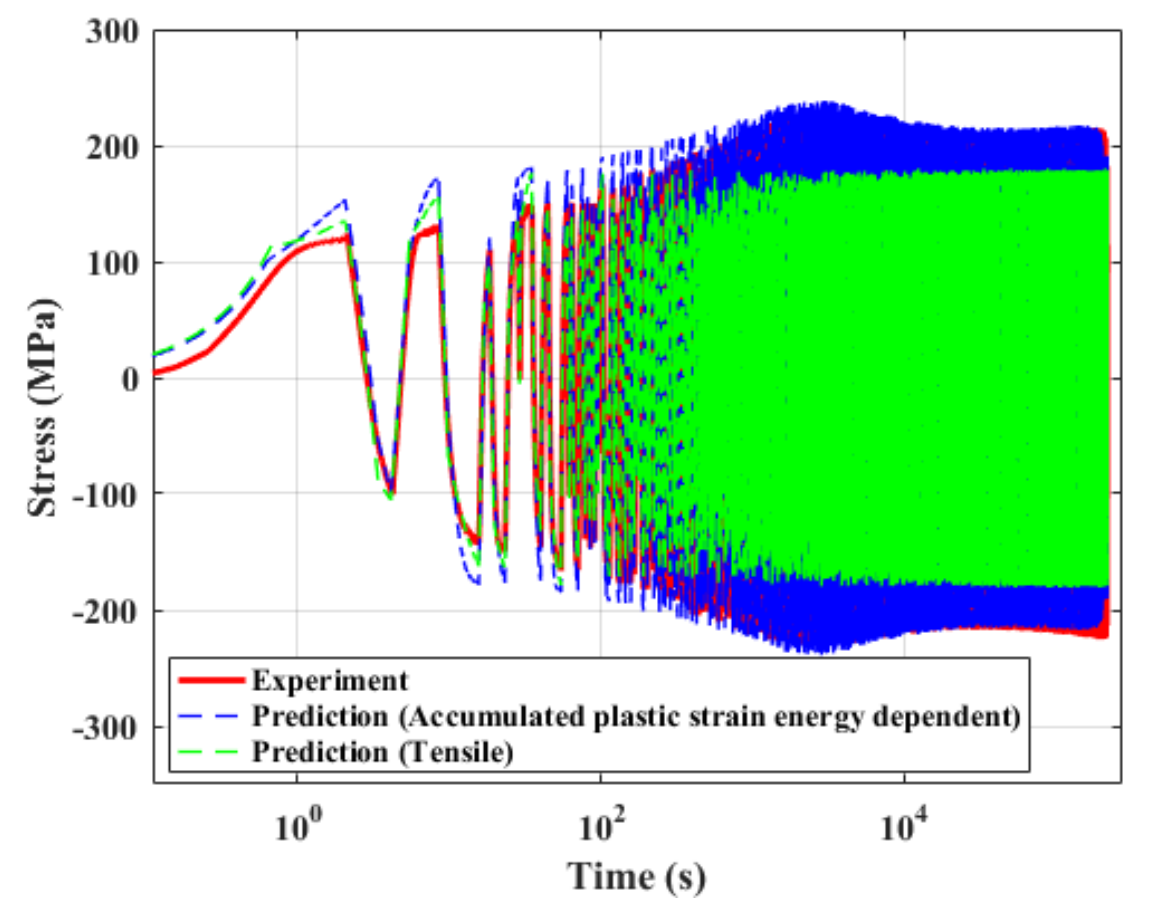

Figure 4.14 Simulated (analytical) vs. experimental axial stress history of ET-F40. Predictions are from simulation using APSE-dependent material parameters estimated from variable-amplitude test (ETF38) and fixed parameters estimated from tensile test (ET-T04). 


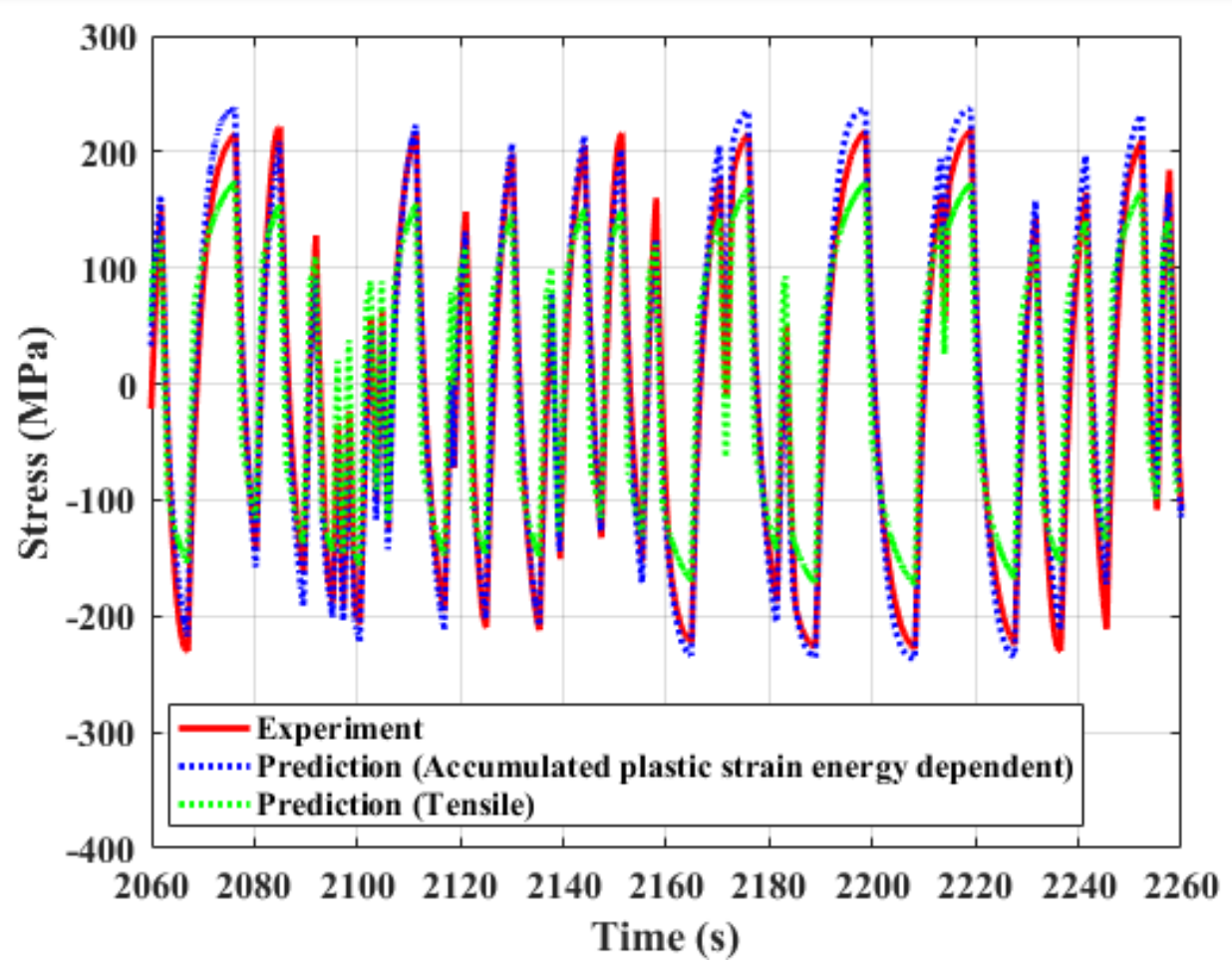

Figure 4.15 Magnified version of Figure 4.14. Experimental and predicted stress corresponds to applied strain shown in Figure 3.24.

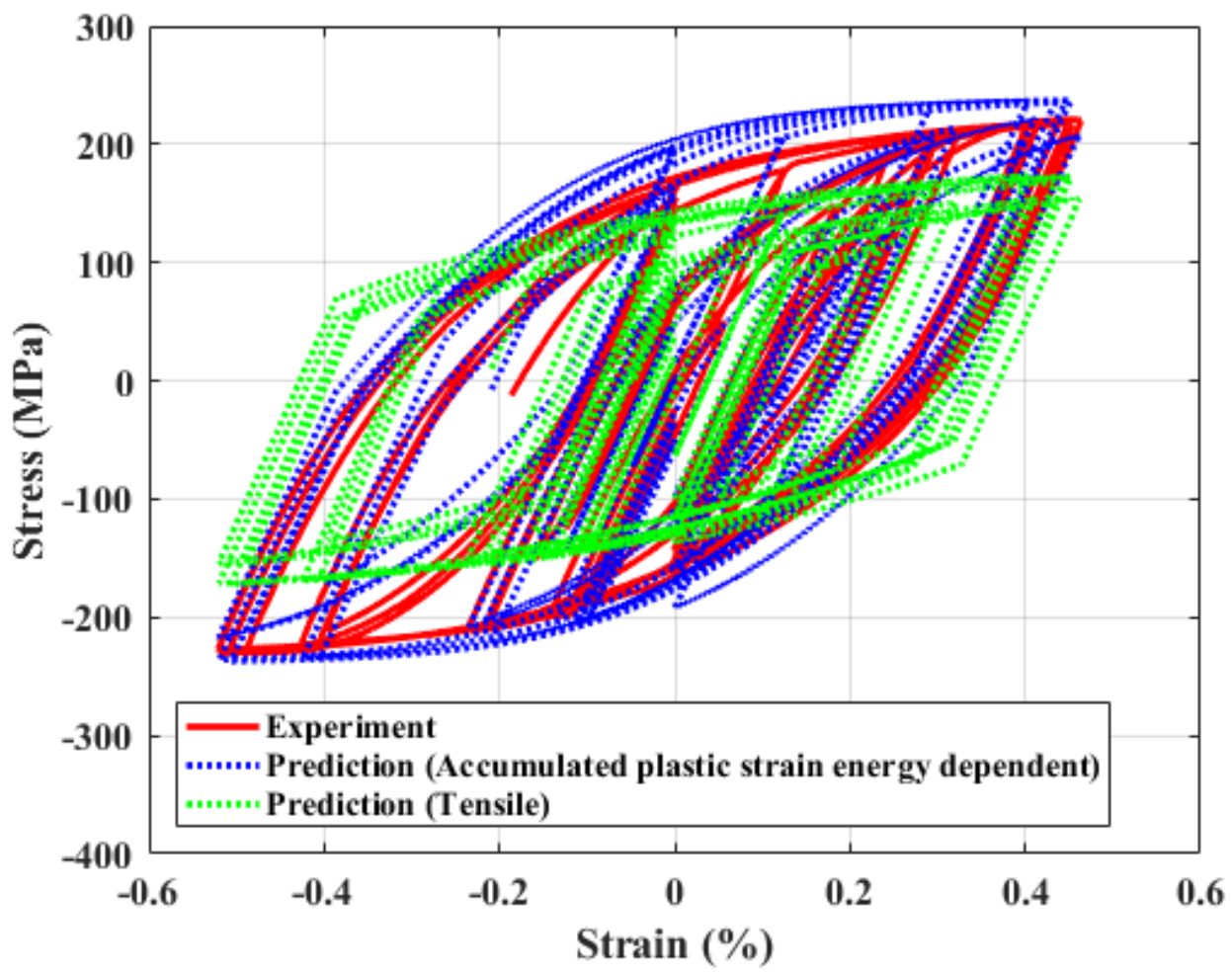

Figure 4.16 Simulated (analytical) vs. experimental hysteresis plot of stress shown in Figure 4.15 and strain shown in Figure 3.24. 


\subsection{Summary of Analytical Modeling Results}

Results from the analytical modeling are summarized here to provide an overall picture of the predicted results and to compare with the experimental results. Table 4.1 compares the experimentally observed maximum hardening stresses during all the fatigue tests with those predicted through time- and APSE-based analytical modeling. As seen from the table, predicted maximum hardening stress is more than $90 \%$ accurate for all the fatigue test cases. However, the time-based prediction is more accurate in general, as the material parameters used for modeling were estimated from the same test.

Table 4. 1 Experimentally observed and predicted (analytical modeling) maximum hardening stress.

\begin{tabular}{|c|c|c|c|c|c|c|}
\hline \multirow{2}{*}{$\begin{array}{c}\text { Test } \\
\text { ID }\end{array}$} & \multirow{2}{*}{$\begin{array}{c}\text { Amplitude } \\
\text { type }\end{array}$} & $\begin{array}{c}\text { Experimental } \\
\text { observation }\end{array}$ & \multicolumn{4}{|c|}{ Analytical Modeling } \\
\cline { 3 - 7 } & & Stress (MPa) & $\begin{array}{c}\text { Time-based prediction } \\
\text { (MPa) }\end{array}$ & $\begin{array}{c}\text { Accuracy } \\
(\%)\end{array}$ & $\begin{array}{c}\text { Stress } \\
\text { (MPa) }\end{array}$ & $\begin{array}{c}\text { Accuracy } \\
(\%)\end{array}$ \\
\hline F06 & Constant & 250.0 & 254.5 & 98.2 & & \multirow{2}{*}{$95.2^{\#}$} \\
\hline F41 & Constant & 245.5 & 252.2 & 97.3 & & \\
\hline F38 & Variable & 234.9 & 238.9 & 98.3 & 240.4 & 97.7 \\
\hline F40 & Random & 223.5 & N/A & N/A & 238.3 & 93.4 \\
\hline
\end{tabular}

\# An average experimental maximum hardening stress (247.8 MPa) based on observation from ET-F06 and ET-F41 tests is used to calculate the accuracy in predicted maximum hardening stress.

* Time-based modeling is not possible for random-amplitude loading.

To determine the fatigue lives of the specimen, a failure criterion is required. As all the tests were performed uniaxially and under strain control, a drop in uniaxial stress in the direction of applied strain was used for determining a failure criterion for the fatigue tests. Conventionally, a $25 \%$ load-drop from the maximum load is used as a failure criterion of fatigue specimens. The experimentally observed maximum stress and failure stress values according to the $25 \%$ load drop criterion are given in Table 4.2. The table also provides experimental observations during fatigue testing. As mentioned in Table 4.2, the ET-F06 and ET-F38 specimens broke into two pieces before the stress dropped to 25\% load-drop stress. Furthermore, the ET-F06 specimen broke as early as the stress dropped to $199.5 \mathrm{MPa}$. Thus, a stress drop to $200 \mathrm{MPa}$ was used as failure criterion for all the strain-controlled fatigue tests. 
Table 4. 2 Determination of failure criteria for simulation results.

\begin{tabular}{|c|c|c|c|c|c|}
\hline $\begin{array}{c}\text { Test } \\
\text { ID }\end{array}$ & $\begin{array}{c}\text { Amplitude } \\
\text { type }\end{array}$ & $\begin{array}{l}\text { Experimental } \\
\text { maximum } \\
\text { stress }(\mathrm{MPa})\end{array}$ & $\begin{array}{c}\text { Failure stress } \\
\text { (MPa) } \\
\text { according to } \\
25 \% \text { load-drop } \\
\text { criteria }\end{array}$ & Experimental observation & $\begin{array}{l}\text { Stress value } \\
\text { used as } \\
\text { failure } \\
\text { criteria }\end{array}$ \\
\hline F06 & Constant & 250 & 187.5 & $\begin{array}{l}\text { Stress at breaking point: } 199.5 \mathrm{MPa} \\
\quad \text { (specimen broke into } 2 \text { pieces) }\end{array}$ & \multirow{4}{*}{$200 \mathrm{MPa}$} \\
\hline $\mathrm{F} 41$ & Constant & 245.5 & 184.1 & $\begin{array}{c}\text { Stress continued to drop below } \\
\text { 184.1 MPa without breaking into } 2 \\
\text { pieces }\end{array}$ & \\
\hline F38 & Variable & 234.9 & 176.2 & $\begin{array}{l}\text { Stress at breaking point: } 175.2 \mathrm{MPa} \\
\quad \text { (specimen broke into } 2 \text { pieces) }\end{array}$ & \\
\hline $\mathrm{F} 40$ & Random & 223.5 & 167.6 & $\begin{array}{c}\text { Stress continued to drop below } \\
\text { 167.6 MPa without breaking into } 2 \\
\text { pieces }\end{array}$ & \\
\hline
\end{tabular}

The experimental and predicted fatigue lives for all the fatigue tests and analytical modeling cases are provided in Table 4.3. As seen from the table, the Chaboche model based on time-independent or fixed material parameters predicts infinite fatigue life for all cases. The evolutionary cyclic plasticity model predicts the fatigue life of the specimen with an accuracy of more than $90 \%$. The accuracy in predicted life based on time-dependent material parameters is almost $100 \%$ for both constant- and variableamplitude fatigue tests as the material parameters were estimated from same test. Because the time-based approach cannot be used for predicting material behavior under random-amplitude loading, the APSEbased approach was used. The predicted fatigue life for the random-amplitude fatigue test was surprisingly accurate $(94.7 \%)$. 
Table 4. 3 Experimental and predicted (analytical modeling) fatigue lives.

\begin{tabular}{|c|c|c|c|c|c|c|c|c|}
\hline \multirow{3}{*}{$\begin{array}{c}\text { Test } \\
\text { ID }\end{array}$} & \multirow{3}{*}{$\begin{array}{c}\text { Amplitude } \\
\text { type }\end{array}$} & \multirow{3}{*}{$\begin{array}{l}\text { Fatigue } \\
\text { life unit }\end{array}$} & \multirow{3}{*}{$\begin{array}{c}\text { Experiment } \\
\text { Observed } \\
\text { life } \\
\end{array}$} & \multicolumn{5}{|c|}{ Analytical Modeling } \\
\hline & & & & \multirow{2}{*}{$\begin{array}{c}\text { Fixed } \\
\text { properties } \\
\text { (tensile/half- } \\
\text { life) }\end{array}$} & \multicolumn{2}{|c|}{ Time-based } & \multicolumn{2}{|c|}{ APSE-based } \\
\hline & & & & & $\begin{array}{c}\text { Predicted } \\
\text { Life }\end{array}$ & $\begin{array}{c}\text { Accuracy } \\
(\%)\end{array}$ & $\begin{array}{l}\text { Predicted } \\
\text { life }\end{array}$ & $\begin{array}{c}\text { Accuracy } \\
(\%)\end{array}$ \\
\hline $\begin{array}{l}\text { ET- } \\
\text { F06 }\end{array}$ & Constant & Cycles & 4202 & $\infty$ & 4200 & 100.0 & \multirow{2}{*}{5860} & \multirow{2}{*}{$94.6^{\#}$} \\
\hline $\begin{array}{l}\text { ET- } \\
\text { F41 }\end{array}$ & Constant & Cycles & 6914 & $\infty$ & 6932 & 99.7 & & \\
\hline $\begin{array}{l}\text { ET- } \\
\text { F38 }\end{array}$ & Variable & Blocks & 1179 & $\infty$ & 1180 & 99.9 & 1090 & 92.5 \\
\hline $\begin{array}{l}\text { ET- } \\
\text { F40 }\end{array}$ & Random & Time (s) & 21590 & $\infty$ & $\mathrm{N} / \mathrm{A}^{*}$ & $\mathrm{~N} / \mathrm{A}^{*}$ & 20440 & 94.7 \\
\hline
\end{tabular}

\# An average experimental life (5558 cycles) based on observation from ET-F06 and ET-F41 tests is used to calculate the accuracy in predicted life.

* Time-based modeling is not possible for random amplitude loading 


\section{Results from FE Modeling}

One of our major tasks in mechanics-based fatigue modeling is to develop an FE model framework based on the previously discussed evolutionary cyclic plasticity model. The FE model framework can then be used for extrapolating material behavior based on uni-axial tests to a multi-axial domain for stress analysis and fatigue evaluation of realistic reactor components, which are ideally subjected to multi-axial loading. Compared to the conventional FE model, the evolutionary cyclic plasticity FE model would be able to predict the cyclic hardening and softening behavior of a component. It is assumed that, similar to the conventional tensile-test-based FE model, which is extensively used by industry for stress analysis of metallic components subjected to monotonic loading transients, the new FE model would be able to simulate a component subjected to cyclic loading. Also similar to the tensile-test-based FE model, it is assumed that the translation from a uni-axial to multi-axial fatigue-test-based model is isotropic and based on the assumption that the metallic components are homogeneous, with material behavior being similar in all directions. However, before the new FE model can be used for component-level stress analysis, the FE framework must be validated with experimental test cases.

We used a commercially available FE software, ABAQUS, for FE implementation of the proposed evolutionary cyclic plasticity model. The FE implementation technique is discussed in Section 2, and results from time- and APSE-based FE simulations are presented here. A single 3D 8-node brick element representing the gauge section $(0.5 \mathrm{in}$.) was used for $\mathrm{FE}$ simulation of the fatigue experiments. A single element was used to reduce the computational time for simulating thousands of fatigue cycles. However, we assume that if the basic modeling technology is available, it would not be difficult to model a real-life component in an age of exascale supercomputing. Nevertheless, the first priority in this work is to validate the proposed evolutionary plasticity FE model by the 3D-FE modeling of the earlier discussed fatigue test specimens. In the new FE model, the cross-section of the 3D brick element (hexahedral: 8-node linear brick element: C3D8) was considered equal to the nominal cross-section of the specimen.

The geometry of the fatigue specimen and the 3D ABAQUS model are shown in Figures 5.1 and 5.2, respectively. Simulations representing strain-controlled fatigue tests with constant, variable, and random amplitudes were performed by applying corresponding deformation in the z-direction, as shown by the arrows in Figure 5.2. An example simulated axial-stress profile at a given instant for a general fatigue test simulation is shown Figure 5.3. 


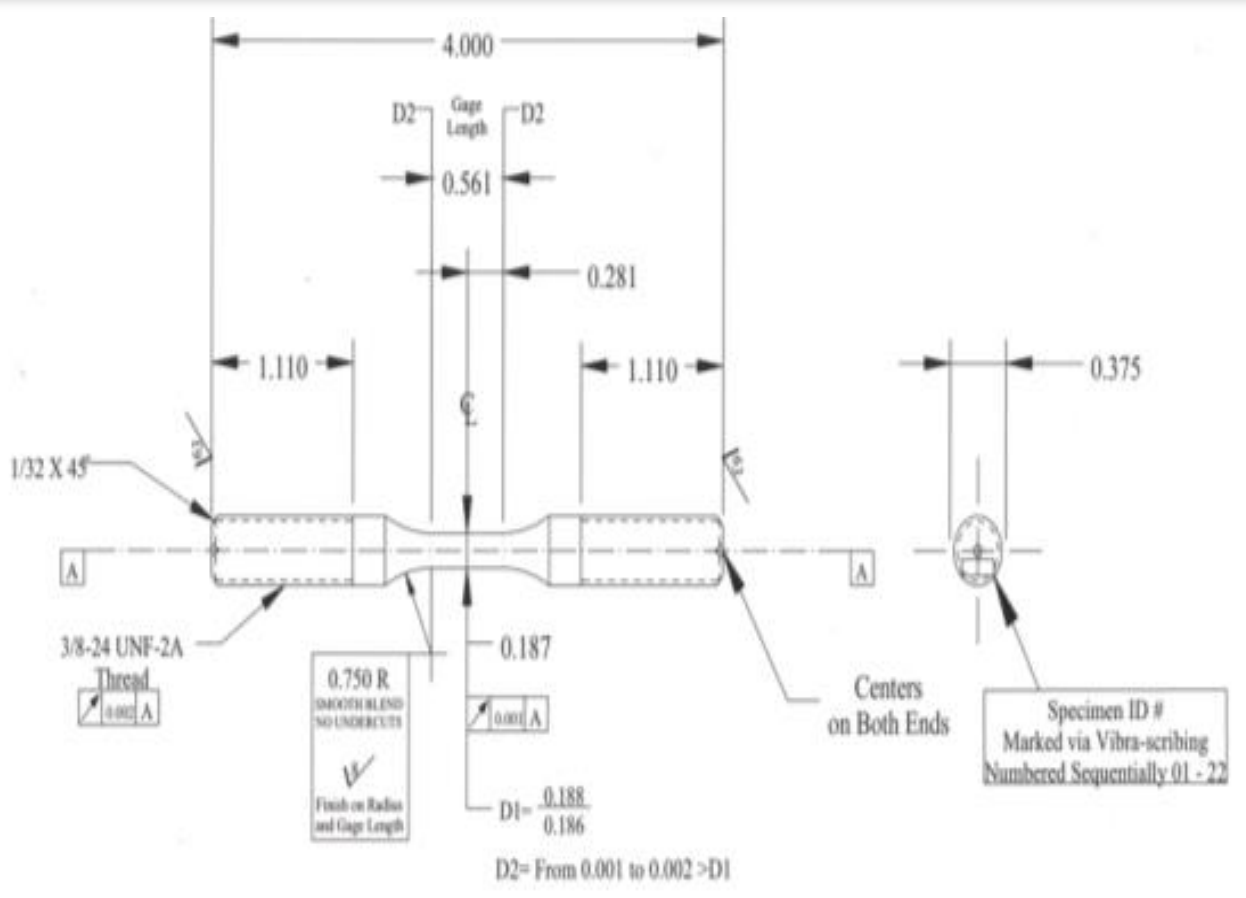

Figure 5. 1 Geometry of fatigue test specimen.

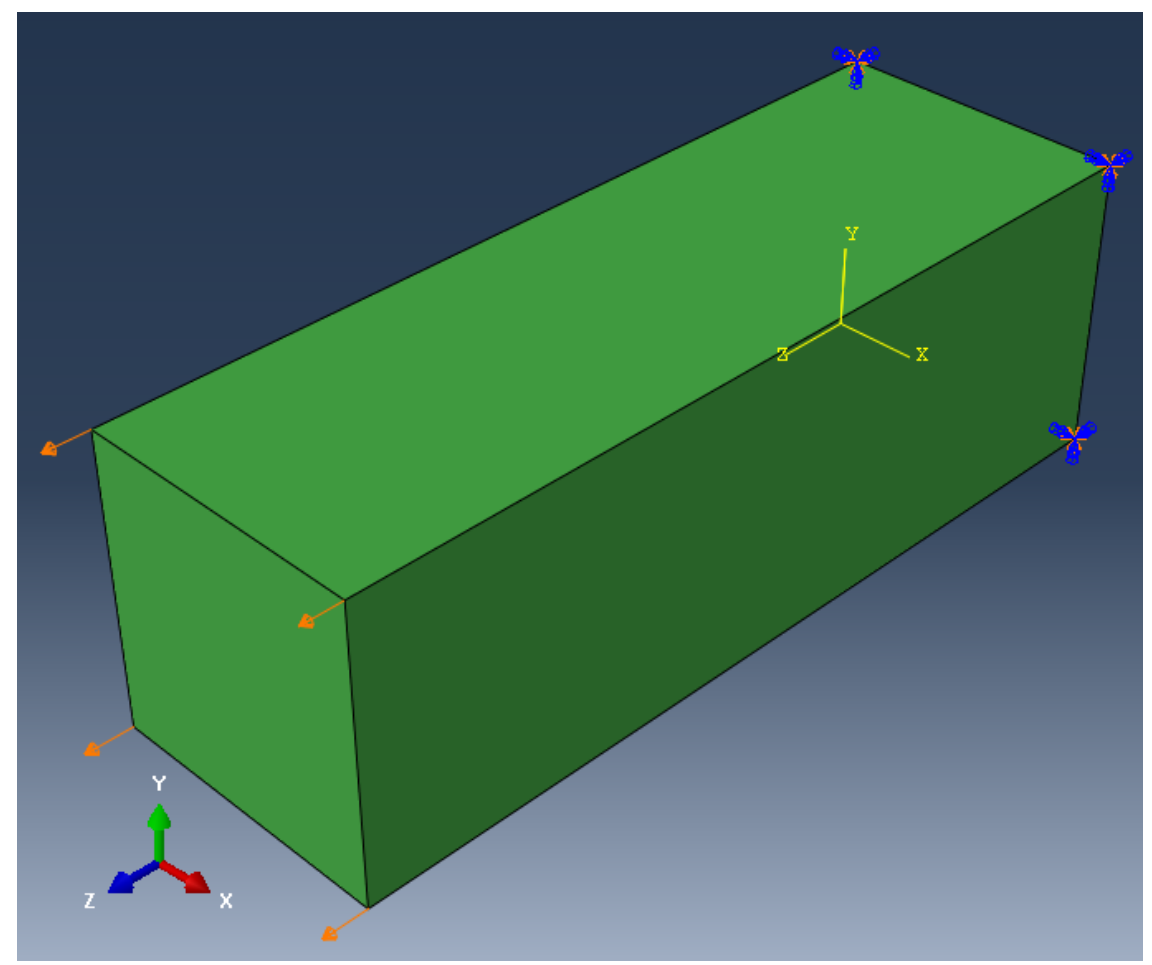

Figure 5.2 Three-dimensional 8-node brick element used for FE simulation of fatigue experiment in ABAQUS. 


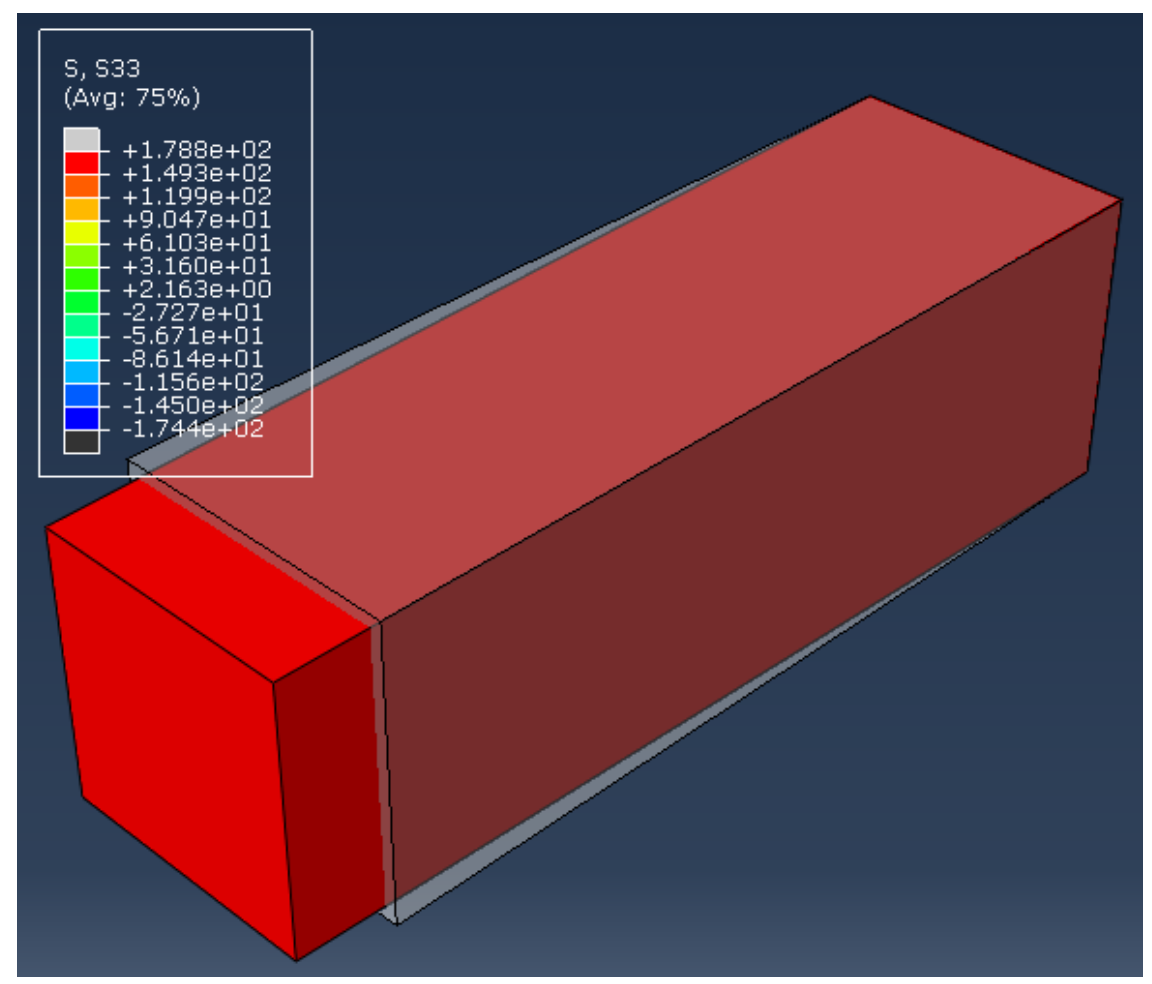

Figure 5.3 An example simulated stress profile at a given instant during fatigue test simulation.

\subsection{Results from Time-Based 3D-FE Models}

\subsubsection{Constant-Amplitude Fatigue Test}

We performed three 3D-FE simulations representing the ET-F41 fatigue experiment using time/cycledependent and time-independent material properties. Figure 5.4 compares the experimentally observed and simulated stress for the first 100 cycles. The figure shows predicted stress using time/cycle-dependent material parameters estimated from ET-F41 and two sets of time-independent or fixed parameters estimated from tensile test ET-T04 and the half-life cycle of ET-F41. A magnified version of Figure 5.4 is shown in Figure 5.5. It can be seen from the figures that the evolutionary cyclic plasticity model based on time/cycle-dependent material properties can accurately predict the material hardening behavior, while the model based on time-independent material properties estimated from ET-T04 and half-life cycle of ET-F41 could not predict the material hardening behavior. The ABAQUS model was then simulated for the entire life of the fatigue specimen in the FE model framework. The 3D-FE simulated axial stress along with the experimentally observed stress for the entire life of the specimen is shown in Figure 5.6. A magnified version of Figure 5.6, demonstrating initial stress hardening and then softening followed by stabilized cycles, is shown in Figure 5.7. These figures demonstrate that the evolutionary cyclic plasticity model predicts not only the stress hardening but also the stress softening with significant accuracy. The model also predicts the stabilized cycles, which represent a quasi-stable state during fatigue. Most 
importantly, as shown in Figure 5.8, it accurately predicts the fast stress drop toward the end of the fatigue life of the specimen, which represents unstable or rapid crack propagation.

Results from the 3D-FE simulation of the constant-amplitude fatigue test ET-F06 are shown in Figure 5.9. As seen in the case of ET-F41, the evolutionary cyclic plasticity FE model using time/cycle-dependent material properties can accurately predict the material behavior during ET-F06.

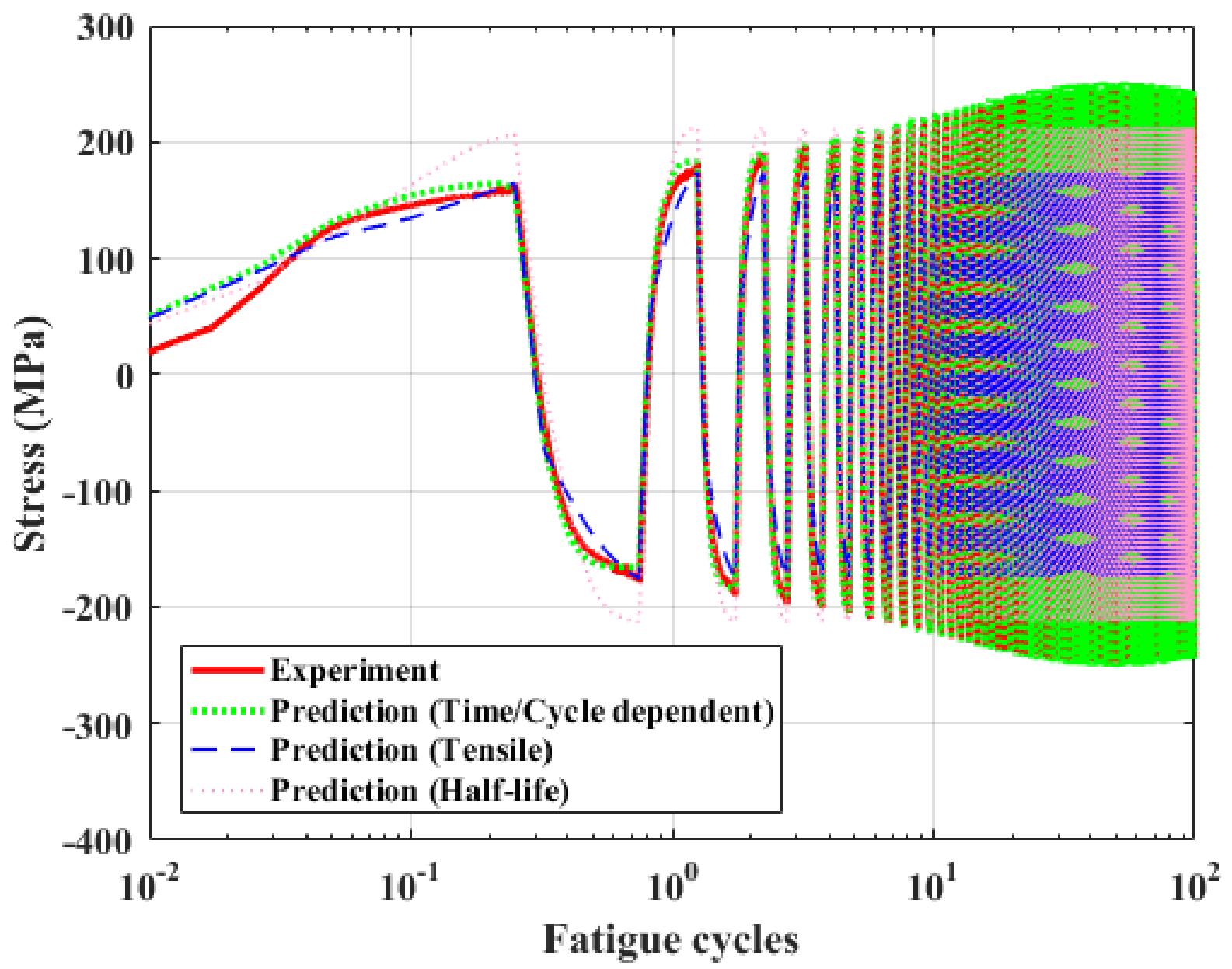

Figure 5.4 3D-FE simulated (evolutionary cycle plasticity model) vs. experimental axial stress of ET-F41 specimen for first 100 cycles. Predictions are from simulation using time/cycle-dependent parameters (ET-F41) and two sets of time-independent parameters (tensile test ET-T04 and half-life cycle of ET-F41). 


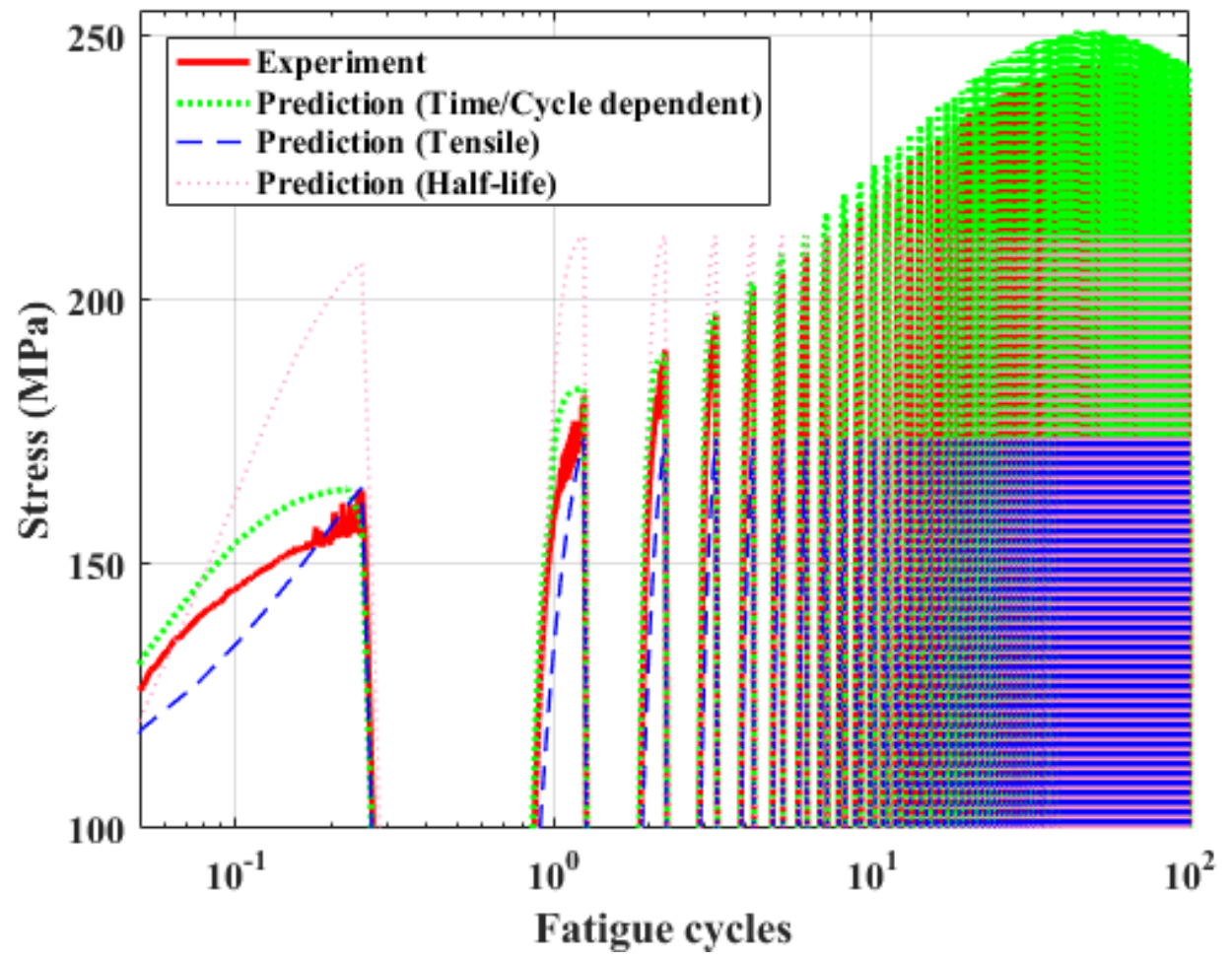

Figure 5.5 Magnified version of Figure 5.4.

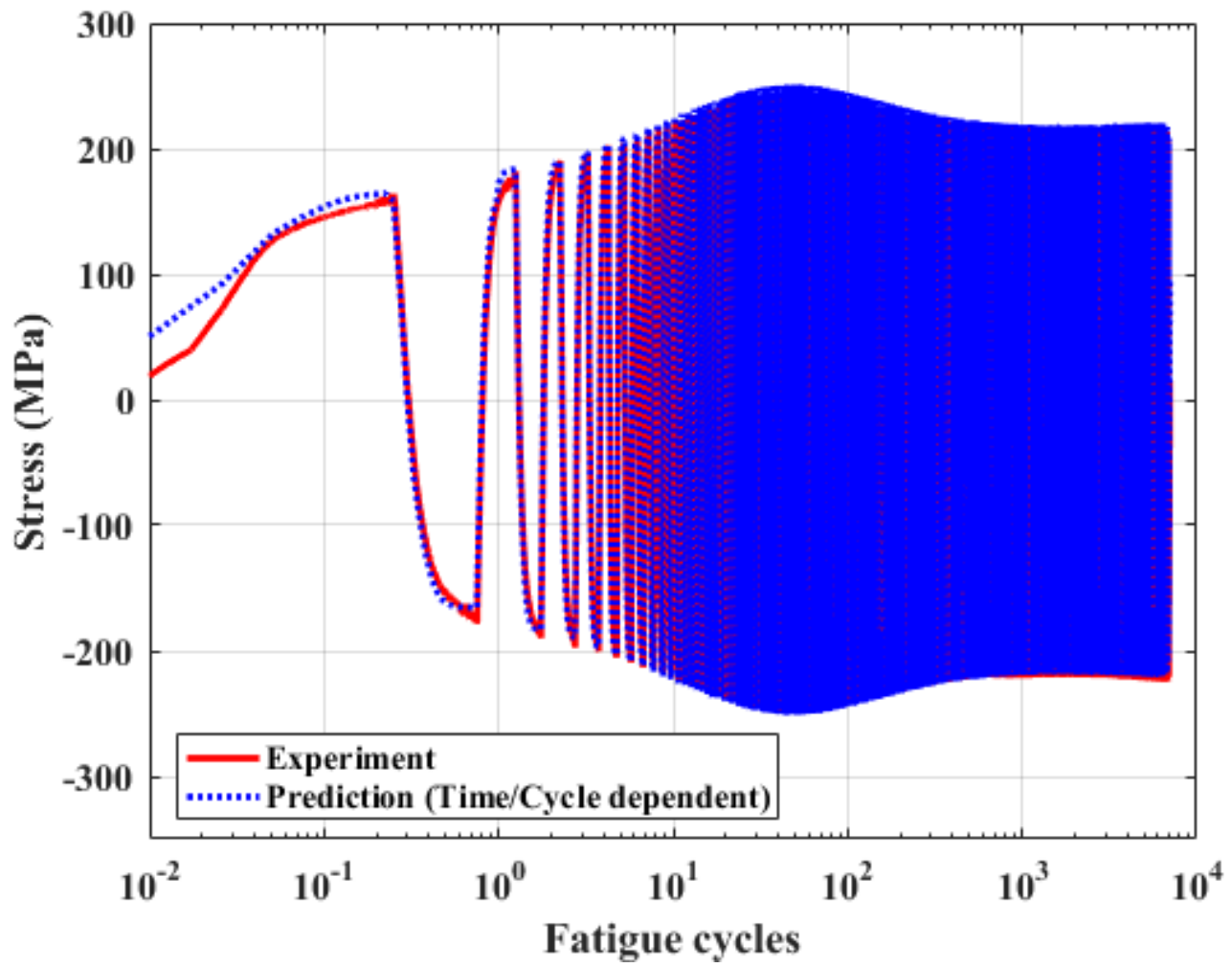

Figure 5.6 3D-FE simulated (evolutionary cycle plasticity model) vs. experimental axial stress of ET-F41 specimen for whole fatigue life. 


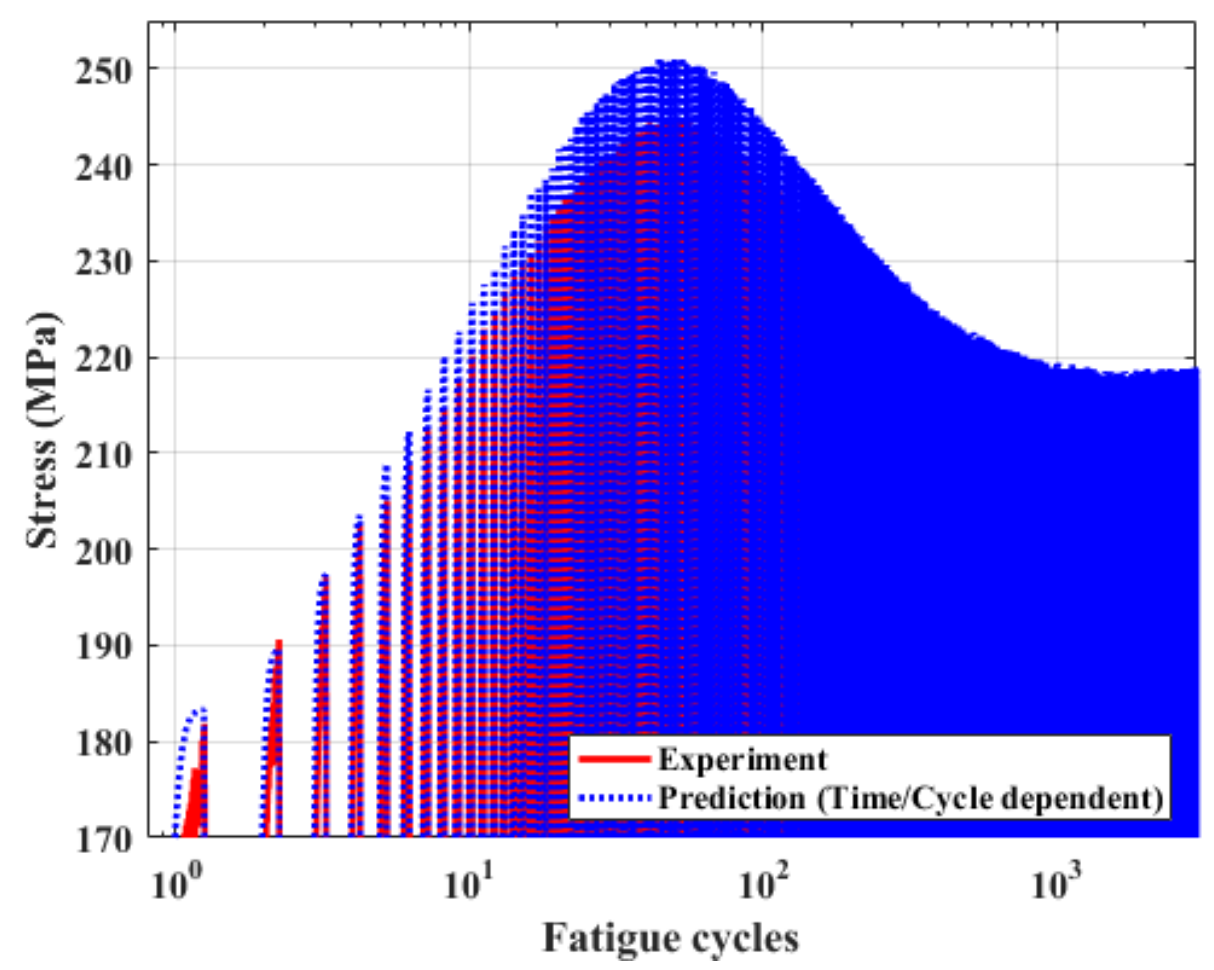

Figure 5.7 Magnified version of Figure 5.6 showing that the 3D-FE results can predict material behavior during initial stress hardening, softening, and stabilized cycles under constant-amplitude loading.

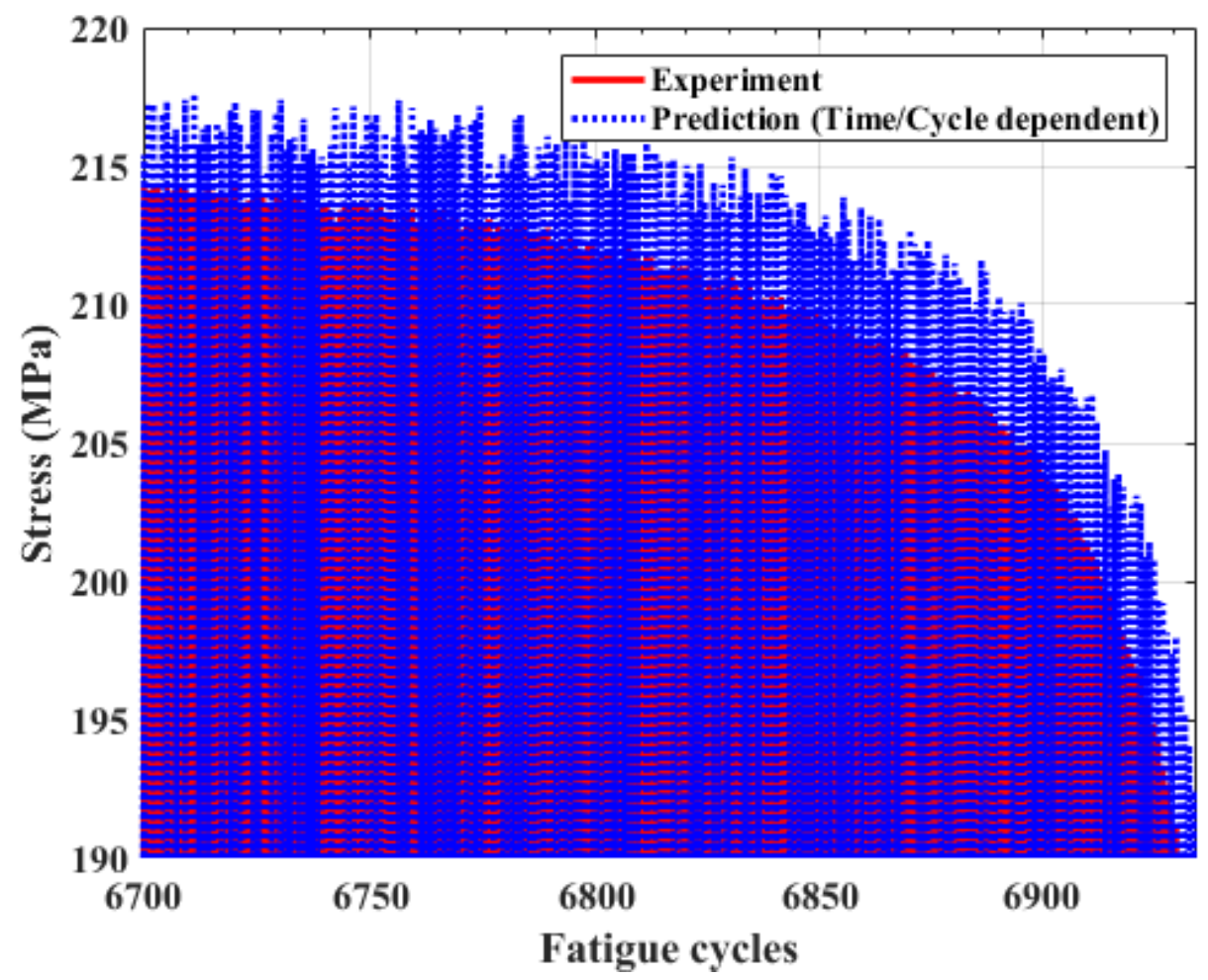

Figure 5.8 Magnified version of Figure 5.6 showing that the 3D-FE results can predict material behavior during rapid crack propagation and failure under constant-amplitude loading. 


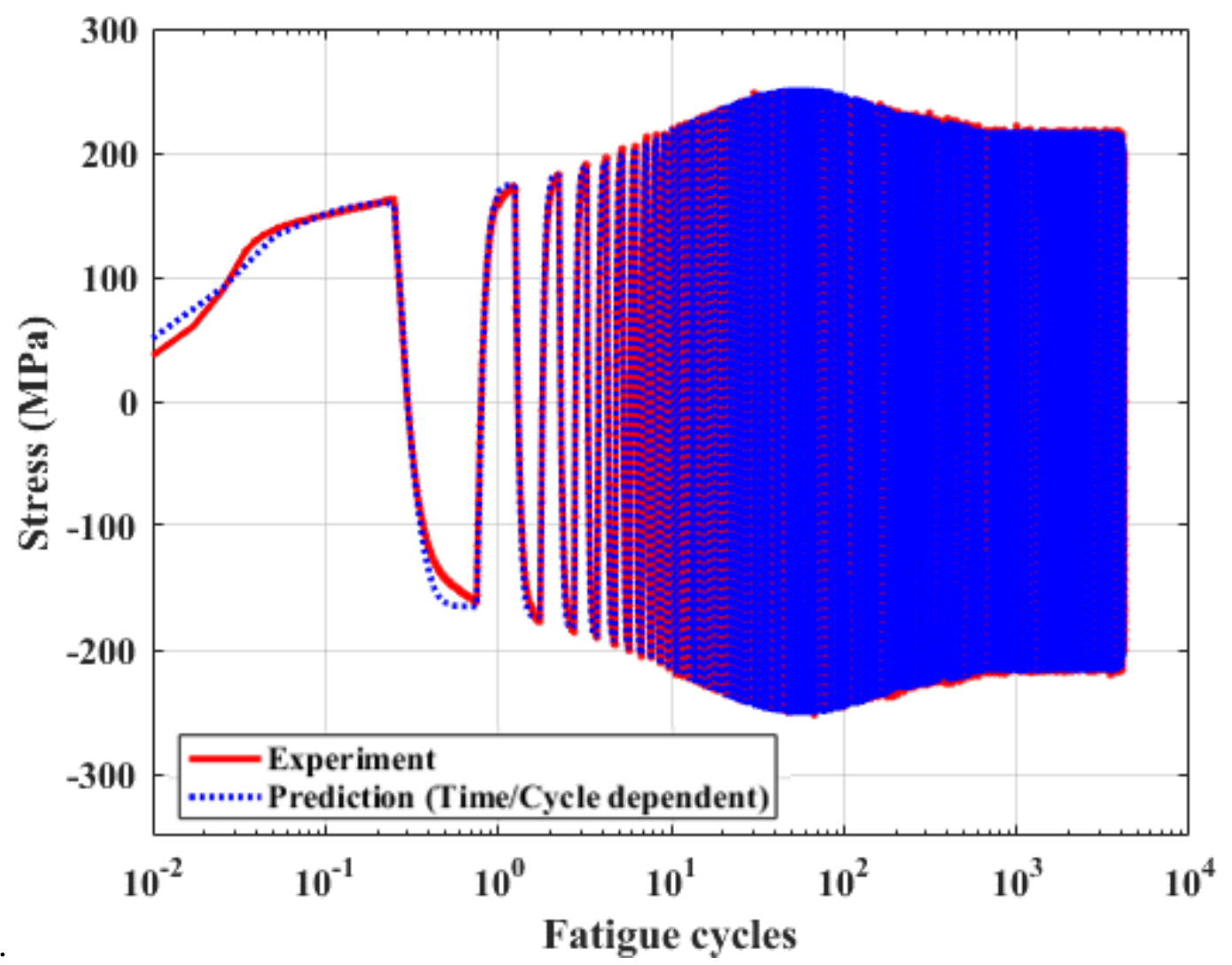

Figure 5.9 3D-FE simulated (evolutionary cycle plasticity model) vs. experimental axial stress of ET-F06 specimen for whole fatigue life.

\subsubsection{Variable-Amplitude Fatigue Test}

We performed 3D-FE simulations of variable-amplitude fatigue test ET-F38 using time/blockdependent material properties and time-independent material properties. The simulated stress profiles (first 50 blocks) are compared with the experimentally observed stresses in Figure 5.10. As seen in the case of constant-amplitude fatigue tests, the evolutionary cyclic plasticity model can accurately predict the material behavior under variable-amplitude loading using the block-dependent material properties. By contrast, the FE model using time-independent fixed parameters does not predict the material behavior well. The entire fatigue life of the ET-F38 specimen was simulated using the block-dependent material properties. The 3D-FE simulated axial stress along with experimentally observed stress for the entire fatigue life of ET-F38 specimen is shown in Figure 5.11. Different magnified versions of Figure 5.11 are also shown in Figures 5.12 and 5.13.

As seen from Figures 5.12 and 5.13, the 3D-FE simulated stress profile exhibits all the characteristic fatigue life stages (initial hardening, softening, stabilized cycles, and rapid crack propagation followed by failure) and is close to the experimentally observed stresses. The experimental and FE simulated maximum hardening stresses and fatigue lives are compared in Section 5.3. 


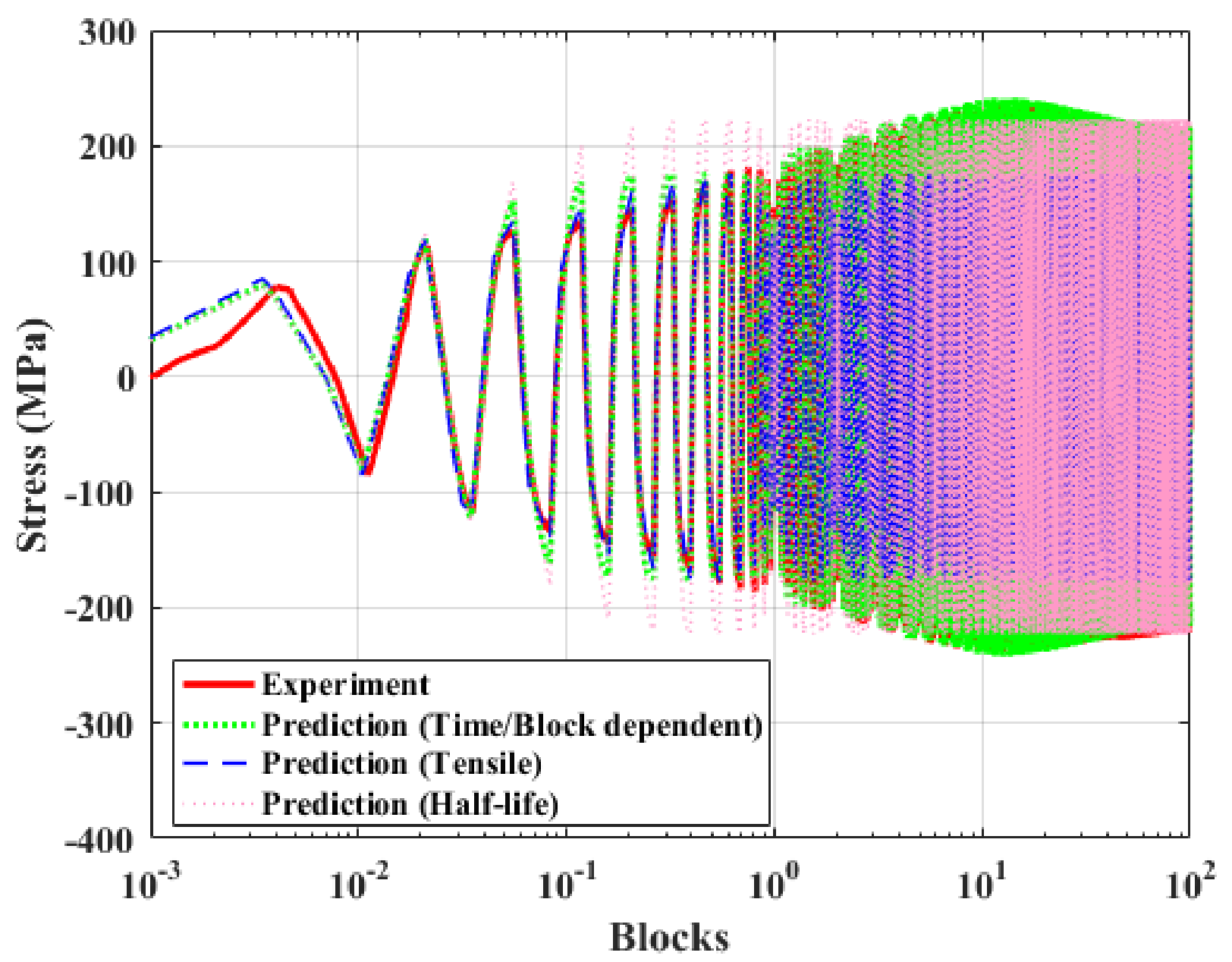

Figure 5.10 3D-FE simulated (evolutionary cycle plasticity model) vs. experimental axial stress of ET-F38 specimen for first 50 blocks. Predictions are from simulation using time/block-dependent parameters (ET-F38) and two sets of time-independent parameters (tensile test ET-T04 and half-life block of ET-F38). 


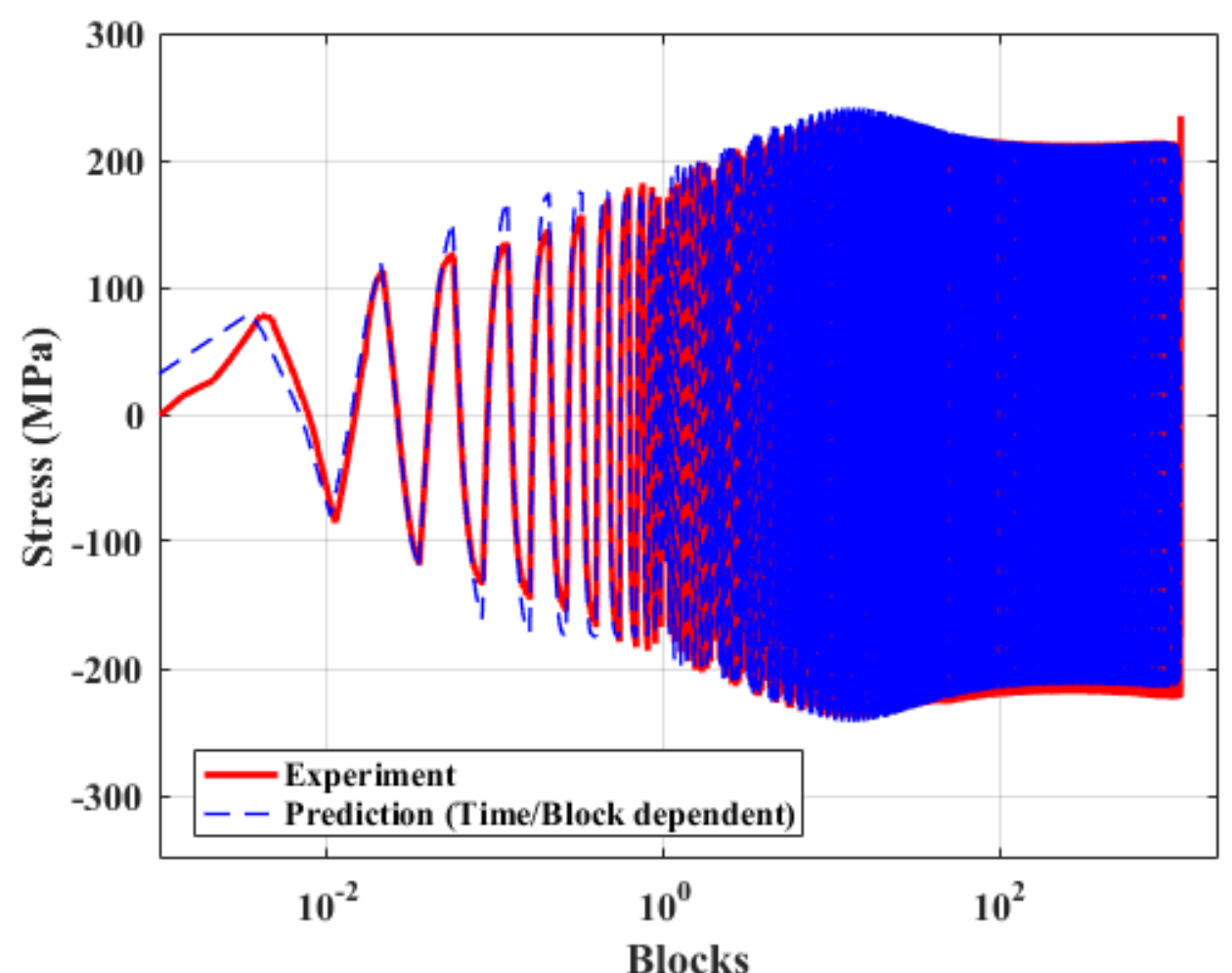

Figure 5.11 3D-FE simulated (evolutionary cycle plasticity model) vs. experimental axial stress history of ET-F38 specimen for whole fatigue life.

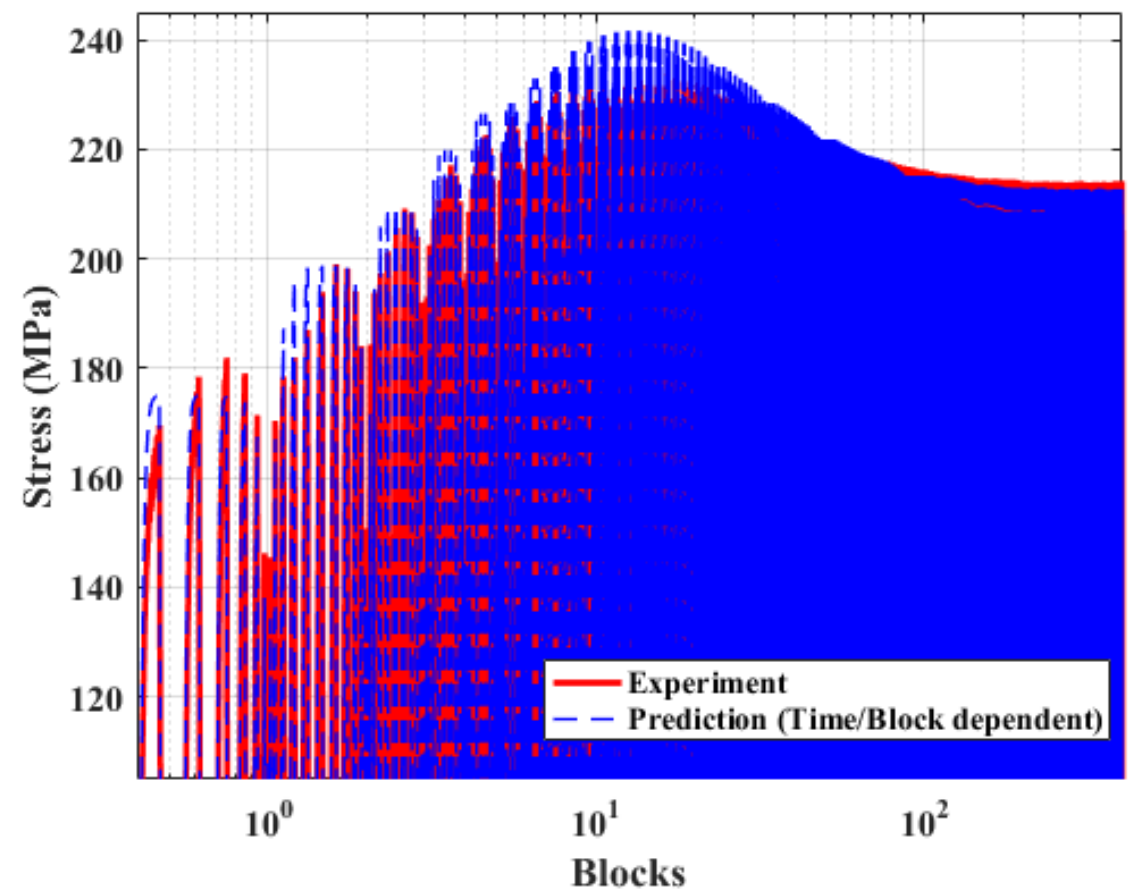

Figure 5.12 Magnified version of Figure 5.11 showing that the 3D-FE results can predict material behavior during initial stress hardening, softening, and stabilized cycles under variable-amplitude loading. 


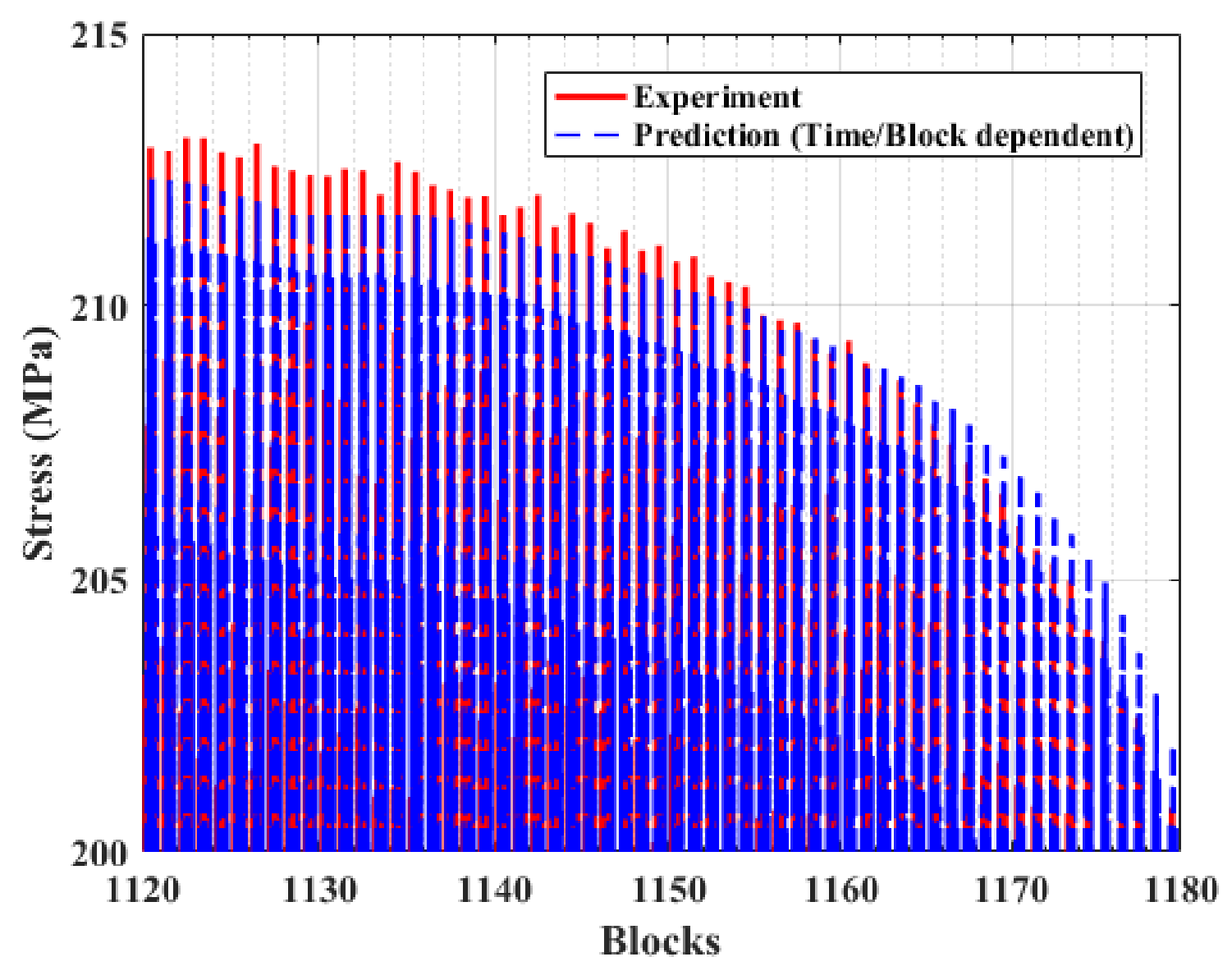

Figure 5.13 Magnified version of Figure 5.11 showing that the 3D-FE results can predict material behavior during rapid crack propagation and failure under variable-amplitude loading.

\subsection{Results from APSE-Based 3D-FE Models}

\subsubsection{Constant-Amplitude Fatigue Test}

The constant-amplitude fatigue tests ET-F41 and ET-F06 were 3D-FE modeled by using the ET-F38 material parameters as a function of APSE. The predicted axial stress profile from the 3D-FE simulation was compared with the experimentally observed stress profiles from ET-F41 and ET-F06. Comparison plots are shown in Figures 5.14 and 5.15 for ET-F41 and ET-F06, respectively. Note that the amplitude condition was the same for both tests, and thus the predicted stress profiles shown in Figures 5.14 and 5.15 are the same, but the experimental stress profiles are different. As seen from the figures, the simulated stress profile exhibits all the characteristic behavior (initial hardening followed by softening and stabilized cycles and rapid crack propagation toward the end of fatigue life) of 316 SS under constant-amplitude fatigue load. However, the APSE-based prediction is not as close to the experimental data as the timebased prediction. For example, the maximum hardening stress occurs at the $53^{\text {rd }}$ cycle during ET-F06 and ET-F41) but at the $69^{\text {th }}$ cycle in the 3D-FE results. Also, the predicted fatigue life from the simulation was 
found to be 4864 cycles, which is between the experimentally observed fatigue lives from the two experiments (6914 cycles for ET-F41 and 4202 cycles for ET-F06). See Section 5.3 for a complete comparison between the time-based and APSE-based 3D-FE predictions.

For the time-based modeling, the material parameters were estimated from the same test, and therefore, the prediction was very close to experimental data. By contrast, the material parameters used for APSEbased modeling were estimated from a different test (variable-amplitude test ET-F38), and thus, the prediction was not as accurate as for time-based modeling. However, considering the variation in experimentally observed fatigue lives between two constant-amplitude tests with the same condition, the APSE-based prediction was not bad at all. Moreover, APSE-based modeling provides the robustness of predicting material behavior under any type of amplitude loading, including random. The APSE-based 3D-FE modeling of random-amplitude test ET-F40 is discussed in Section 5.2.3.

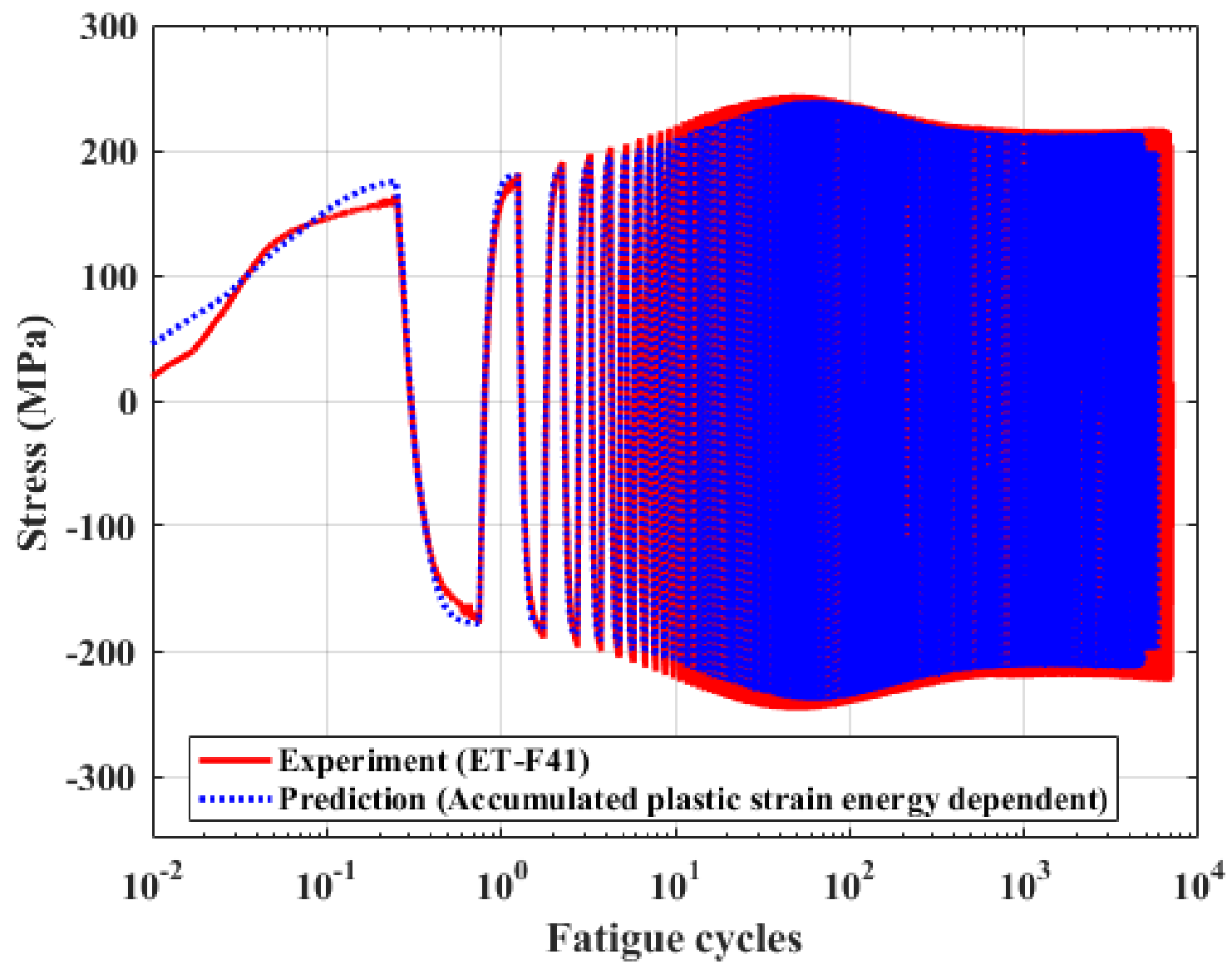

Figure 5.14 3D-FE simulated (evolutionary cycle plasticity model) vs. experimental axial stress history of ET-F41 specimen for whole fatigue life. Prediction is from simulation using APSE-dependent material parameters estimated from variable-amplitude test (ET-F38). 


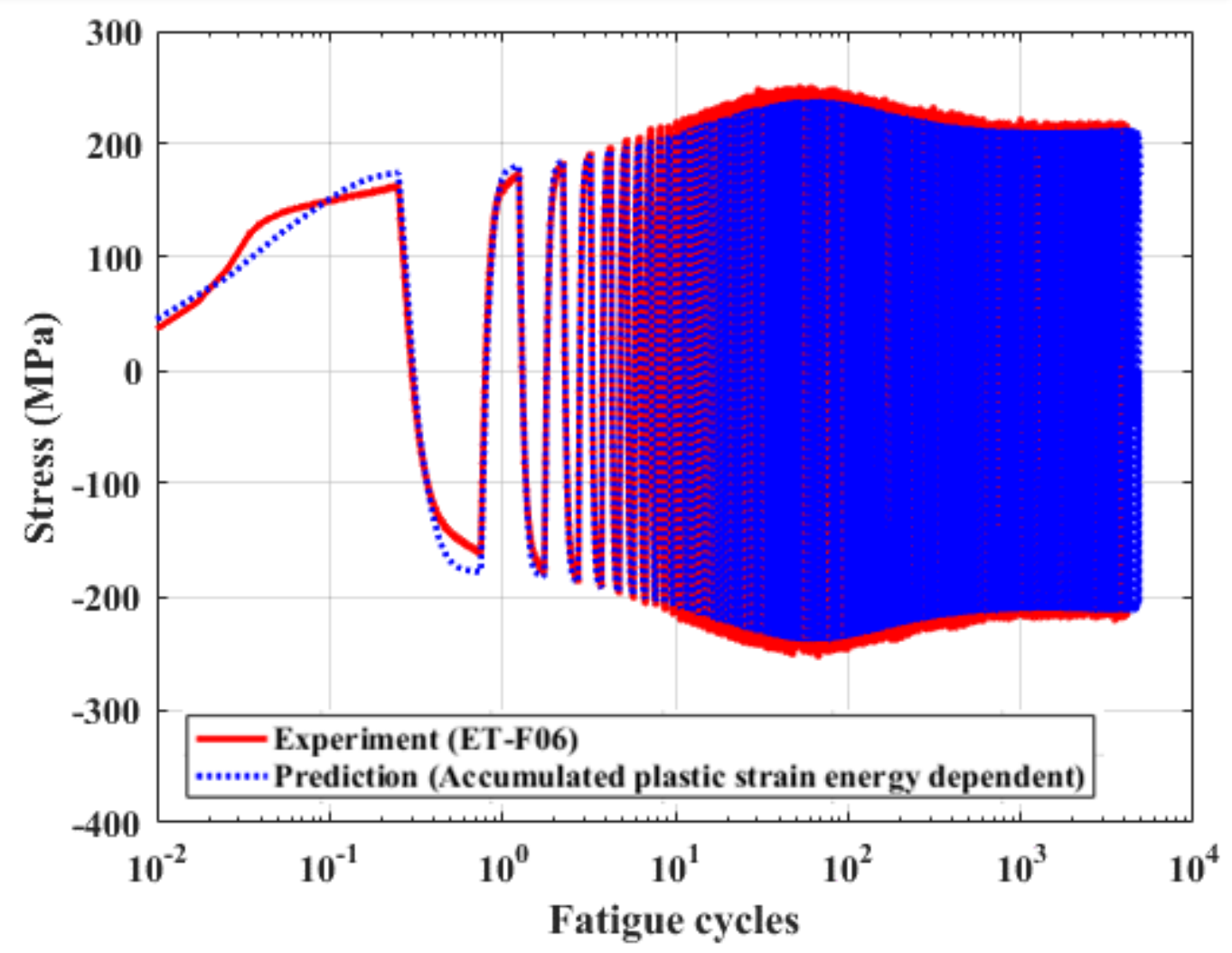

Figure 5.15 3D-FE simulated (evolutionary cycle plasticity model) vs. experimental axial stress history of ET-F06 specimen for whole fatigue life. Prediction is from simulation using APSE-dependent material parameters estimated from variable-amplitude test (ET-F38).

\subsubsection{Variable-Amplitude Fatigue Test}

Figure 5.16 compares the experimental stress history observed from the variable-amplitude test ETF38 and the 3D-FE simulated stress using the evolutionary cyclic plasticity model and the APSEdependent material parameters. As seen from the figure, the simulated stress profile exhibits all the characteristic behavior (initial hardening followed by softening and stabilized cycles and rapid crack propagation toward the end of fatigue life) of $316 \mathrm{SS}$ under variable-amplitude fatigue load. The accuracy of the prediction in terms of maximum hardening stress and fatigue life will be discussed Section 5.3. 


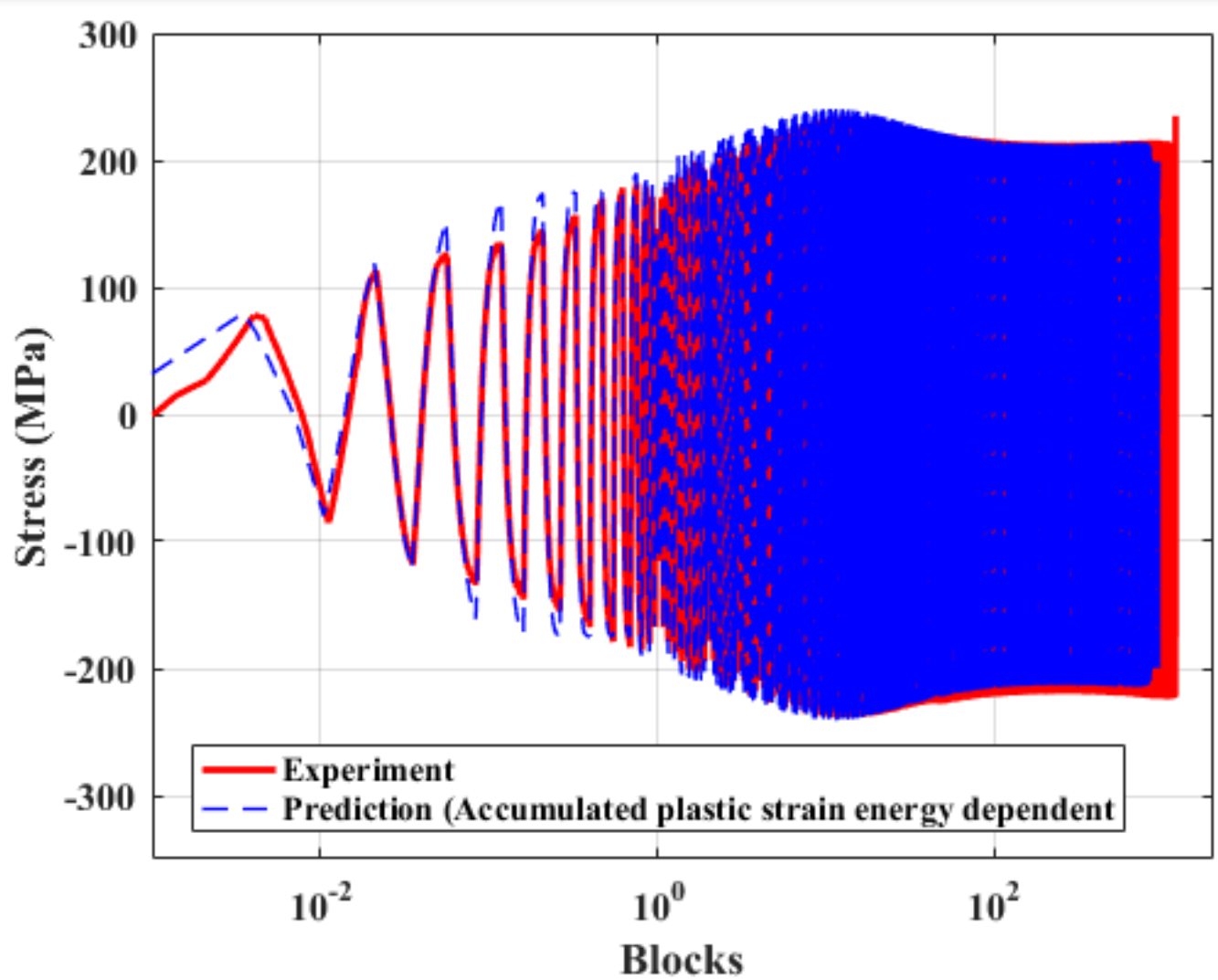

Figure 5.16 3D-FE simulated (evolutionary cycle plasticity model) vs. experimental axial stress history of ET-F38 specimen for whole fatigue life. Prediction is from simulation using APSE-dependent material parameters estimated from variable-amplitude test (ET-F38).

\subsubsection{Random-Amplitude Fatigue Test}

The random-amplitude fatigue test ET-F40 was 3D-FE modeled with the evolutionary cyclic plasticity model and the APSE-dependent material parameters estimated from variable-amplitude fatigue test ETF38. The ET-F40 test was also 3D-FE modeled with fixed material parameters estimated from tensile test ET-T04. The 3D-FE simulated axial stress profiles for the $1^{\text {st }}$ block (see Figure 3.22 for block strain input) along with the experimentally observed stress history is shown in Figure 5.17. The figure shows that the APSE-based evolutionary cyclic plasticity model can accurately predict material behavior during initial stress hardening and following stress softening, whereas the model based on fixed material properties cannot. A magnified version of Figure 5.17 is shown in Figure 5.18. The experimental and predicted stresses shown in this figure correspond to the strain input in Figure 3.24 (see Section 3.4). The corresponding stress-strain hysteresis plots are shown in Figure 5.18. Figures 5.18 and 5.19 depict the intricacy in the stress-strain variation during random loading. Despite this variation, the FE model prediction of stress response is reasonably good. For this test case, the FE simulation of the entire test was performed with APSE-dependent material properties only. The 3D-FE simulated axial stress along with experimentally observed stress for the entire fatigue life of the ET-F40 specimen is shown in Figure 5.20. As seen from this figure, APSE-based 3D-FE modeling can predict the behavior of 316 SS under random- 
amplitude loading with good accuracy. The experimentally observed and predicted life from 3D-FE simulation is discussed in Section 5.3.

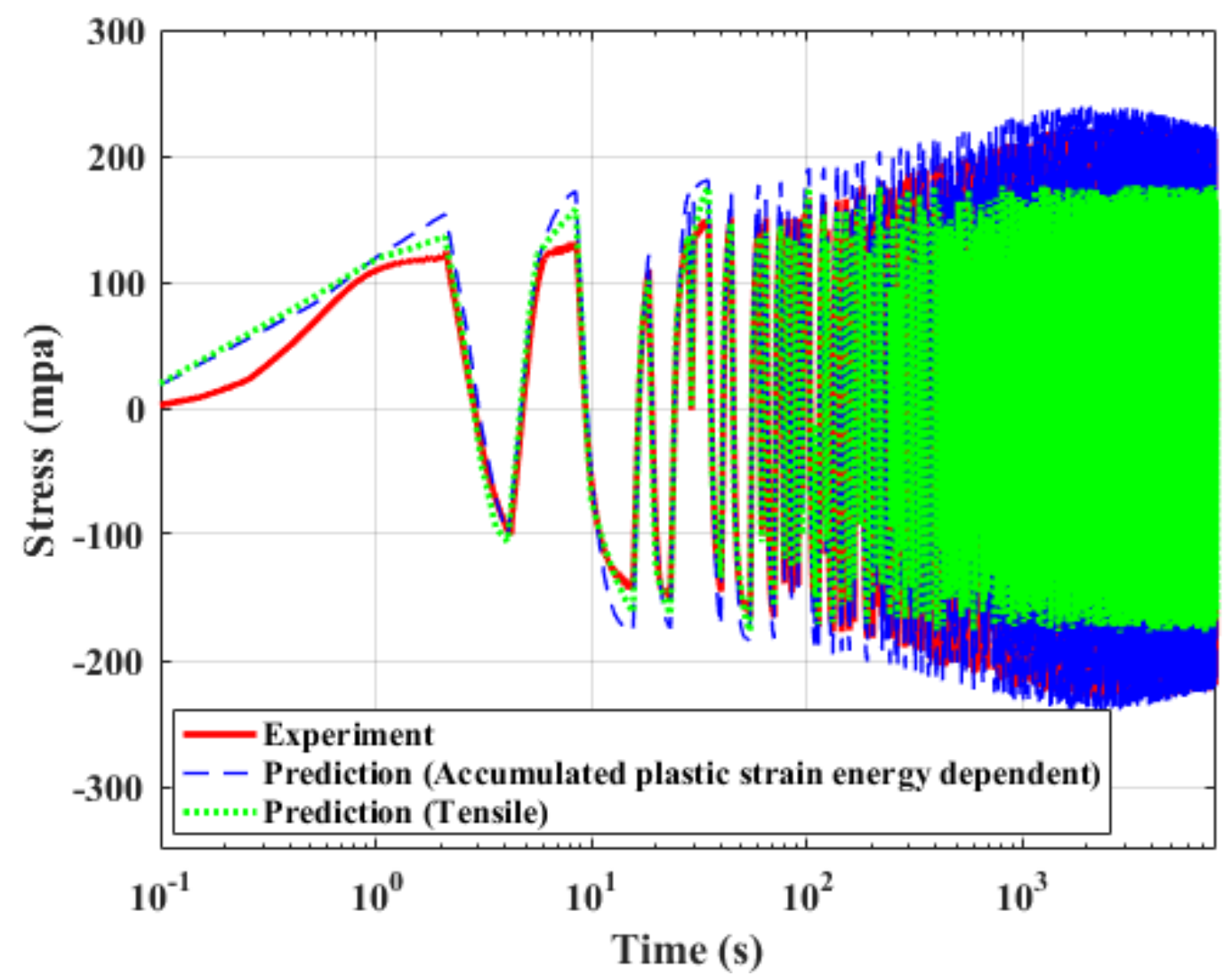

Figure 5.17 3D-FE simulated (evolutionary cycle plasticity model) vs. experimental axial stress of ET-F40 specimen during $1^{\text {st }}$ block (block period $=4251 \mathrm{~s}$ ) loading. Predictions are from simulation using APSE-dependent material parameters estimated from variable-amplitude test (ET-F38) and fixed parameters estimated from tensile test (ET-T04). 


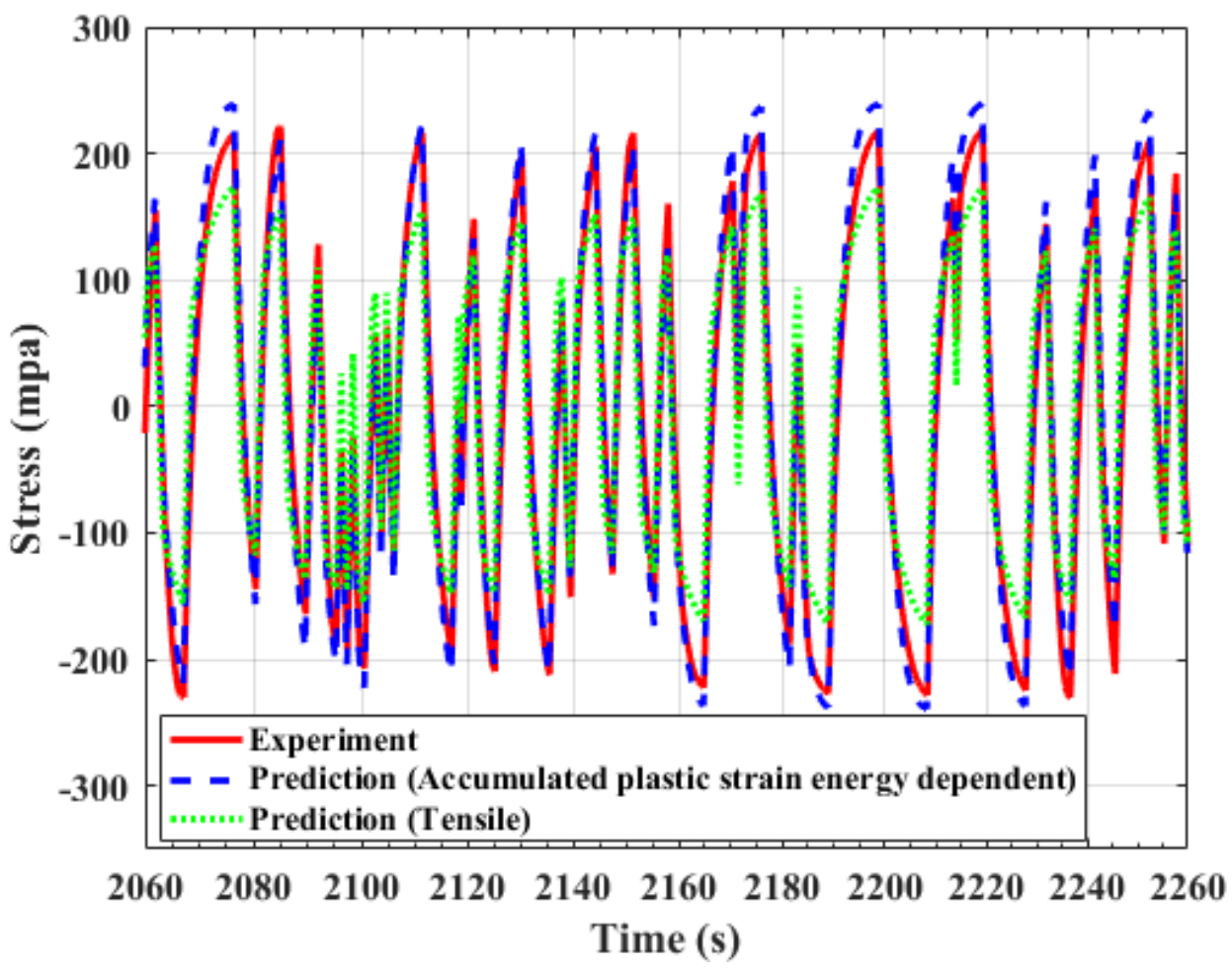

Figure 5.18 Magnified version of Figure 5.17. Experimental and predicted stress correspond to applied strain shown in Figure 3.24.

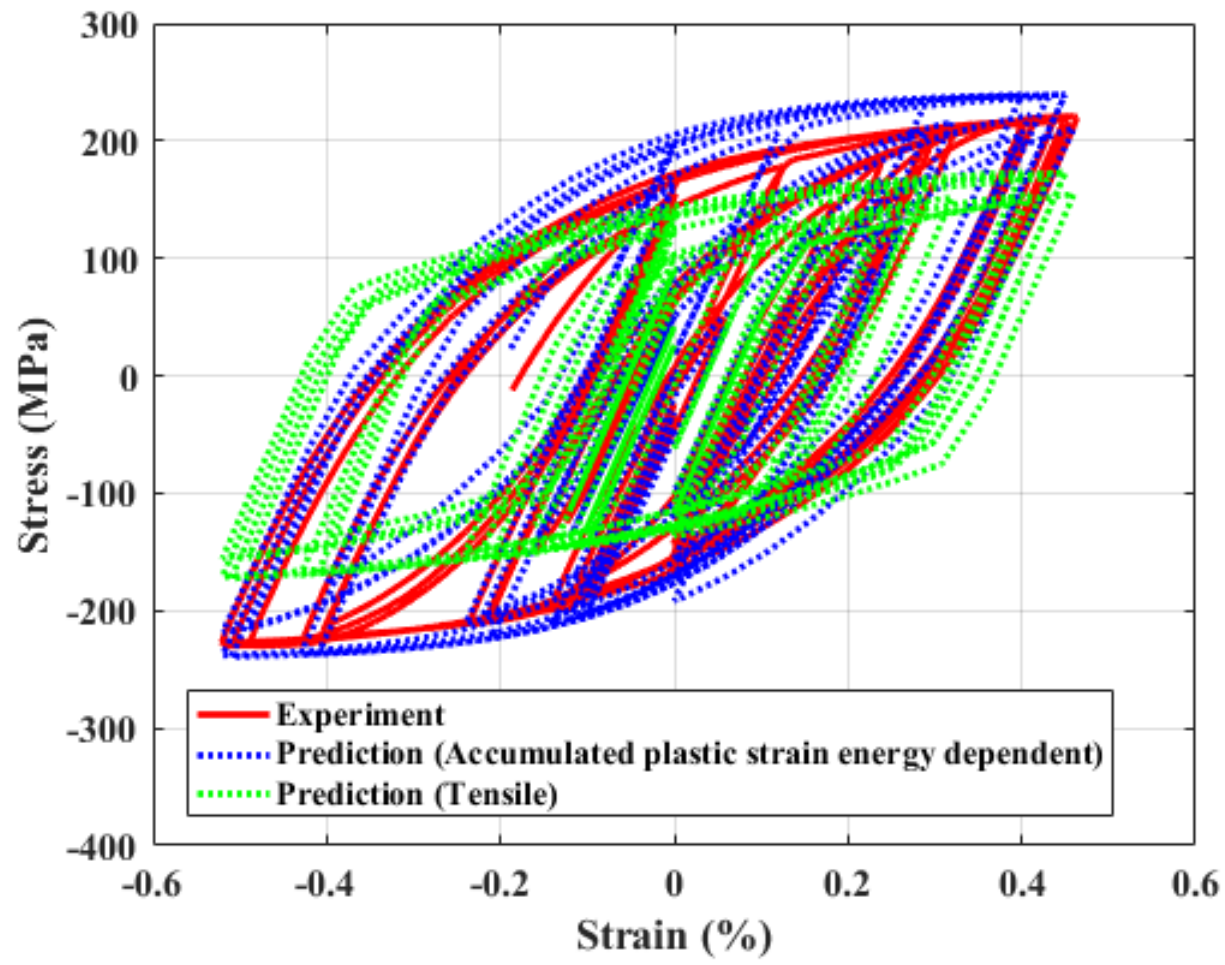

Figure 5.19 3D-FE simulated (evolutionary cycle plasticity model) vs. experimental hysteresis plot of stress shown in Figure 5.18 and strain shown in Figure 3.24. 


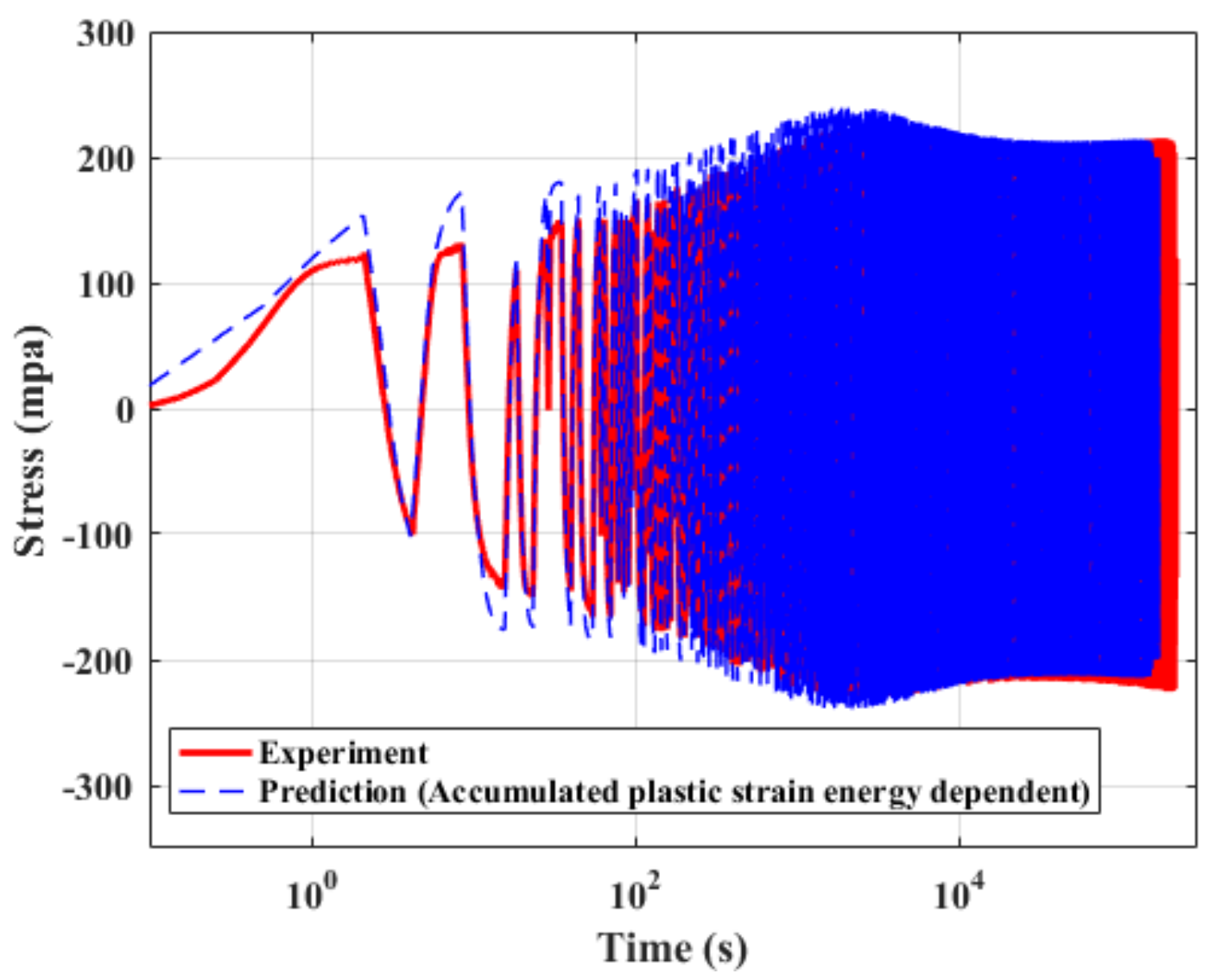

Figure 5.20 3D-FE simulated (evolutionary cycle plasticity model) vs. experimental axial stress history of ET-F40 specimen for whole fatigue life. Prediction is from simulation using APSE-dependent material parameters estimated from variable-amplitude test (ET-F38).

\subsection{Summary of FE Modeling Results and Comparison with Analytical Modeling Results}

Results from the 3D-FE modeling are summarized here to provide an overall picture of the predicted results and to compare them with the experimental results and analytical modeling results. Table 5.1 compares the experimentally observed maximum hardening stresses during all the fatigue tests with those predicted through time-based and APSE-based 3D-FE modeling. As seen from the table, the maximum hardening stress predicted through 3D-FE simulation is more than $90 \%$ accurate for all the fatigue test cases. However, the time-based prediction is more accurate, in general, as the material parameters used for modeling were estimated from the same test. Similar results were also found from analytical modeling of the fatigue tests (see Table 4.1 in Section 4.3) 
Table 5. 1 Experimentally observed and predicted (3D-FE modeling) maximum hardening stress.

\begin{tabular}{|c|c|c|c|c|c|c|}
\hline \multirow{3}{*}{$\begin{array}{l}\text { Test } \\
\text { ID }\end{array}$} & \multirow{3}{*}{$\underset{\text { type }}{\text { Amplitude }}$} & \multirow{3}{*}{$\begin{array}{c}\text { Experimental } \\
\text { observation }\end{array}$} & \multicolumn{4}{|c|}{ FE Modeling } \\
\hline & & & \multicolumn{2}{|c|}{ Time-based prediction } & \multicolumn{2}{|c|}{ APSE-based prediction } \\
\hline & & & $\begin{array}{l}\text { Stress } \\
\text { (MPa) }\end{array}$ & $\begin{array}{c}\text { Accuracy } \\
(\%)\end{array}$ & $\begin{array}{l}\text { Stress } \\
\text { (MPa) }\end{array}$ & $\begin{array}{c}\text { Accuracy } \\
(\%)\end{array}$ \\
\hline ET-F06 & Constant & 250.0 & 252.6 & 99.0 & \multirow{2}{*}{239.9} & \multirow{2}{*}{96.8} \\
\hline ET-F41 & Constant & 245.5 & 251.2 & 97.7 & & \\
\hline ET-F38 & Variable & 234.9 & 241.6 & 97.1 & 240.3 & 97.7 \\
\hline ET-F40 & Random & 223.5 & N/A ${ }^{*}$ & $N / A^{*}$ & 238.2 & 93.4 \\
\hline
\end{tabular}

\# An average experimental maximum hardening stress (247.8 MPa) based on ET-F06 and ET-F41 tests is used to calculate the accuracy in predicted maximum hardening stress.

* Time-based modeling is not possible for random amplitude loading.

In this work, stress drop to $200 \mathrm{MPa}$ was used as the failure criterion for determining the fatigue lives of the specimens. See Section 4.3 for a discussion of the failure criterion. The experimental and predicted lives for all fatigue tests and the 3D-FE modeling cases are given in Table 5.2. As seen from the table, the 3D-FE simulation based on evolutionary cyclic plasticity model predicts the fatigue life of the specimen with an accuracy more than $70 \%$. By contrast, the 3D-FE model based on time-independent or fixed material parameters predicts infinite fatigue life for all cases. The accuracy in predicted life from timebased 3D-FE simulation (i.e., using time-dependent material properties) is almost $100 \%$ for both constantand variable-amplitude fatigue tests as the material parameters were estimated from the same test. Similar results were also found from time-based analytical modeling results (see Table 4.3 in Section 4.3). However, there is a big difference in APSE-based life predictions between analytical modeling and 3DFE modeling results. A comparative study between APSE-based analytical modeling and 3D-FE modeling results is discussed in the following part of this section. The reason behind the big difference in the simulated results is also discussed. 
Table 5. 2 Comparison between experimental and predicted (3D-FE modeling) fatigue lives.

\begin{tabular}{|c|c|c|c|c|c|c|c|c|}
\hline \multirow{3}{*}{$\begin{array}{l}\text { Test } \\
\text { ID }\end{array}$} & \multirow{3}{*}{$\underset{\text { type }}{\text { Amplitude }}$} & \multirow{3}{*}{$\begin{array}{l}\text { Fatigue } \\
\text { life unit }\end{array}$} & \multirow{3}{*}{$\begin{array}{c}\text { Experiment } \\
\begin{array}{c}\text { Observed } \\
\text { life }\end{array} \\
\end{array}$} & \multicolumn{5}{|c|}{ FE Modeling } \\
\hline & & & & \multirow{2}{*}{$\begin{array}{c}\begin{array}{c}\text { Fixed } \\
\text { properties } \\
\text { (tensile/half- } \\
\text { life) }\end{array} \\
\text { Predicted life }\end{array}$} & \multicolumn{2}{|c|}{ Time-based } & \multicolumn{2}{|c|}{ APSE-based } \\
\hline & & & & & $\begin{array}{l}\text { Predicted } \\
\text { Life }\end{array}$ & $\begin{array}{c}\text { Accuracy } \\
(\%)\end{array}$ & $\begin{array}{l}\text { Predicted } \\
\text { Life }\end{array}$ & $\begin{array}{c}\text { Accuracy } \\
(\%)\end{array}$ \\
\hline $\begin{array}{l}\text { ET- } \\
\text { F06 }\end{array}$ & Constant & Cycles & 4202 & $\infty$ & 4200 & 100.0 & \multirow{2}{*}{4864} & \multirow{2}{*}{$87.5^{\#}$} \\
\hline $\begin{array}{l}\text { ET- } \\
\text { F41 }\end{array}$ & Constant & Cycles & 6914 & $\infty$ & 6925 & 99.8 & & \\
\hline $\begin{array}{l}\text { ET- } \\
\text { F38 }\end{array}$ & Variable & Blocks & 1179 & $\infty$ & 1180 & 99.9 & 867 & 73.5 \\
\hline $\begin{array}{l}\text { ET- } \\
\text { F40 }\end{array}$ & Random & Time (s) & 21590 & $\infty$ & N/A & N/A & 15860 & 73.5 \\
\hline
\end{tabular}

* Time-based modeling is not possible for random amplitude loading. \# APSE-based FE simulation was not performed for ET-F38 test.

\# An average experimental life (5558 cycles) based on observation from ET-F06 and ET-F41 tests is used to calculate the accuracy in predicted life.

Table 5.3 compares the fatigue lives predicted through APSE-based analytical and 3D-FE modeling for all the different-amplitude fatigue cases. Fatigue lives determined from experimental data are also provided in the table. With analytical modeling, as shown in the Table 5.3, the APSE-based life prediction was more than $90 \%$ accurate. The predicted life was somewhat less accurate (between $70 \%$ and $90 \%$ ) in the case of APSE-based 3D-FE modeling. Note that the analytical models are mathematical models that have a closed-form solution, while the FE method uses a numerical technique to obtain an approximate solution through discretization. Thus, analytical modeling always predicts better than FE modeling. However, analytical modeling can only be done for laboratory specimens, and FE modeling is required to perform component- or system-level modeling.

To determine the reason behind the difference in APSE-based 3D-FE modeling results from analytical modeling results, values of the APSE and axial (z-direction) stress were investigated at two different nodes and compared with the values at the centroid. Figure 5.21 shows the nodes of interest in the ABAQUS model. Figures 5.22 and 5.23 shows the APSE values at those two nodes and at the centroid during the APSE-based 3D-FE simulation of fatigue tests at constant (ET-F41 or ET-F06) and random (ET-F40) amplitude. As seen from the figures, the values of APSE in the two nodes differ significantly. Since the APSE-based modeling uses the APSE in the previous steps to define the material properties for the current 
step, properties are different at different nodes. Although ABAQUS uses an average value of the nodal properties at the integration point for solving the model, the nodes with higher APSE values exhibit the fast stress drop characteristic of material fatigue behavior (i.e., rapid crack propagation and failure toward end of fatigue life of specimen) earlier than the nodes with lower APSE values. This phenomenon can be observed in Figures 5.24 and 5.25 in the case of the APSE-based simulation of fatigue tests at constant (ET-F41 or ET-F06) and random (ET-F40) amplitude, respectively. Using a higher number of nodes or more elements should reduce this difference and thus improve the accuracy of the fatigue life prediction from the APSE-based 3D-FE simulation. In addition, the results can be improved by considering the actual geometry of the specimen. This is one of our future tasks after we improve the computational hardware (e.g., with many more CPUs and/or GPUs) and software (e.g., more ABAQUS license tokens for using ABAQUS in a large number of CPUs and GPUs).

Table 5. 3 Comparison of APSE-based predicted fatigue lives between analytical modeling and 3DFE modeling results.

\begin{tabular}{|c|c|c|c|c|c|c|}
\hline \multirow{2}{*}{$\begin{array}{c}\text { Fatigue test } \\
\text { type } \\
\text { (amplitude) }\end{array}$} & \multirow{2}{*}{$\begin{array}{c}\text { Fatigue } \\
\text { life unit }\end{array}$} & Experimental & \multicolumn{2}{|c|}{ Analytical modeling } & \multicolumn{2}{|c|}{ FE modeling } \\
\cline { 3 - 7 } & Observed life & $\begin{array}{c}\text { Predicted } \\
\text { life }\end{array}$ & $\begin{array}{c}\text { Accuracy } \\
(\%)\end{array}$ & $\begin{array}{c}\text { Predicted } \\
\text { Life }\end{array}$ & $\begin{array}{c}\text { Accuracy } \\
(\%)\end{array}$ \\
\hline Constant & Cycles & $5558^{*}$ & 5860 & 94.6 & 4864 & 87.5 \\
\hline Variable & Blocks & 1179 & 1090 & 92.5 & 867 & 73.5 \\
\hline Random & Time (s) & 21590 & 20440 & 94.6 & 15860 & 73.5 \\
\hline
\end{tabular}

* An average experimental life (5558 cycles) based on observation from ET-F06 and ET-F41 tests is used. 


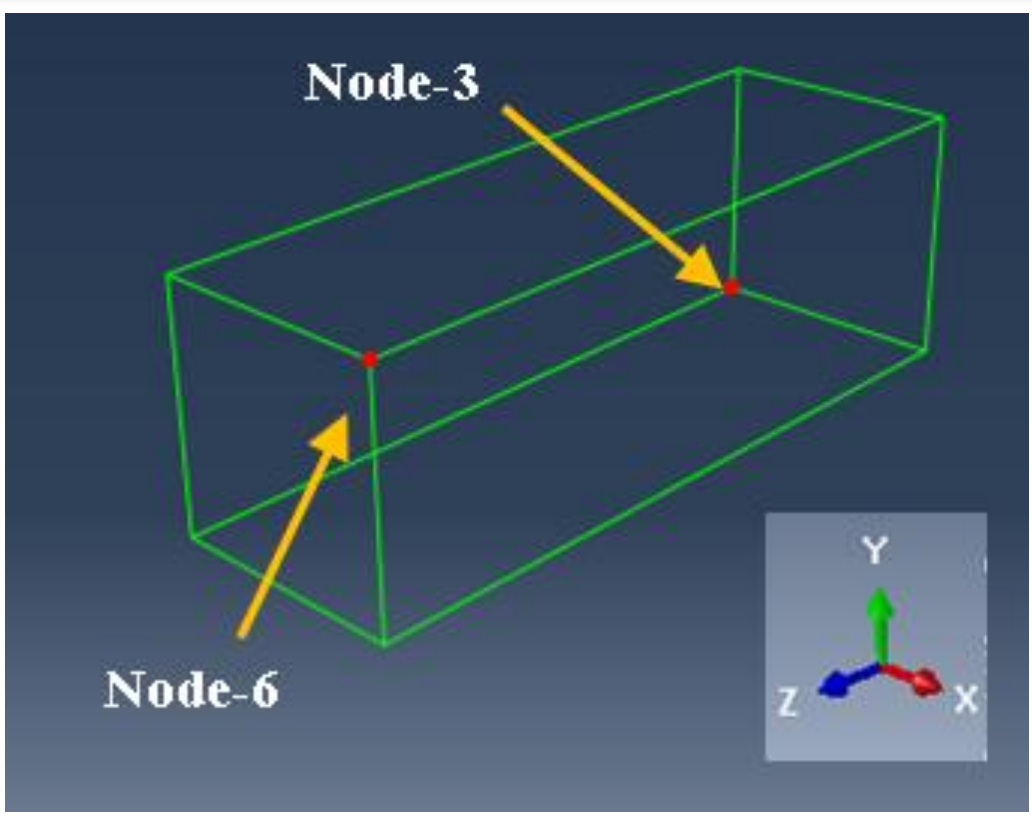

Figure 5.21 ABAQUS model used for 3D-FE simulation. Nodes indicated are points of interest to investigate the values of APSE and stress in z-direction.

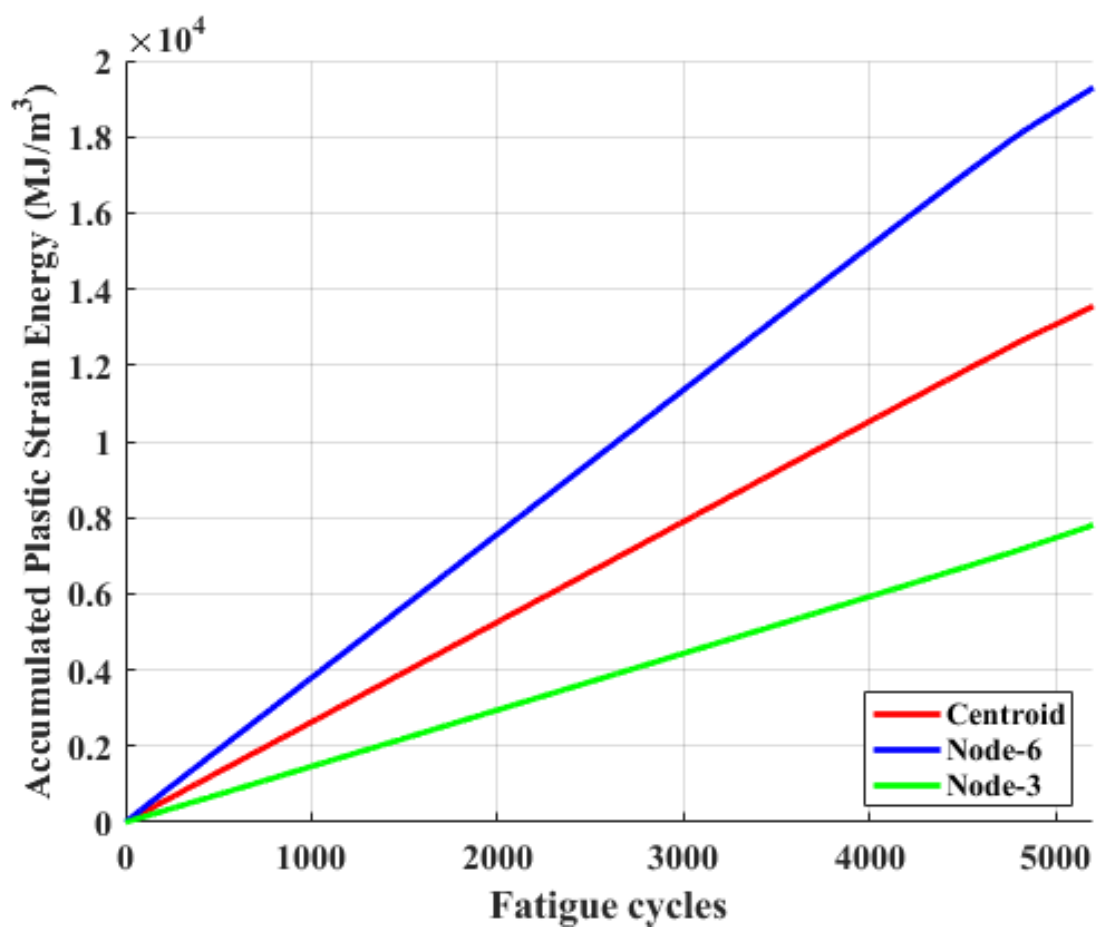

Figure 5.22 APSE as function of fatigue cycles during APSE-based 3D-FE simulation of constantamplitude fatigue test (ET-F41 and ET-F06). APSE values at node-3 and node-6 (see Figure 5.21 for node location in the ABAQUS model) are compared with that at centroid. 


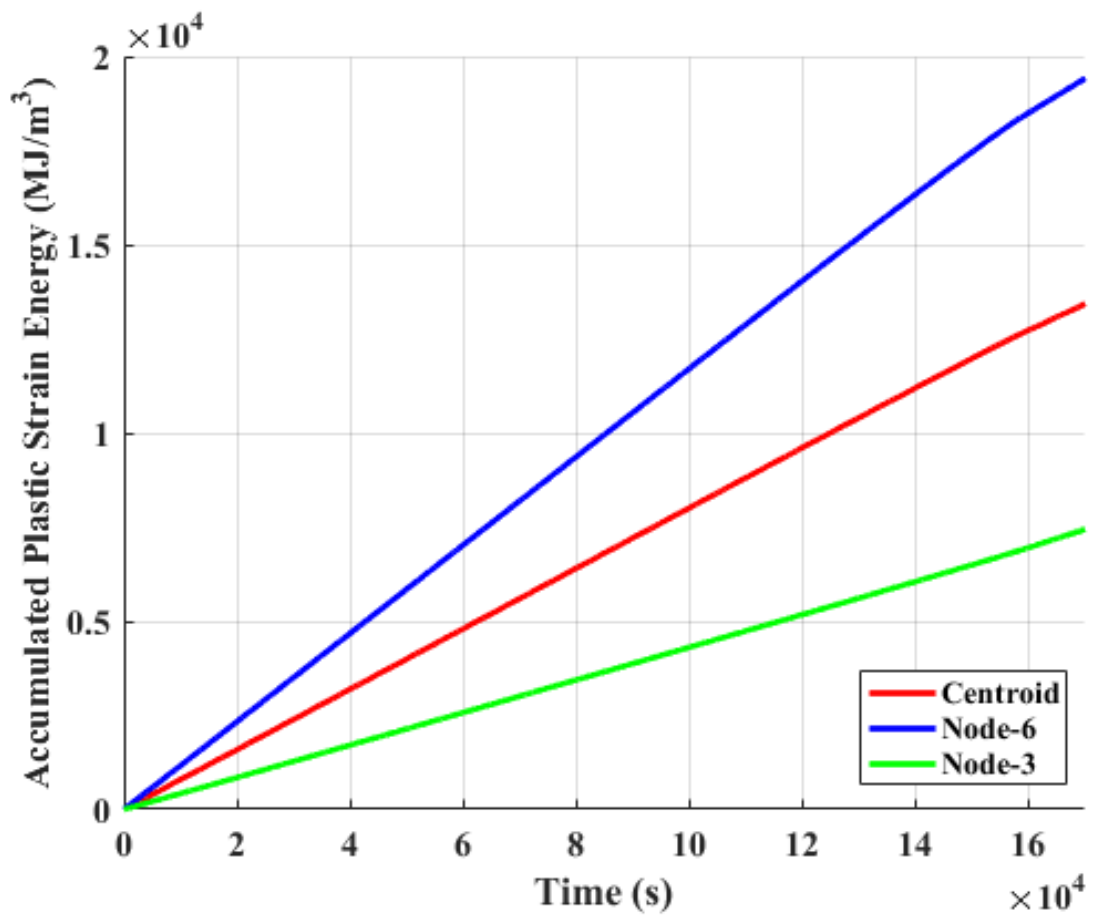

Figure 5.23 APSE as function of time during APSE-based 3D-FE simulation of random-amplitude fatigue test (ET-F40). APSE values at node-3 and node-6 (see Figure 5.21 for node location in the ABAQUS model) are compared with that at centroid.

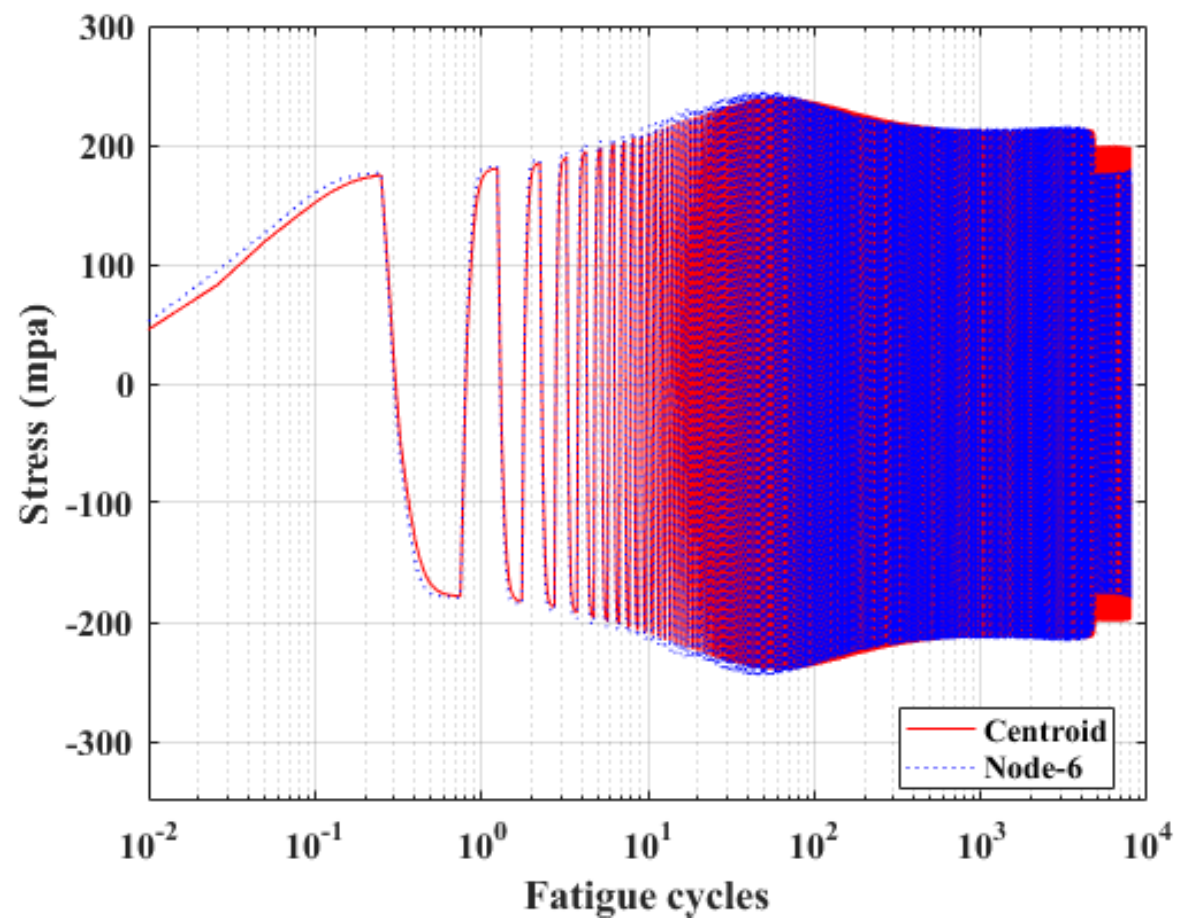

Figure 5.24 Axial stress as function of fatigue cycles during APSE-based 3D-FE simulation of constant-amplitude fatigue test (ET-F41 and ET-F06). Stress at node-6 (see Figure 5.21 for node location in the ABAQUS model) is compared with that at centroid. 

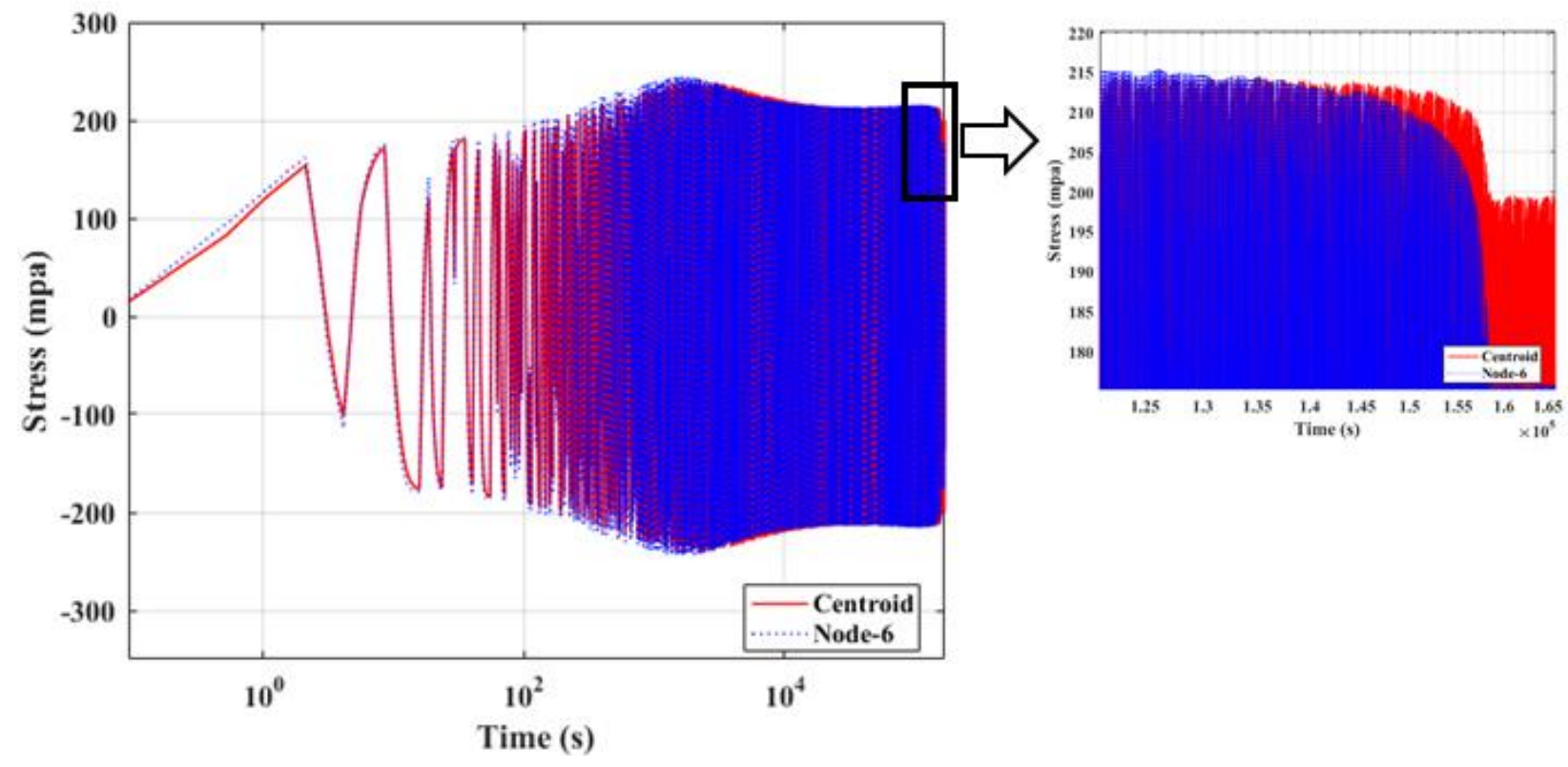

Figure 5.25 Axial stress as function of fatigue cycles during APSE-based 3D-FE simulation of random-amplitude fatigue test (ET-F40). Stress at node-6 (see Figure 5.21 for node location in the ABAQUS model) is compared with that at centroid. 


\section{Computational Fluid Dynamics Simulation of Surge Line Pipe for Thermal Stratification Evaluation}

Computational fluid dynamics (CFD) models of a PWR surge line (SL) are being developed for estimating the temperature boundary condition and stratification along the length of the SL pipe. This temperature data will then be used for stress analysis of SL pipe. Below, the CFD model information and some related preliminary results are presented.

\subsection{CFD: Theoretical Background}

In the reported work, commercially available ABAQUS software was used for the CFD simulation. The details of the CFD theoretical background and its implementation to ABAQUS code can be found in the ABAQUS user manual [38] or elsewhere. However, in this subsection, we briefly describe the theoretical information for completeness and easy reference of the terminology and material constants used in the model. In this CFD model, an implicit large-eddy simulation (ILES) is used. Note that the ILES is a technique for modeling high Reynolds number flows, is extremely flexible for implementation, and can be applied to a broad range of flow modeling [38]. The related conservation of mass, momentum, and energy equations are briefly described below.

The conservation of mass equation can be given as:

$$
\frac{\partial \rho}{\partial t}+\nabla(\rho v)=0
$$

where $\nabla, \rho$, and $v$ are, respectively, the del operator, density, and velocity of the fluid. Then, the conservation of momentum equation can be given as:

$$
\frac{\partial}{\partial t}(\rho v)+\nabla(\rho v \otimes v)=-\nabla p+\nabla\left(\mu\left(\nabla v+(\nabla v)^{T}\right)+\rho g\right.
$$

where $\Theta, p, \mu$, and $g$ are, respectively, the outer product, pressure, dynamic viscosity, and acceleration due to gravity. For CFD simulation involving non-isothermal conditions (such as in the present case of SL temperature stratification), the energy equation has to be activated. The corresponding energy conservation equation is given as:

$$
\frac{\partial}{\partial t}\left(\rho C_{p} \theta\right)+\nabla\left(\rho C_{p} \theta v\right)=Q+k_{f} \nabla \theta
$$

where $C_{p}, \theta, Q$, and $k_{f}$ are, respectively, the specific heat or heat capacity at constant pressure, temperature, external heat supplied, and the coefficient of thermal conductivity. In Eqs. 6.1-6.3 the density $(\rho)$, dynamic viscosity $(\mu)$, specific heat capacity at constant pressure $\left(C_{p}\right)$, and thermal conductivity $\left(k_{f}\right)$ are material constants. By contrast, other variables are either supplied as boundary conditions or estimated with the CFD model. Also, note that an incompressible flow condition is assumed in the reported CFD simulation. 


\subsection{Surge line Pipe CFD Model}

\subsubsection{Boundary Conditions}

The SL pipe of a four-loop type PWR was CFD modeled to estimate the wall temperature and thermal stratification (if any) along the length of the SL line. In a PWR the SL pipe normally connects the hot leg and pressurizer. The major aim of the SL along with the pressurizer is to maintain the primary loop pressure of the PWR. Figure 6.1 shows the ABAQUS model, representing the surge line, hot leg (HL), and part of the pressurizer. For the CFD model we had originally planned to simulate the complete assemble shown in Figure 6.1. However, to reduce the computational time, only the surge line section of the pipe was modeled. Figure 6.2 shows the mesh of the surge line and locations of the applied temperature and pressure-velocity boundary condition. The simulation was conducted to estimate the wall temperature of the SL under a typical heat-up, cool-down, and steady-state power operation. Figure 6.3 shows the temperature boundary conditions applied for the SL-pressurizer and SL-HL nozzles. In designing the simplified temperature boundary condition shown in Figure 6.3, the heat-up and cool-down temperature rates were maintained within the limiting rate of $100^{\circ} \mathrm{F} / \mathrm{hr}$, which is typically followed in a Westinghouse type reactor [39]. The temperature of the reactor cooling system at the end of Mode 3 (hot standby 3) was chosen as $557{ }^{\circ} \mathrm{F}\left(291.667{ }^{\circ} \mathrm{C}\right)$. At the end of Mode 3, we assumed that the pressurizer would reach its maximum pressure of $2235 \mathrm{psi}(15.41 \mathrm{MPa})$ with a saturated water temperature of $651.784^{\circ} \mathrm{F}(344.324$ $\left.{ }^{\circ} \mathrm{C}\right)$. From the hot standby condition, the HL temperature was increased to $691.52^{\circ} \mathrm{F}\left(326.4{ }^{\circ} \mathrm{C}\right)$, which is the typical temperature of a 4-loop type PWR at $100 \%$ thermal power [40]. After the hot standby condition was achieved, the pressurizer was maintained at a steady-state temperature of $651.784{ }^{\circ} \mathrm{F}\left(344.324{ }^{\circ} \mathrm{C}\right)$. A temperature of $651.784{ }^{\circ} \mathrm{F}\left(344.324{ }^{\circ} \mathrm{C}\right)$ for the pressurizer and $691.52{ }^{\circ} \mathrm{F}\left(326.4{ }^{\circ} \mathrm{C}\right)$ for the $\mathrm{HL}$ was maintained during the steady-state power operation, and then it was reduced in a similar manner (or rate) to that during heat-up operations (refer to Figure 6.3). Note that in a real reactor, the heat-up and cooldown operations are conducted in multiple steps, and in each step some waiting time is included to check the performance of different equipment. However, in the present work to reduce the simulation time, simplified boundary transients were selected, as shown in Figure 6.3. Also note that the duration of the power operation for a real plant is in months (approximately 1 year for one fuel cycle). However, in the presented case, a perfect steady-state condition and a shorter duration of few hours were considered (refer to Figure 6.3).

The total mass flow rate for the assumed 4-loop PWR was considered (based on the information given in [3]) equal to $17,417.95 \mathrm{~kg} / \mathrm{sec}$. With this information the maximum mass flow rate in one loop and the corresponding hot leg was considered equal to $4354.49 \mathrm{~kg} / \mathrm{s}$. A time-dependent velocity profile (with a minimum mass flow rate of $2 \mathrm{gal} / \mathrm{min}$ at initial condition) was estimated on the basis of this flow rate and the temperature-dependent density considered. The estimated velocity profile is shown in Figure 6.4. This velocity profile was applied to the SL-HL nozzle as the velocity boundary condition perpendicular to the SL axis at the SL-HL nozzle. In addition, the lower mass flow rate of $2 \mathrm{gal} / \mathrm{min}(0.126 \mathrm{~L} / \mathrm{s})$ is assumed to exist at the SL-pressurizer nozzle. Note that at the steady-state condition in a spray line, the flow rate of the bypass flow that is used to cool the pressurizer water when it is over heated is approximately 1.5-2 $\mathrm{gal} / \mathrm{min}$ [41]. For the present work we assumed the flow rate of the spray line is the same as that of the surge line. With the assumption of $2 \mathrm{gal} / \mathrm{min}$, an equivalent vertical velocity of $2.033 \mathrm{~mm} / \mathrm{s}$ was applied at the SL-pressurizer nozzle. The velocity was assumed constant throughout the simulation. The reference pressure of the surge line was assumed to be equal to the steady-state pressure of 15.41 MPa. 


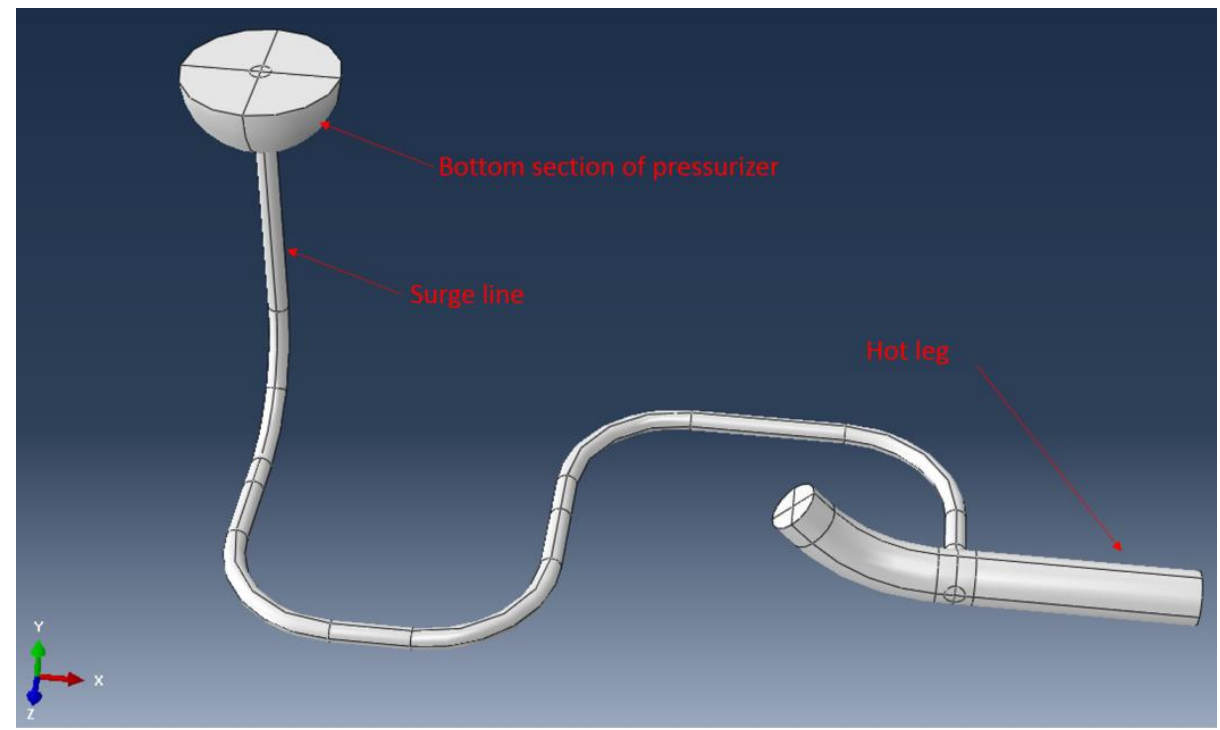

Figure 6. 1 ABAQUS model of surge line that connects the hot leg and pressurizer.

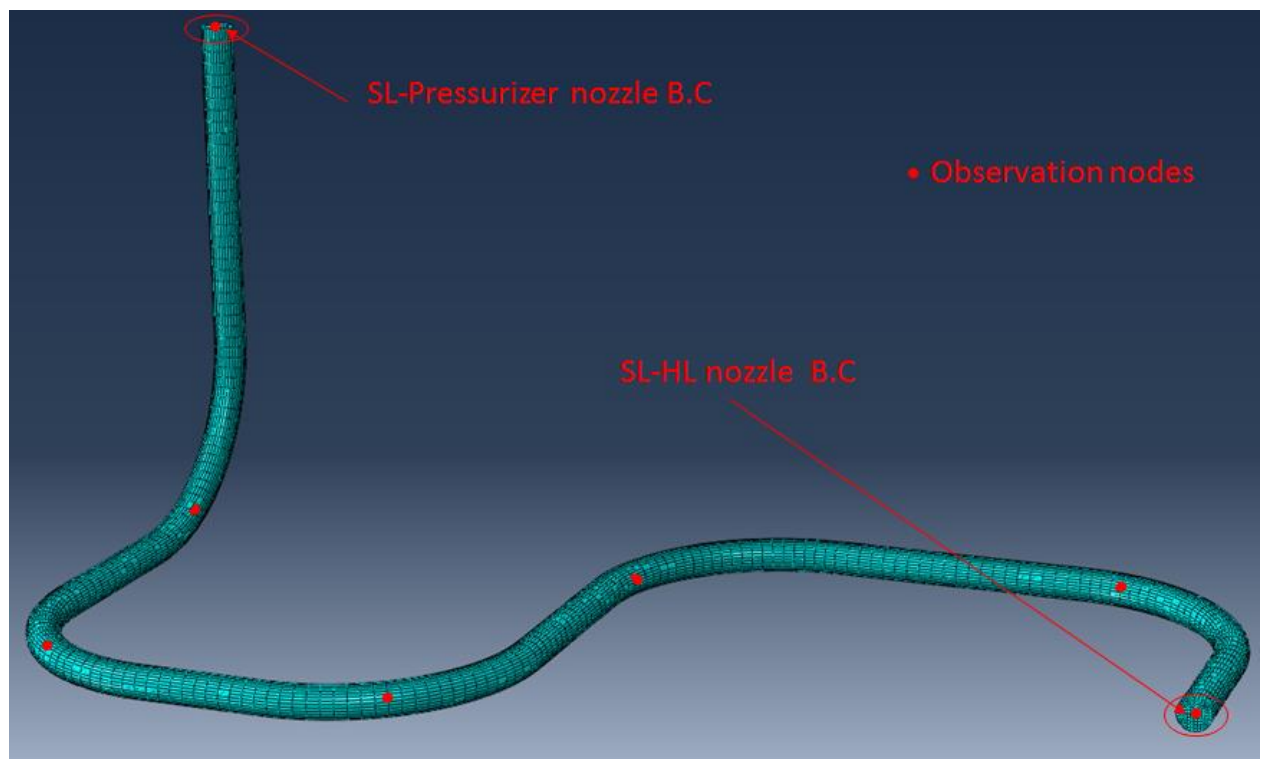

Figure 6. 2 FE mesh of the SL pipe and the locations of the applied boundary conditions. The location of few representative nodes are highlighted to show some example results discussed later. 


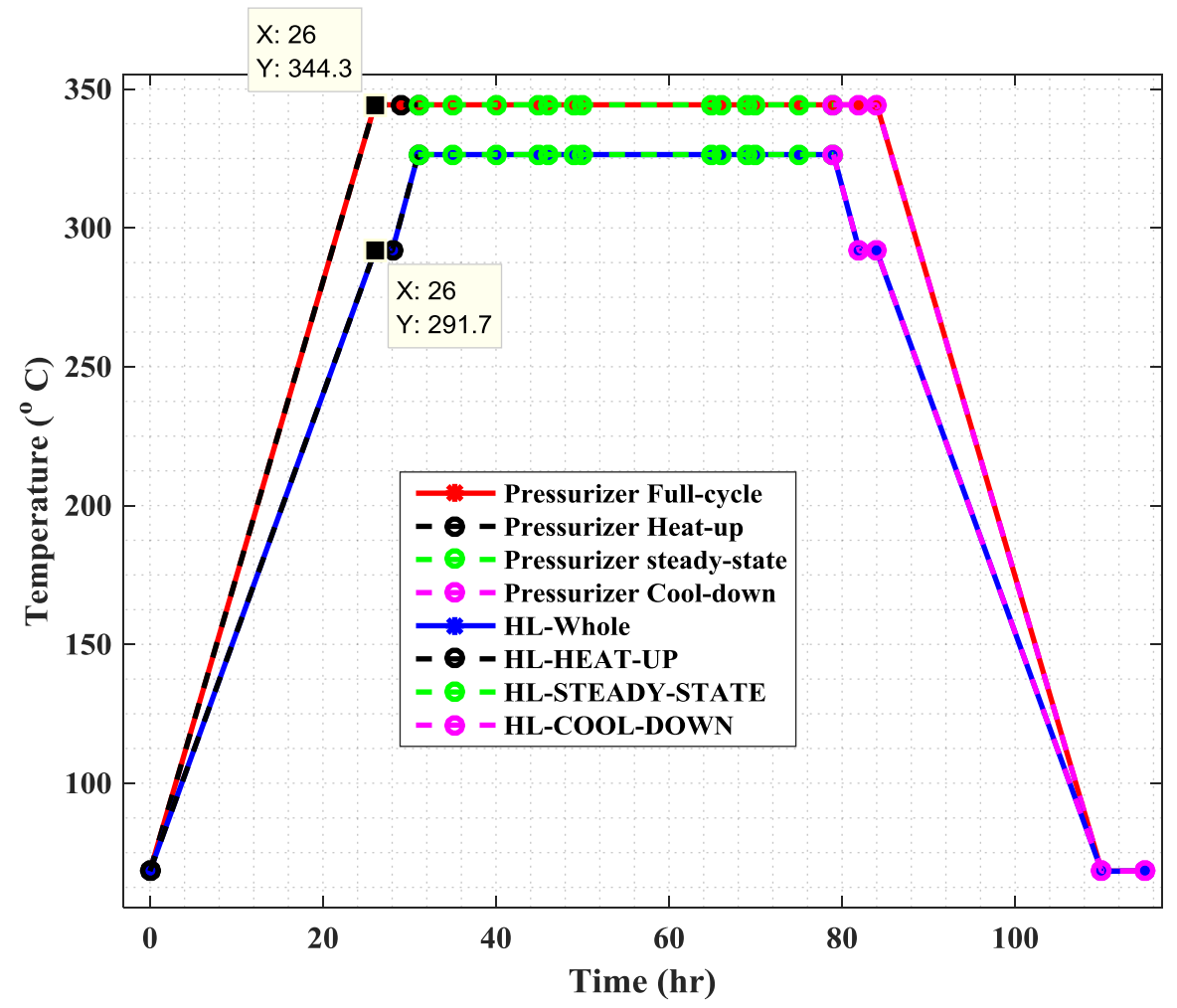

Figure 6. 3 Applied temperature boundary conditions mimicking the temperature at SL-pressurizer and SL-HL nozzle.

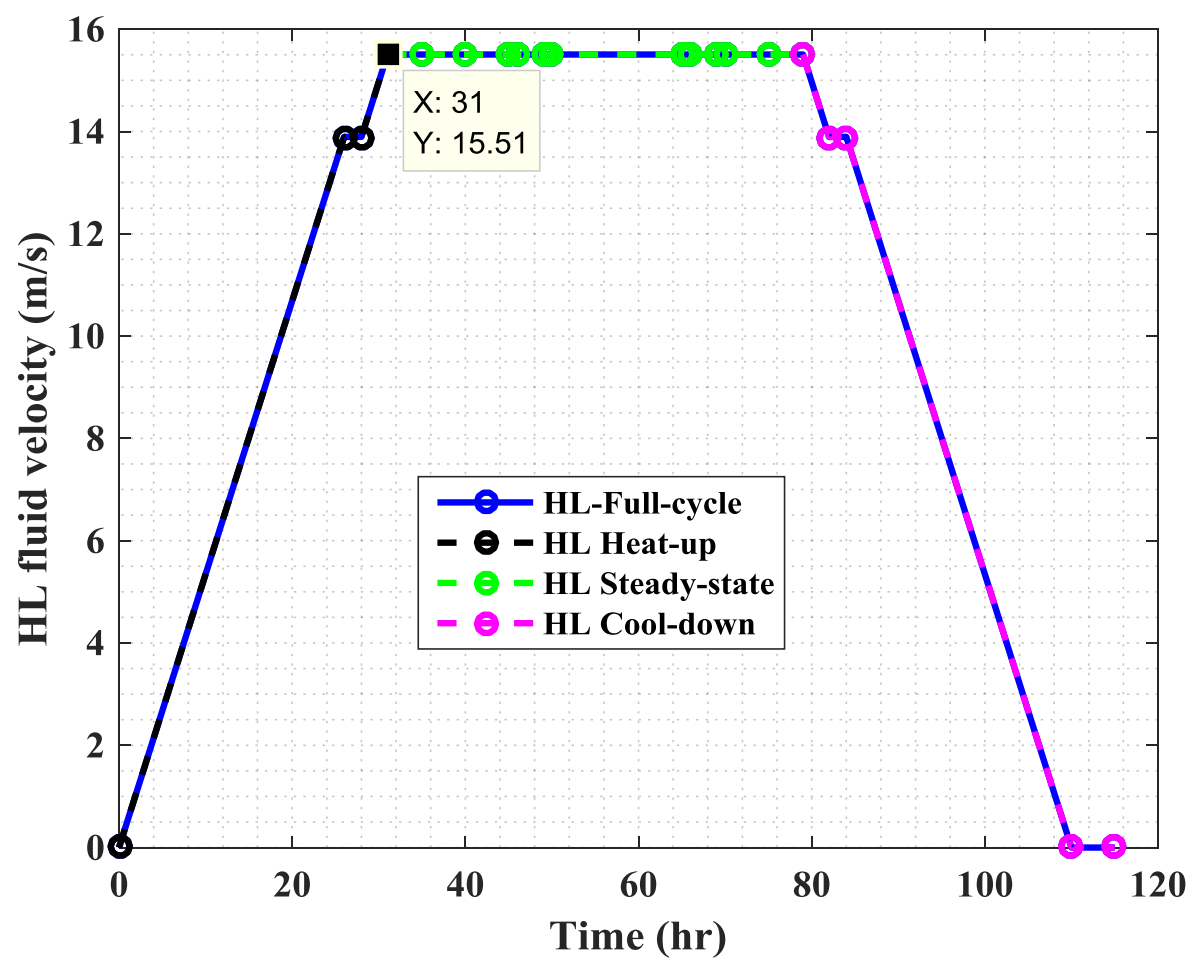

Figure 6. 4 Applied velocity boundary condition along a direction perpendicular to the axis of SL at SL-HL nozzle. 


\subsubsection{Material Properties}

The CFD model requires values of material properties (Eqs. 6.1-6.3) such as density ( $\rho$ ), dynamic viscosity $(\mu)$, specific heat capacity at constant pressure $\left(C_{p}\right)$, and thermal conductivity $\left(k_{f}\right)$. Note that we assume an incompressible flow condition with constant density. However, for the time-dependent velocity calculation, as shown in Figure 6.4, variable temperature densities were considered. Figure 6.5 shows the temperature-dependent density. Similarly Figures 6.6-76.6.8 show the temperature-dependent dynamic viscosity, specific heat capacity at constant pressure, and the thermal conductivity, respectively. The material properties were taken from [42].

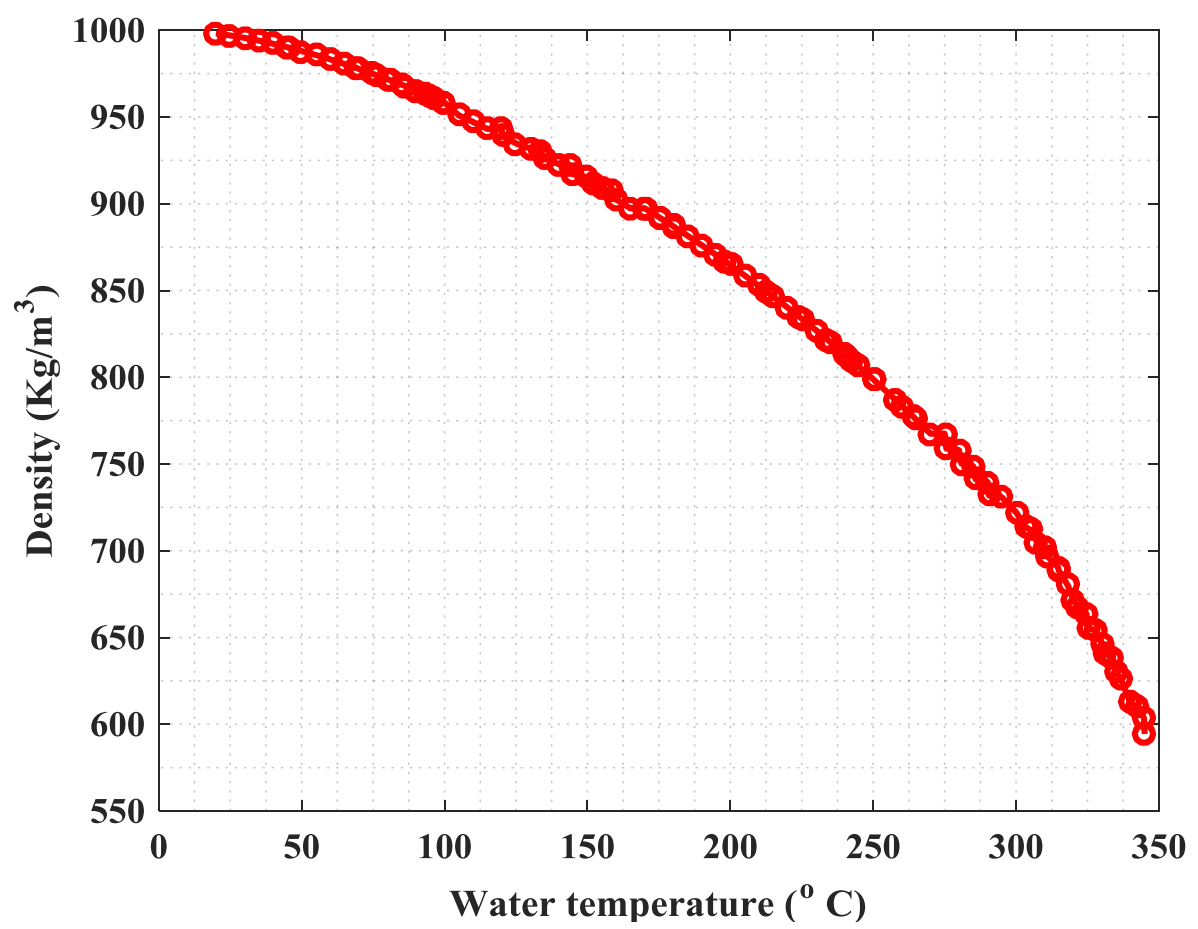

Figure 6. 5 Temperature-dependent density of water. 


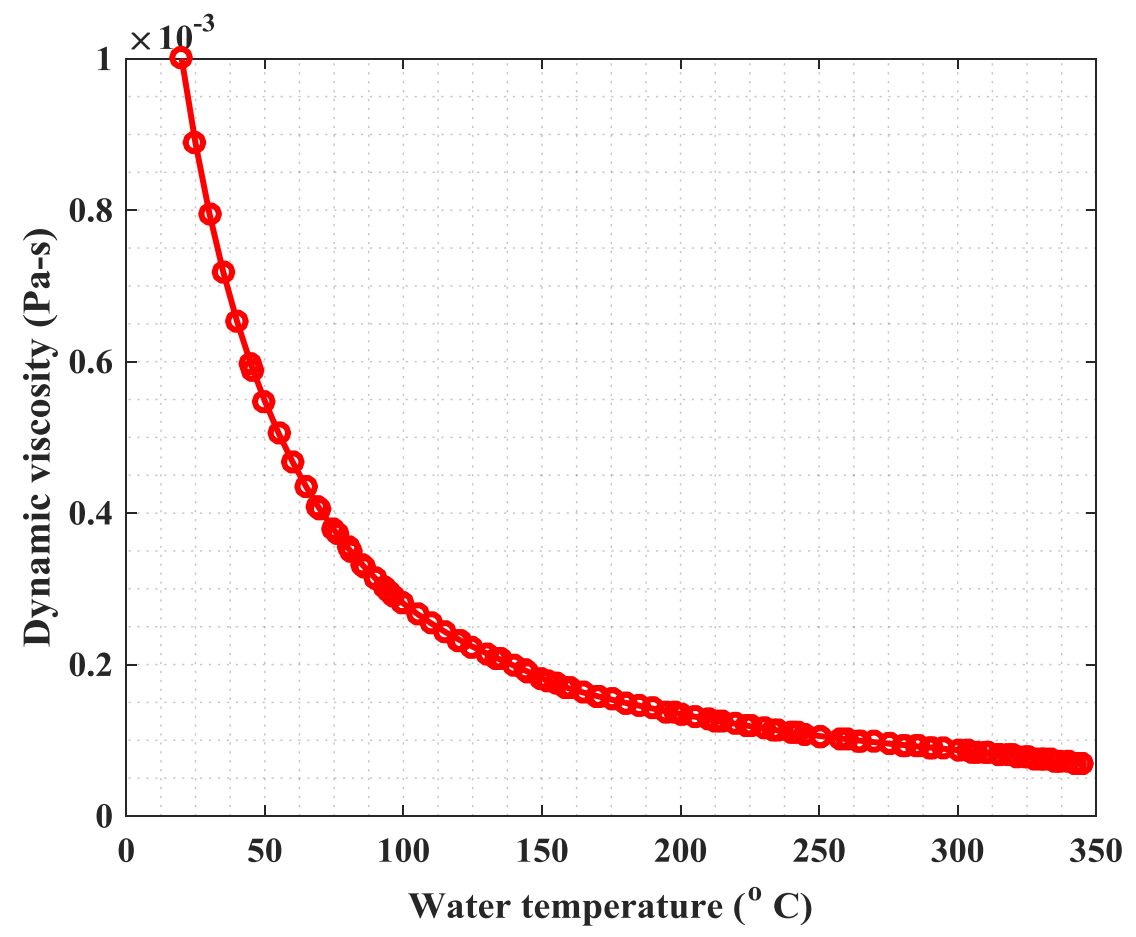

Figure 6. 6 Temperature-dependent dynamic viscosity of water.

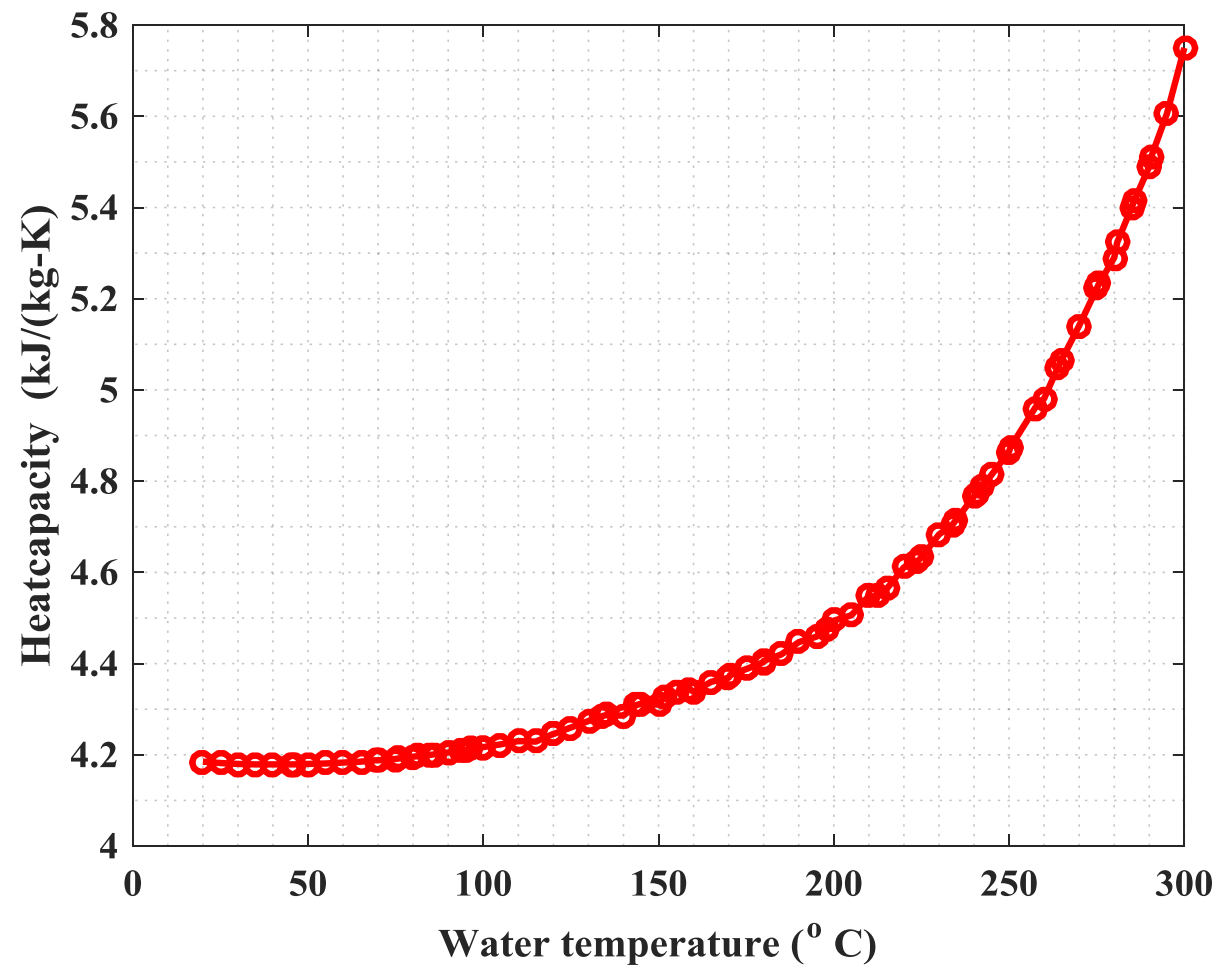

Figure 6. 7 Temperature-dependent heat capacity of water. 


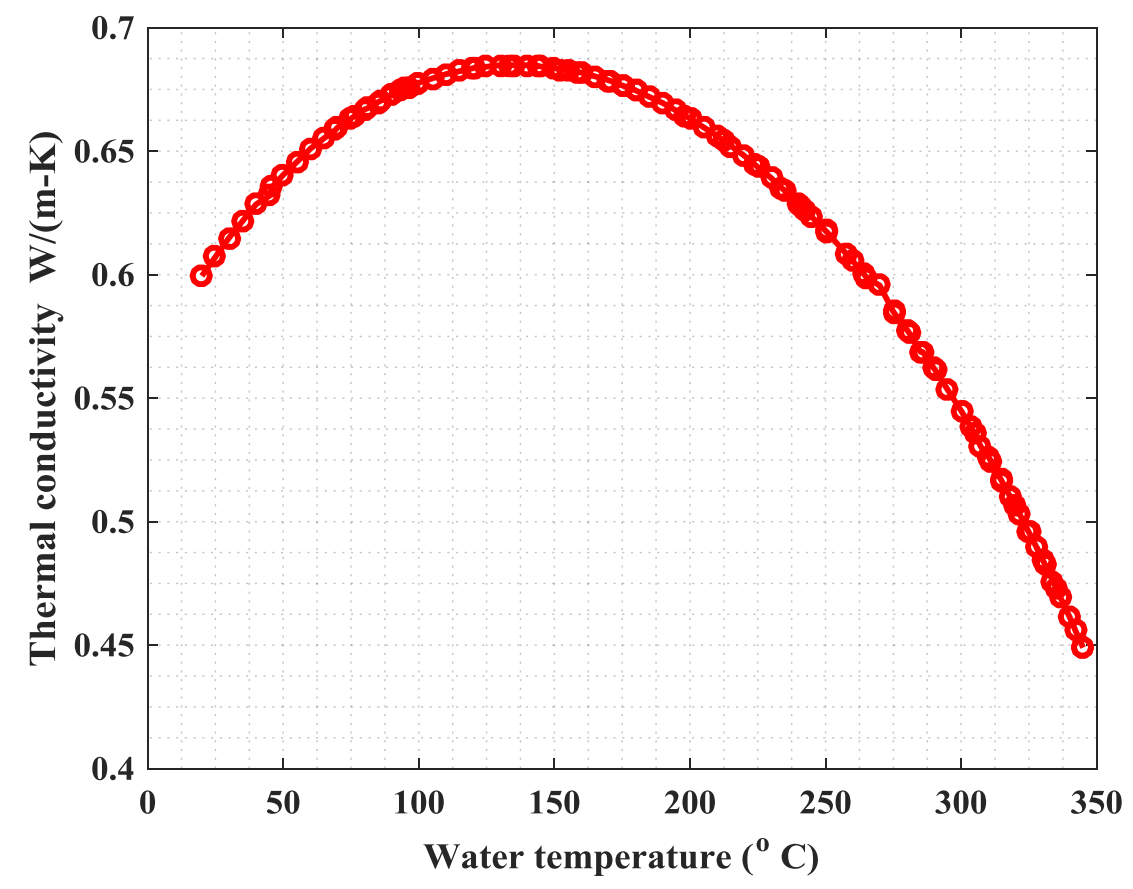

Figure 6. 8 Temperature-dependent thermal conductivity of water.

\subsection{Preliminary Results from CFD Simulation}

With the above-mentioned boundary condition a CFD simulation was conducted to estimate the temperature at the SL pipe ID (inner diameter). For simplicity, we only modeled the fluid and assumed the effect of the pipe wall would be insignificant, with the assumption of a perfectly insulated OD (outer diameter) surface on the SL pipe. Figure 6.9 shows the temperature history at a few representative nodes as highlighted in Figure 6.2. Figure 6.10 shows a magnified version of Figure 6.9, providing the temperature at the representative nodes (Figure 6.2) during a heat-up period. This figure indicates a temperature stratification (approximately of $30-35^{\circ} \mathrm{C}$ ) along the length of the SL. This temperature stratification is also visible in the temperature contour plot (at $\mathrm{t}=40 \times 10^{3} \mathrm{~s}$ or $11.1 \mathrm{hr}$ ) shown in Figure 6.11. Figure 6.12 shows a magnified version of Figure 6.9 during a steady-state power operation period. Figure 6.13 shows the temperature contour during a typical instance (at $\mathrm{t}=200 \times 10^{3} \mathrm{~s}$ or $55.55 \mathrm{hr}$ ) of steady-state power operation. Figures 6.12 and 6.13 show that the temperature in the SL is largely homogeneous and approximately the same as the temperature of the pressurizer. Figure 6.14 shows a magnified version of Figure 6.9 during a cool-down period, indicating the temperature variation at the representative nodes (Figure 6.2). Figure 6.15 shows an example temperature contour of the SL coolant wall at a typical instant $\left(\mathrm{t}=340 \times 10^{3} \mathrm{~s}\right.$ or $\left.94.4 \mathrm{hr}\right)$ during a cool-down period. Figures 6.14 and 15 show a thermal stratification (approximately of 30-35 ${ }^{\circ} \mathrm{C}$ ) along the length of SL during the cool-down operation. The above temperature stratification during heat-up and cool-down could lead to higher stress in SL pipe, which will be investigated in our future work. Also note that the CFD modeling work is ongoing to further improve the model, and the presented results are very preliminary. 


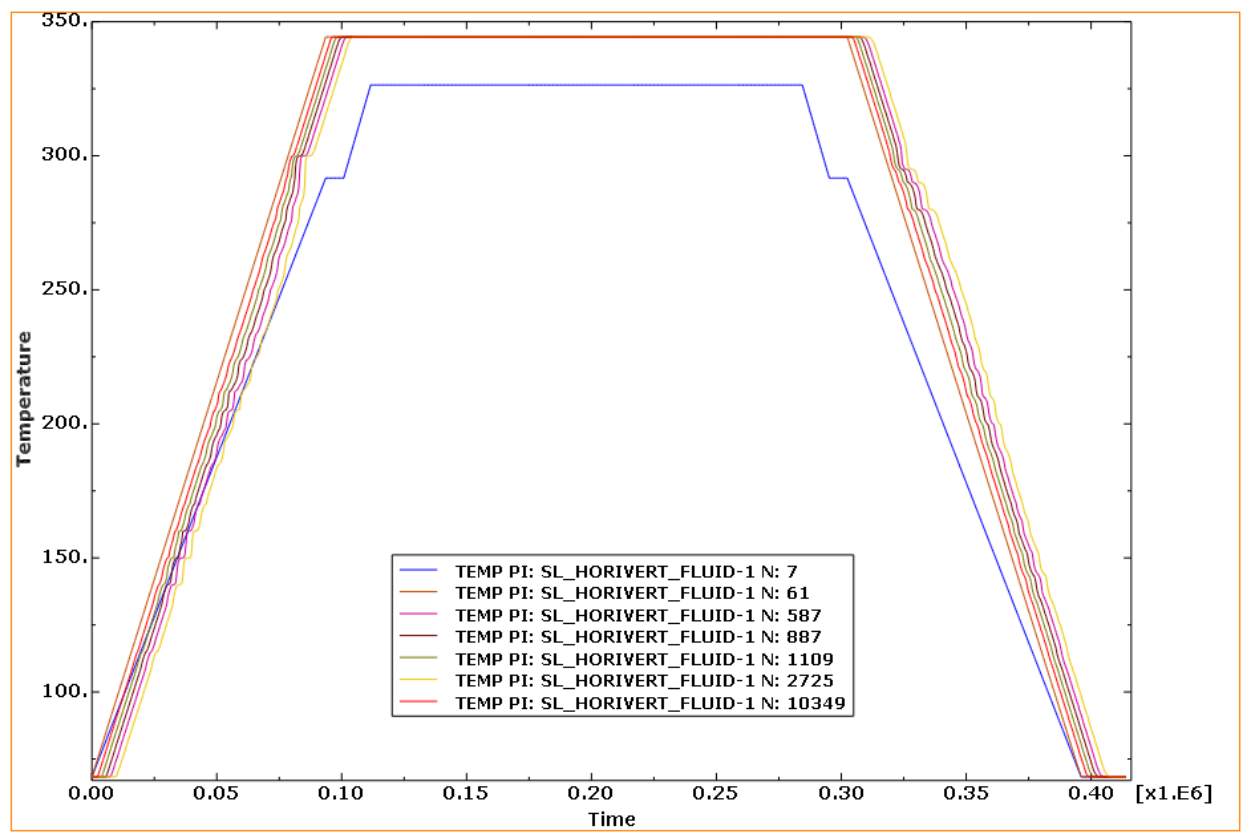

Figure 6. 9 Simulated temperature history at few representative nodes of SL (as highlighted in Figure 6.2)

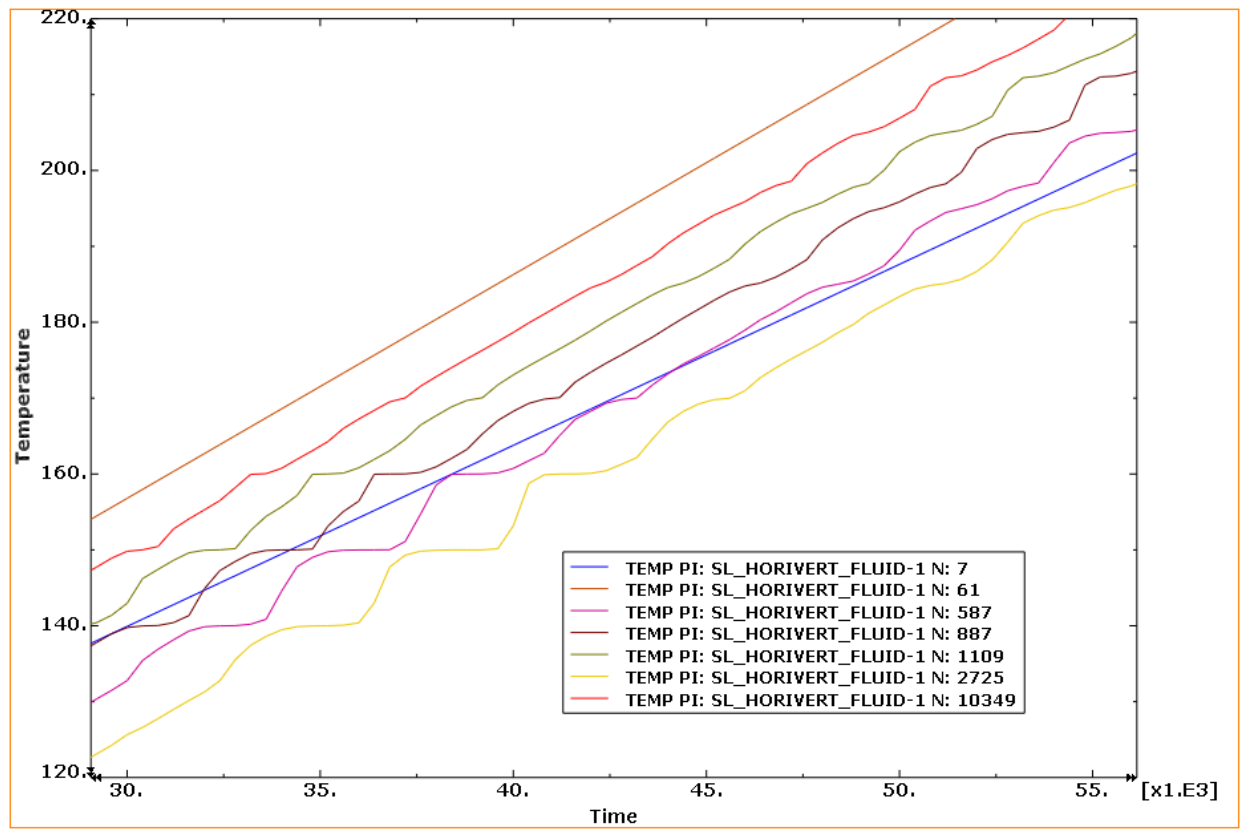

Figure 6. 10 Magnified $\left(t=30 \times 10^{3} \mathrm{~s}\right.$ to $\left.55 \times 10^{3} \mathrm{~s}\right)$ version of Figure 6.9. 


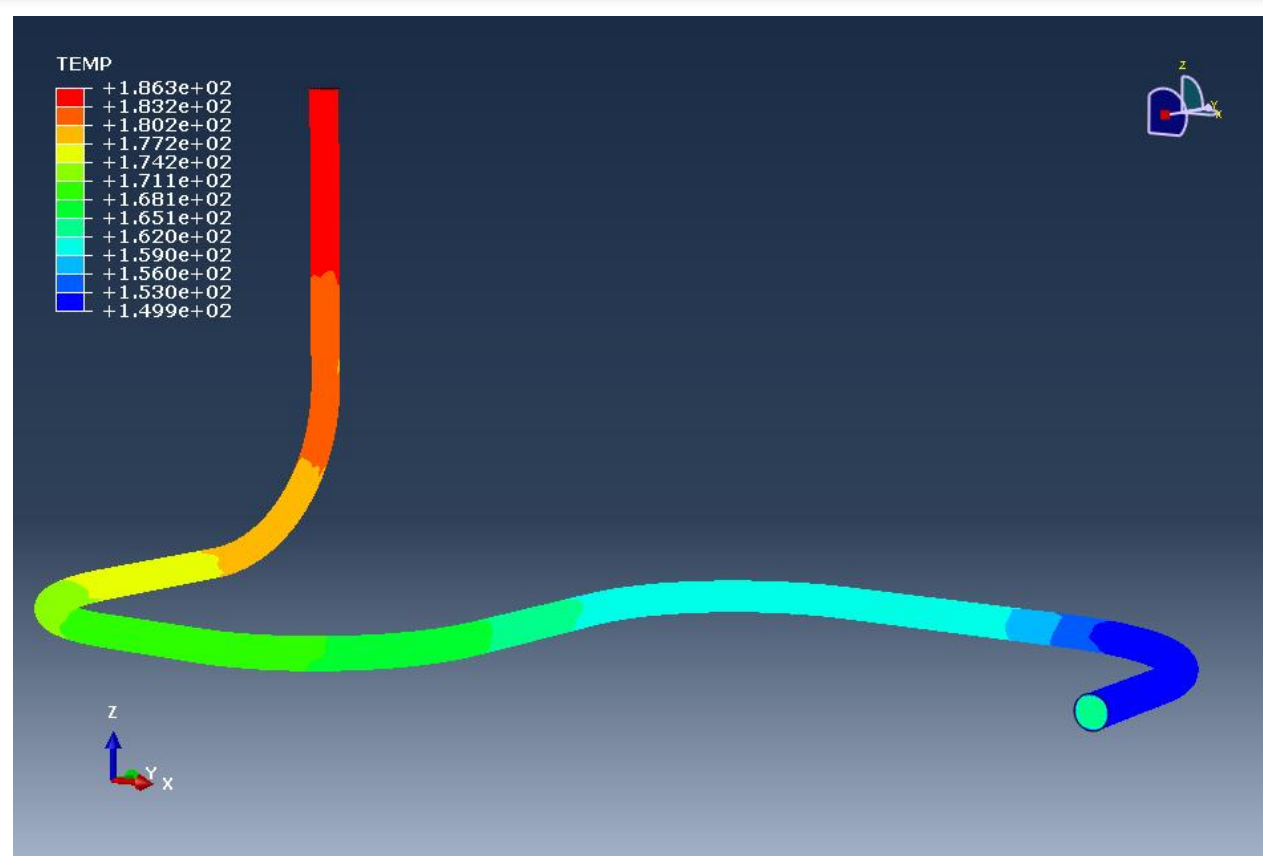

Figure 6. 11 Temperature contour at a typical instant during heat-up (at $\mathrm{t}=40 \times 10^{3} \mathrm{~s}$ or $11.1 \mathrm{hr}$ ).

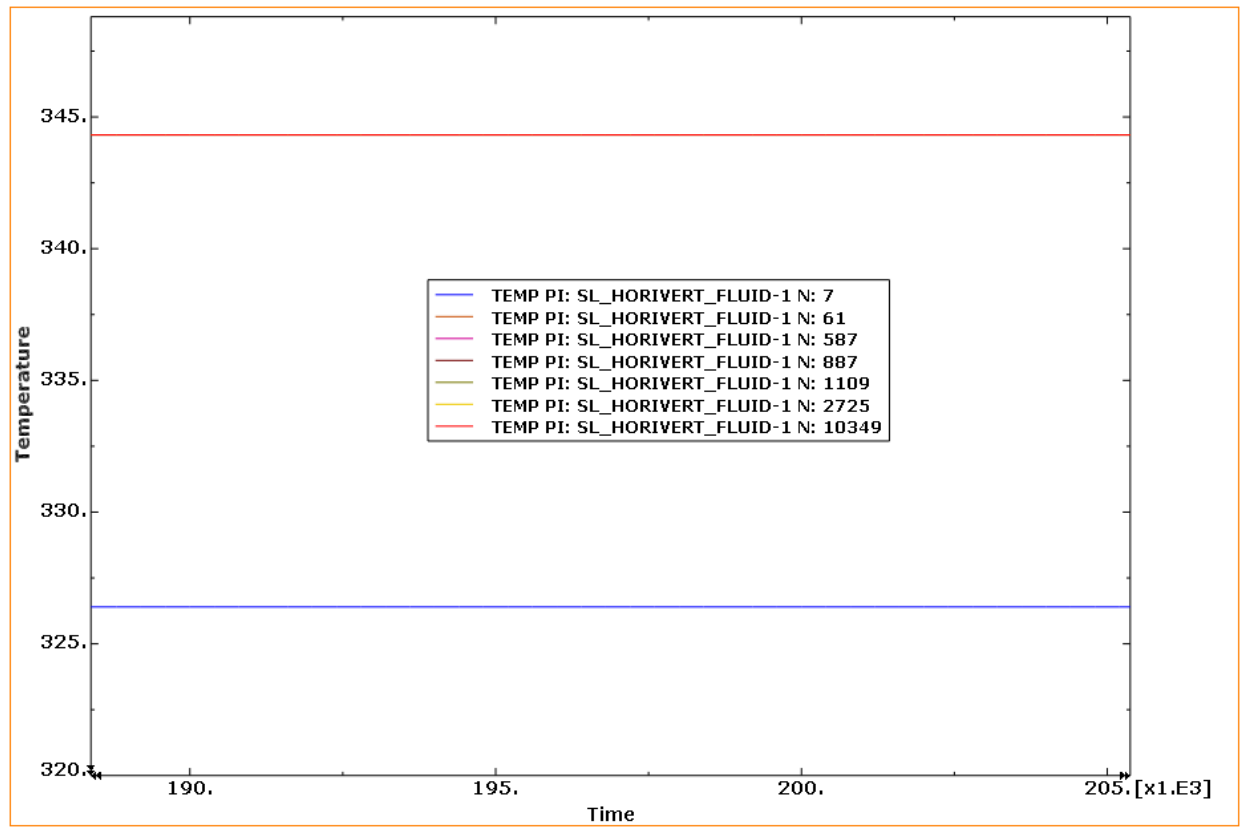

Figure 6. 12 Magnified $\left(\mathrm{t}=188 \times 10^{3}\right.$ to $\left.205 \times 10^{3} \mathrm{~s}\right)$ version of Figure 6.9. 


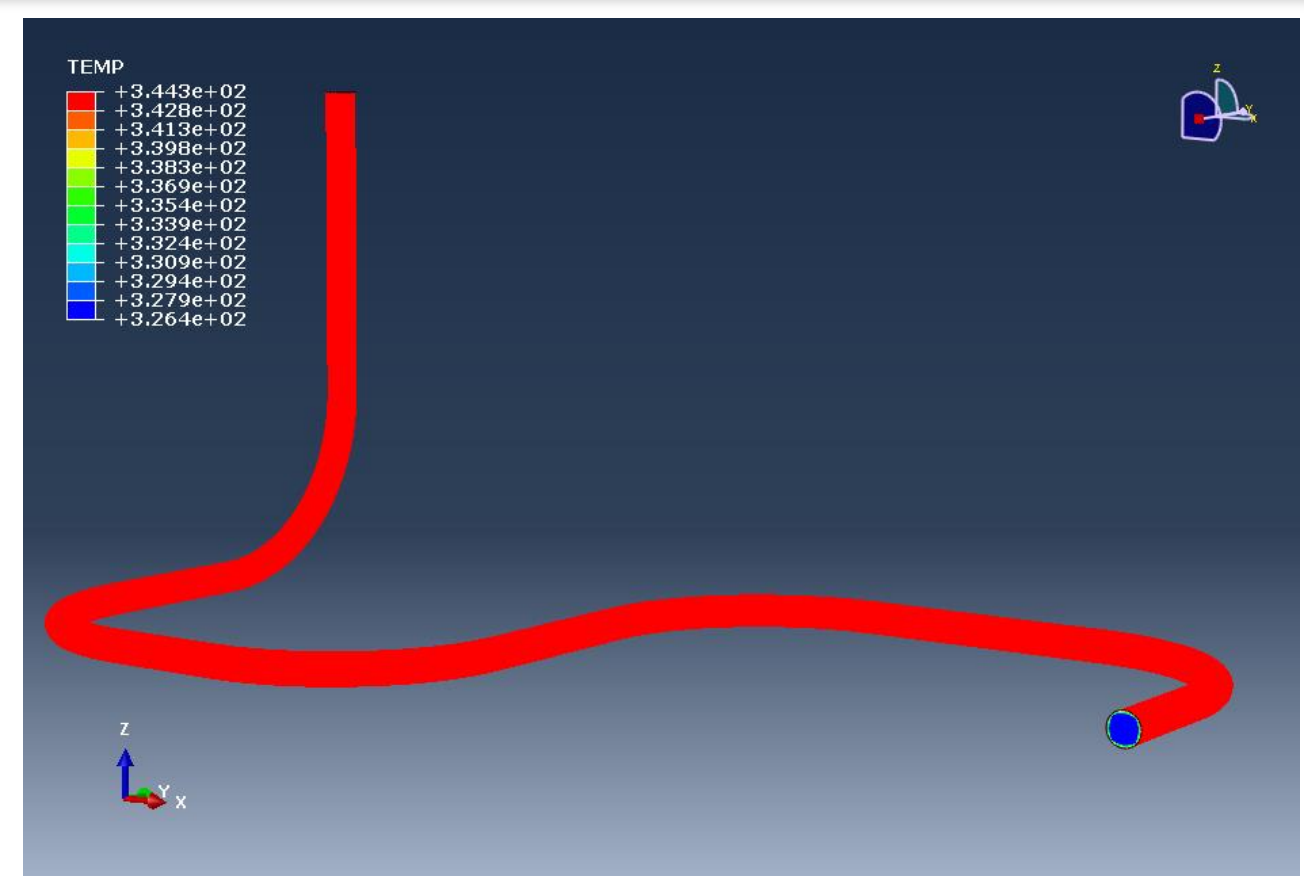

Figure 6. 13 Temperature contour at a typical instant during steady-state power operation $($ at $\mathrm{t}=$ $200 \times 10^{3} \mathrm{~s}$ or $55.55 \mathrm{hr}$ ).

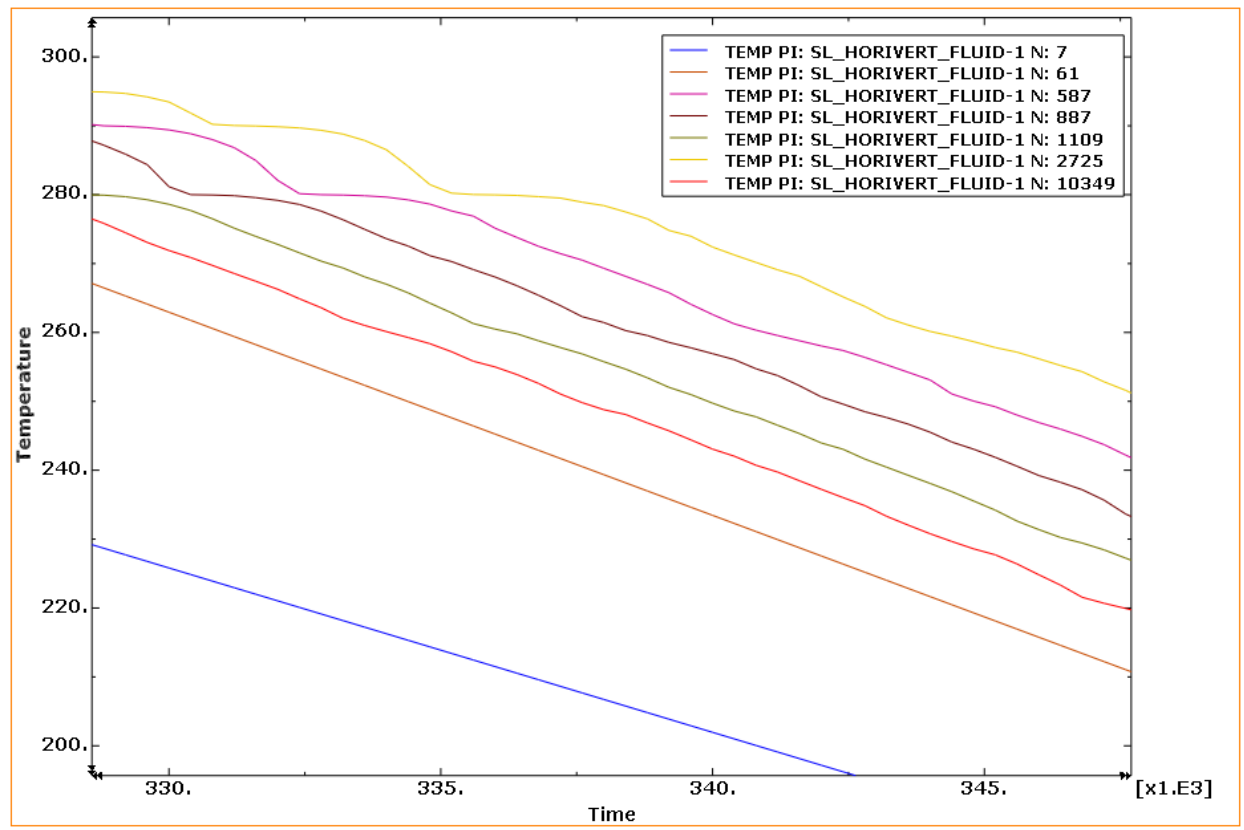

Figure 6. 14 Magnified $\left(\mathrm{t}=328 \times 10^{3} \mathrm{~s}\right.$ to $\left.347.5 \times 10^{3} \mathrm{~s}\right)$ version of Figure 6.9. 


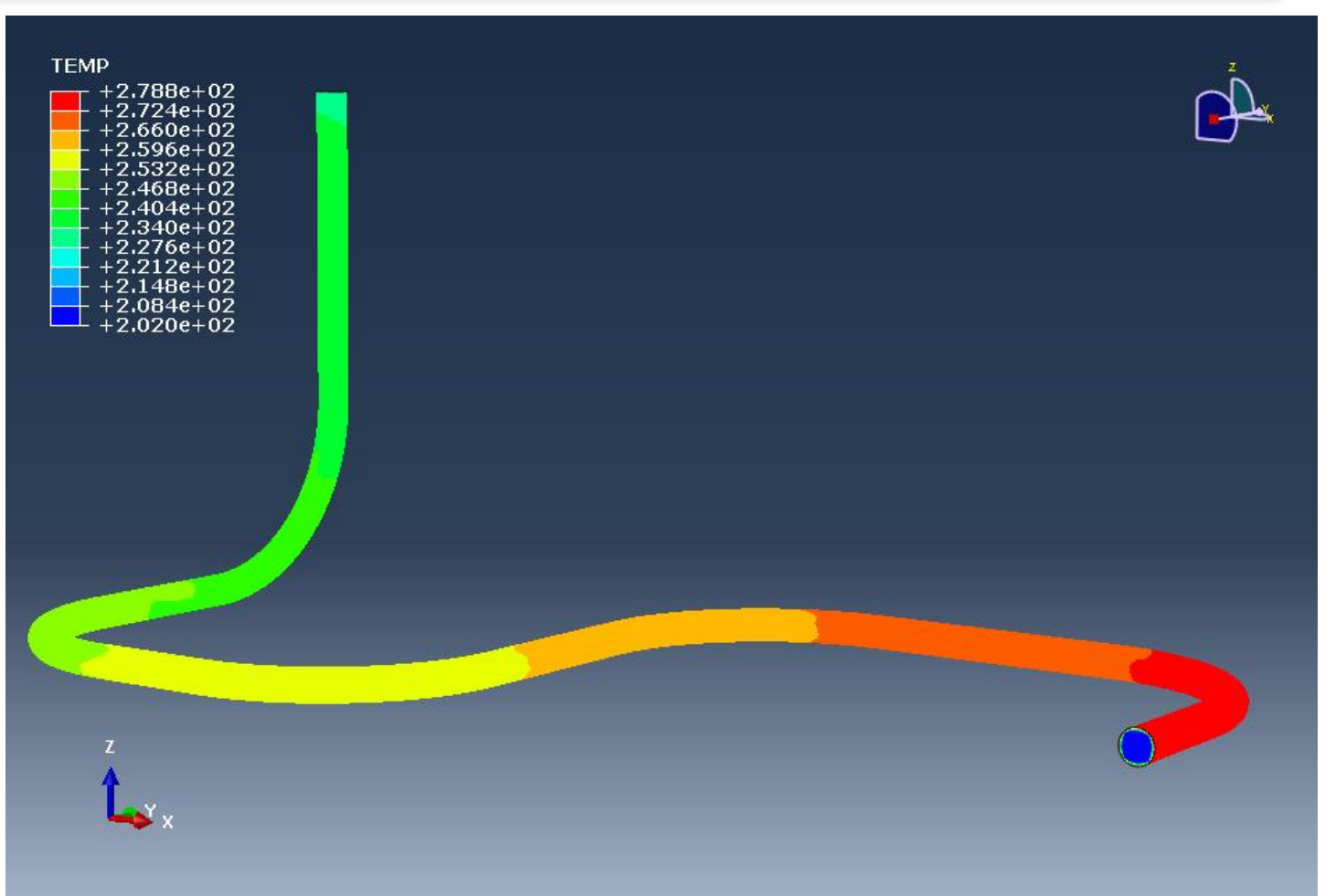

Figure 6. 15 Temperature contour at a typical instant during cool-down (at t $=340 \times 10^{3} \mathrm{~s}$ or $94.4 \mathrm{hr}$ ). 


\section{Summary and Future Study}

In this work, $316 \mathrm{SS}$ laboratory-scale specimens subjected to strain-controlled cyclic loading with constant, variable, and random amplitude were modeled with the Argonne-developed evolutionary cyclic plasticity model. Results from 1D-analytical modeling and 3D-FE modeling simulations of the entire life of the specimens are presented and compared with experimentally observed data. A user material subroutine was developed to enable the implementation of the cyclic plasticity model into a commercial FE code, ABAQUS. Two modeling frameworks are discussed: a time-based approach to model constantand variable-amplitude fatigue tests and a more generalized and robust approach based on APSE to predict material behavior under any type of loading inputs, including random-amplitude cyclic loading. Based on the results from 1D-analytical and 3D-FE modeling of fatigue specimens, the following major conclusions are drawn:

1. The simulated results demonstrate that the evolutionary cyclic plasticity model can mechanistically capture all the important stages of material behavior (initial hardening, softening, stabilized cycles, and finally rapid crack propagation followed by failure) during the entire fatigue life of the specimens with great accuracy.

2. Lives predicted by time-based $1 \mathrm{D}$-analytical and 3D-FE modeling are almost $100 \%$ accurate for all the constant- and variable-amplitude fatigue test specimens.

3. The APSE-based analytical modeling of the constant, variable, and random amplitude predicts the lives of the specimens with more than $90 \%$ accuracy. In addition, the accuracy in life prediction is between $70 \%$ and $90 \%$ from 3D-FE simulation. The reason for the less accurate estimation with 3DFE modeling and means to improve the accuracy are discussed from the FE perspective.

The major aim of this research project is to develop a fully mechanistic fatigue life evaluation technique for nuclear reactor components and thereby greatly reduce the uncertainty in the estimated fatigue life using $\mathrm{S} \sim \mathrm{N}$ curve-based empirical methods. The authors believe that the research work presented in this report is a big step toward that major goal. Mechanistic simulation would allow engineers to validate their design under real-loading conditions with greater confidence. As the computational hardware and software capacity rapidly advancing, component and system-level simulation for thousands of fatigue cycles is not impossible now. The final aim of this research is to perform mechanistic based fatigue simulation of the nuclear reactor's entire cooling system utilizing one of the Argonne's supercomputer facilities e.g. Mira [43] or Aurora [44].

This report also presents some preliminary results from component-level computer modeling of a specific reactor component, namely, 316 SS surge line pipe. Results from CFD modeling to evaluate temperature boundary conditions and thermal stratification of the surge line pipes are presented and discussed. 
Suggested future work includes the following:

1. Perform stress-controlled fatigue experiments of 316 SS under constant, variable, and random amplitude loading.

2. Simulate the stress-controlled fatigue experiments through 1D-analytical and 3D-FE modeling.

Perform thermal-mechanical stress analysis of the reactor surge line pipe through coupling the evolutionary cyclic plasticity model based FE framework with the CFD model for a fully mechanistic fatigue life evaluation. 


\section{References}

1. The American Society of Mechanical Engineers (2004) ASME Boiler and Pressure Vessel Code Section VIII, Division 2, Alternative Rules, Rules for Construction of Nuclear Power Plant Components.

2. O.K. Chopra, W.J. Shack (2007) Effect of LWR Coolant Environments on the Fatigue Life of Reactor Materials, U.S. Nuclear Regulatory Commission, Report no. NUREG/CR-6909.

3. The American Society of Mechanical Engineers (2013) ASME Boiler and Pressure Vessel Code, Section III, Division1, Rules for Construction of Nuclear Facility Components.

4. BSi (2012) BS EN 13445-3:2009+A1:2012, Unfired Pressure Vessels. Part 3: Design.

5. BSi (2012) PD 5500:2012, Specification for Unfired Fusion Welded Pressure Vessels.

6. EDF Energy Generation (2003) R5-Assessment Procedure for the High Temperature Response of Structures, Issue 3, Revision 1.

7. AFCEN (2007) RCC-M- Edition 2007 -Addendum December 2008: Design and Construction Rules for Mechanical Components of PWR Nuclear Islands.

8. N. Platts, D.R. Tice, J. Nicholls (2015) Study of Fatigue Initiation of Austenitic Stainless Steel in a High Temperature Water Environment and in Air Using Blunt Notch Compact Tension Specimens, ASME 2015 Pressure Vessels and Piping Conference, Paper no. PVP2015-45844.

9. J. Shi, L. Wei, C. Faidy, A. Wasylyk, N. Prinja (2016) A Comparison of Different Design Codes on Fatigue Life Assessment Methods, ASME 2016 Pressure Vessels and Piping Conference, Paper no. PVP2016-63040.

10. J. Shit, S. Dhar, S. Acharyya. (2013) Modeling and Finite Element Simulation of Low Cycle Fatigue Behaviour of 316 SS, Procedia Engineering 55, pp. 774-779.

11. Y.F. Dafalias, E.P. Popov (1975) A Model of Nonlinearly Hardening Materials for Complex Loading, Acta mechanica 21, pp. 173-192.

12. P.J. Armstrong, C.O. Frederick. (1966) A Mathematical Representation of the Multiaxial Bauschinger Effect, Central Electricity Generating Board and Berkeley Nuclear Laboratories, Research \& Development Department.

13. J.L. Chaboche (1986) Time-independent Constitutive Theories for Cyclic Plasticity, International Journal of Plasticity 2, pp. 149-188.

14. J.L. Chaboche (1991) On Some Modifications of Kinematic Hardening to Improve the Description of Ratchetting Effects, International Journal of Plasticity 7, pp. 661-678.

15. J.L. Chaboche, G. Rousselier. (1983) On the Plasticity and viscoplasticity Constitutive Equationspart II: Application of Internal Variable Concepts to the 316 Stainless Steel, Journal of Pressure Vessel Technology 105, pp. 159-164.

16. N.N. Ohno Y.Y. Takahashi, K.K. Kuwabara (1989) Constitutive Modeling of Anisothermal Cyclic Plasticity of 304 Stainless Steel, Journal of Engineering and Materials Technology 111, pp.106-114.

17. G. Kang (2006) Finite Element Implementation of Visco-plastic Constitutive Model with Strainrange-dependent Cyclic Hardening, International Journal for Numerical Methods in Biomedical Engineering 22, pp. 137-153.

18. G. Kang, N. Ohno, A. Nebu (2003) Constitutive Modeling of Strain Range Dependent Cyclic Hardening, International journal of plasticity 19, pp. 1801-1819.

19. N. Ohno, J.D. Wang (1993) Kinematic Hardening Rules with Critical State of Dynamic Recovery, part I: Formulation and Basic Features for Ratchetting Behavior, International journal of plasticity 9 , 375-390. 
20. S.M Syed, T. Hassan, E. Corona. (2008) Evaluation of Cyclic Plasticity Models in Ratcheting Simulation of Straight Pipes under Cyclic Bending and Steady Internal Pressure, International Journal of Plasticity 24, pp. 1756-1791.

21. G. Sumit, S.K. Gupta, S. Sivaprasad, S. Tarafder, V. Bhasin, K.K. Vaze, A.K. Ghosh (2013) Low Cycle Fatigue and Cyclic Plasticity Behavior of Indian PHWR/AHWR Primary Piping Material, Procedia Engineering 55, pp. 136-143.

22. S. Mohanty, W.K. Soppet, S. Majumdar, K. Natesan, (2015) Effect of Pressurized Water Reactor Environment on Material Parameters of 316 Stainless Steel: A Cyclic Plasticity Based Evolutionary Material Modeling Approach, ASME 2015 Pressure Vessels and Piping Conference, Paper no. PVP2015-45701.

23. S. Mohanty, W.K. Soppet, S. Majumdar, K. Natesan, (2016) Chaboche-Based Cyclic Material Hardening Models for 316 SS-316 SS Weld under In-air and Pressurized Water Reactor Water Conditions, Nuclear Engineering and Design 305, pp 524-530.

24. S. Mohanty, W.K. Soppet, S. Majumdar, S., K. Natesan, K. (2015) Tensile and Fatigue Testing and Material Hardening Model Development for 508 LAS Base Metal and 316 SS Similar Metal Weld under In-air and PWR Primary Loop Water Conditions, Argonne National Laboratory, Report no. ANL/LWRS-15/02. (https://www.osti.gov/scitech/biblio/1224989).

25. S. Mohanty, W.K. Soppet, S. Majumdar, S., K. Natesan, K. (2016) In-Air and Pressurized Water Reactor Environment Fatigue Experiments of 316 Stainless Steel to Study the Effect of Environment on Cyclic Hardening, Journal of Nuclear Materials 473, pp. 290-299.

26. S. Mohanty, B. Barua, W.K. Soppet, S. Majumdar, S., K. Natesan, K. (2016) Study the Cyclic Plasticity Behavior of 508 LAS under Constant, Variable and Grid-Load-Following Loading Cycles for Fatigue Evaluation of PWR Components, Argonne National Laboratory, Report no. ANL/LWRS16/03. (https://www.osti.gov/scitech/biblio/1331616).

27. M.L. Wilkins (1963) Calculation of elastic-plastic flow, California University Livermore Radiation Lab, Report no. UCRL-7322.

28. S.K. Koh (2002) Fatigue Damage Evaluation of a High Pressure Tube Steel using Cyclic Strain Energy Density, International Journal of Pressure Vessels and Piping, 79, pp. 791-798.

29. X. Lin, G. Halcheng (1998) Plastic Energy Dissipation iVJodel for Lifetime Prediction of Zirconium and Zircaloy-4 Fatigued at RT and $400^{\circ} \mathrm{C}$, Journal of Engineering Materials and Technology 120, pp. 114-118.

30. T. Lagoda (2001) Energy Models for Fatigue Life Estimation under Uniaxial Random Loading. Part I: The Model Elaboration, International Journal of Fatigue 23, pp. 467-480.

31. B. Fekete (2015) New Energy-based Low Cycle Fatigue Model for Reactor Steels, Materials \& Design, 79, pp. 42-52.

32. A.N. Kadhim, M. Mustafa, A. Varvani-Farahani (2015) Fatigue Life Prediction of Low-alloy Steel Samples Undergoing Uniaxial Random Block Loading Histories based on Different Energy-based Damage Descriptions, Fatigue \& Fracture of Engineering Materials \& Structures, 38, pp. 69-79.

33. J. Morrow (1965) Cyclic Plastic Strain Energy and Fatigue of Metals, ASTM International, Internal Friction, Damping, and Cyclic plasticity.

34. G.R. (1966) The Energy Required for Fatigue (Plastic Strain Hysteresis Energy Required for Fatigue in Ferrous and Nonferrous Metals, Journal of materials, 1, pp. 3-18.

35. B.N. Leis (1977) An Energy-based Fatigue and Creep-fatigue Damage Parameter, Journal of Pressure Vessel Technology, 99, pp. 524-533.

36. Systémes, Dassault. (2016) "Abaqus Theory Guide". 
37. S. Mohanty, W.K. Soppet, S. Majumdar, S., K. Natesan, K. (2014). Environmental Effect on Evolutionary Cyclic Plasticity Material Parameters of 316 Stainless Steel: An Experimental \& Material Modeling Approach, Argonne National Laboratory, Report no. ANL/LWRS-14/01. (https://www.osti.gov/scitech/biblio/1168233).

38. ABAQUS user manual (2011) and (2016).

39. Westinghouse Technology Systems Manual, Section 19.0 - Plant Operation (Source: https://www.nrc.gov/docs/ML1122/ML11223A342.pdf).

40. G. Meyer, E. Stokke (1997) Description of Sizewell B nuclear power plant. NKS (Source: http://www.iaea.org/inis/collection/NCLCollectionStore/_Public/29/010/29010110.pdf).

41. V. N. Shah, P.E. MacDonald (1993) Aging and life extension of major light water reactor components, Elsevier Science Publishers B.V., Amsterdam, The Netherland, ISBN: 0444894489.

42. V.P. Bobkov, L.R. Fokin, E.E. Petrov, V.V. Popov, V.N. Rumiantsev, A.I. Savvatimsky, (2008) Thermophysical Properties of Materials for Nuclear Engineering: A Tutorial and Collection of Data. IAEA, Vienna. (Source: http://www-pub.iaea.org/books/iaeabooks/7965/ThermophysicalProperties-of-Materials-for-Nuclear-Engineering-A-Tutorial-and-Collection-of-Data).

43. https://www.alcf.anl.gov/mira

44. http://aurora.alcf.anl.gov/ 


\section{Appendices}

The appendices contain additional experimental and material modeling results of the tensile (ET-T04) and fatigue (constant-amplitude: ET-F06, ET-F41; variable-amplitude: ET-F38; and random-amplitude: ET-F40) tests discussed in this report. The supplementary experimental results include observed actuator position and crosshead stroke data. The supplementary material modeling results for the tensile test (ETT04) include estimated material parameters considering yield stress at elastic limit and at $0.05 \%, 0.1 \%$, and $0.2 \%$ offset strain limit. Tensile test based (time-independent or fixed) both linear (C1 only) and nonlinear (C1 and $\gamma 1)$ kinematic hardening parameters are provided. For fatigue test based material properties (time/cycle/block-dependent), nonlinear kinematic hardening parameters (C1 and $\gamma 1)$ were estimated considering yield stress at elastic limit and $0.05 \%$ offset strain limit, while linear (C1 only) kinematic hardening parameters were estimated considering yield stress at $0.2 \%$ offset strain limit. In this work, however, nonlinear kinematic hardening parameters ( $\mathrm{C} 1$ and $\gamma 1)$ considering yield stress at the elastic limit were only used for both analytical and 3D-FE modeling. Other material parameters are provided in the appendices in case readers are interested in using those; however, authors do not have data on accuracy of the predictions using those parameters.

\section{Appendix-3A: Supplementary Results for ET-T04 Tensile Test}

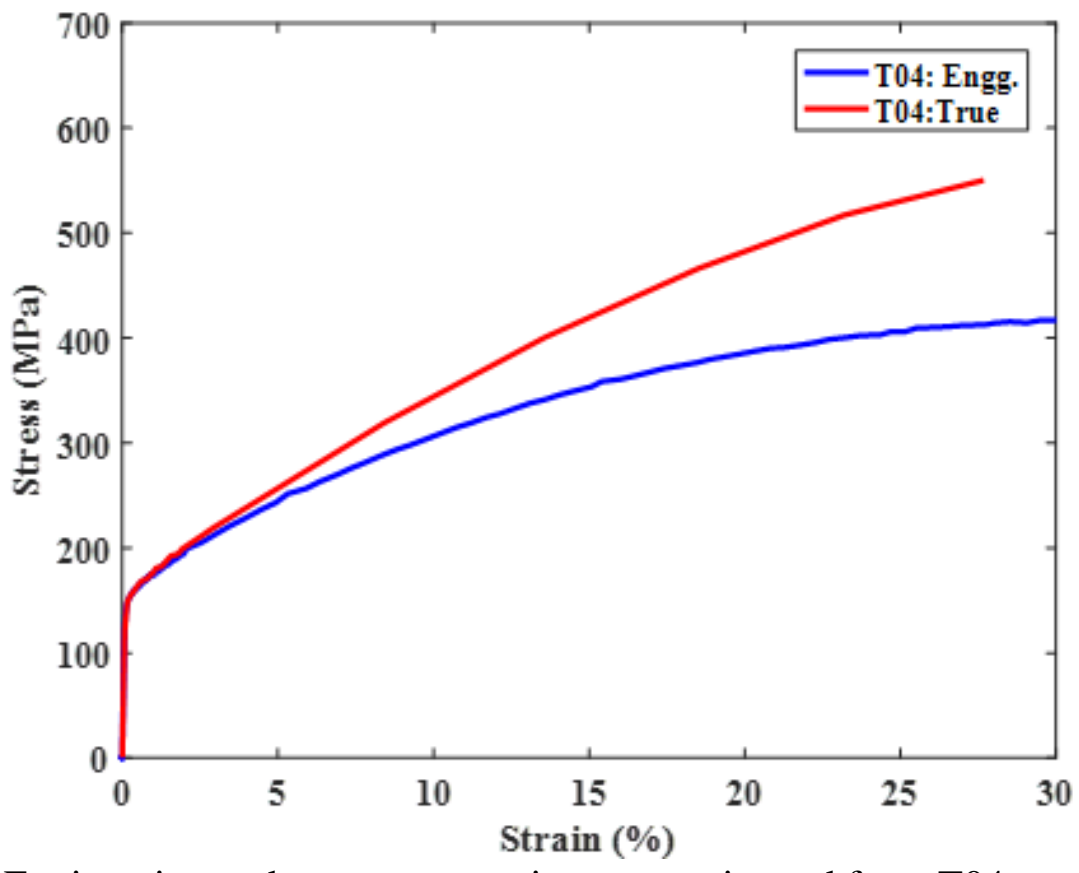

Figure 3A. 1 Engineering and true stress-strain curve estimated from T04 tensile test data. 


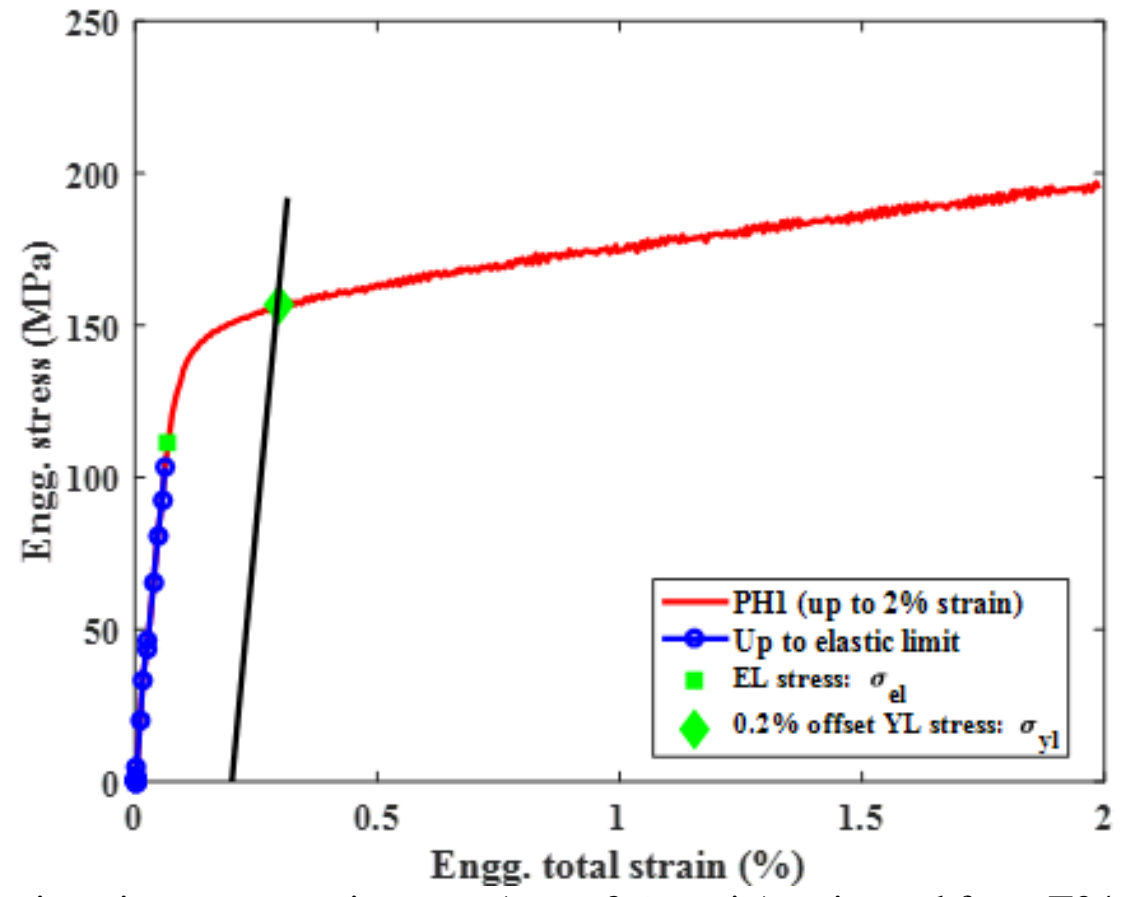

Figure 3A. 2 Engineering stress-strain curve (up to $2 \%$ strain) estimated from T04 tensile test data showing assumed elastic limit (EL) and estimated $0.2 \%$ offset yield limit (YL).

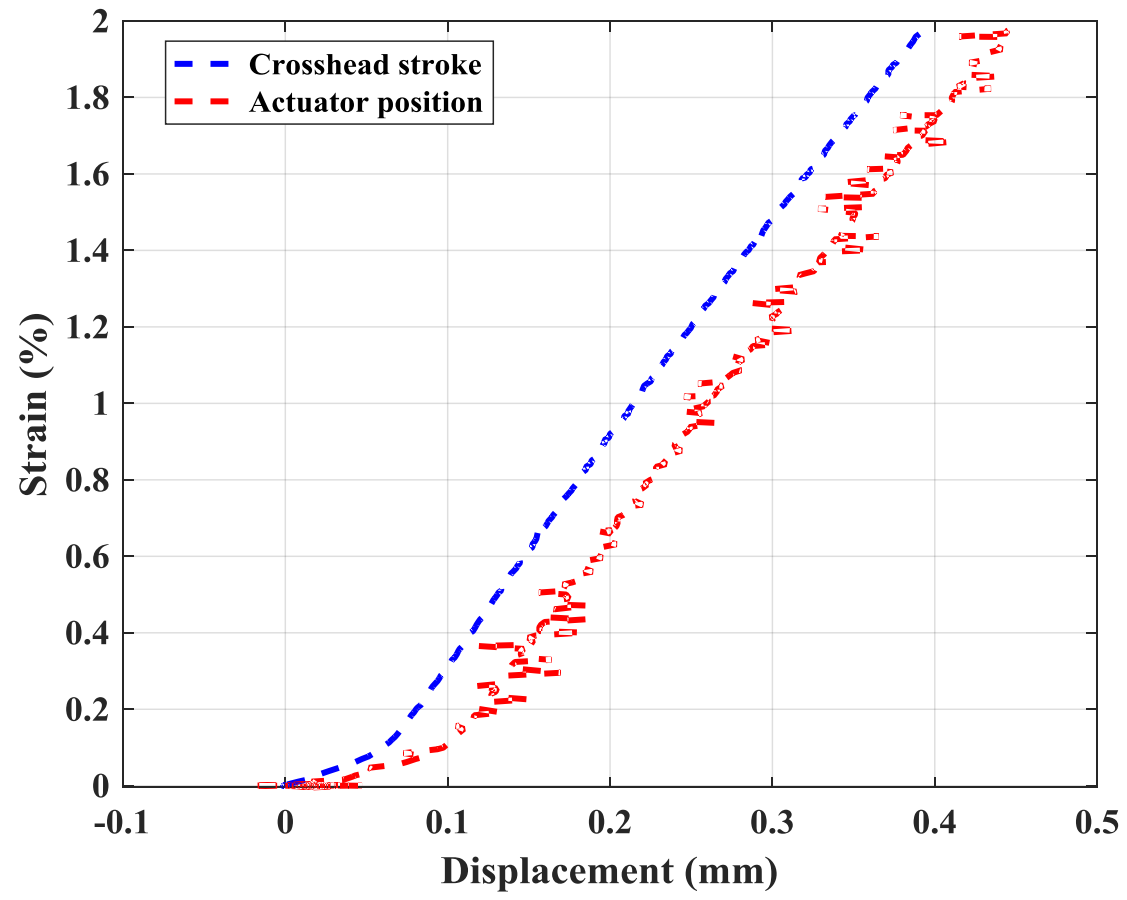

Figure 3A. 3 Frame crosshead displacement (stroke) and actuator position versus strain (up to $2 \%$ strain), estimated from ET-T04 tensile test data. These types of results will be used for selecting test parameters for stroke control and position control fatigue tests. 


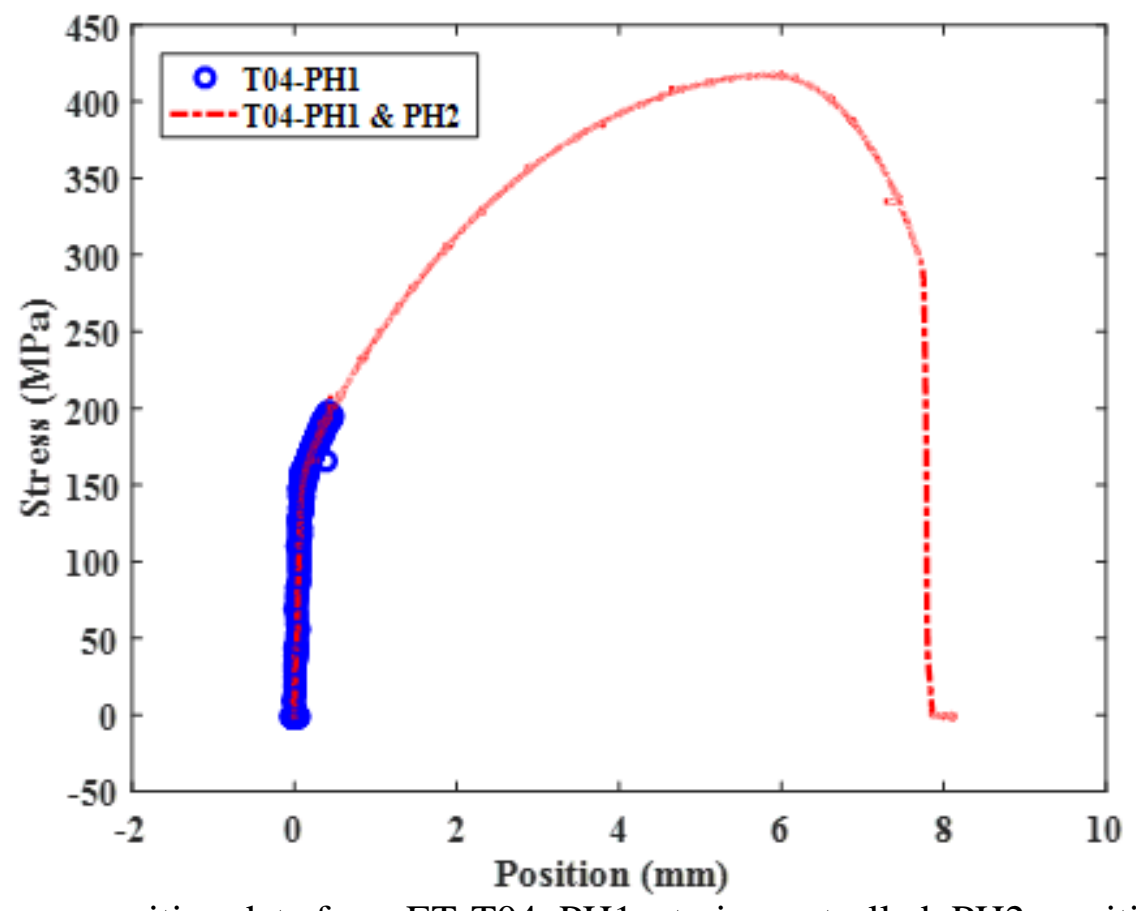

Figure 3A. 4 Stress vs position data from ET-T04. PH1: strain-controlled, PH2: position-control. Strain was predicted from position in $\mathrm{PH} 2$. This type of result will be used to estimate ratcheting strain from a stress-controlled fatigue test.

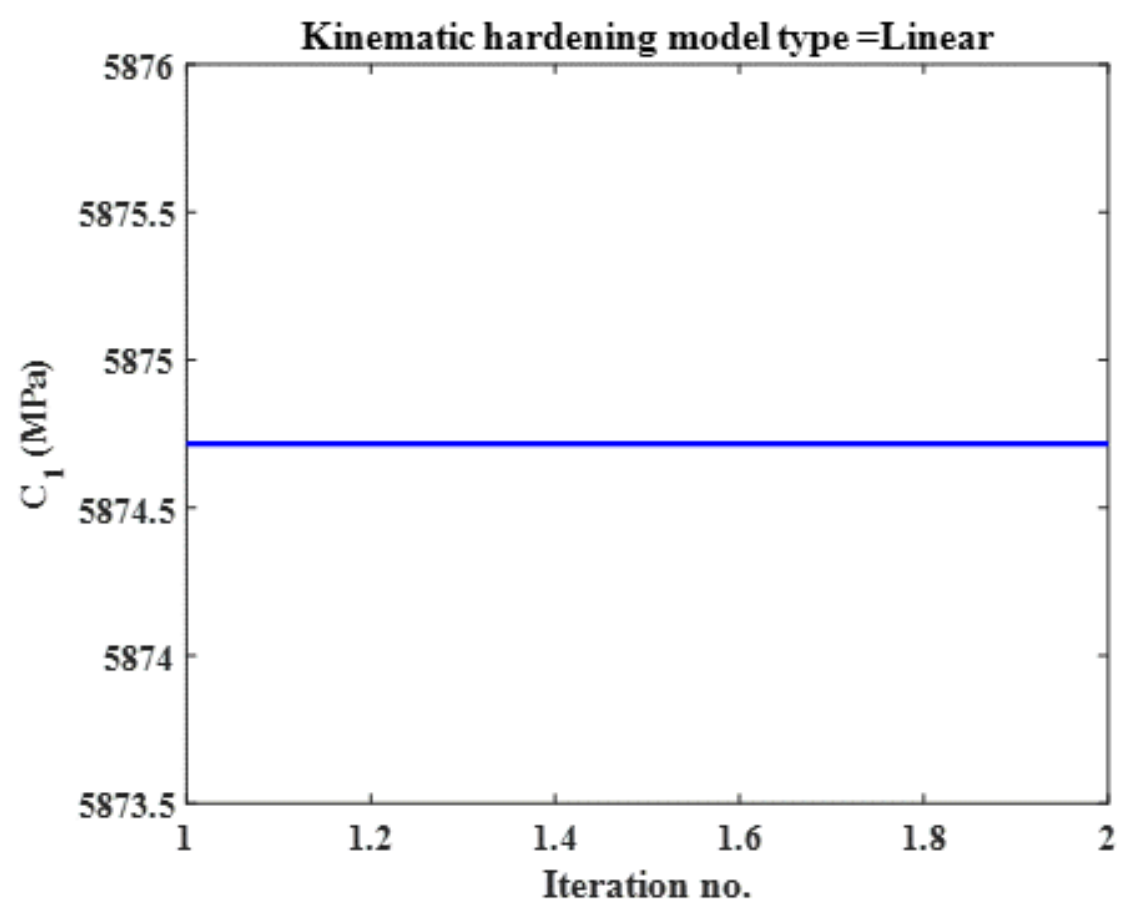

Figure 3A. 5 Linear kinematic hardening parameter $\mathrm{C} 1$ with respect to number of iteration during parameter estimation using gradient based optimization scheme and using ET-T04 stress-strain data (from elastic limit to $2 \%$ true total strain). 


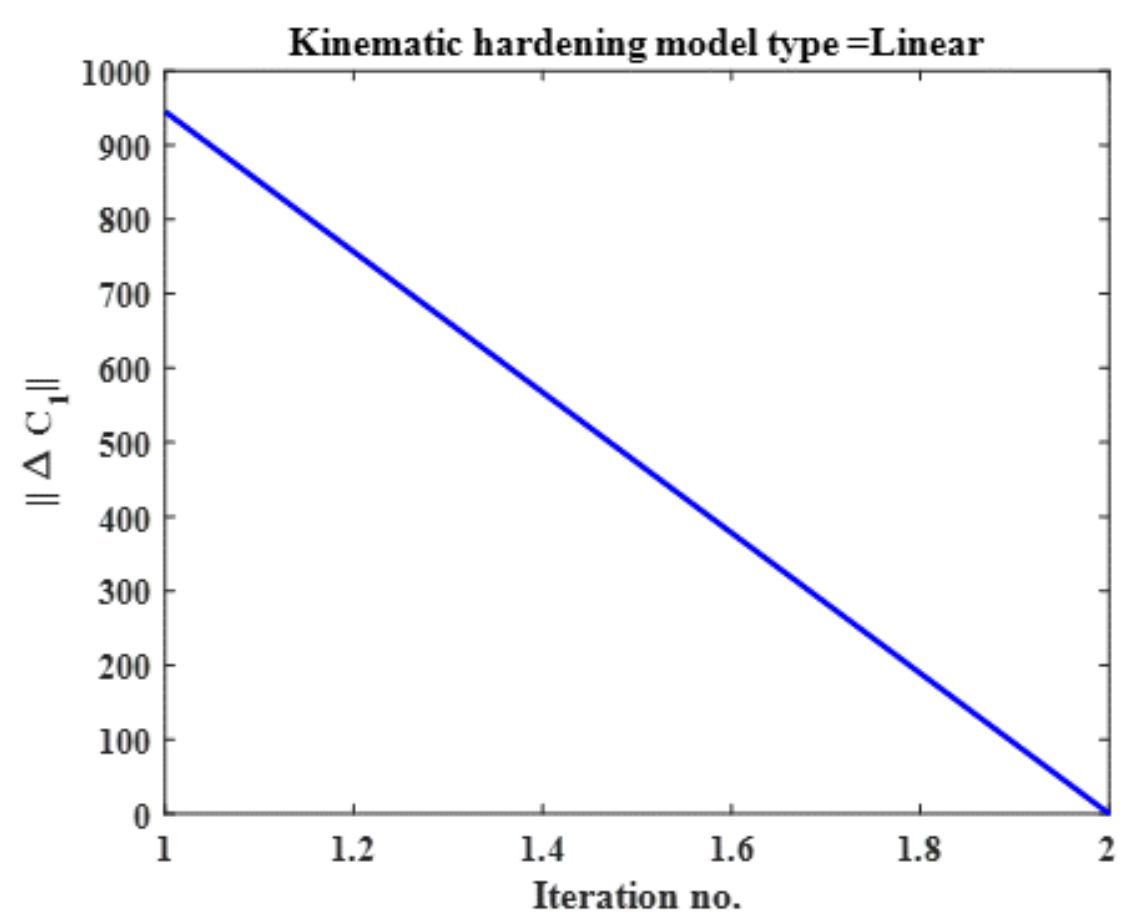

Figure 3A. $6 \mathrm{~L} 2$ norm (of incremental kinematic hardening constant $\mathrm{C} 1$ ) with respect to number of iteration during parameter estimation using gradient based optimization scheme and using ET-T04 stress-strain data (from elastic limit to $2 \%$ true total strain).

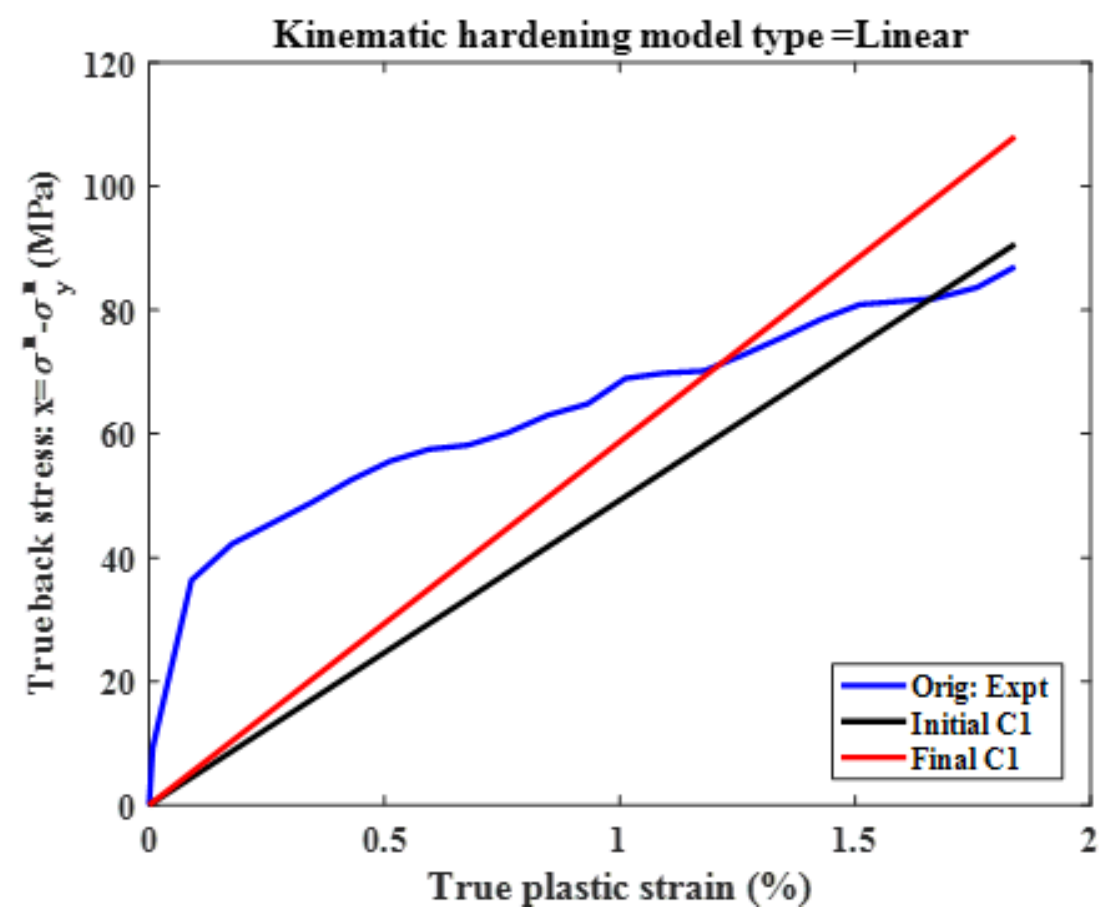

Figure 3A. 7 Comparison of predicted true back stress (using linear kinematic hardening parameter $\mathrm{C} 1$ and considering elastic limit as yield limit) with experimental true back stress for ET-T04 tensile test. 


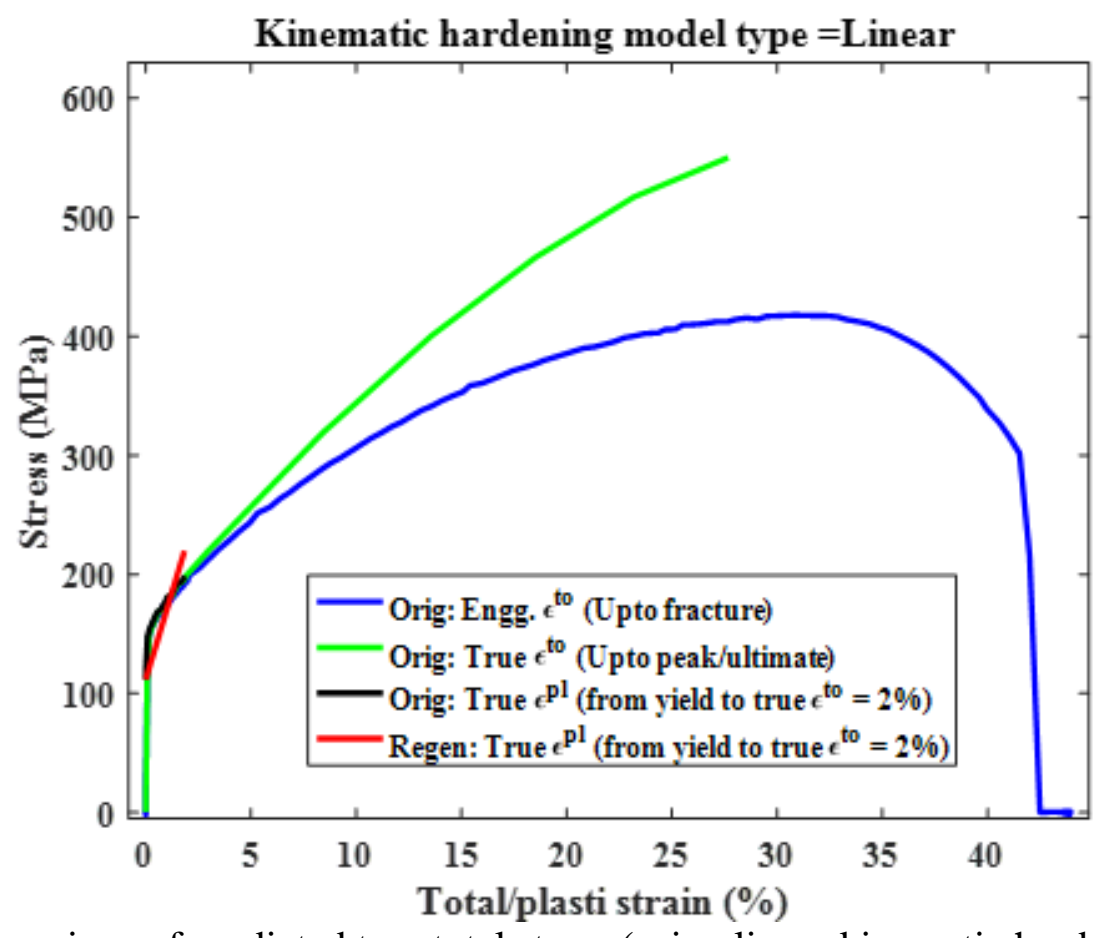

Figure 3A. 8 Comparison of predicted true total stress (using linear kinematic hardening parameter C1 and elastic limit as yield limit) with experimental true total stress for ET-T04 tensile test.

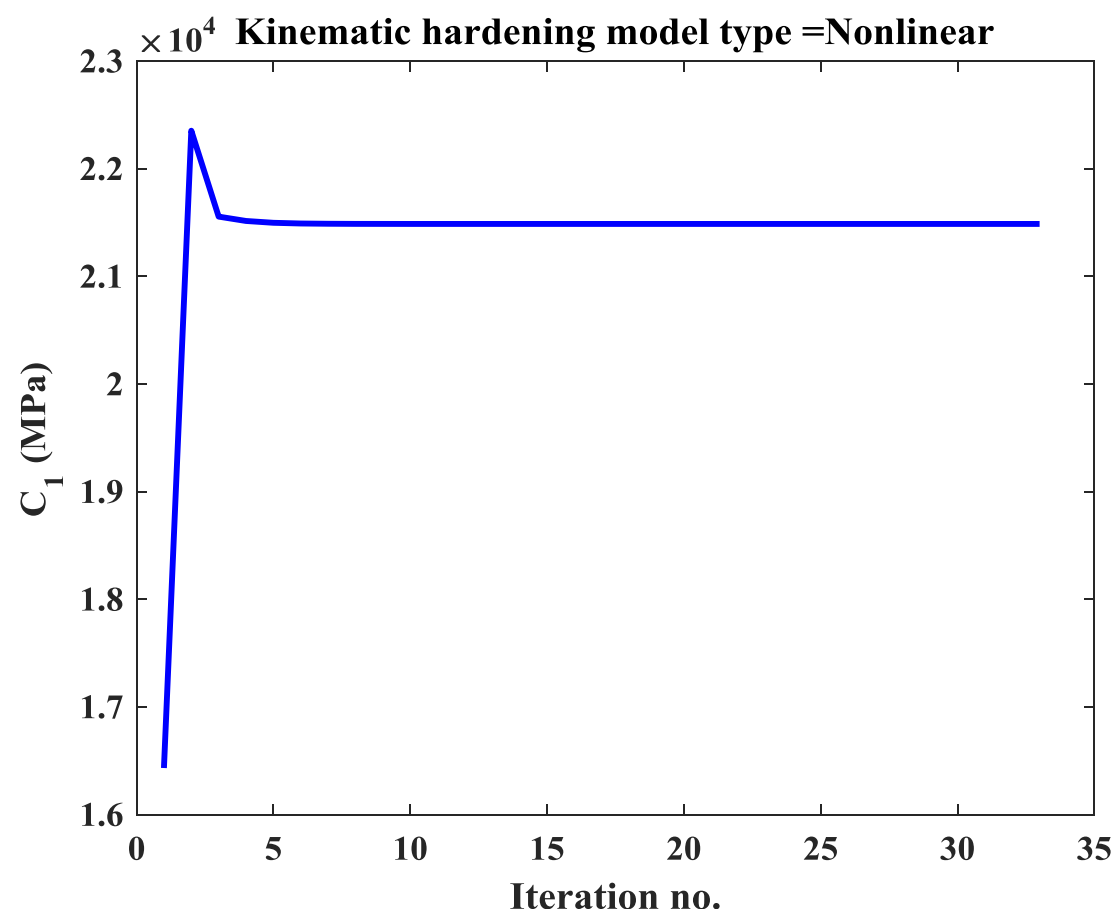

Figure 3A. 9 Nonlinear kinematic hardening constant C1 with respect to number of iteration during parameter estimation using gradient based optimization scheme and using ET-T04 stress-strain data (from elastic limit to $2 \%$ true total strain). 


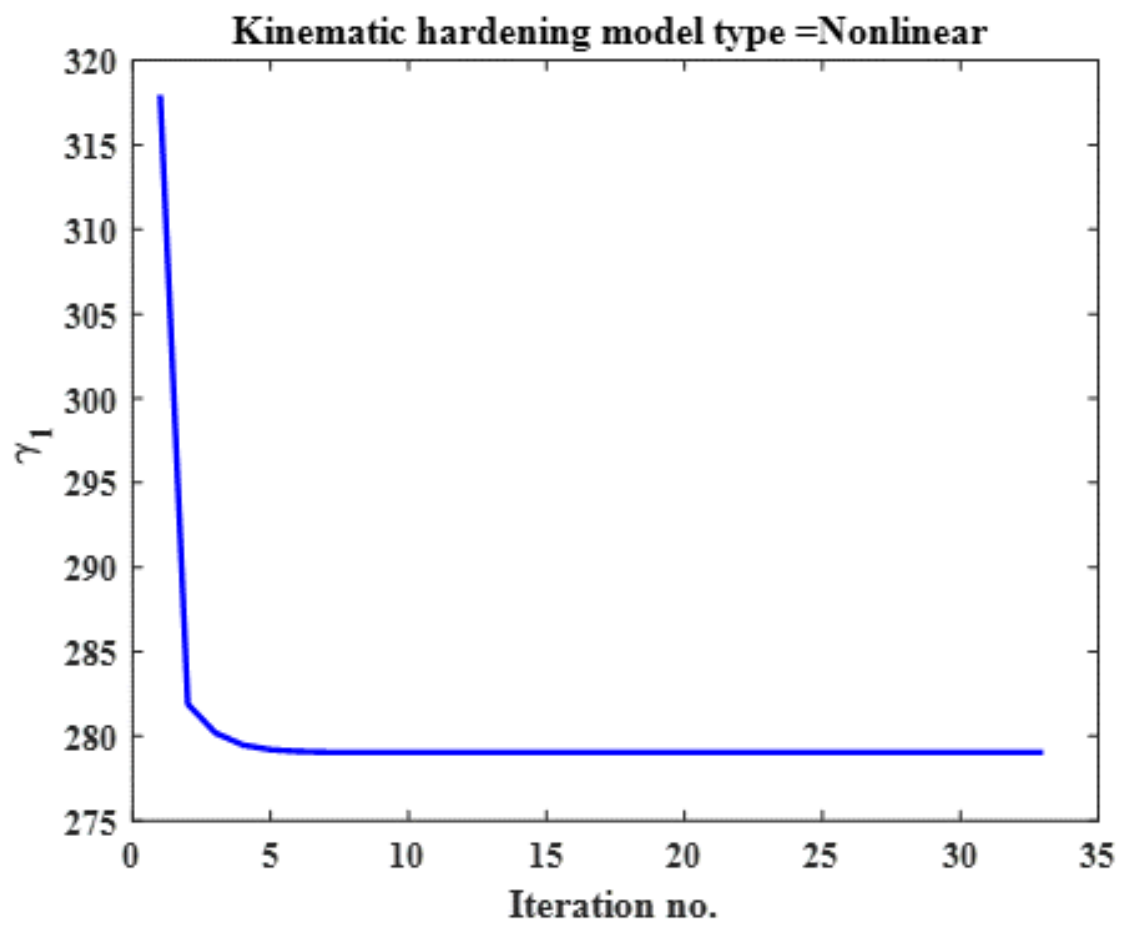

Figure 3A. 10 Nonlinear kinematic hardening constant $\gamma 1$ with respect to number of iteration during parameter estimation using gradient based optimization scheme and using ET-T04 stress-strain data (from elastic limit to $2 \%$ true total strain)

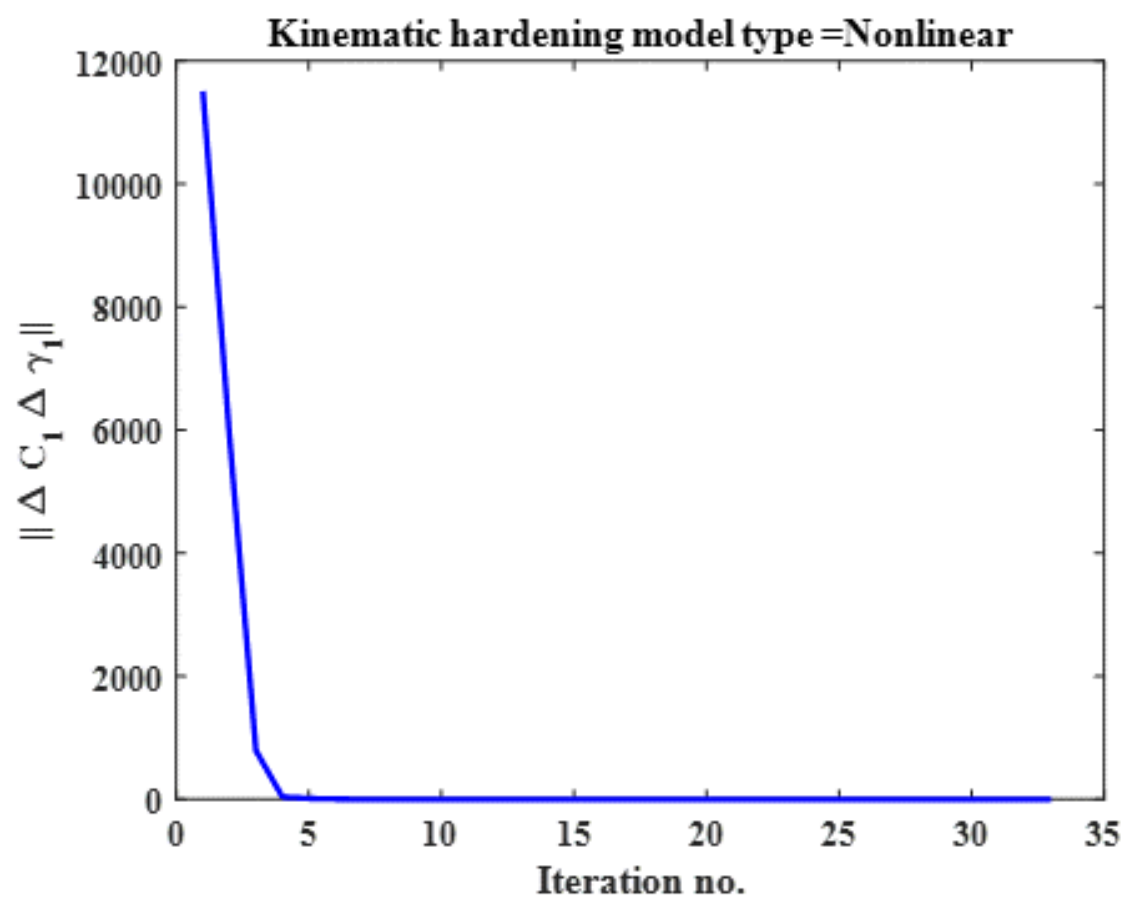

Figure 3A. 11 L2 norm (of incremental kinematic hardening constant $\mathrm{C} 1$ and $\gamma 1$ ) with respect to number of iteration during parameter estimation using gradient based optimization scheme and using ET-T04 stress-strain data (from elastic limit to $2 \%$ true total strain). 


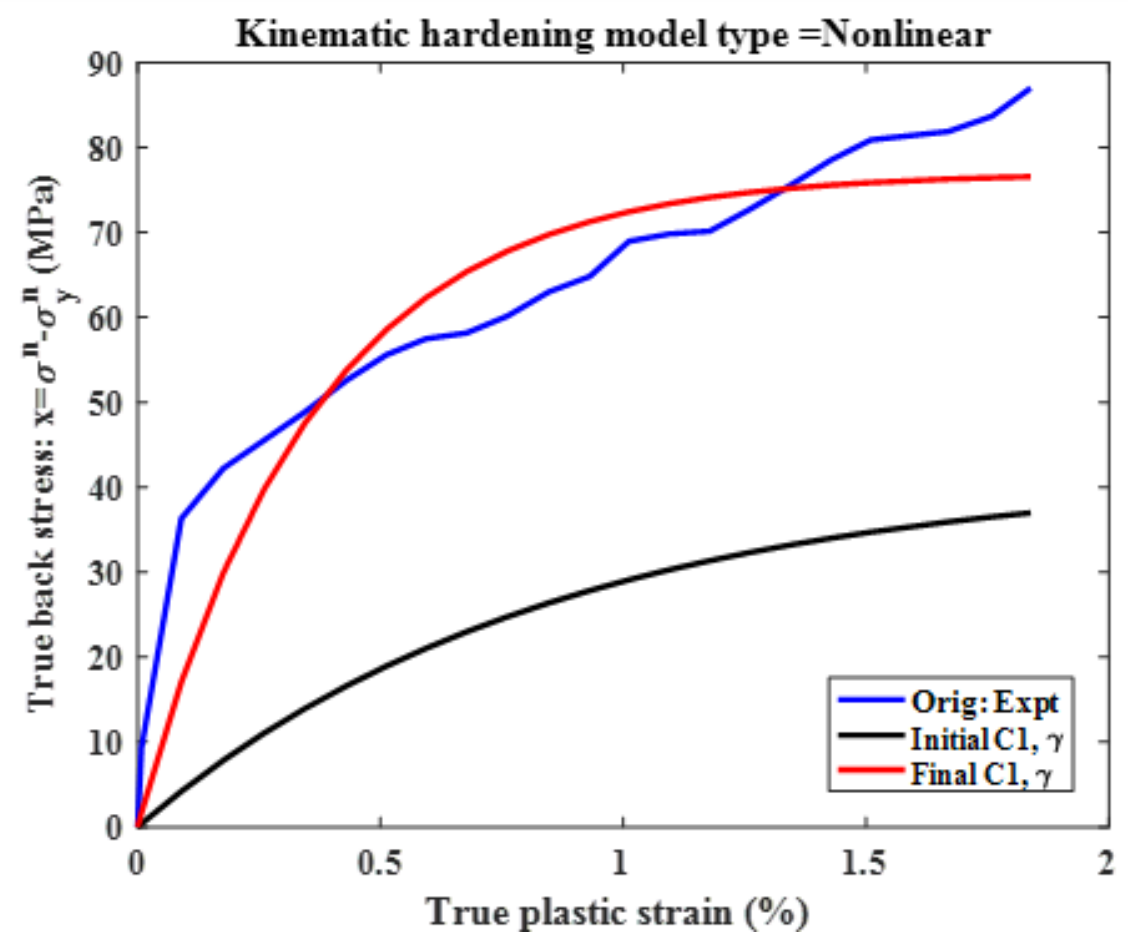

Figure 3A. 12 Comparison of predicted true back stress (using nonlinear kinematic hardening parameter

$\mathrm{C} 1$ and $\gamma 1$ and considering elastic limit as yield limit) with experimental true back stress for ET-T04 tensile test.

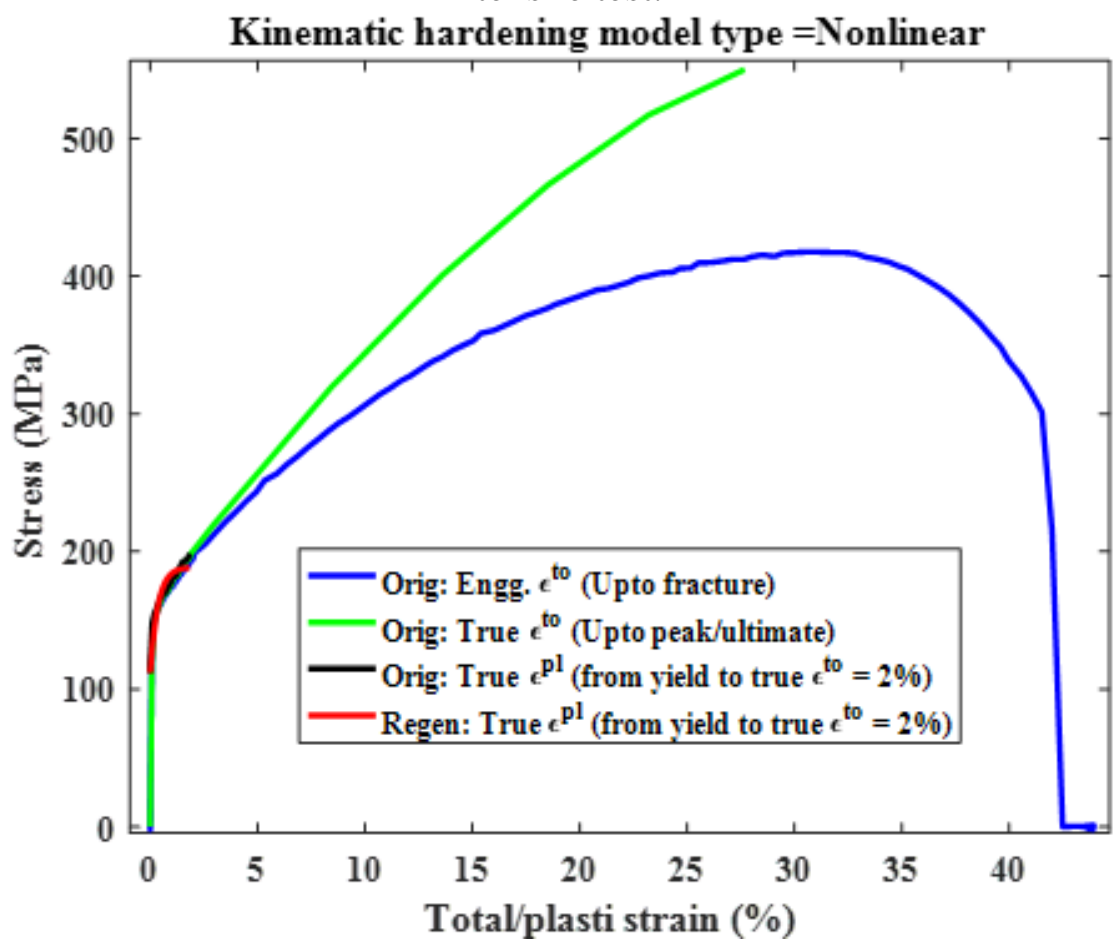

Figure 3A. 13 Comparison of predicted true total stress (using nonlinear kinematic hardening parameter $\mathrm{C} 1$ and $\gamma 1$ and considering elastic limit as yield limit) with experimental true total stress for ET-T04 tensile test. 


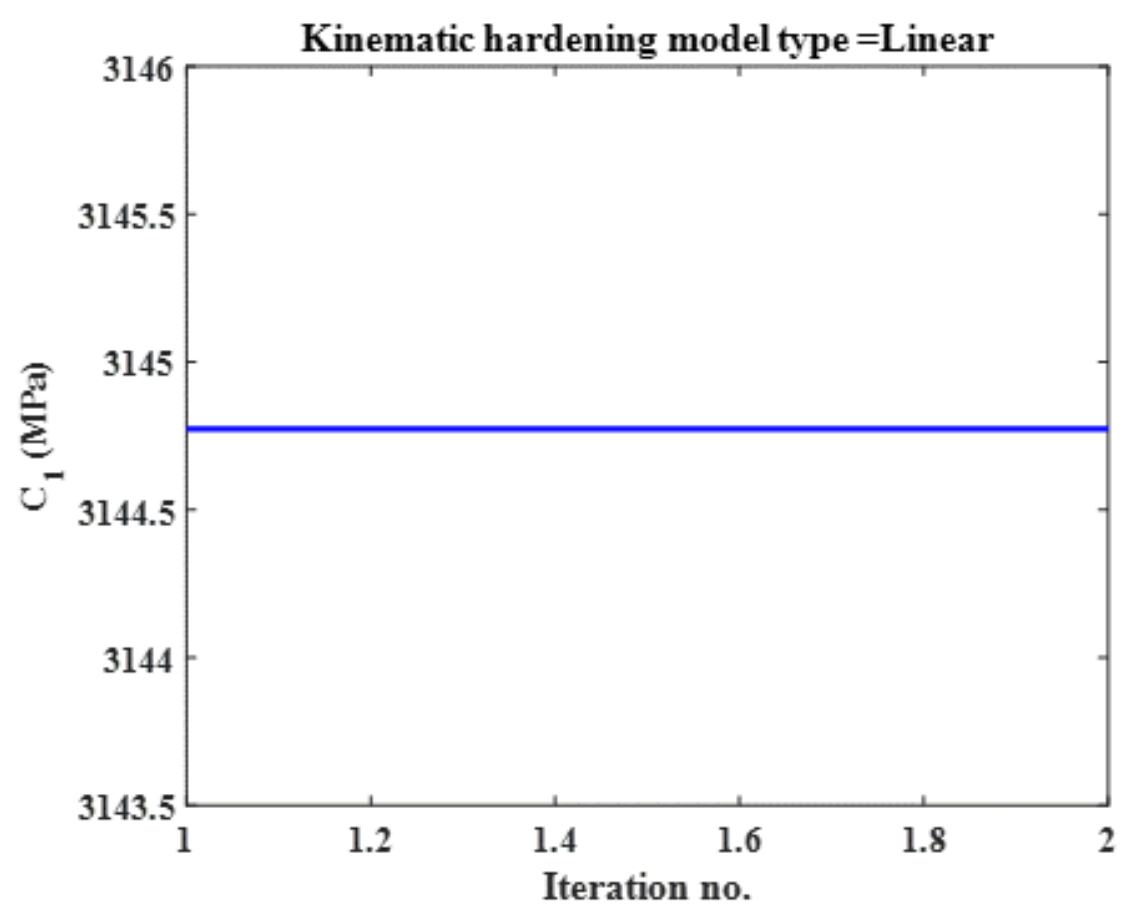

Figure 3A. 14 Linear kinematic hardening parameter $\mathrm{C} 1$ with respect to number of iteration during parameter estimation using gradient based optimization scheme and using ET-T04 stress-strain data (from $0.05 \%$ offset strain yield limit to $2 \%$ true total strain).

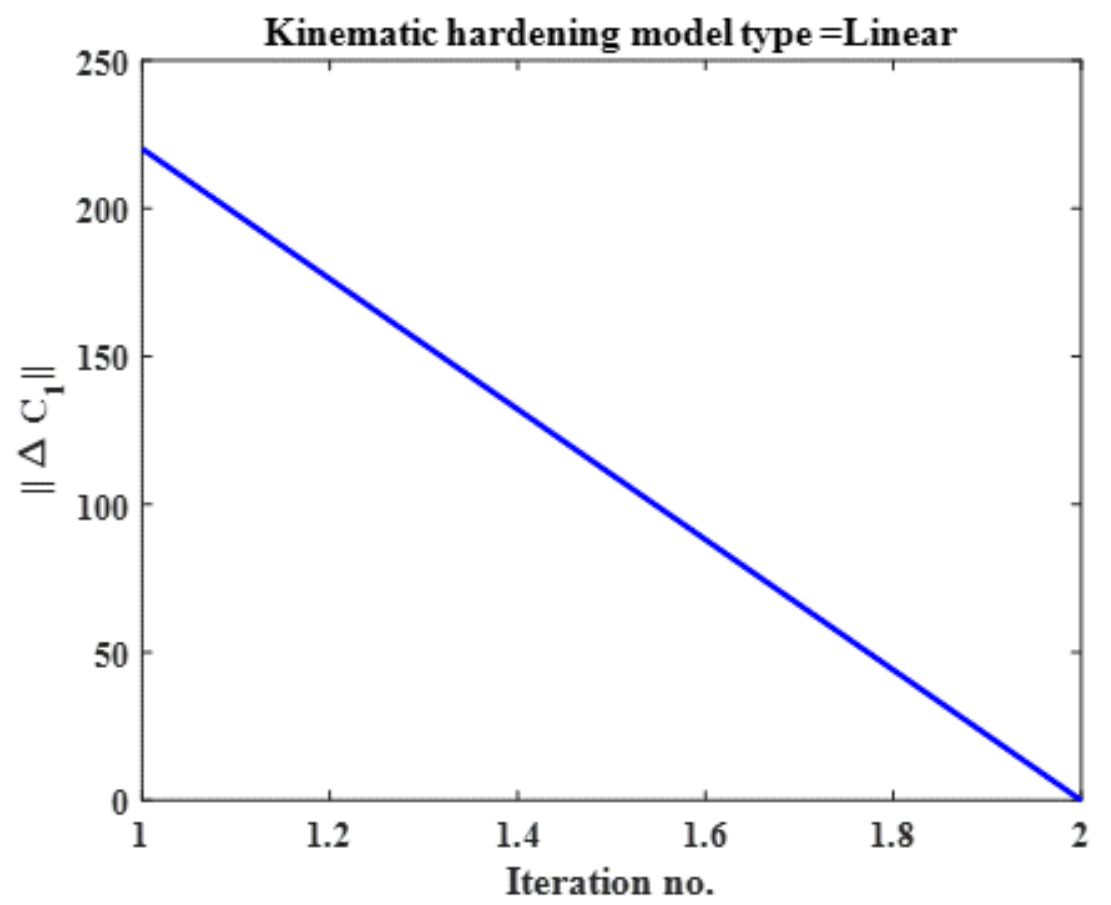

Figure 3A. $15 \mathrm{~L} 2$ norm (of incremental kinematic hardening constant $\mathrm{C} 1$ ) with respect to number of iteration during parameter estimation using gradient based optimization scheme and using ET-T04 stress-strain data (from $0.05 \%$ offset strain yield limit to $2 \%$ true total strain). 


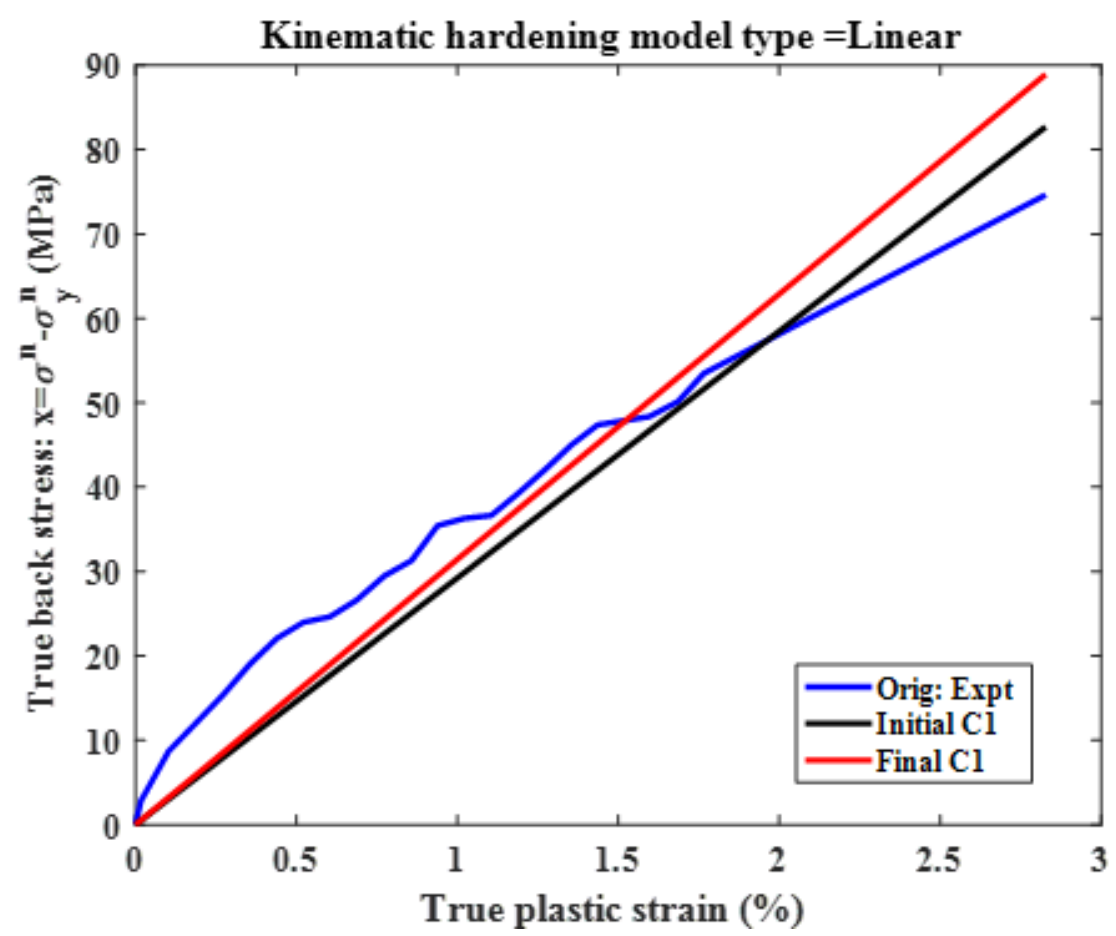

Figure 3A. 16 Comparison of predicted true back stress (using linear kinematic hardening parameter $\mathrm{C} 1$ and considering $0.05 \%$ offset strain as yield limit) with experimental true back stress for ET-T04 tensile test.

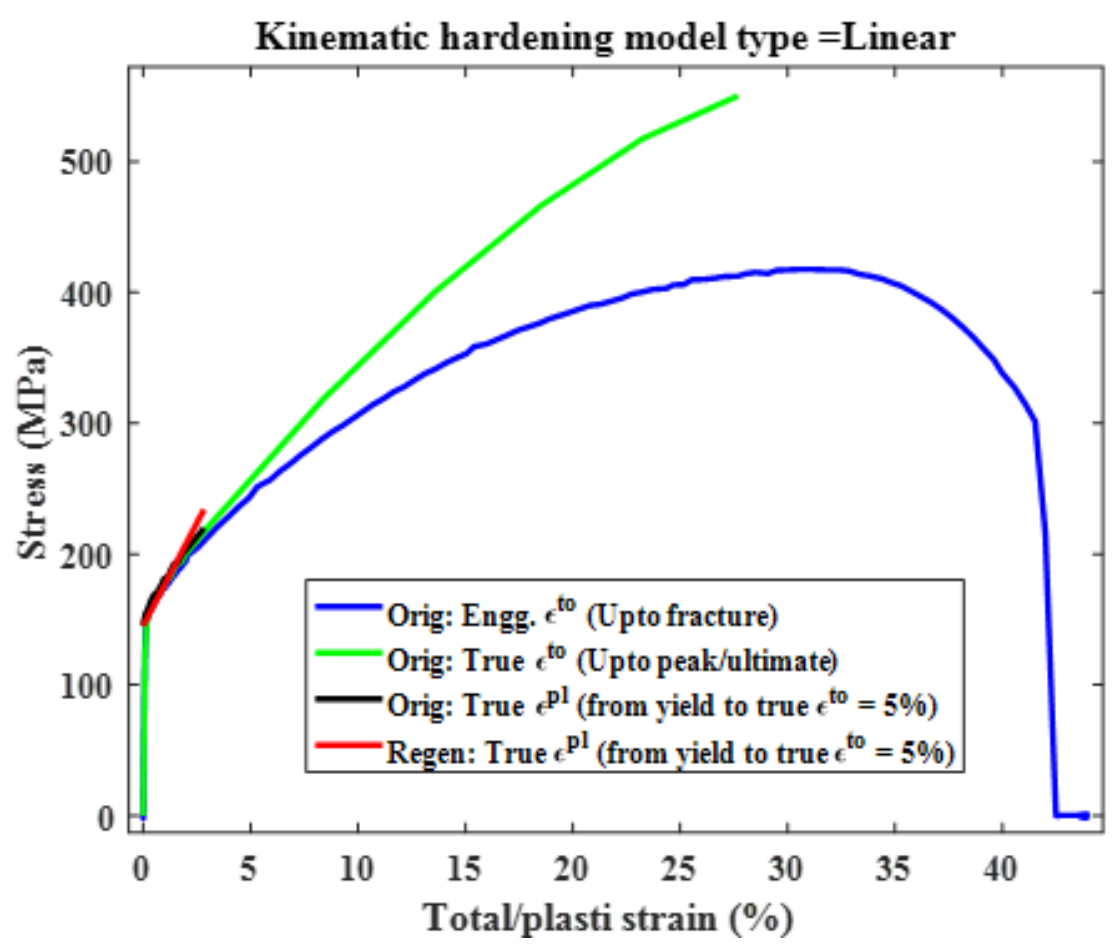

Figure 3A. 17 Comparison of predicted true total stress (using linear kinematic hardening parameter $\mathrm{C} 1$ and $0.05 \%$ offset strain as yield limit) with experimental true total stress for ET-T04 tensile test. 


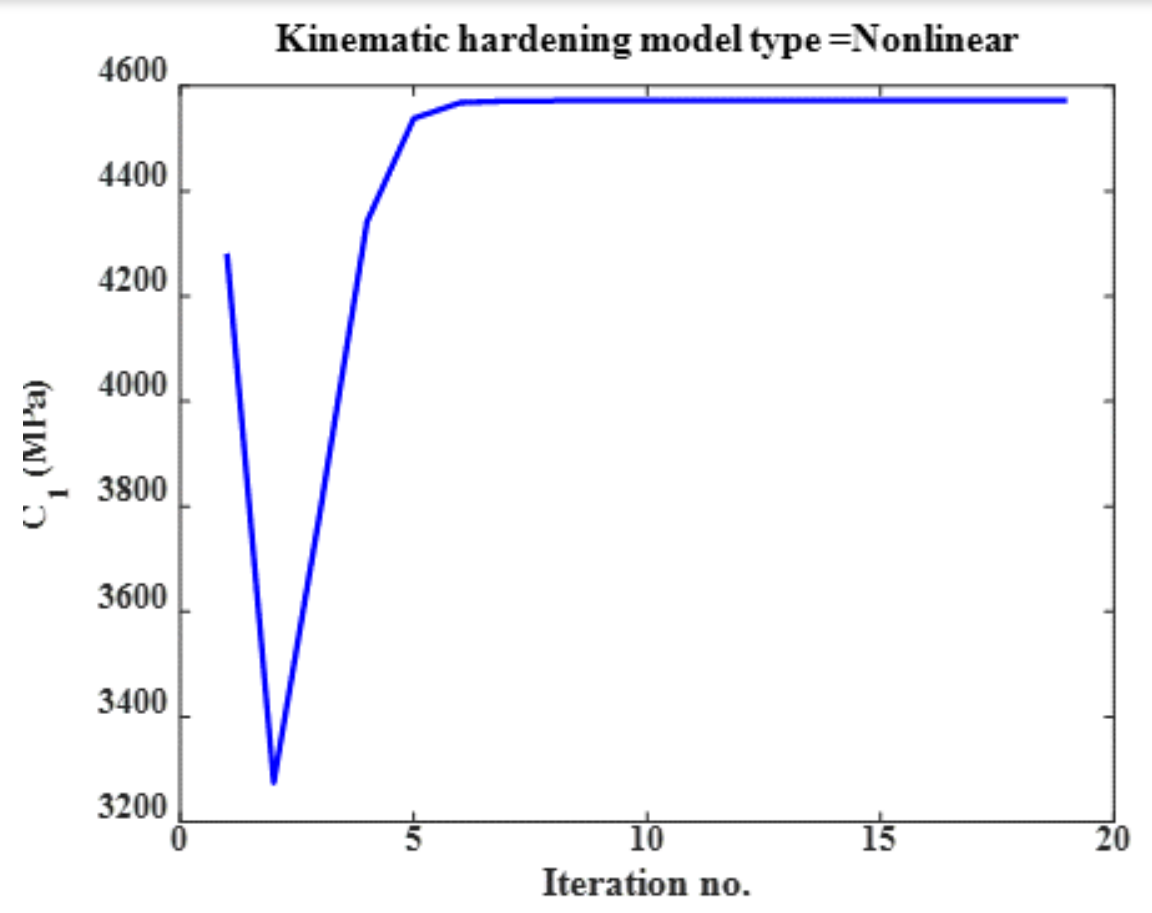

Figure 3A. 18 Nonlinear kinematic hardening constant $\mathrm{C} 1$ with respect to number of iteration during parameter estimation using gradient based optimization scheme and using ET-T04 stress-strain data (from $0.05 \%$ offset yield strain limit to $2 \%$ true total strain)

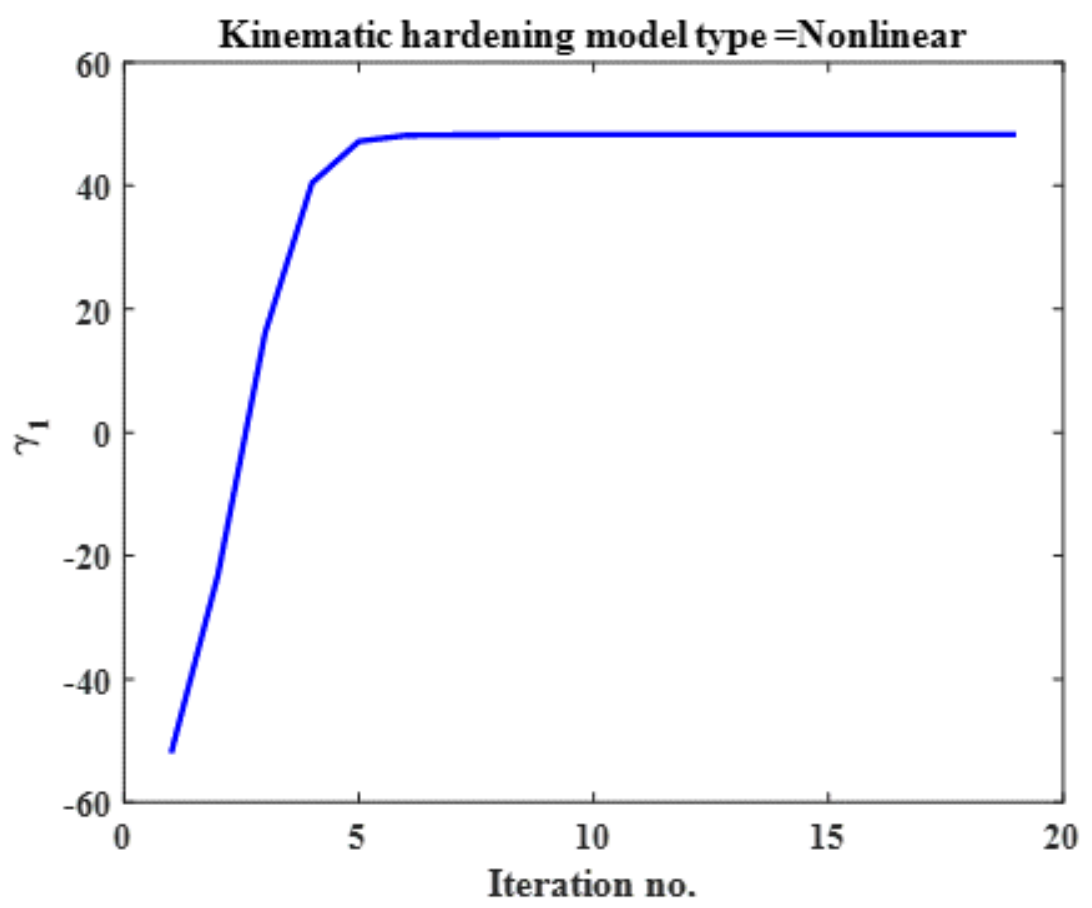

Figure 3A. 19 Nonlinear kinematic hardening constant $\gamma 1$ with respect to number of iteration during parameter estimation using gradient based optimization scheme and using ET-T04 stress-strain data (from $0.05 \%$ offset yield strain limit to $2 \%$ true total strain) 


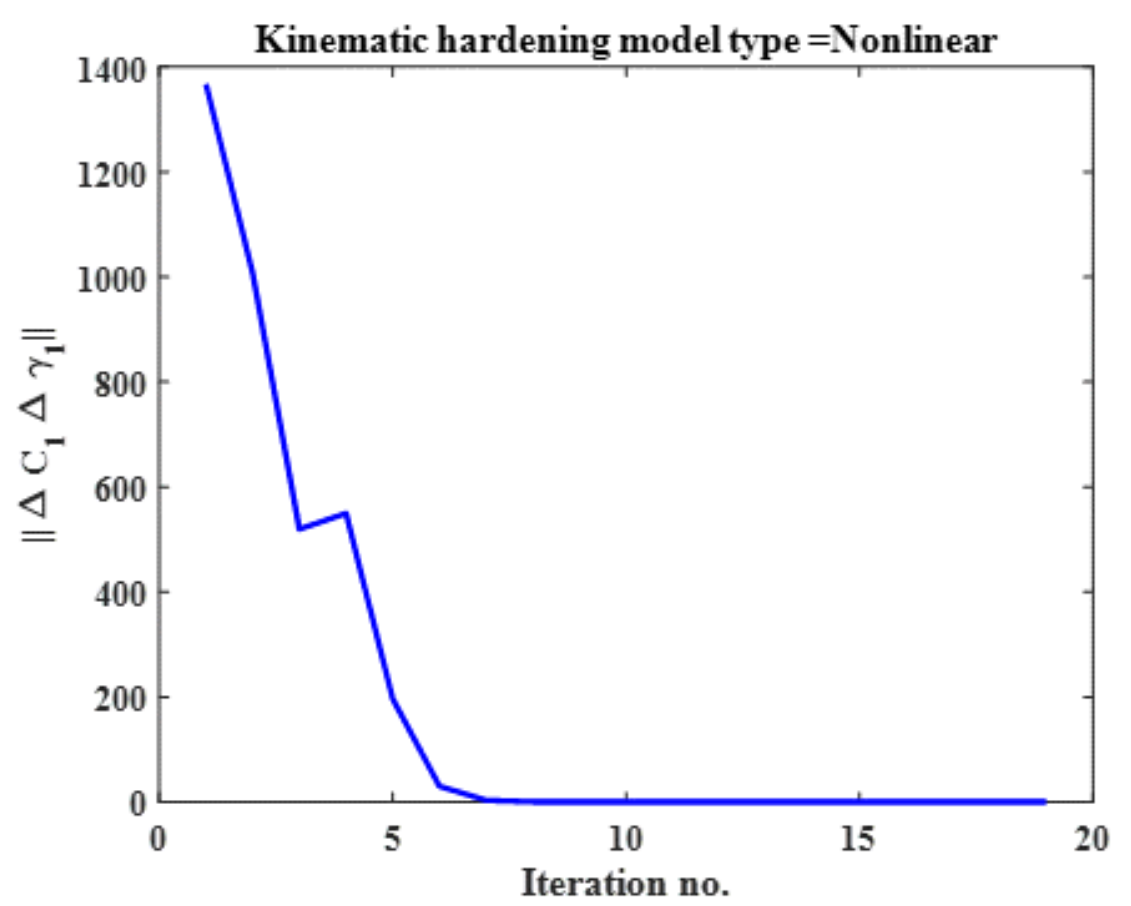

Figure 3A. 20 L2 norm (of incremental kinematic hardening constant $\mathrm{C} 1$ and $\gamma 1$ ) with respect to number of iteration during parameter estimation using gradient based optimization scheme and using ET-T04 stress-strain data (from $0.05 \%$ offset yield strain limit to $2 \%$ true total strain).

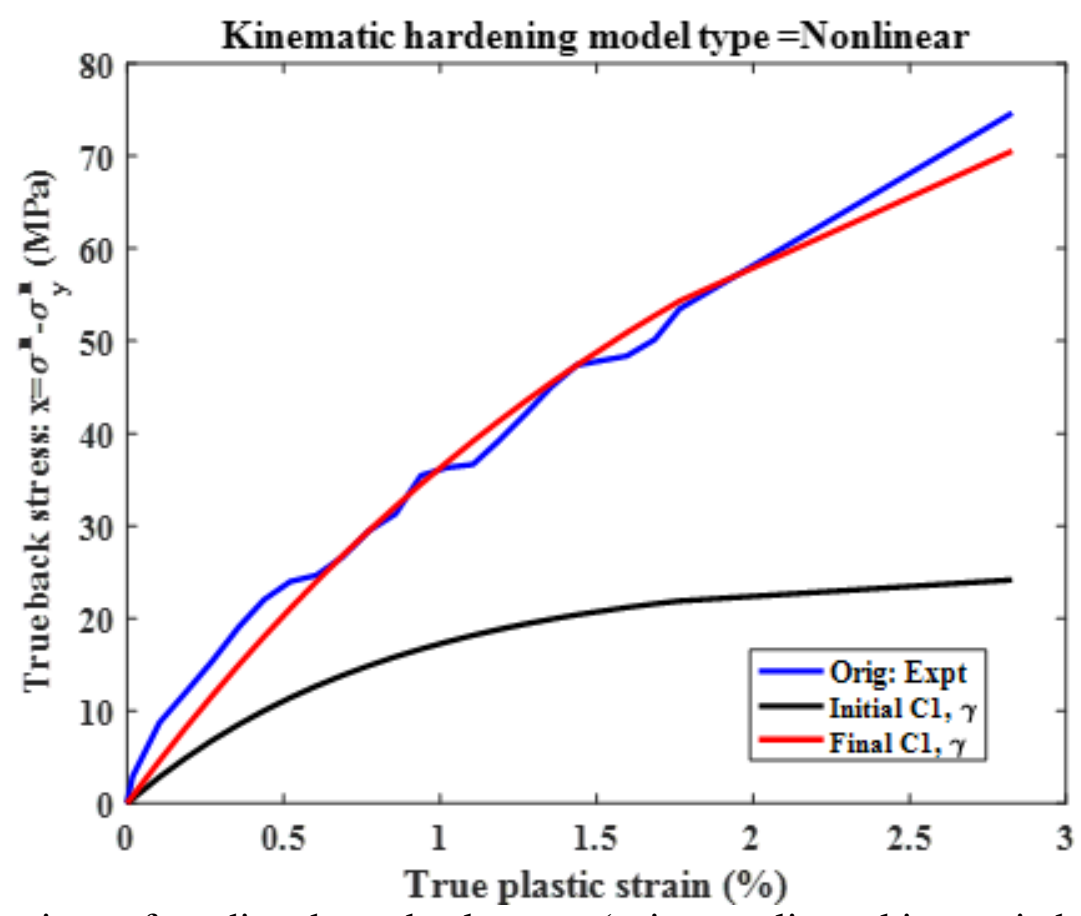

Figure 3A. 21 Comparison of predicted true back stress (using nonlinear kinematic hardening parameter $\mathrm{C} 1$ and $\gamma 1$ and considering $0.05 \%$ offset yield strain as yield limit) with experimental true back stress for ET-T04 tensile test. 


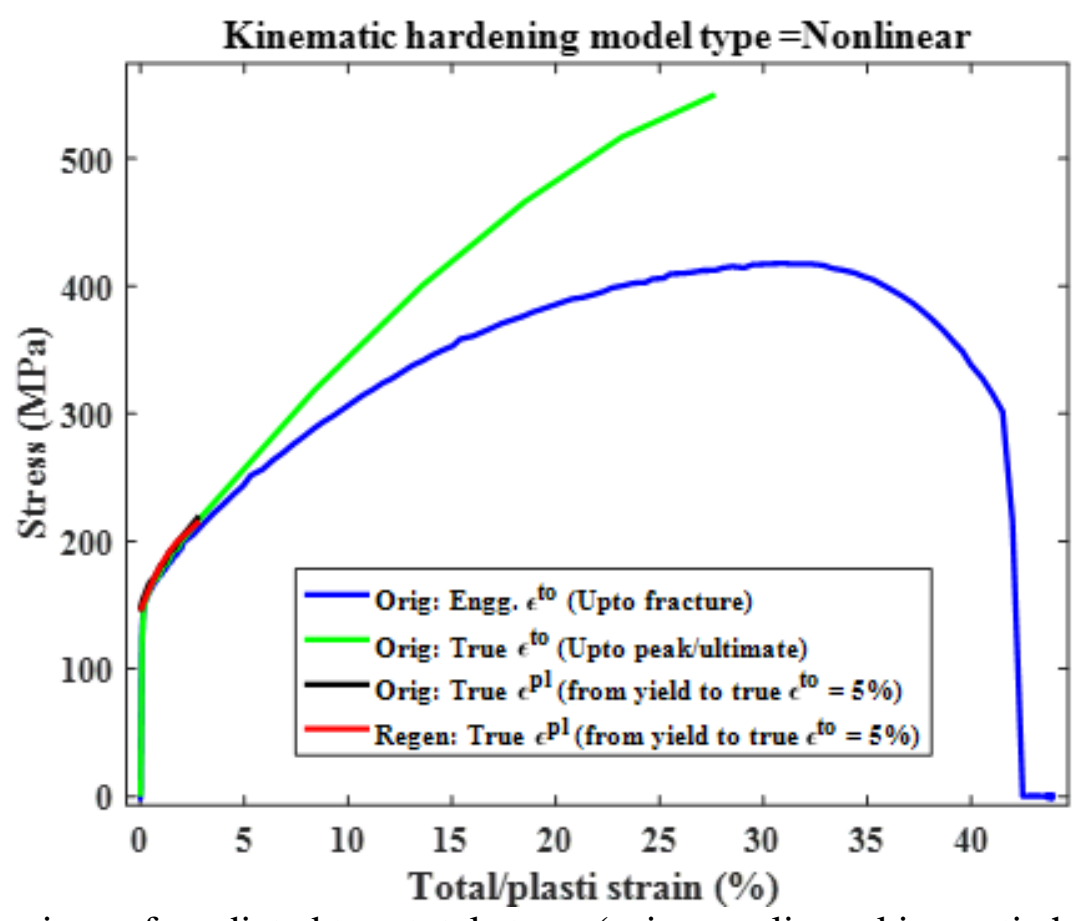

Figure 3A. 22 Comparison of predicted true total stress (using nonlinear kinematic hardening parameter $\mathrm{C} 1$ and $\gamma 1$ and considering $0.05 \%$ offset yield strain as yield limit) with experimental true total stress for ET-T04 tensile test.

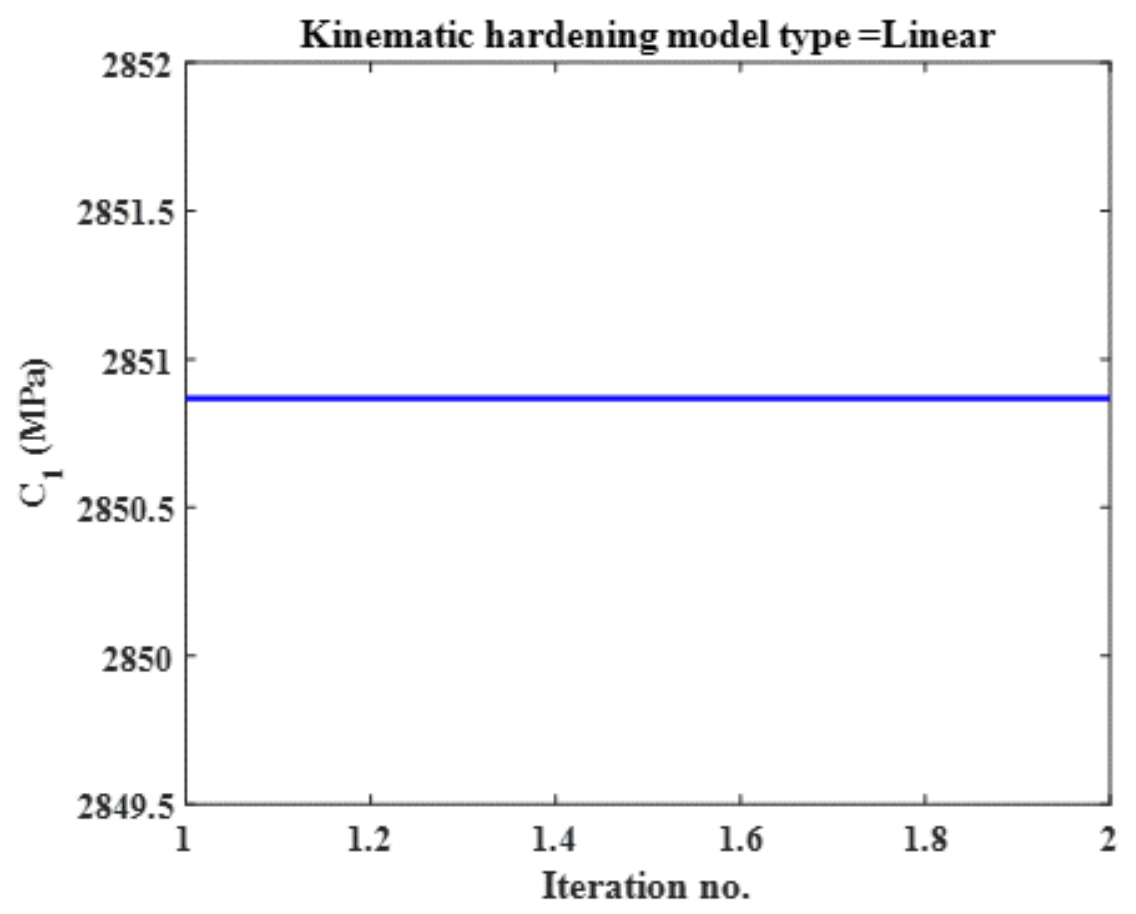

Figure 3A. 23 Linear kinematic hardening parameter $\mathrm{C} 1$ with respect to number of iteration during parameter estimation using gradient based optimization scheme and using ET-T04 stress-strain data (from $0.1 \%$ offset strain yield limit to $2 \%$ true total strain). 


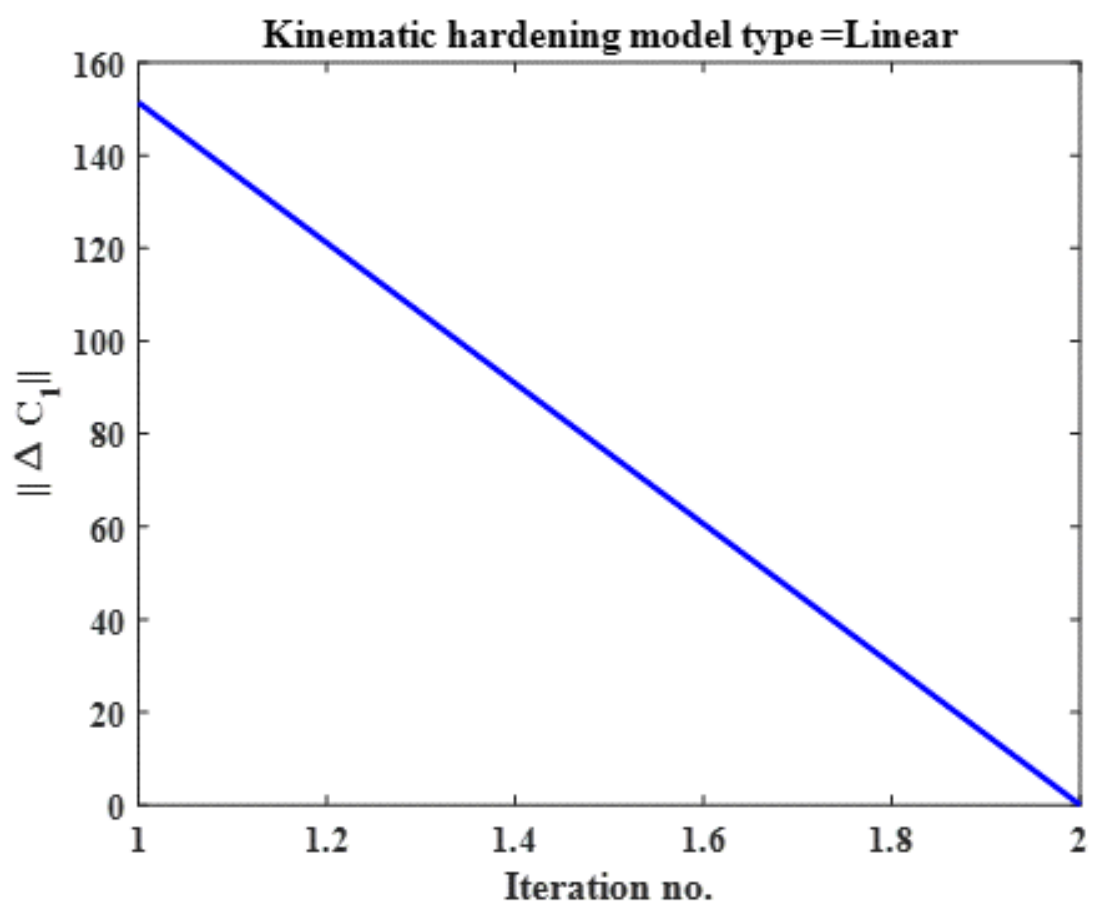

Figure 3A. $24 \mathrm{~L} 2$ norm (of incremental kinematic hardening constant C1) with respect to number of iteration during parameter estimation using gradient based optimization scheme and using ET-T04 stress-strain data (from $0.1 \%$ offset strain yield limit to $2 \%$ true total strain).

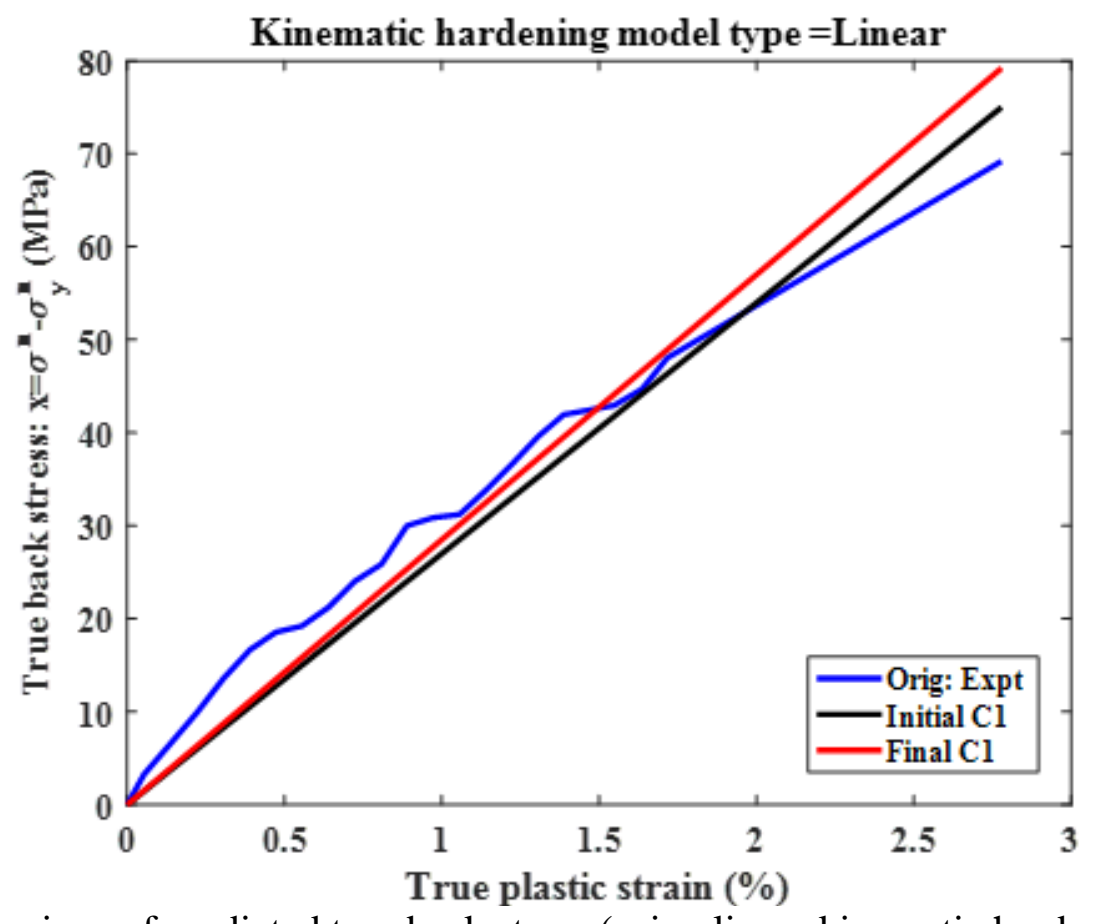

Figure 3A. 25 Comparison of predicted true back stress (using linear kinematic hardening parameter $\mathrm{C} 1$ and considering $0.1 \%$ offset strain as yield limit) with experimental true back stress for ET-T04 tensile test. 


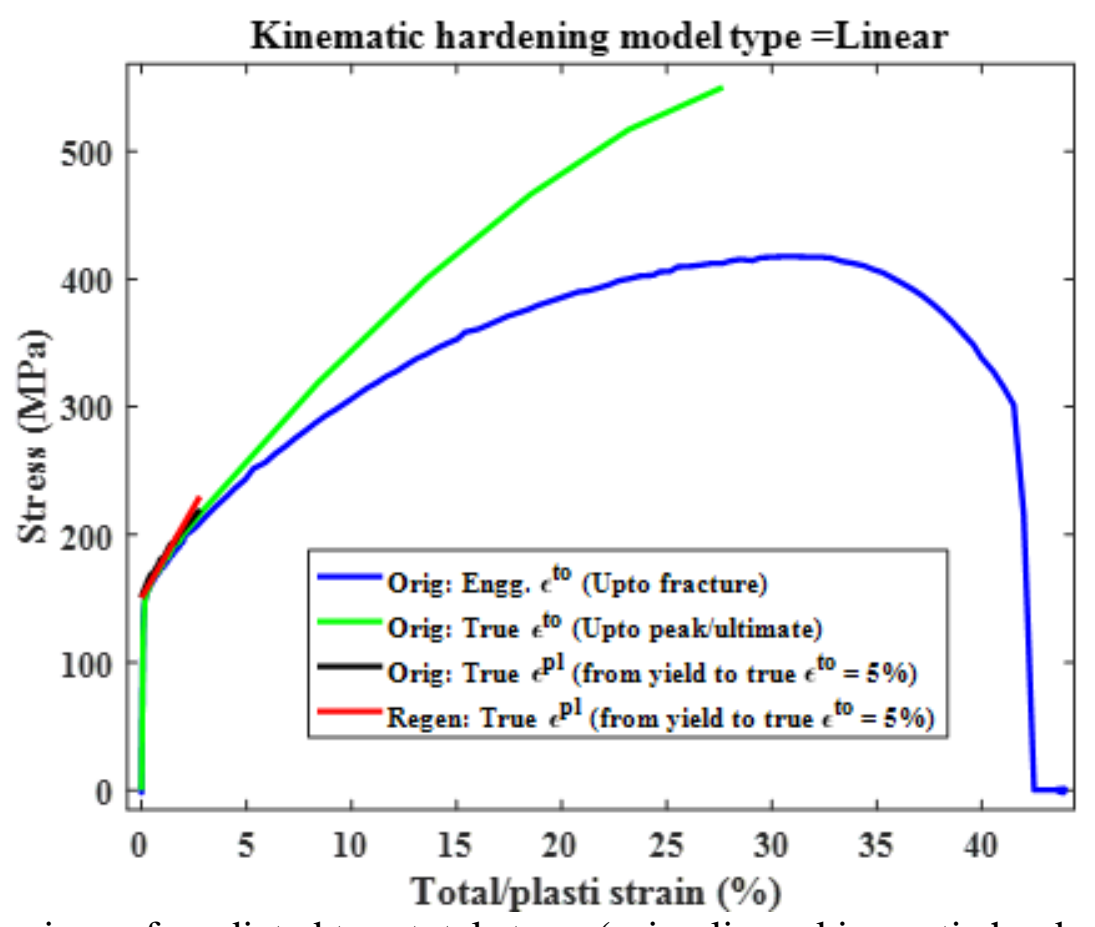

Figure 3A. 26 Comparison of predicted true total stress (using linear kinematic hardening parameter $\mathrm{C} 1$ and $0.1 \%$ offset strain as yield limit) with experimental true total stress for ET-T04 tensile test.

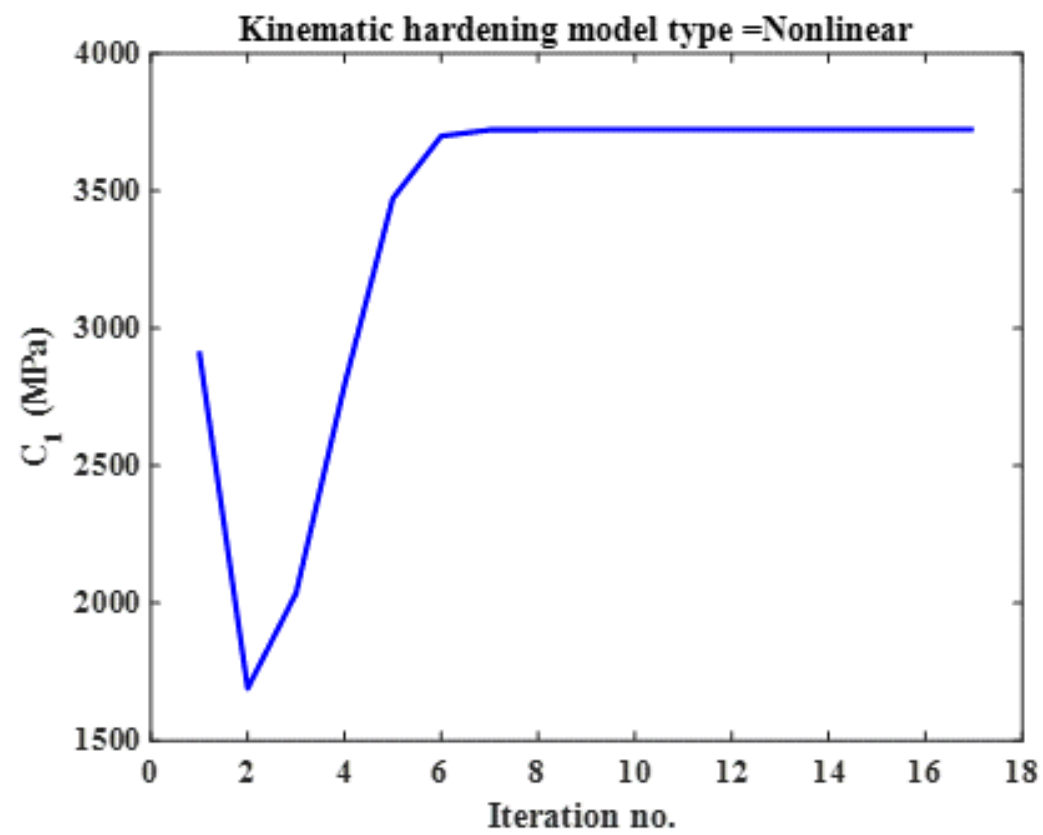

Figure 3A. 27 Nonlinear kinematic hardening constant C1 with respect to number of iteration during parameter estimation using gradient based optimization scheme and using ET-T04 stress-strain data (from $0.1 \%$ offset yield strain limit to $2 \%$ true total strain) 


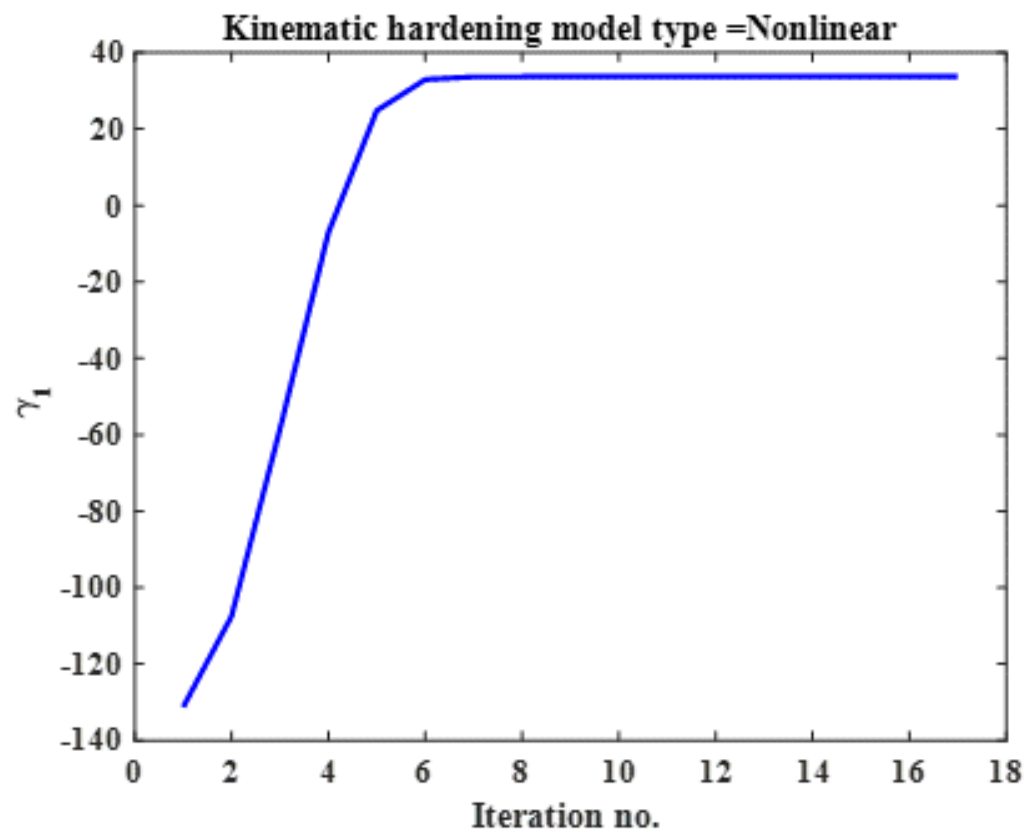

Figure 3A. 28 Nonlinear kinematic hardening constant $\gamma 1$ with respect to number of iteration during parameter estimation using gradient based optimization scheme and using ET-T04 stress-strain data (from $0.1 \%$ offset yield strain limit to $2 \%$ true total strain)

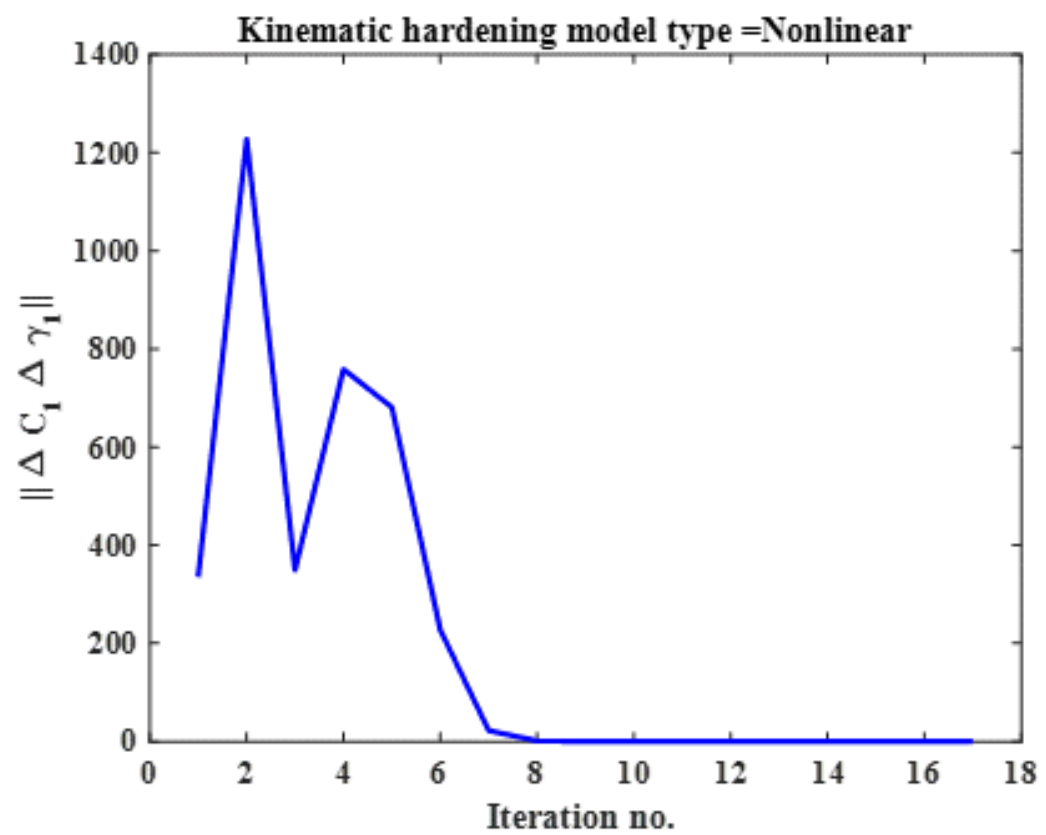

Figure 3A. 29 L2 norm (of incremental kinematic hardening constant $\mathrm{C} 1$ and $\gamma 1$ ) with respect to number of iteration during parameter estimation using gradient based optimization scheme and using ET-T04 stress-strain data (from $0.1 \%$ offset yield strain limit to $2 \%$ true total strain). 


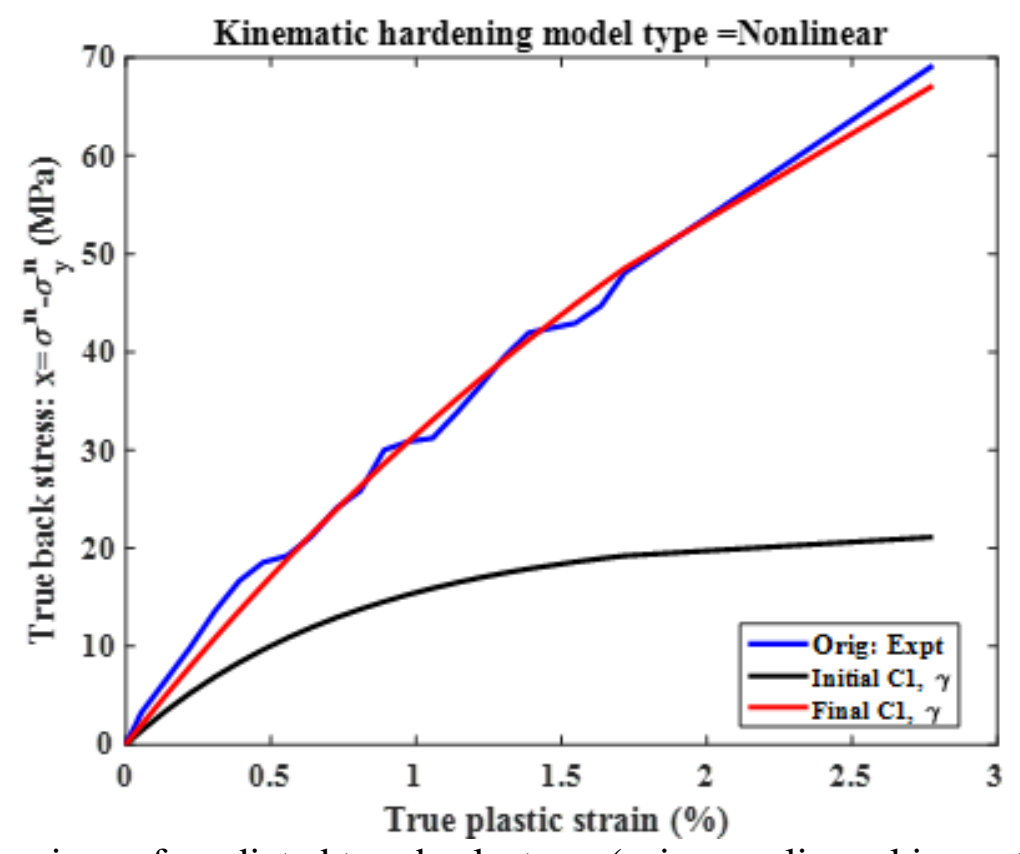

Figure 3A. 30 Comparison of predicted true back stress (using nonlinear kinematic hardening parameter $\mathrm{C} 1$ and $\gamma 1$ and considering $0.1 \%$ offset yield strain as yield limit) with experimental true back stress for ET-T04 tensile test.

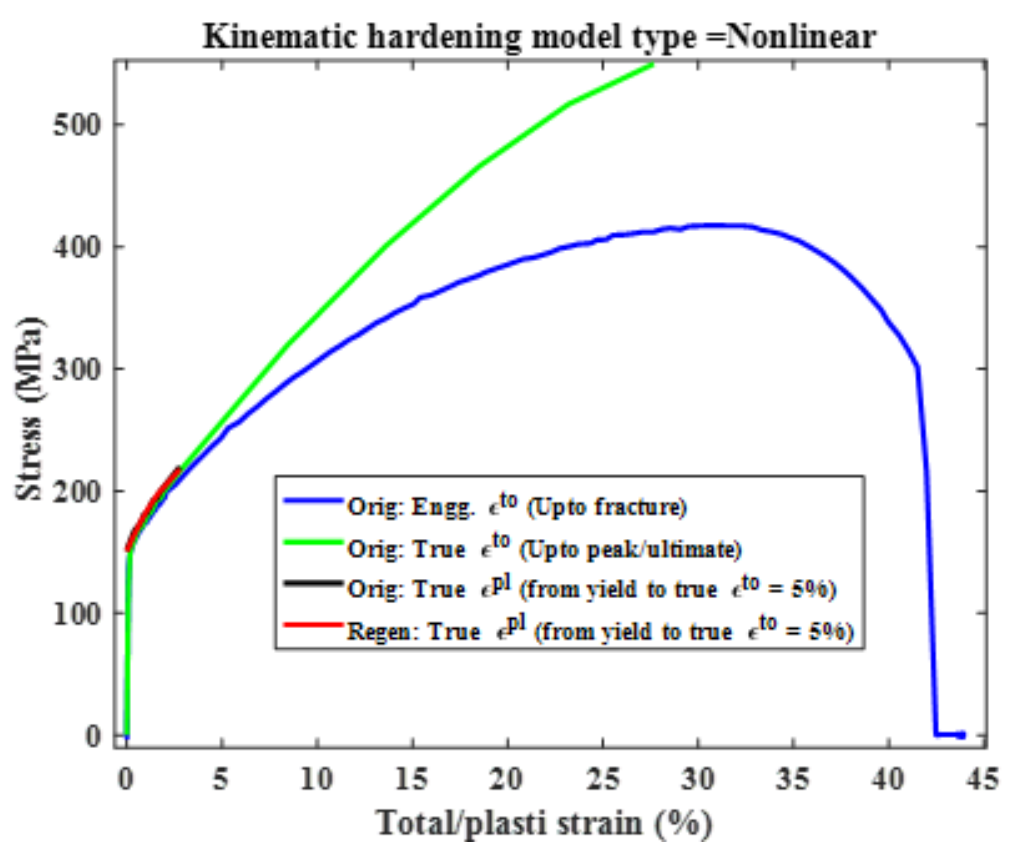

Figure 3A. 31 Comparison of predicted true total stress (using nonlinear kinematic hardening parameter $\mathrm{C} 1$ and $\gamma 1$ and considering $0.1 \%$ offset yield strain as yield limit) with experimental true total stress for ET-T04 tensile test. 


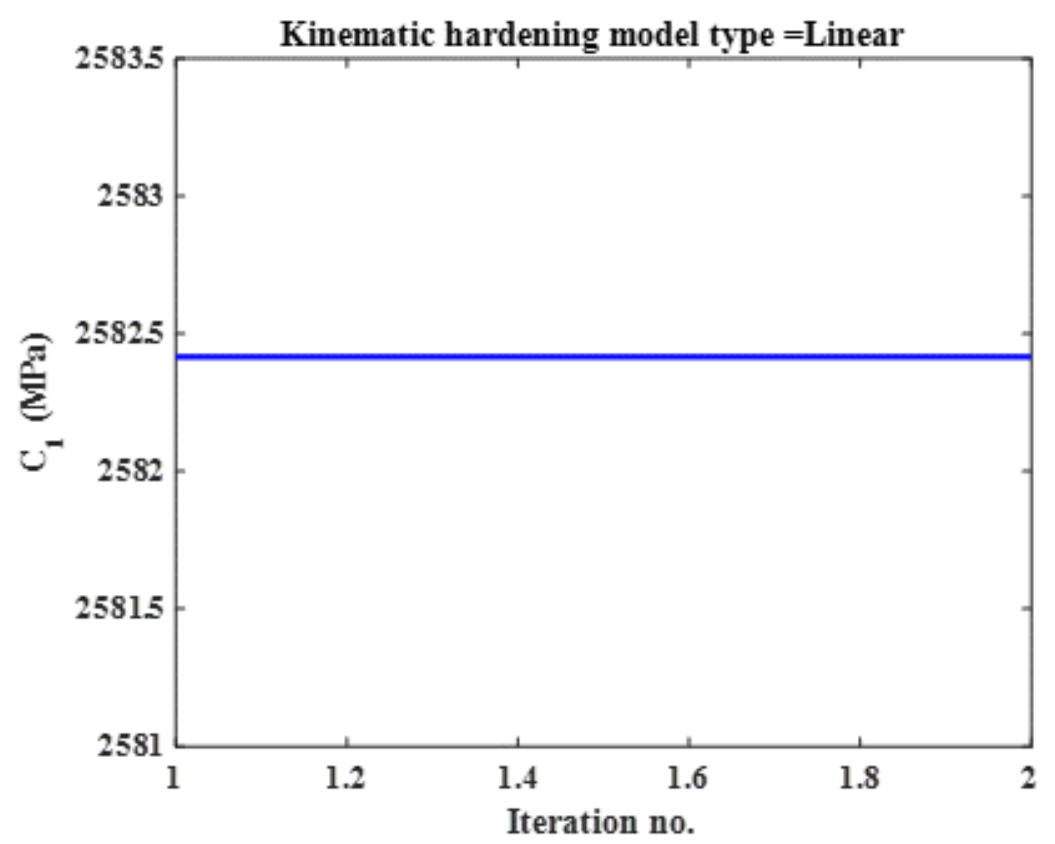

Figure 3A. 32 Linear kinematic hardening parameter $\mathrm{C} 1$ with respect to number of iteration during parameter estimation using gradient based optimization scheme and using ET-T04 stress-strain data (from $0.2 \%$ offset strain yield limit to $2 \%$ true total strain).

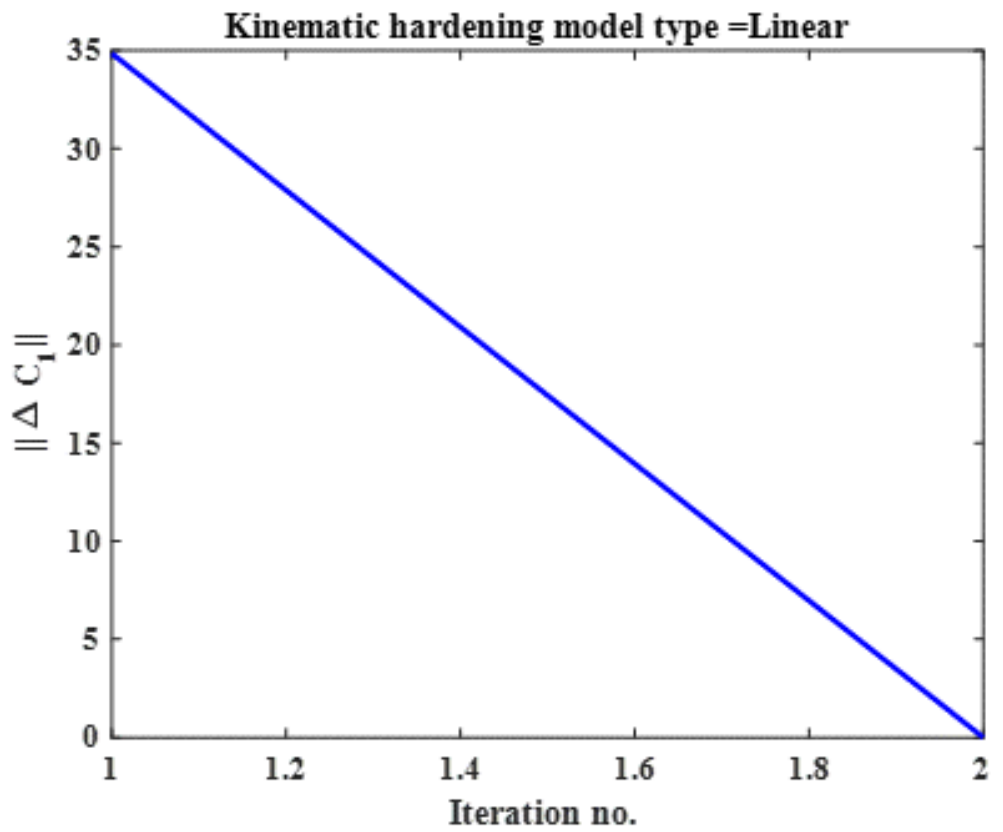

Figure 3A. 33 L2 norm (of incremental kinematic hardening constant C1) with respect to number of iteration during parameter estimation using gradient based optimization scheme and using ET-T04 stress-strain data (from $0.2 \%$ offset strain yield limit to $2 \%$ true total strain). 


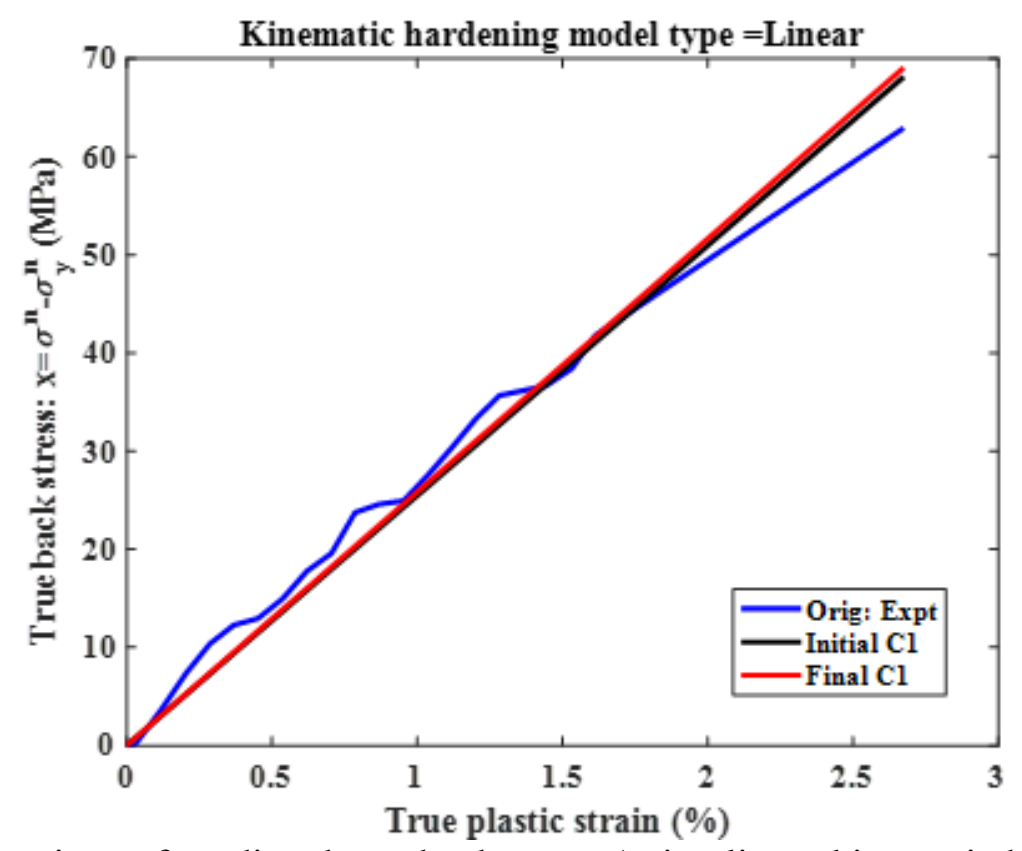

Figure 3A. 34 Comparison of predicted true back stress (using linear kinematic hardening parameter $\mathrm{C} 1$ and considering $0.2 \%$ offset strain as yield limit) with experimental true back stress for ET-T04 tensile test.

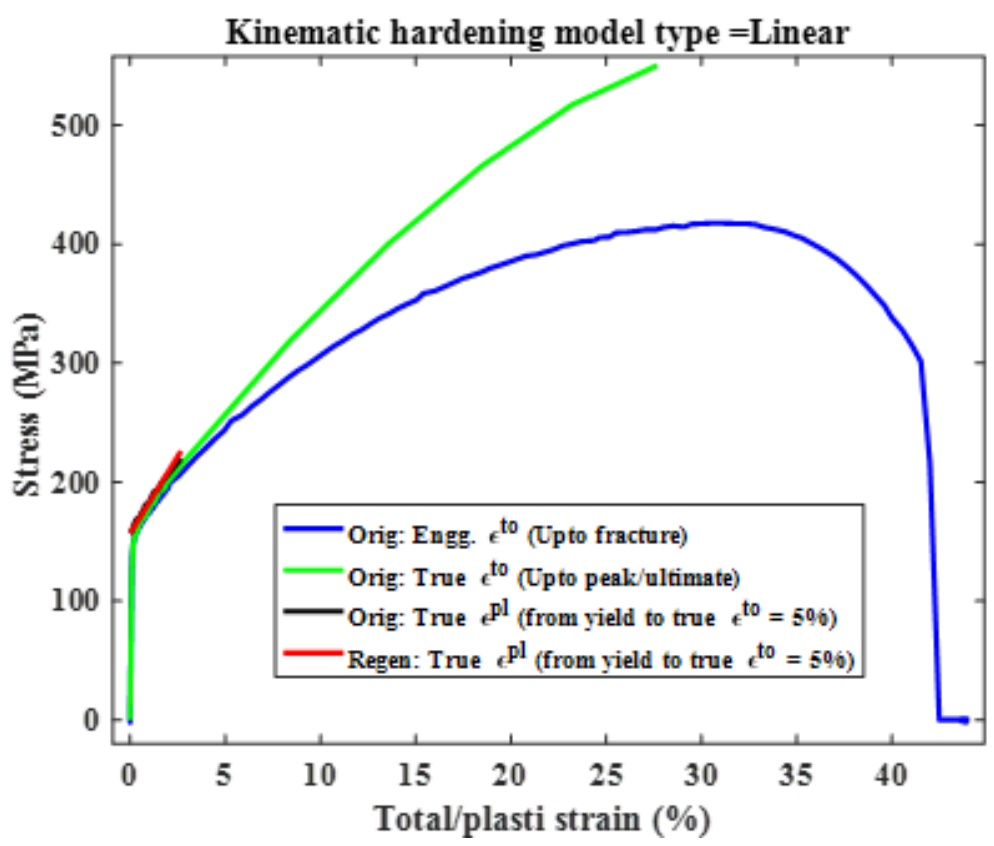

Figure 3A. 35 Comparison of predicted true total stress (using linear kinematic hardening parameter $\mathrm{C} 1$ and $0.2 \%$ offset strain as yield limit) with experimental true total stress for ET-T04 tensile test. 


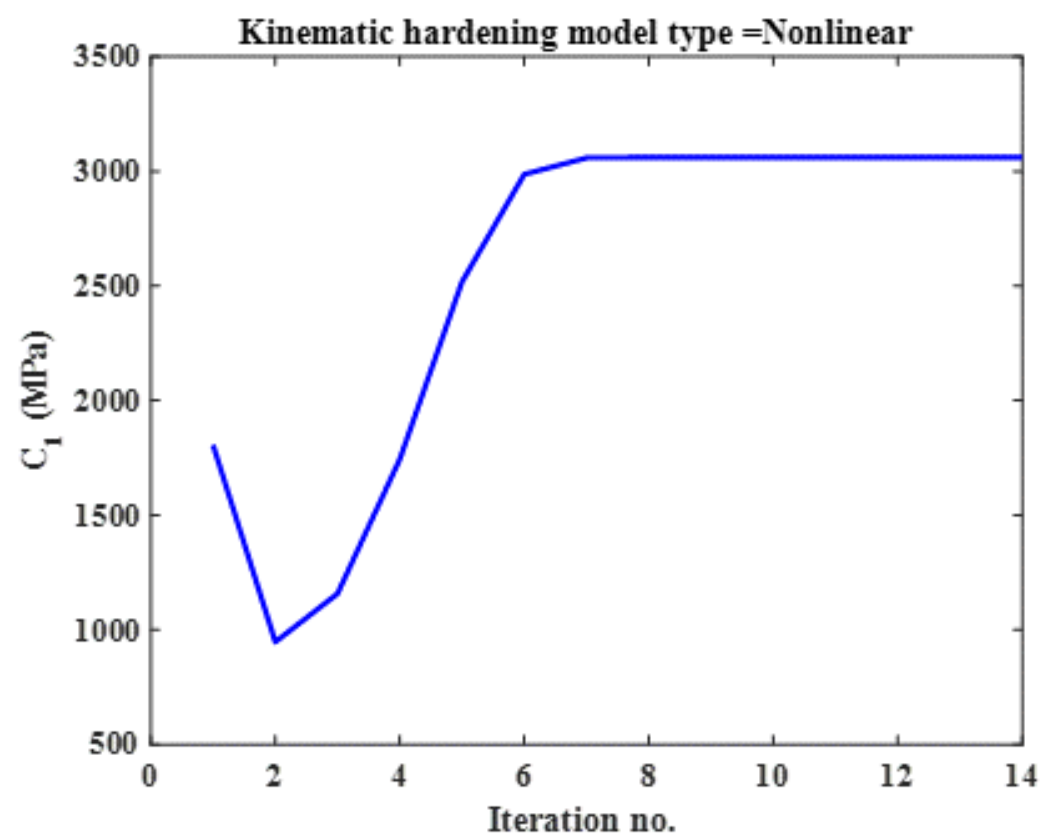

Figure 3A. 36 Nonlinear kinematic hardening constant $\mathrm{C} 1$ with respect to number of iteration during parameter estimation using gradient based optimization scheme and using ET-T04 stress-strain data (from $0.2 \%$ offset yield strain limit to $2 \%$ true total strain)

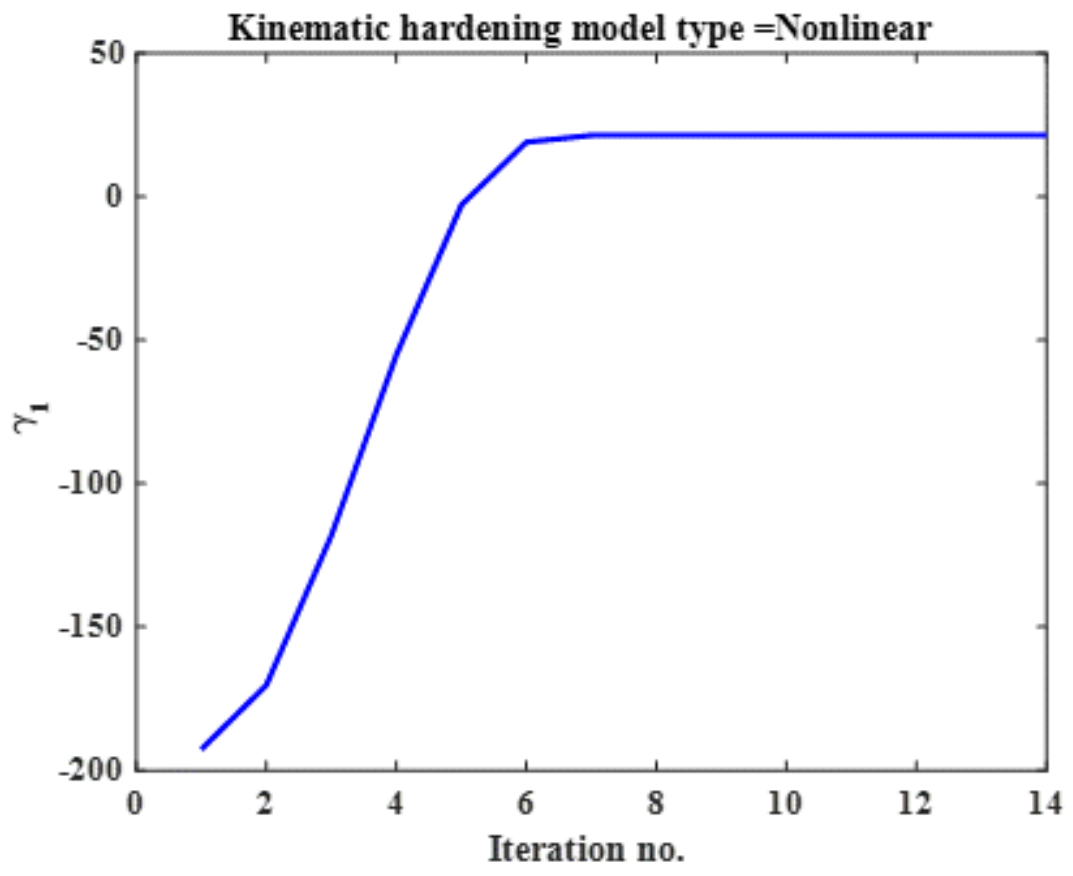

Figure 3A. 37 Nonlinear kinematic hardening constant $\gamma 1$ with respect to number of iteration during parameter estimation using gradient based optimization scheme and using ET-T04 stress-strain data (from $0.2 \%$ offset yield strain limit to $2 \%$ true total strain) 


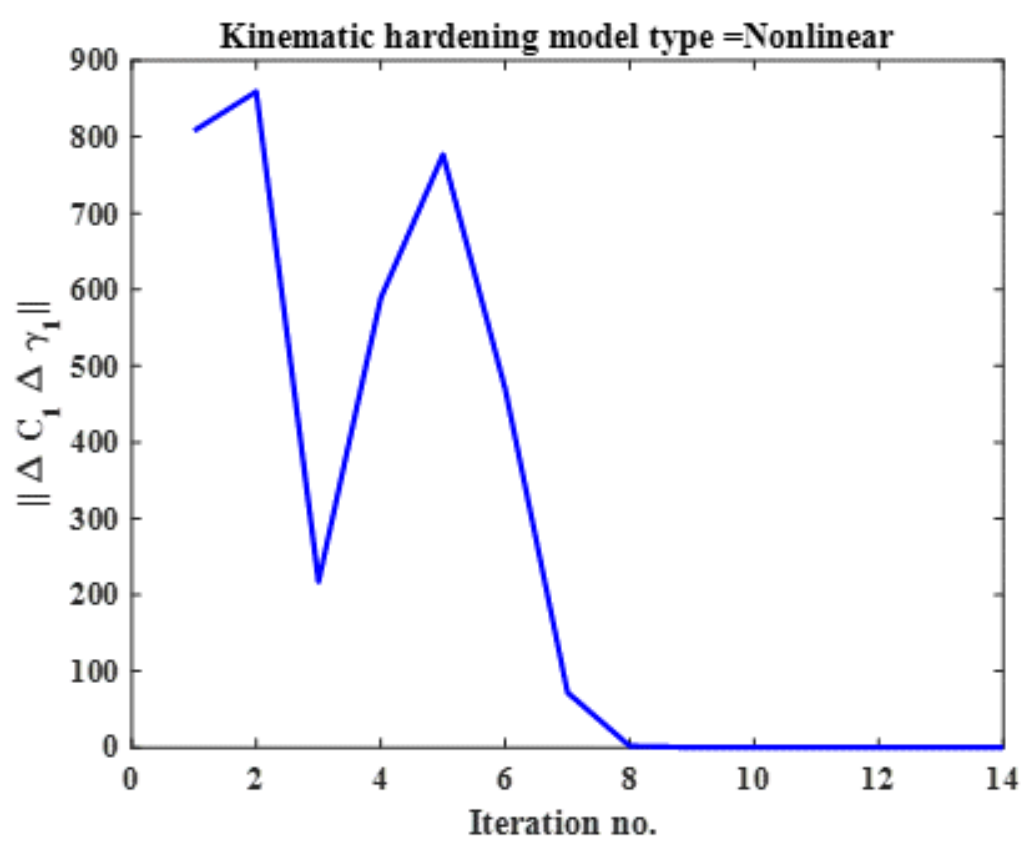

Figure 3A. $38 \mathrm{~L} 2$ norm (of incremental kinematic hardening constant $\mathrm{C} 1$ and $\gamma 1$ ) with respect to number of iteration during parameter estimation using gradient based optimization scheme and using ET-T04 stress-strain data (from $0.2 \%$ offset yield strain limit to $2 \%$ true total strain).

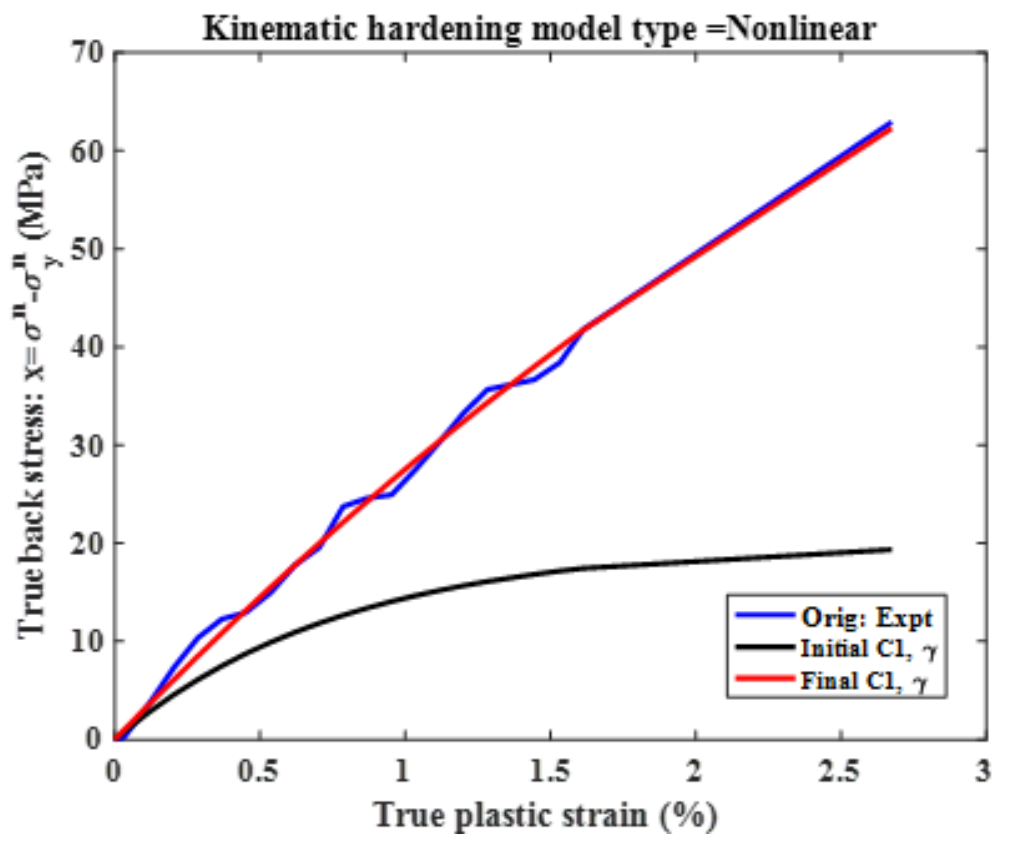

Figure 3A. 39 Comparison of predicted true back stress (using nonlinear kinematic hardening parameter $\mathrm{C} 1$ and $\gamma 1$ and considering $0.2 \%$ offset yield strain as yield limit) with experimental true back stress for ET-T04 tensile test. 


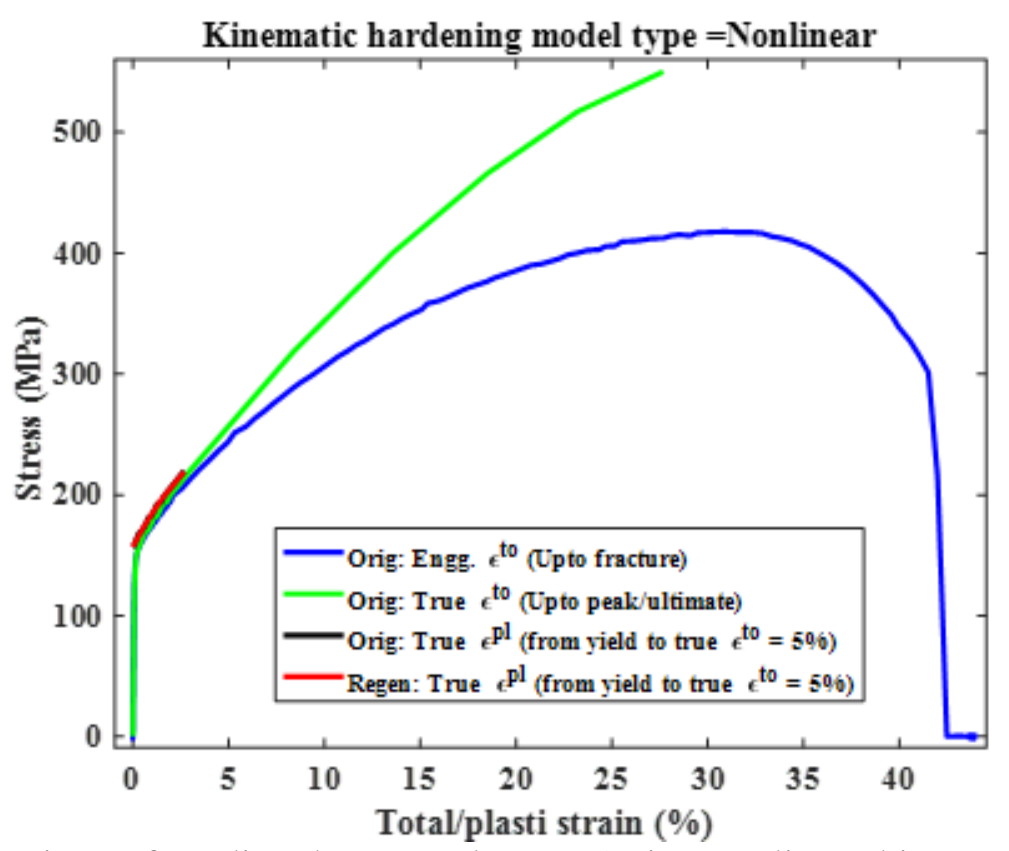

Figure 3A. 40 Comparison of predicted true total stress (using nonlinear kinematic hardening parameter $\mathrm{C} 1$ and $\gamma 1$ and considering $0.2 \%$ offset yield strain as yield limit) with experimental true total stress for ET-T04 tensile test. 


\section{Appendix-3B: Supplementary Results for ET-F06 Fatigue Test}

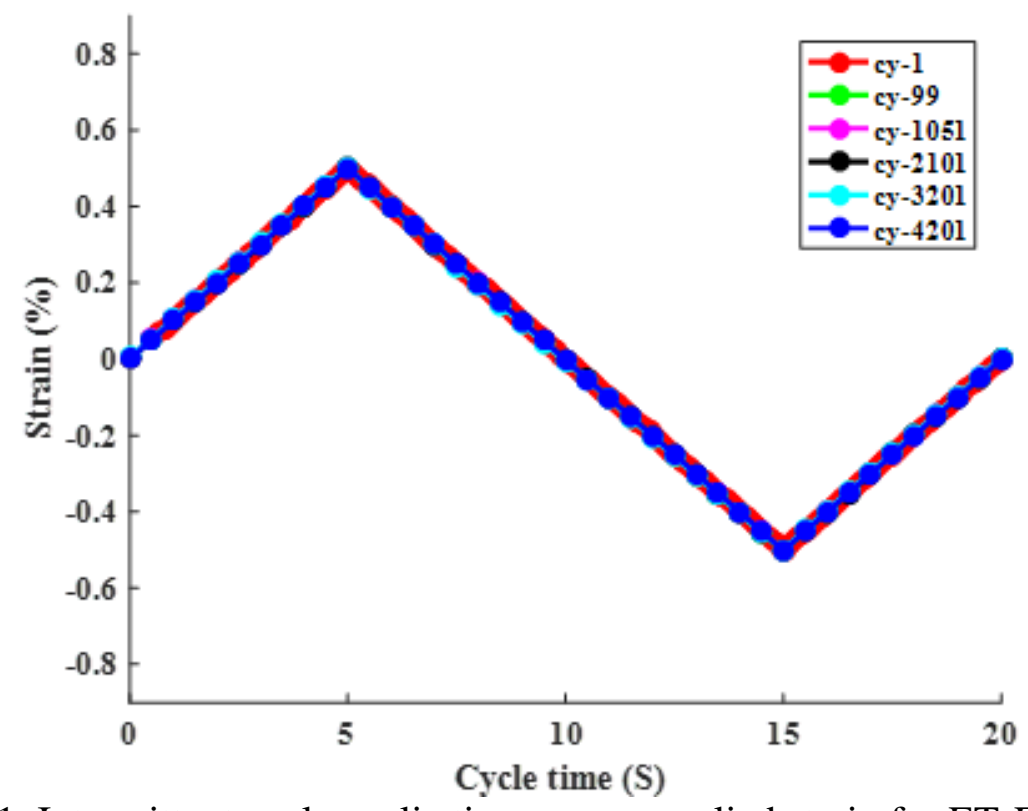

Figure 3B. 1 Intermittent cycle cyclic time versus applied strain for ET-F06 fatigue test.

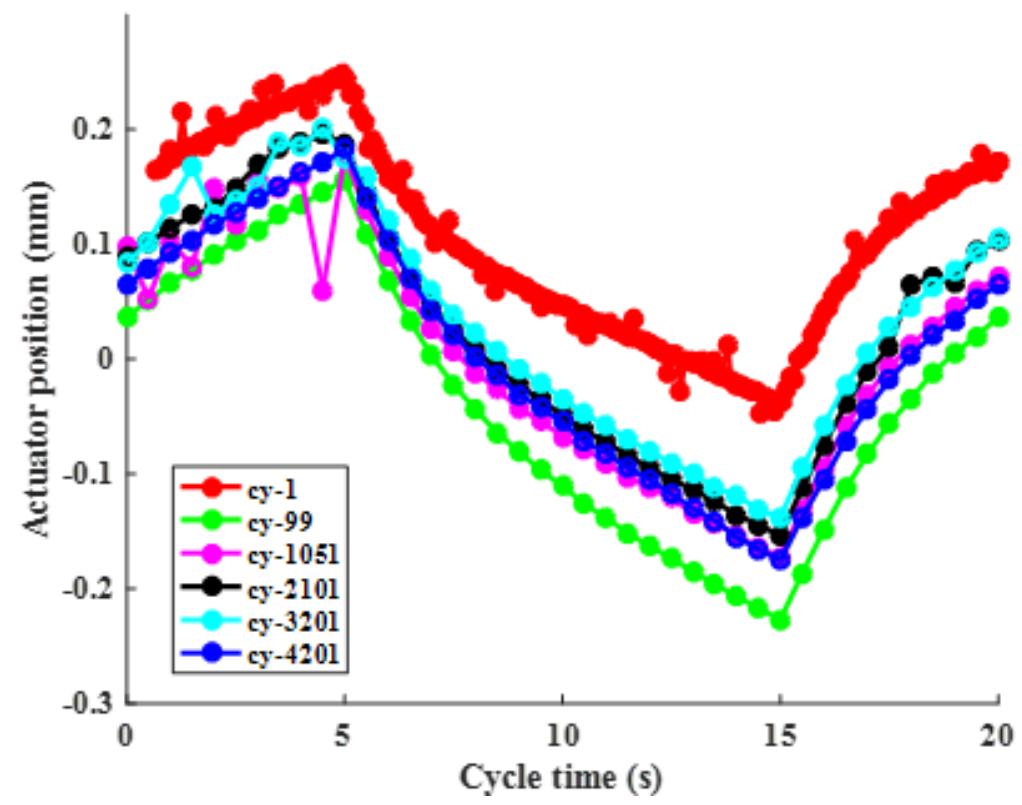

Figure 3B. 2 Intermittent cycle cyclic time versus observed frame actuator position (baseline removed) for ET-F06 fatigue test. 


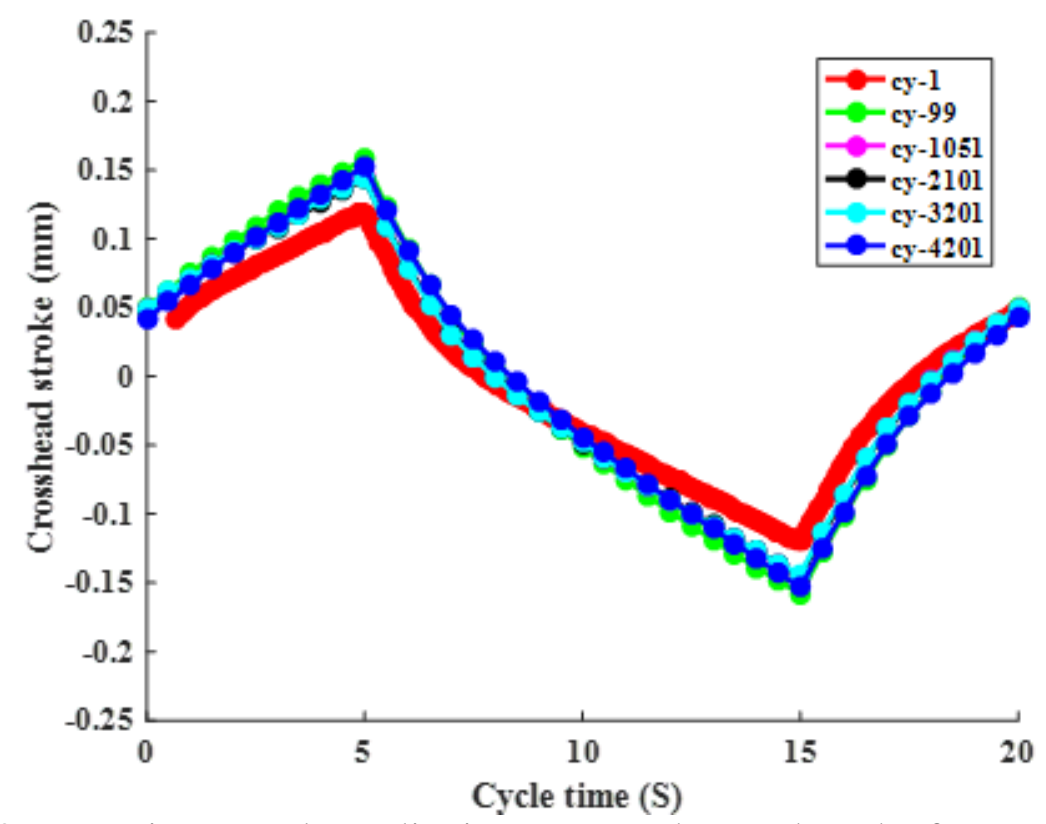

Figure 3B. 3 Intermittent cycle cyclic time versus observed stroke for ET-F06 fatigue test.

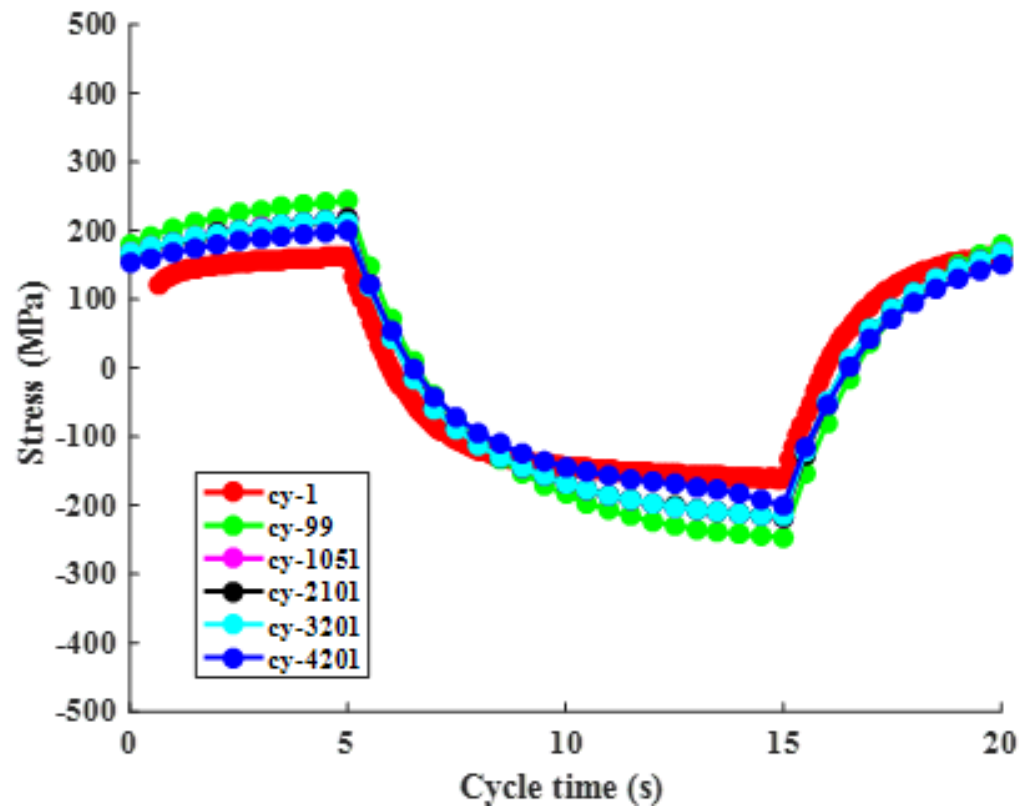

Figure 3B. 4 Intermittent cycle cyclic time versus observed stress for ET-F06 fatigue test. 


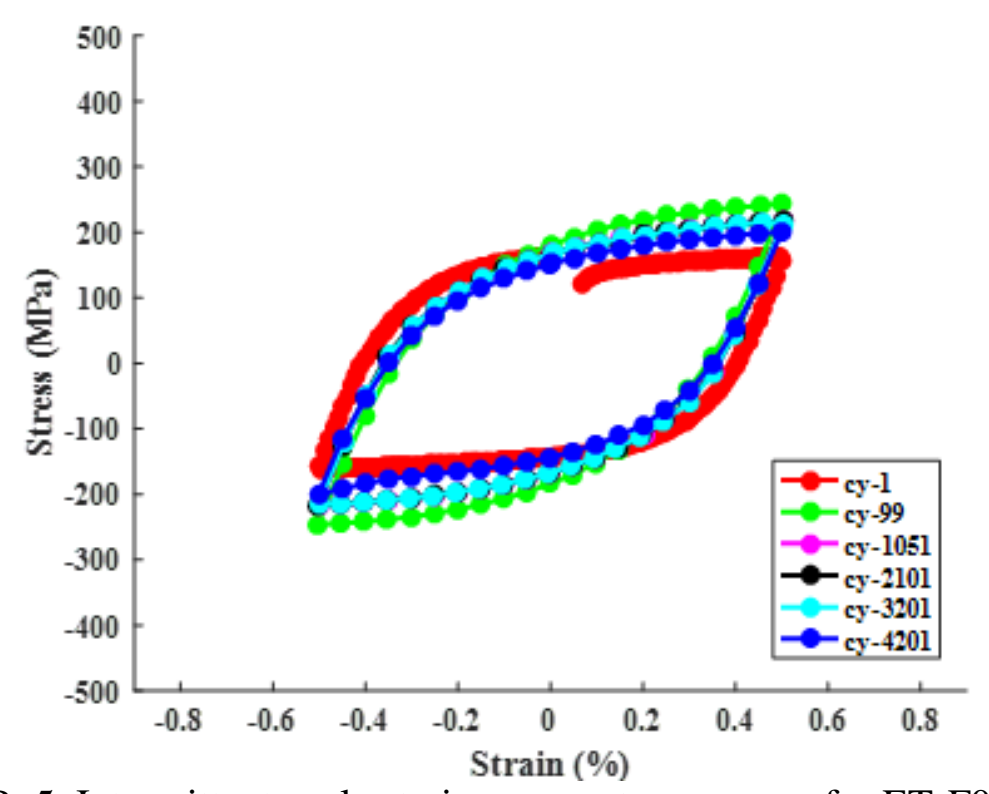

Figure 3B. 5 Intermittent cycle strain versus stress curves for ET-F06 fatigue test.

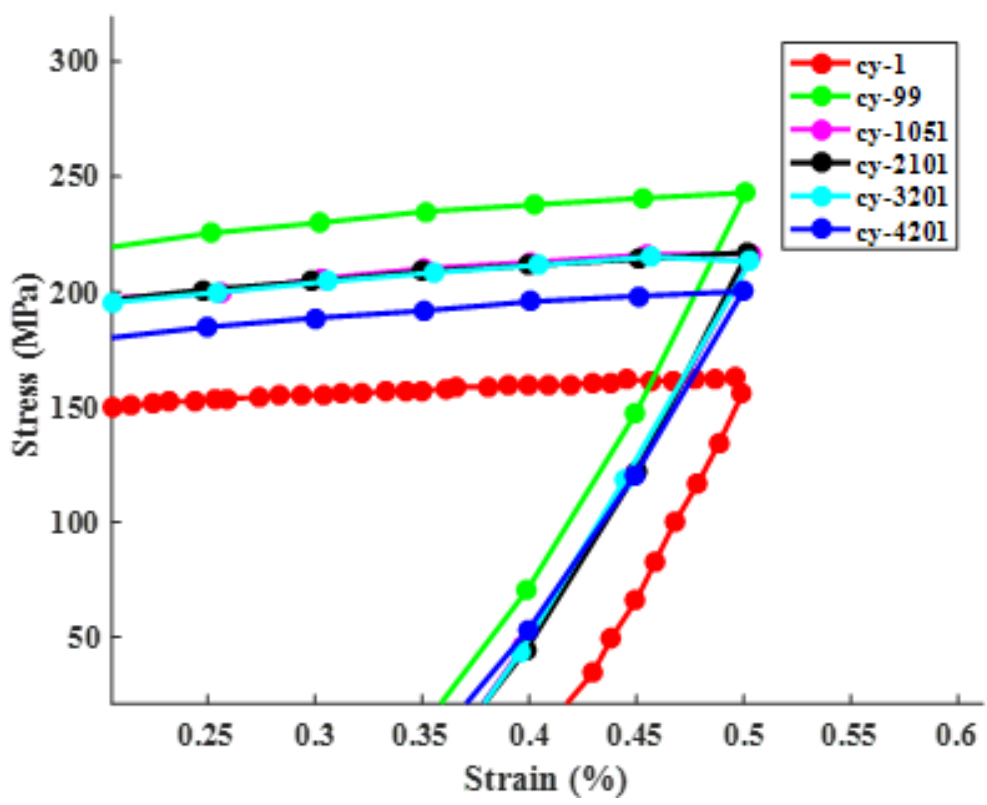

Figure 3B. 6 Magnified figure of Figure 3B. 5. 


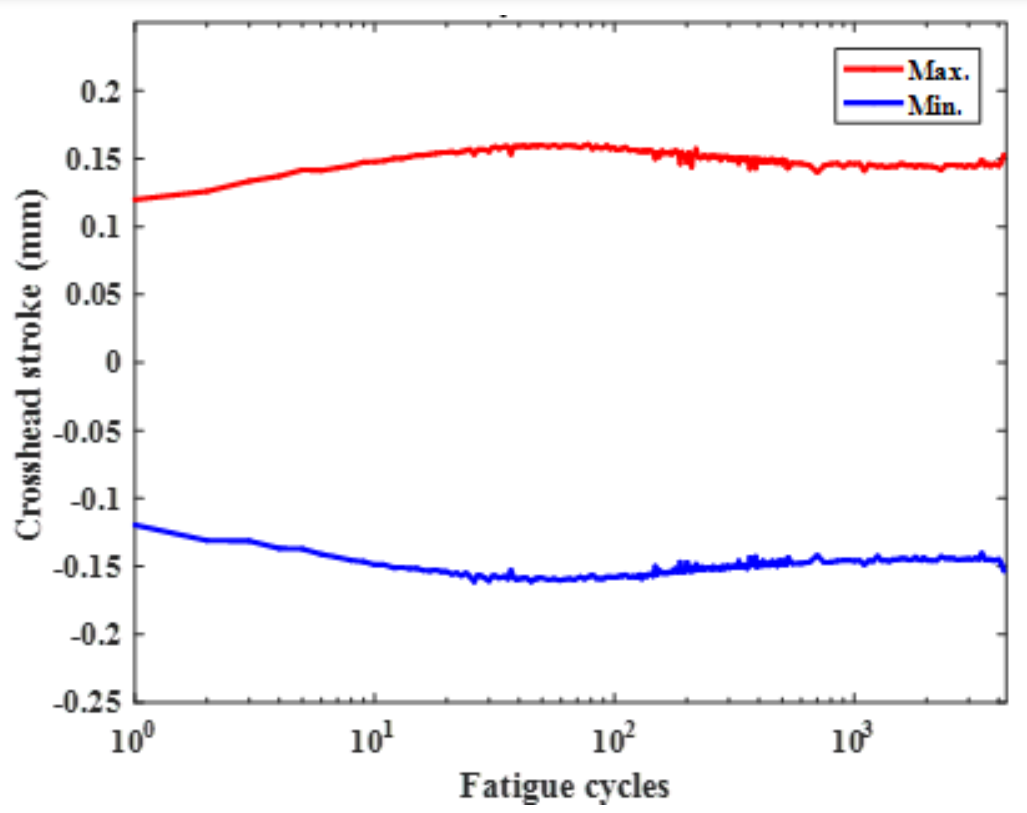

Figure 3B. 7 Maximum and minimum observed stroke during ET-F06.

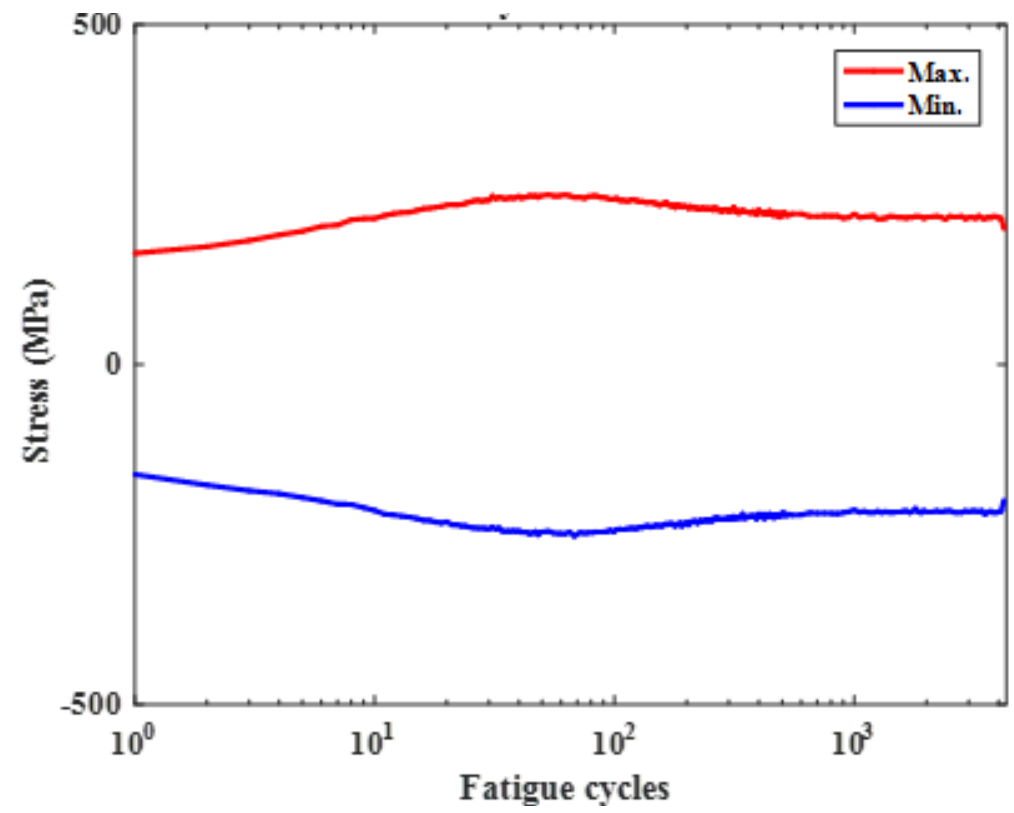

Figure 3B. 8 Maximum and minimum observed stress during ET-F06. 


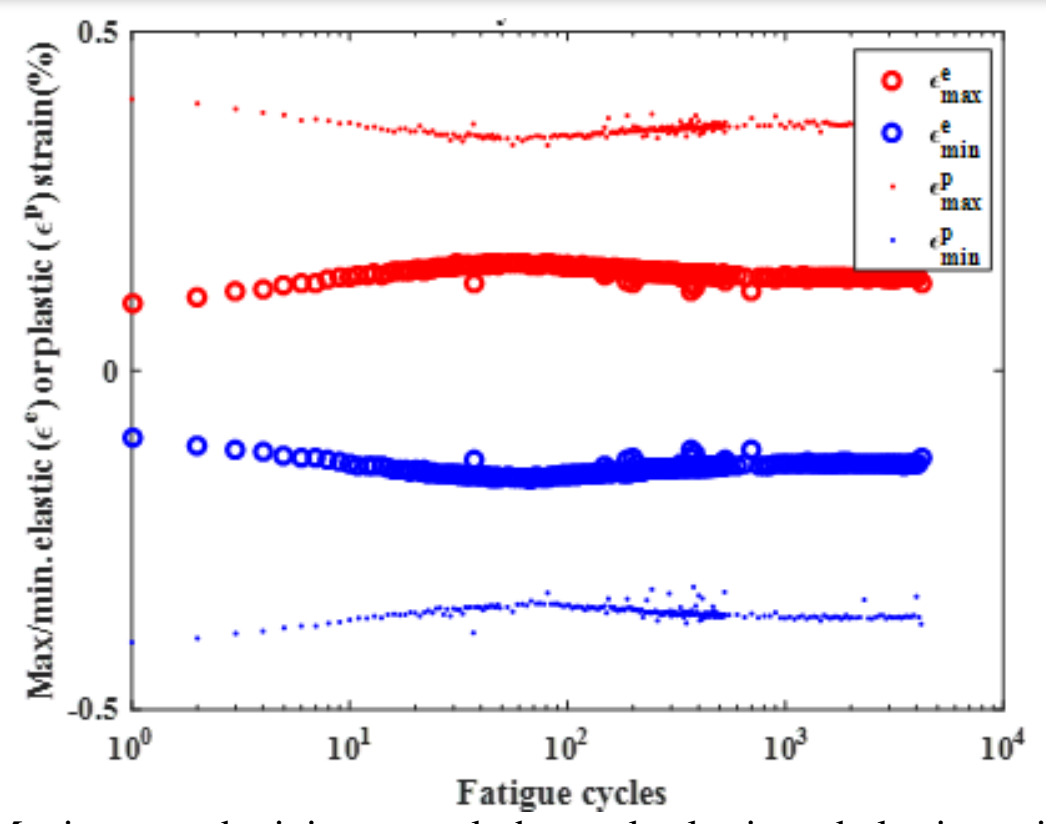

Figure 3B. 9 Maximum and minimum cycle-by-cycle elastic and plastic strain during ET-F06.

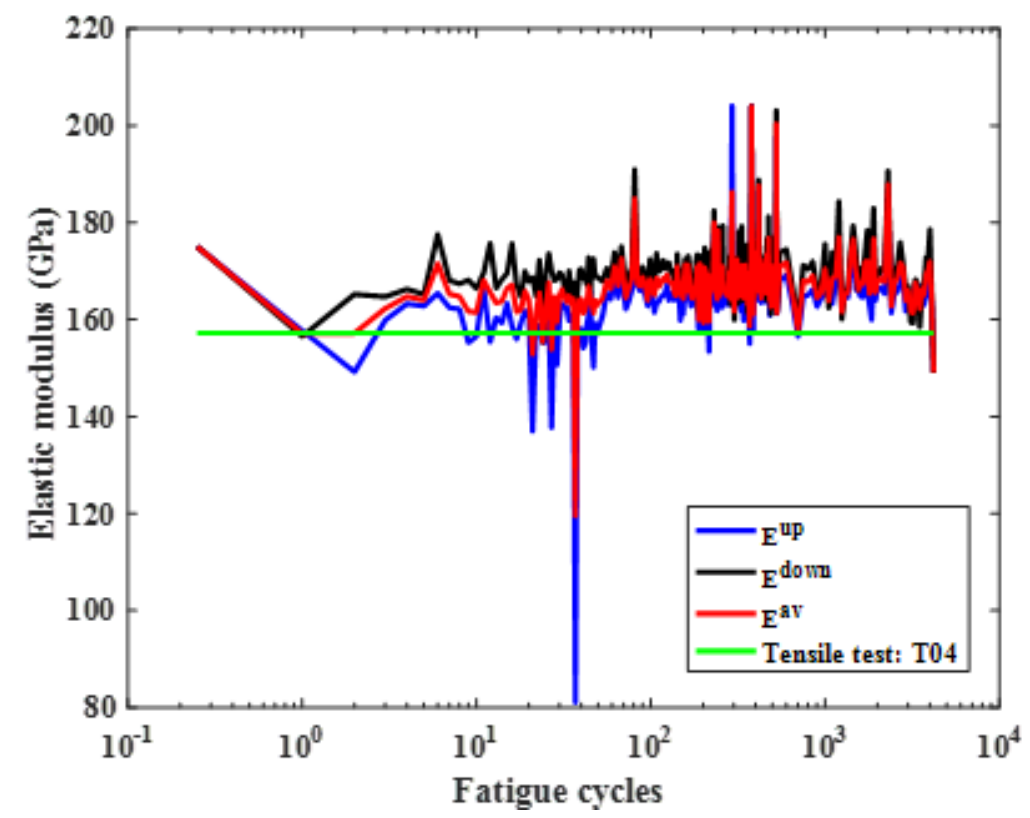

Figure 3B. 10 Fatigue cycle versus estimated elastic modulus for ET-F06 fatigue test. Also the figure shows the comparison with respect to corresponding tensile test (ET-T04) elastic modulus. 


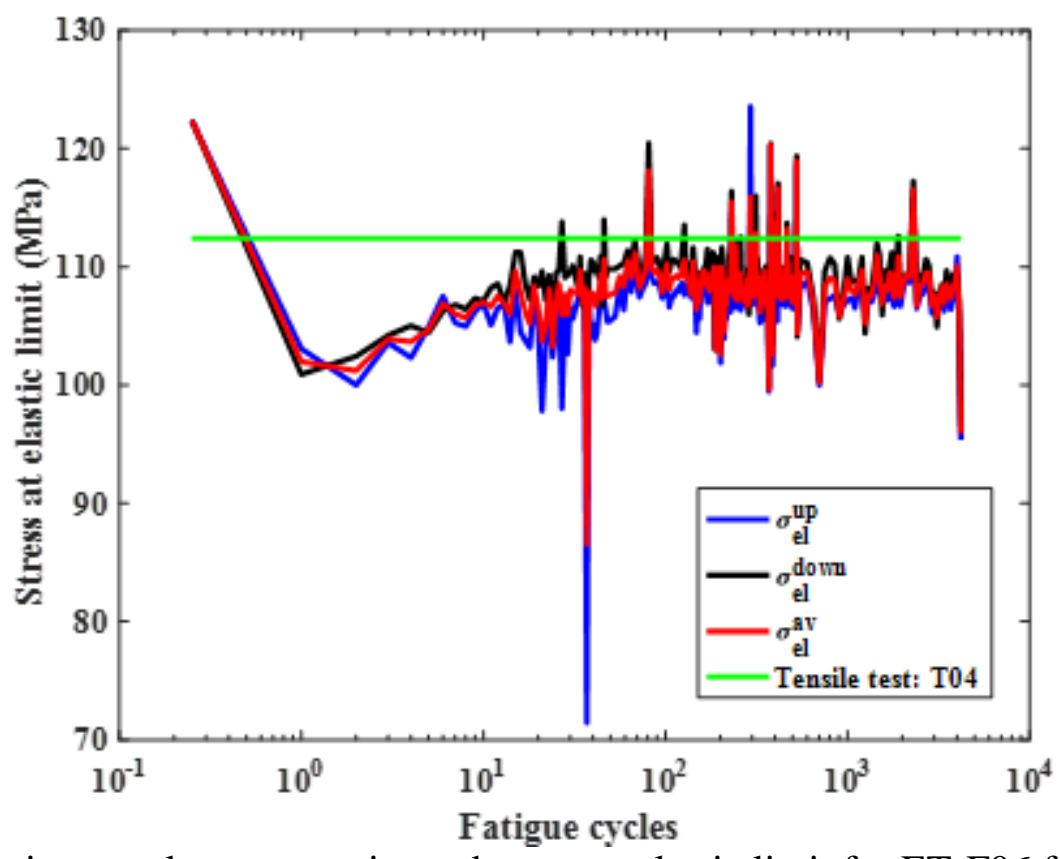

Figure 3B. 11 Fatigue cycle versus estimated stress at elastic limit for ET-F06 fatigue test. Also the figure shows the comparison with respect to corresponding tensile test (ET-T04) elastic limit stress.

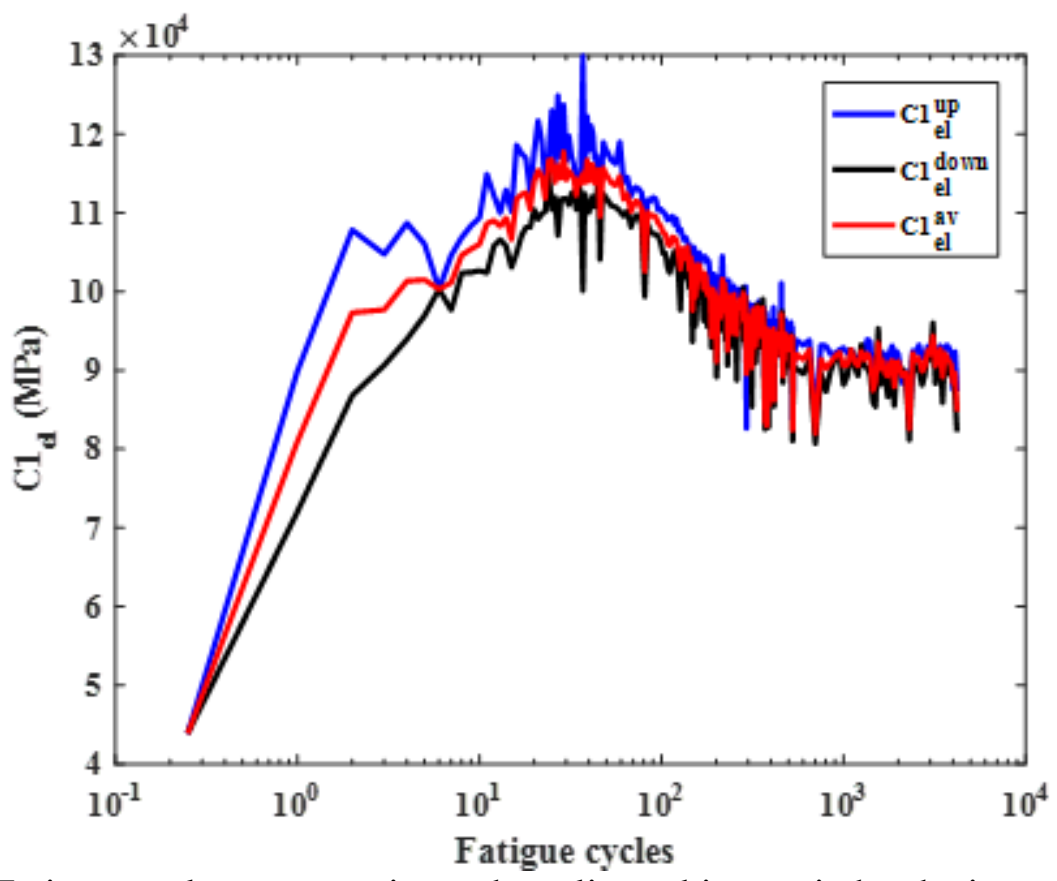

Figure 3B. 12 Fatigue cycle versus estimated nonlinear kinematic hardening or Chaboche model parameters $\left(\mathrm{C} 1_{\mathrm{el}}\right)$ for ET-F06 fatigue test, with cyclic elastic limit stress was considered to estimate the corresponding cycle back stress. 


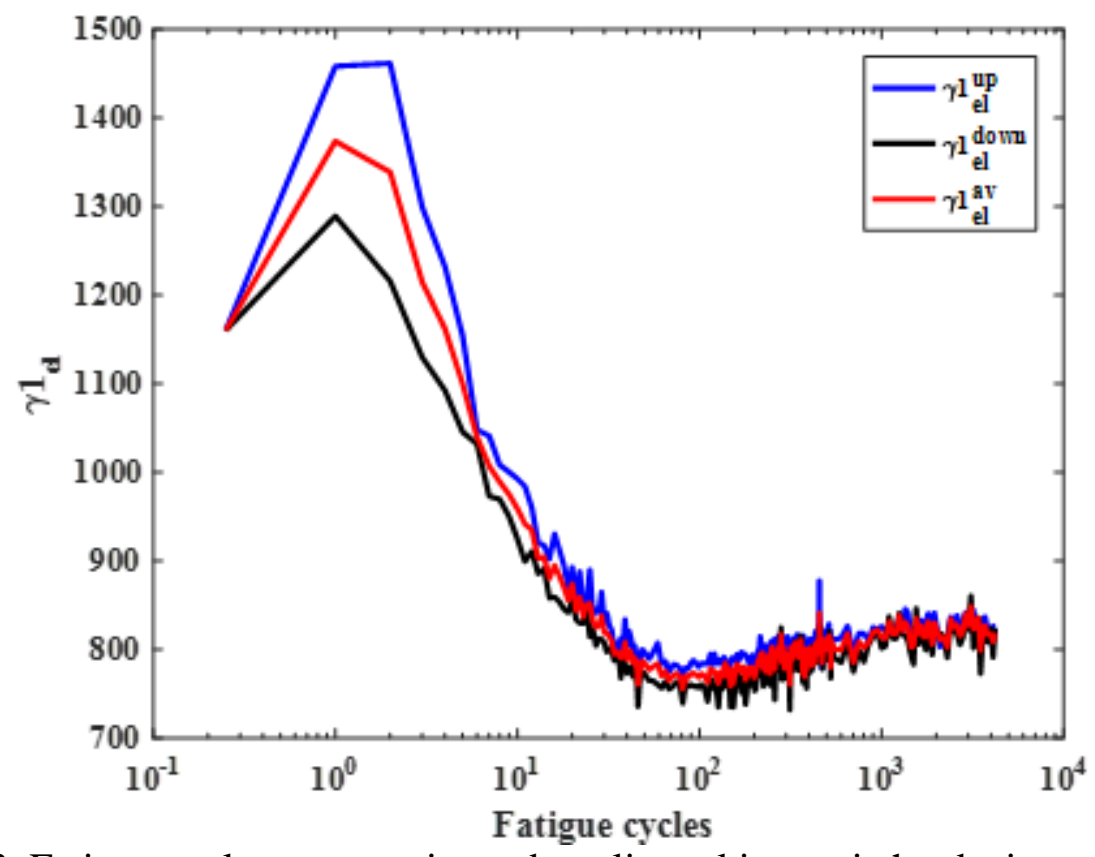

Figure 3B. 13 Fatigue cycle versus estimated nonlinear kinematic hardening or Chaboche model parameters $\left(\gamma 1_{\mathrm{el}}\right)$ for ET-F06 fatigue test, with cyclic elastic limit stress was considered to estimate the corresponding cycle back stress.

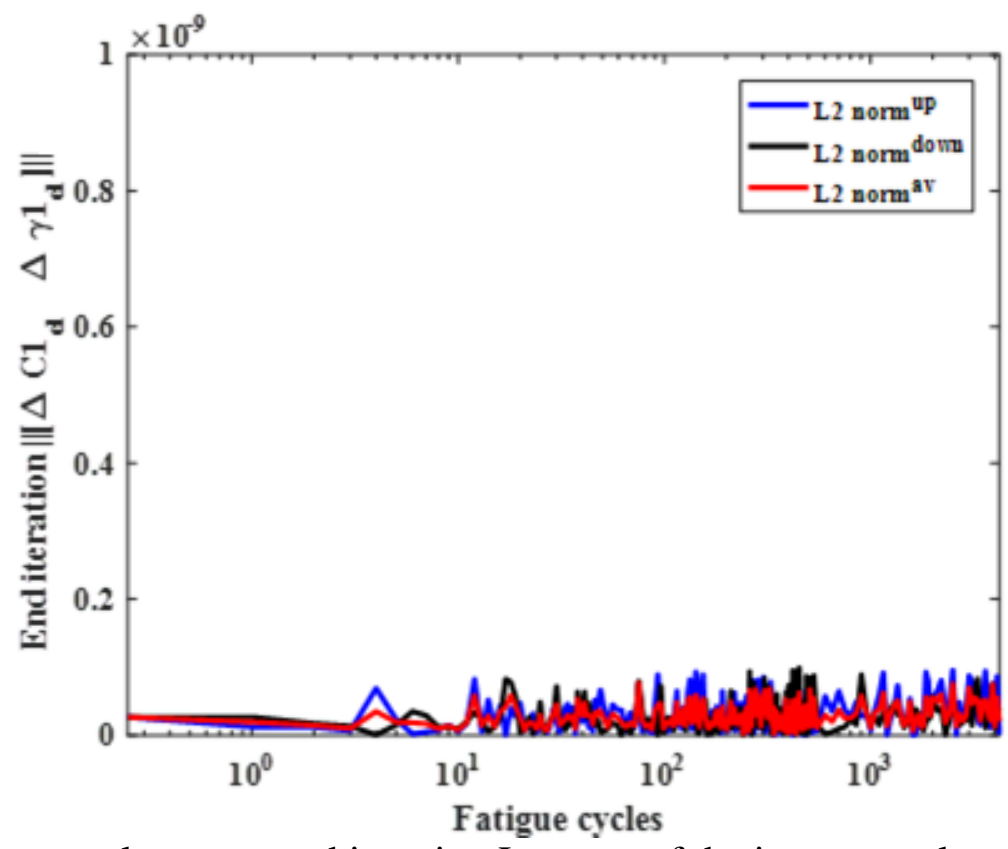

Figure 3B. 14 Fatigue cycle versus end iteration $\mathrm{L}_{2}$ norm of the incremental nonlinear Chaboche model parameters $\left(\mathrm{C} 1_{\mathrm{el}}\right.$ and $\left.\gamma 1_{\mathrm{el}}\right)$ for ET-F06 fatigue test, with cyclic elastic limit stress was considered to estimate the corresponding cycle back stress. 


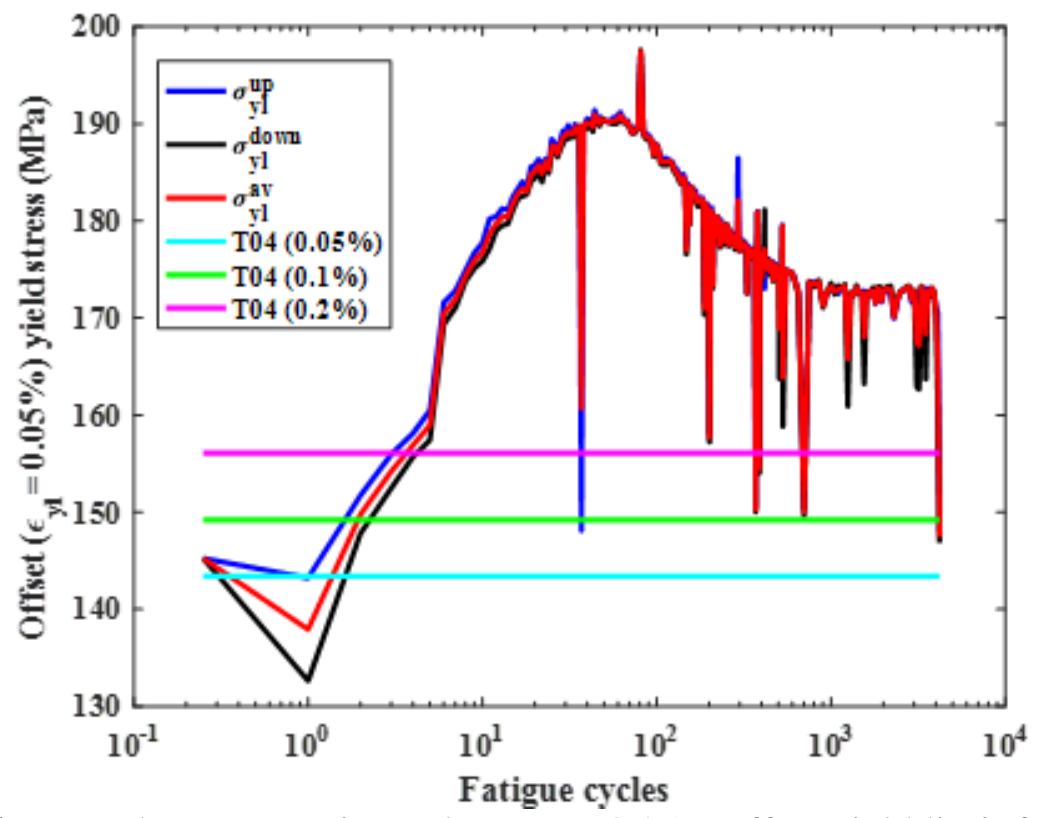

Figure 3B. 15 Fatigue cycle versus estimated stress at $0.05 \%$ offset yield limit for ET-F06 fatigue test. Also the figure shows the comparison with respect to corresponding tensile test (ET-T04) yield limit stresses.

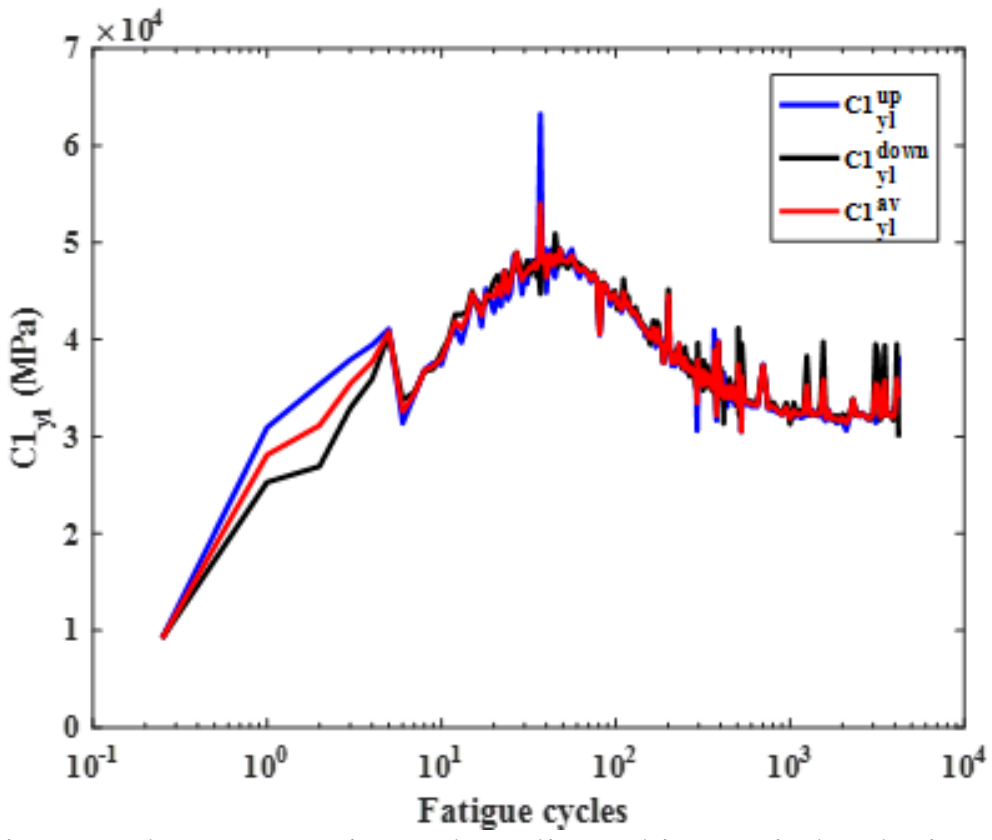

Figure 3B. 16 Fatigue cycle versus estimated nonlinear kinematic hardening or Chaboche model parameter $\left(\mathrm{C}_{\mathrm{yl}}\right)$ for ET-F06 fatigue test, with cyclic $0.05 \%$ offset yield limit stress was considered to estimate the corresponding cycle back stress. 


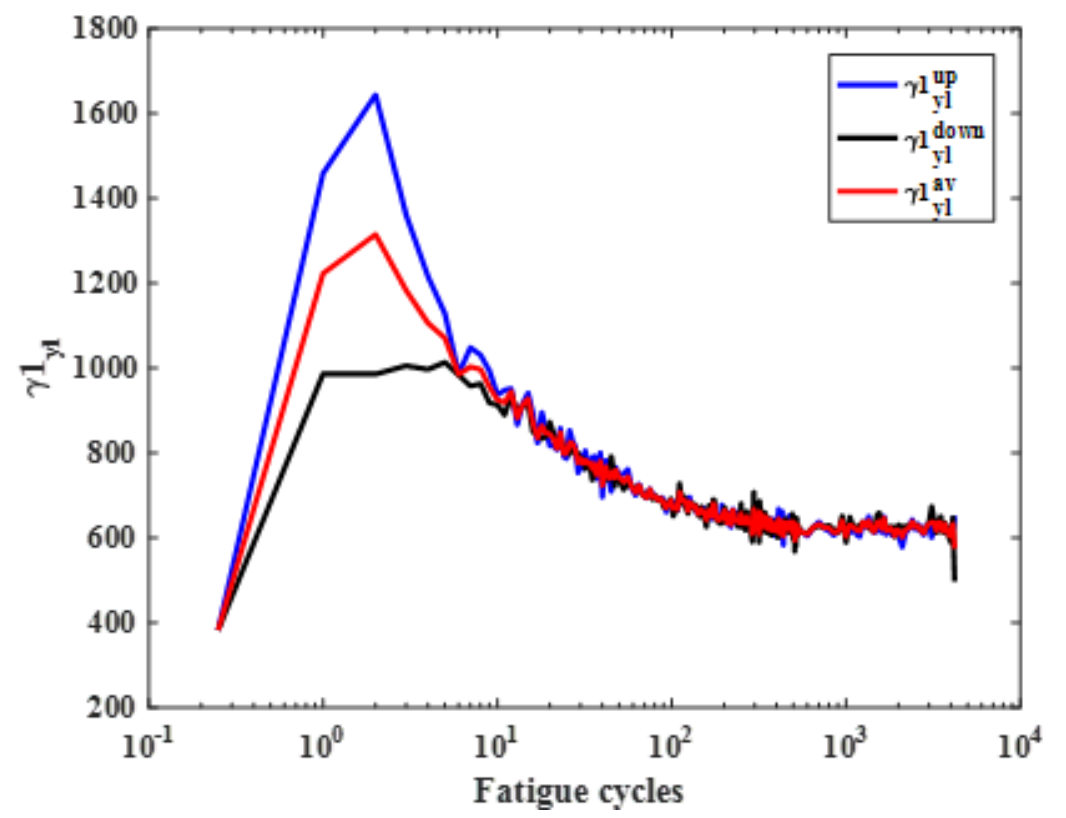

Figure 3B. 17 Fatigue cycle versus estimated nonlinear kinematic hardening or Chaboche model parameter $\left(\gamma 1_{\mathrm{yl}}\right)$ for ET-F06 fatigue test, with cyclic $0.05 \%$ offset yield limit stress was considered to estimate the corresponding cycle back stress.

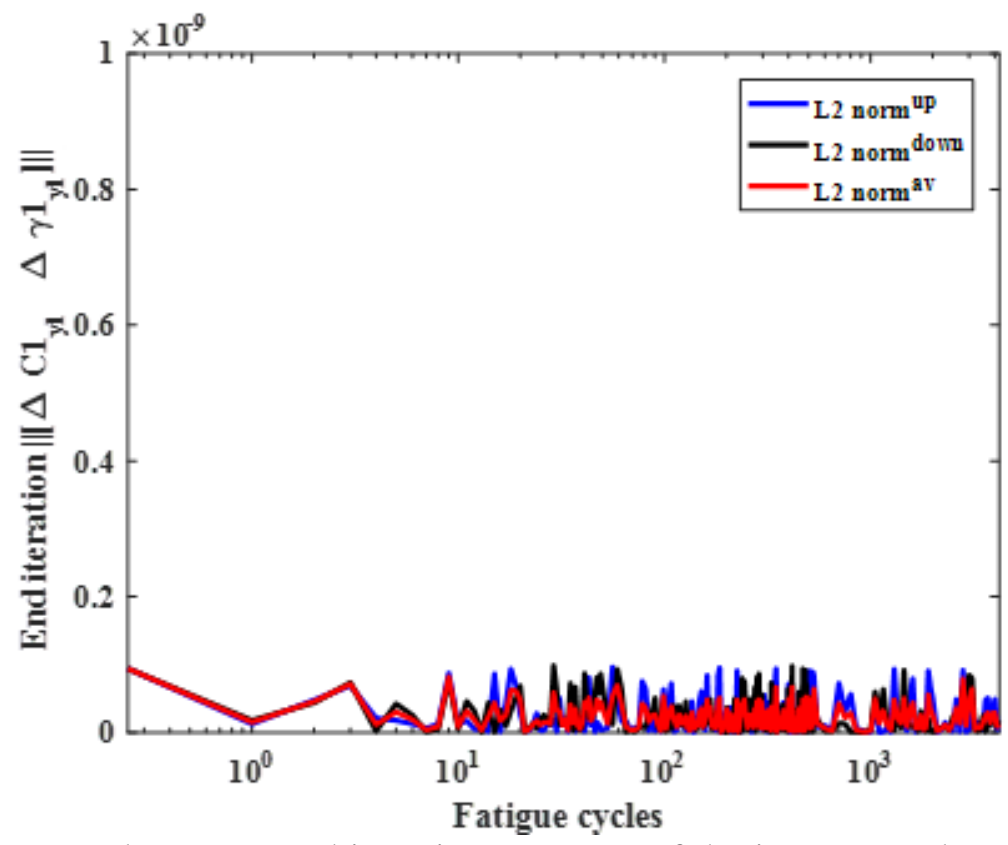

Figure 3B. 18 Fatigue cycle versus end iteration $\mathrm{L}_{2}$ norm of the incremental nonlinear Chaboche model parameters $\left(\mathrm{C}_{\mathrm{yl}}, \gamma 1_{\mathrm{yl}}\right)$ for ET-F06 fatigue test, with cyclic $0.05 \%$ offset yield limit stress was considered to estimate the corresponding cycle back stress. 


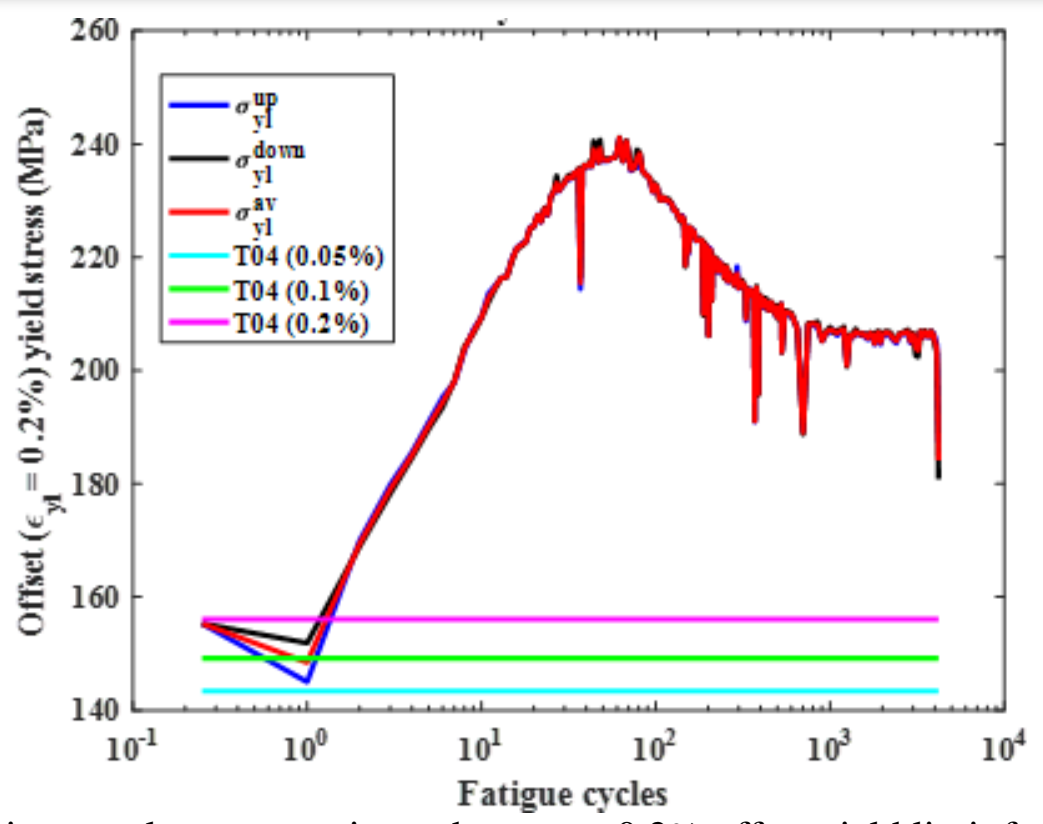

Figure 3B. 19 Fatigue cycle versus estimated stress at $0.2 \%$ offset yield limit for ET-F06 fatigue test. Also the figure shows the comparison with respect to corresponding tensile test (ET-T04) yield limit stresses.

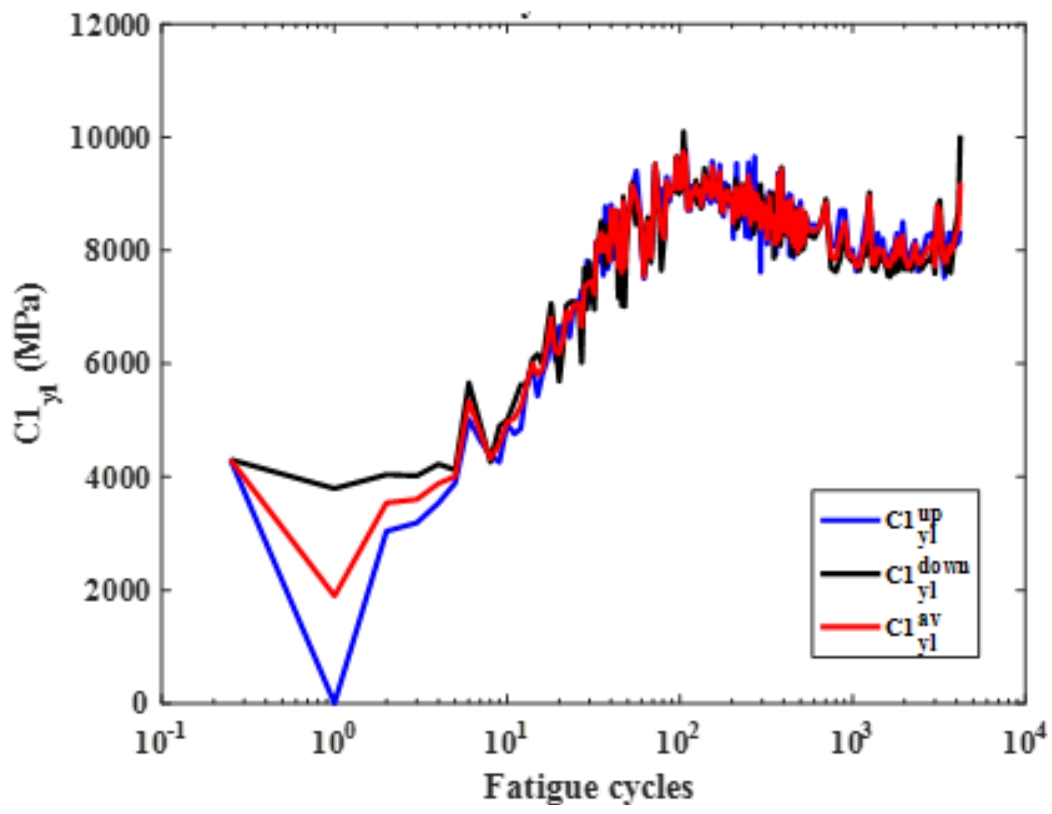

Figure 3B. 20 Fatigue cycle versus estimated linear kinematic hardening or Chaboche model parameter $\left(\mathrm{C}_{\mathrm{yl}}\right)$ for ET-F06 fatigue test, with cyclic $0.2 \%$ offset yield limit stress was considered to estimate the corresponding cycle back stress. 


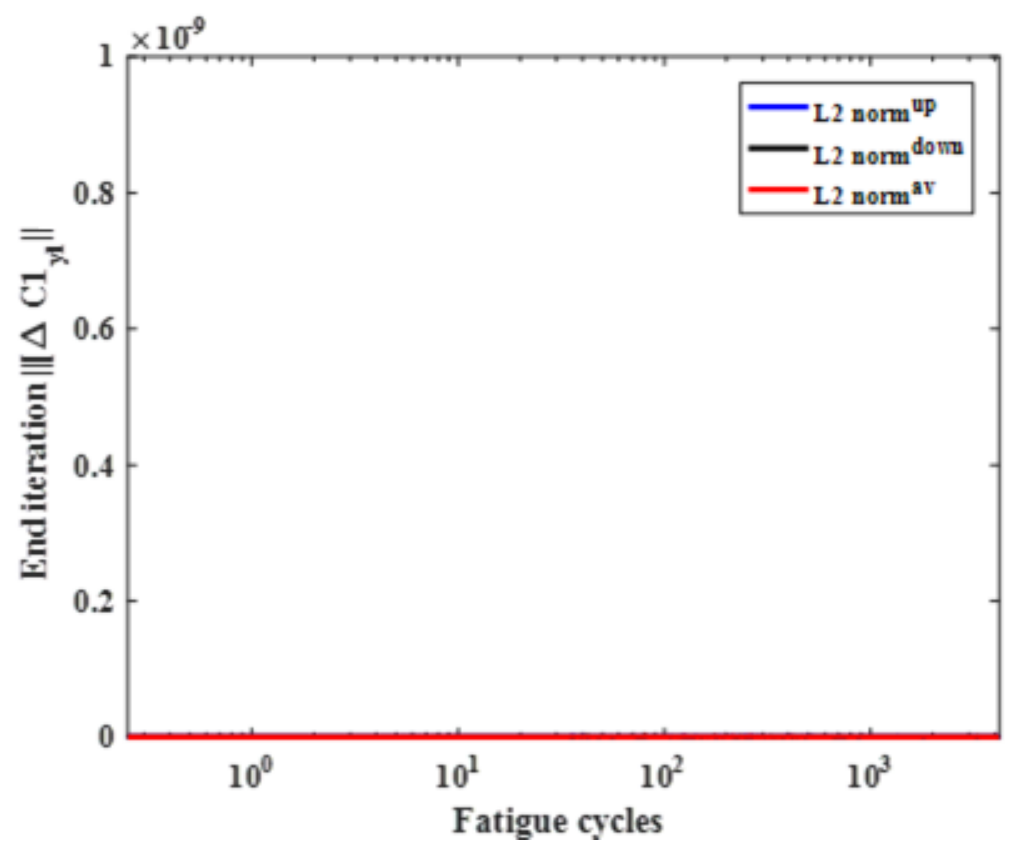

Figure 3B. 21 Fatigue cycle versus end iteration $\mathrm{L}_{2}$ norm of the incremental linear Chaboche model parameter $\left(\mathrm{C}_{\mathrm{yl}}\right)$ for ET-F06 fatigue test, with cyclic $0.2 \%$ offset yield limit stress was considered to estimate the corresponding cycle back stress.

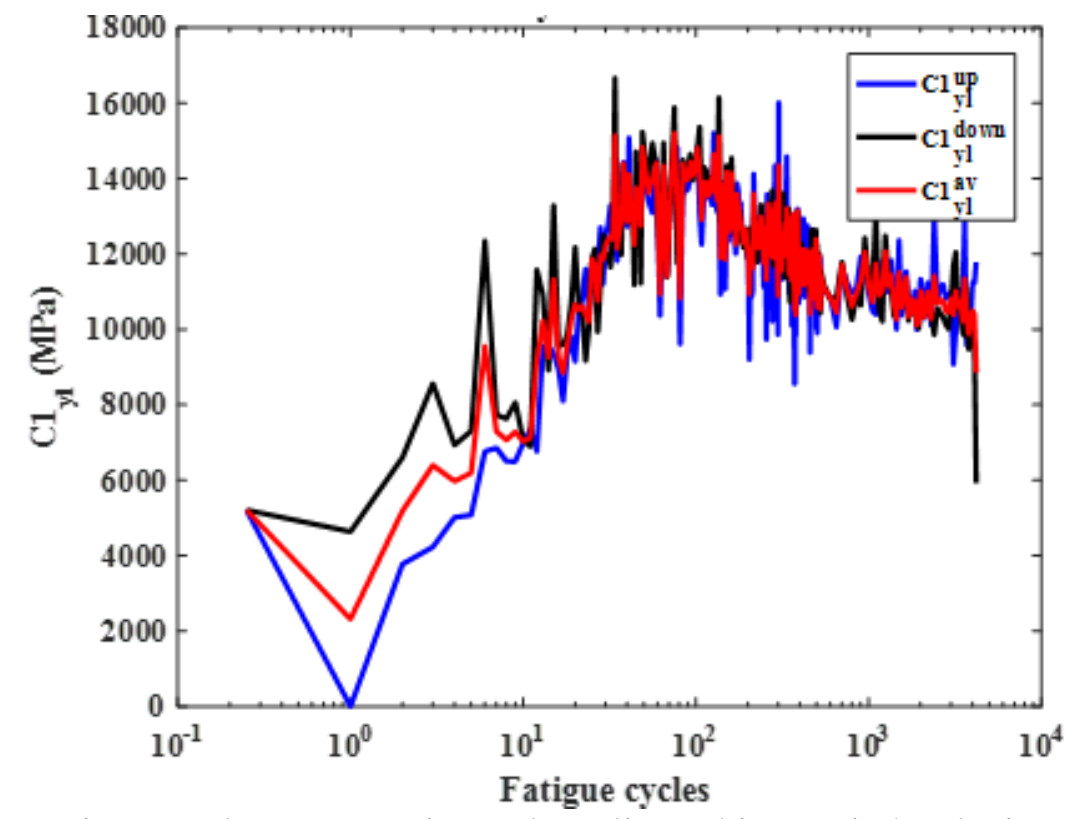

Figure 3B. 22 Fatigue cycle versus estimated nonlinear kinematic hardening or Chaboche model parameter $\left(\mathrm{Cl}_{\mathrm{yl}}\right)$ for ET-F06 fatigue test, with cyclic $0.2 \%$ offset yield limit stress was considered to estimate the corresponding cycle back stress. 


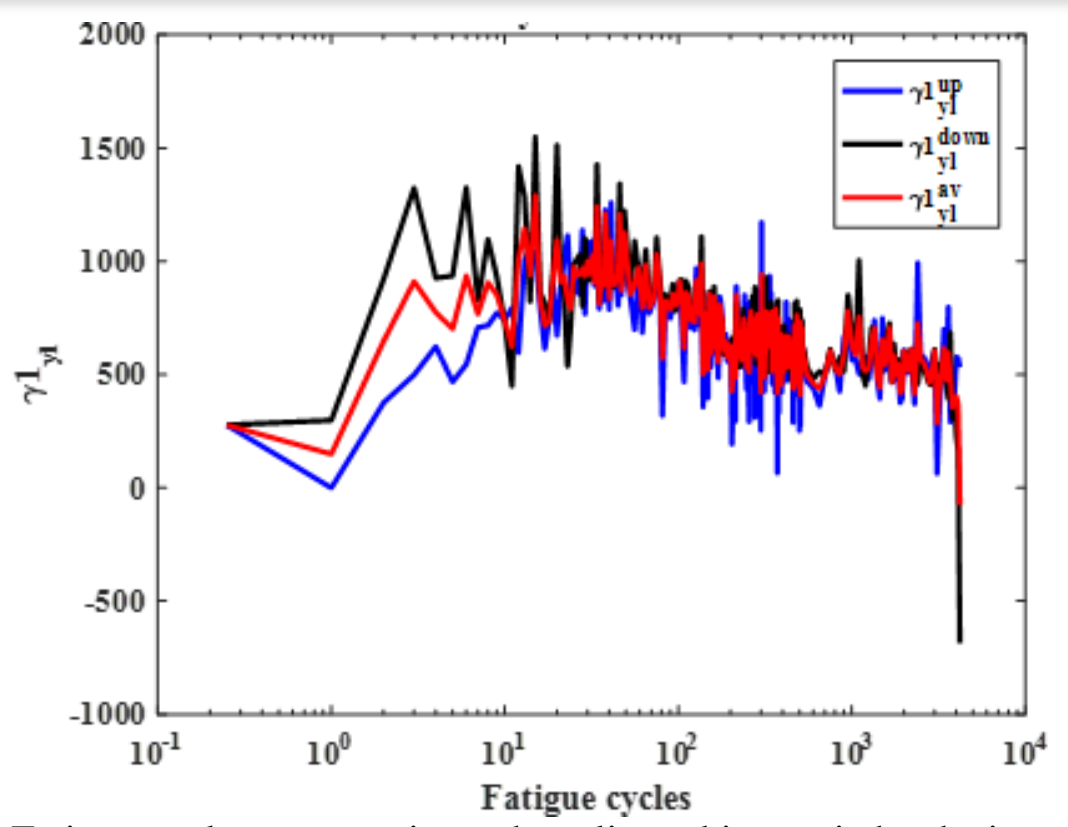

Figure 3B. 23 Fatigue cycle versus estimated nonlinear kinematic hardening or Chaboche model parameter $\left(\gamma 1_{\mathrm{yl}}\right)$ for ET-F06 fatigue test, with cyclic $0.2 \%$ offset yield limit stress was considered to estimate the corresponding cycle back stress.

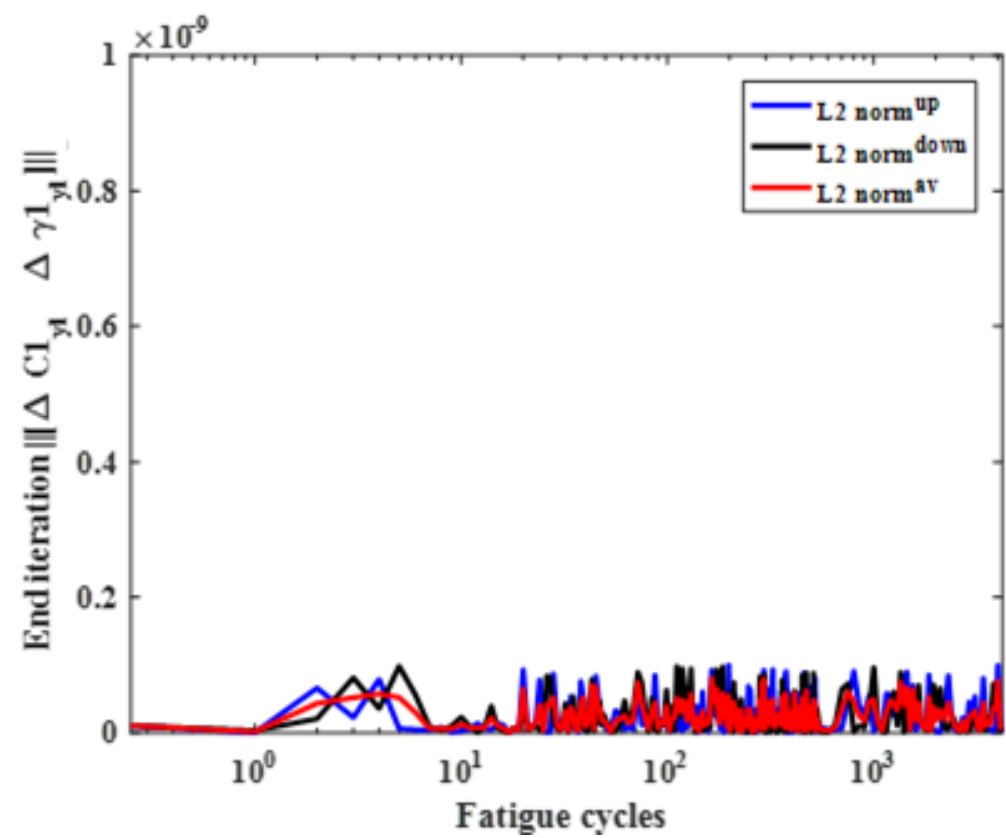

Figure 3B. 24 Fatigue cycle versus end iteration $\mathrm{L}_{2}$ norm of the incremental nonlinear Chaboche model parameters $\left(\mathrm{C}_{\mathrm{yl}}, \gamma 1_{\mathrm{yl}}\right)$ for ET-F06 fatigue test, with cyclic $0.2 \%$ offset yield limit stress was considered to estimate the corresponding cycle back stress. 


\section{Appendix-3C: Supplementary Results for ET-F41 Fatigue Test}

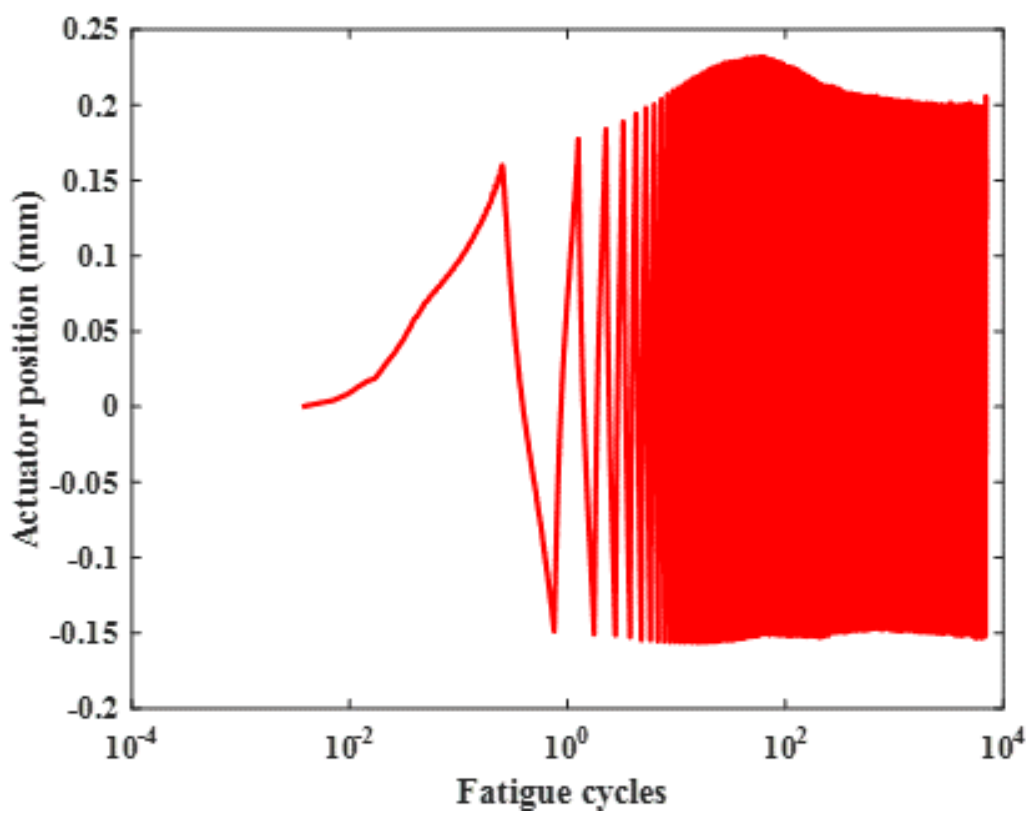

Figure 3C. 1 Observed actuator position during ET-F41.

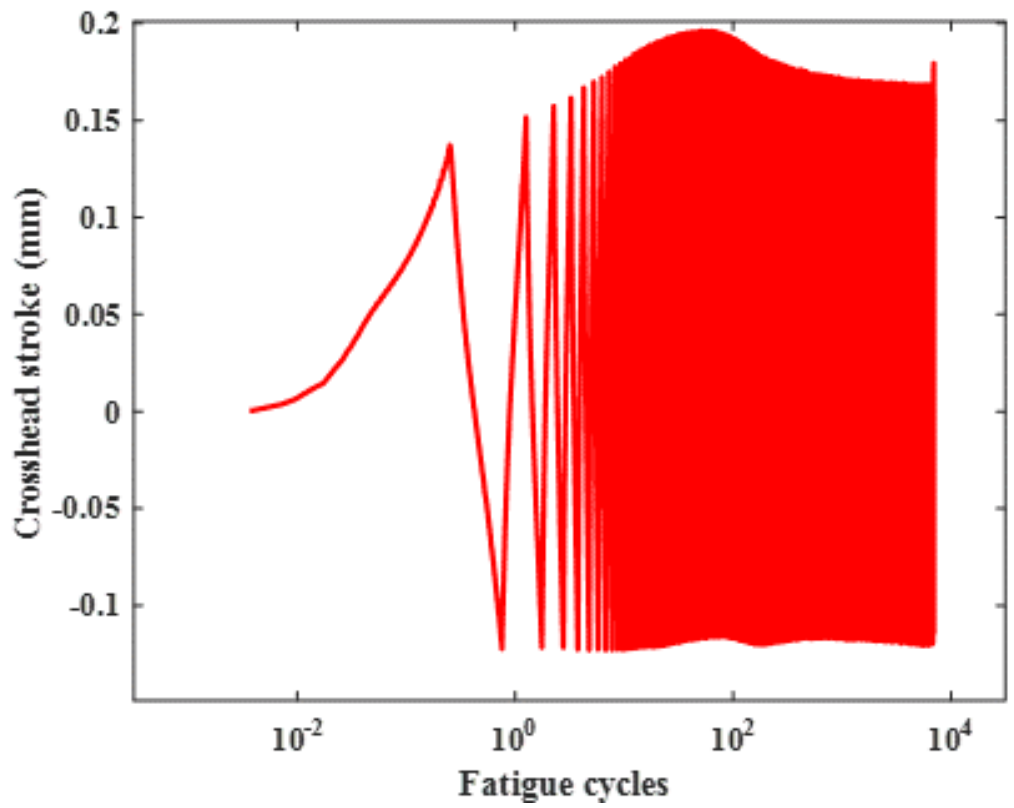

Figure 3C. 2 Observed stroke during ET-F41. 


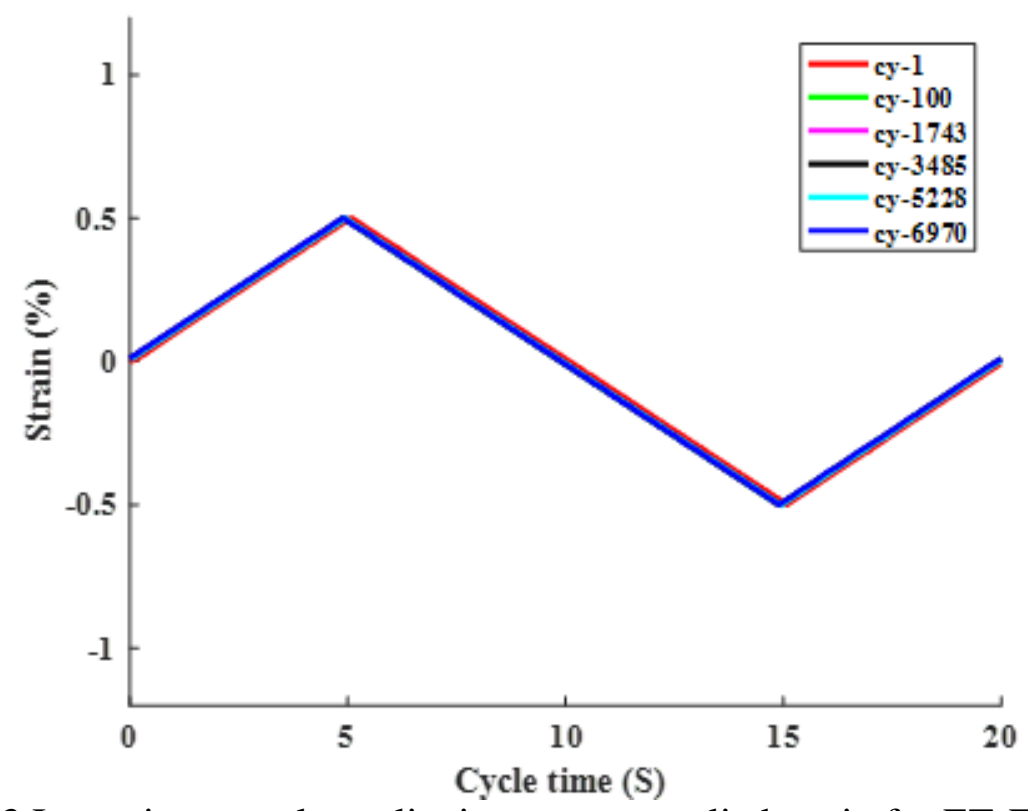

Figure 3C. 3 Intermittent cycle cyclic time versus applied strain for ET-F41 fatigue test.

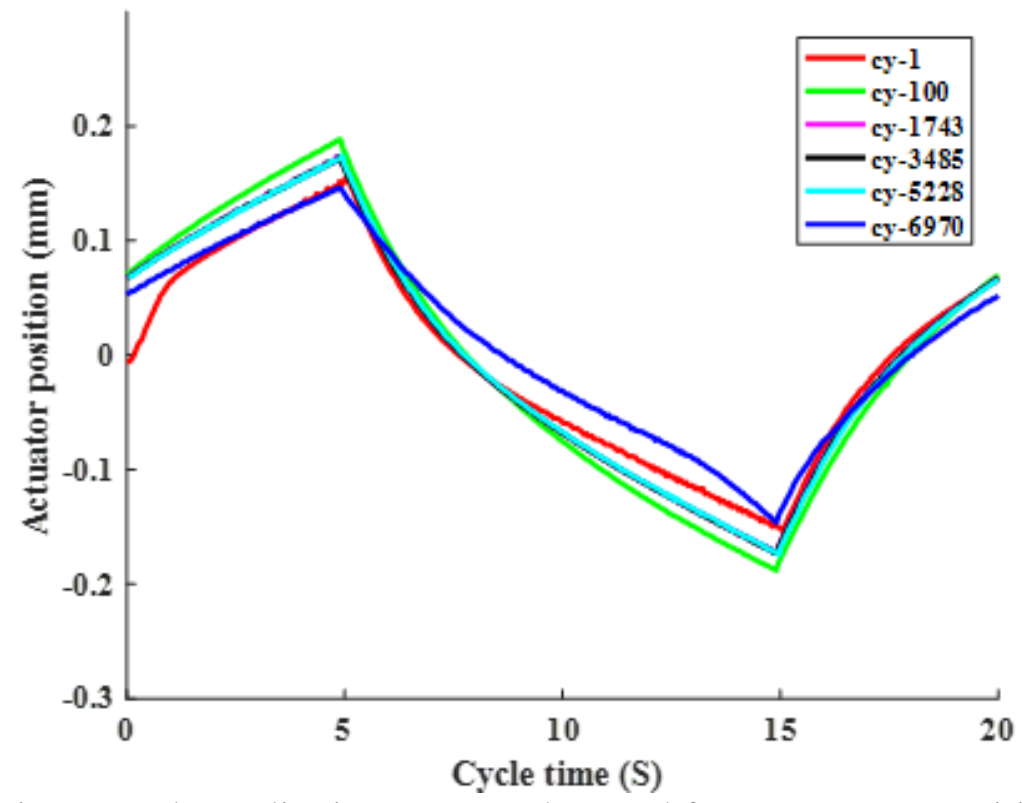

Figure 3C. 4 Intermittent cycle cyclic time versus observed frame actuator position (baseline removed) for ET-F41 fatigue test. 


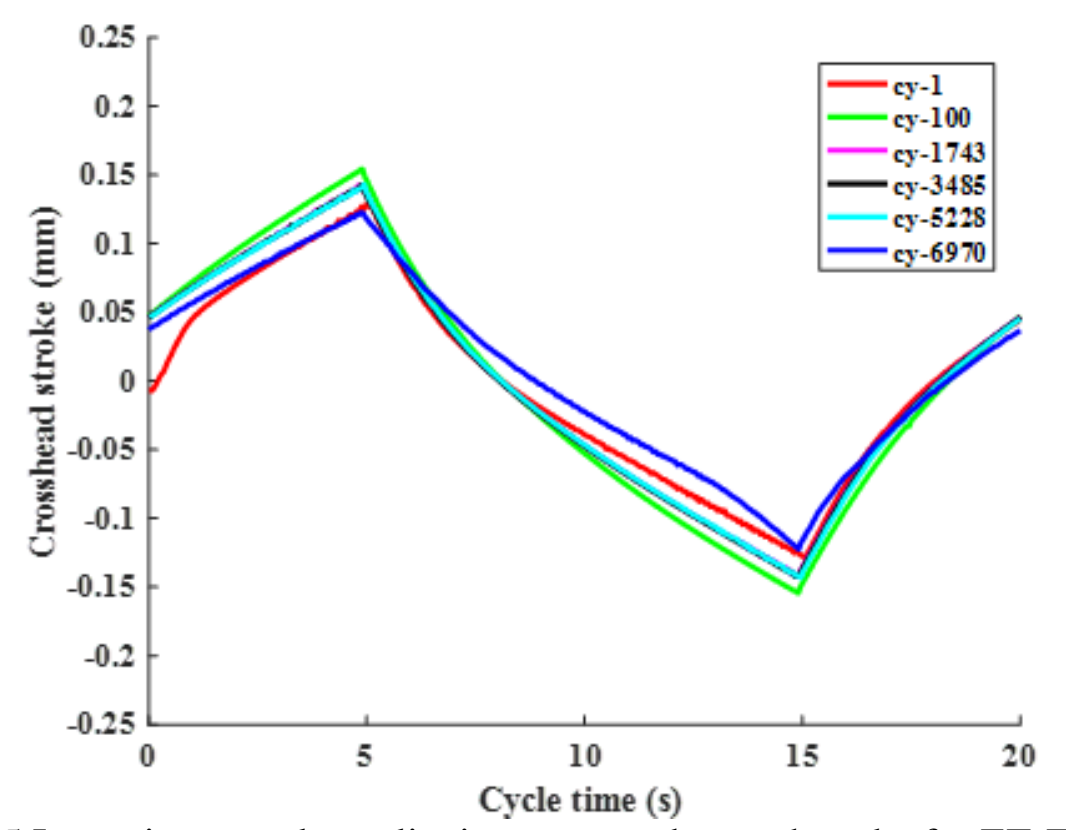

Figure 3C. 5 Intermittent cycle cyclic time versus observed stroke for ET-F41 fatigue test.

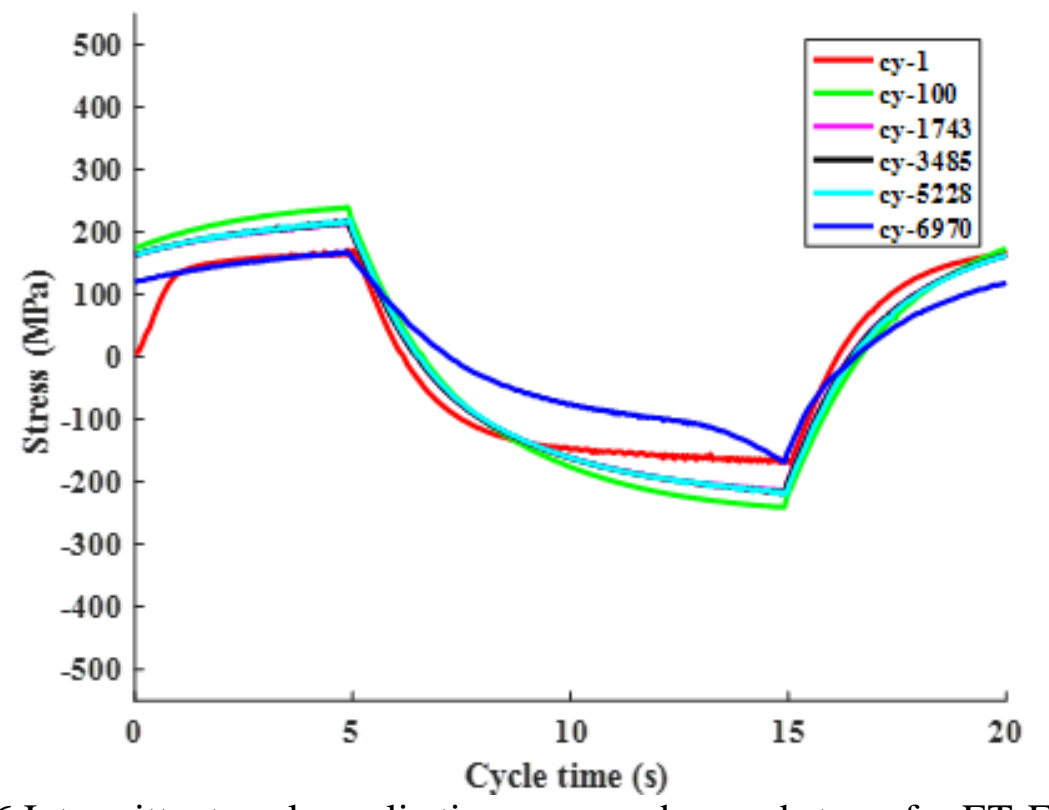

Figure 3C. 6 Intermittent cycle cyclic time versus observed stress for ET-F41 fatigue test. 


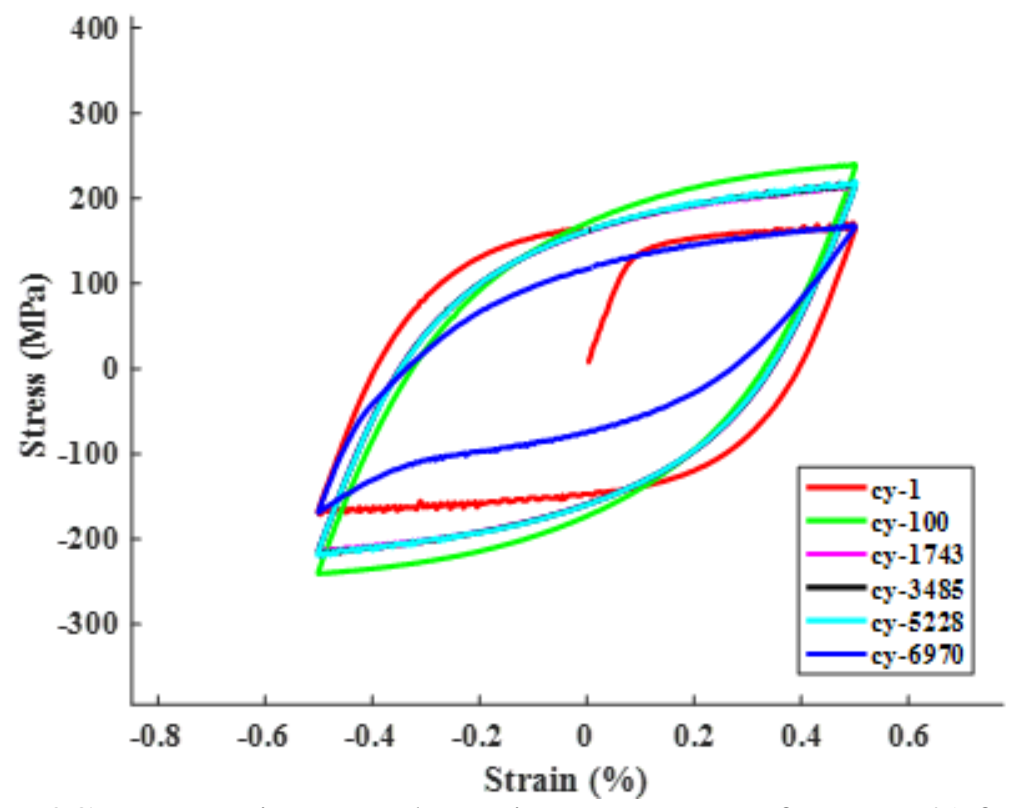

Figure 3C. 7 Intermittent cycle strain versus stress for ET-F41 fatigue test.

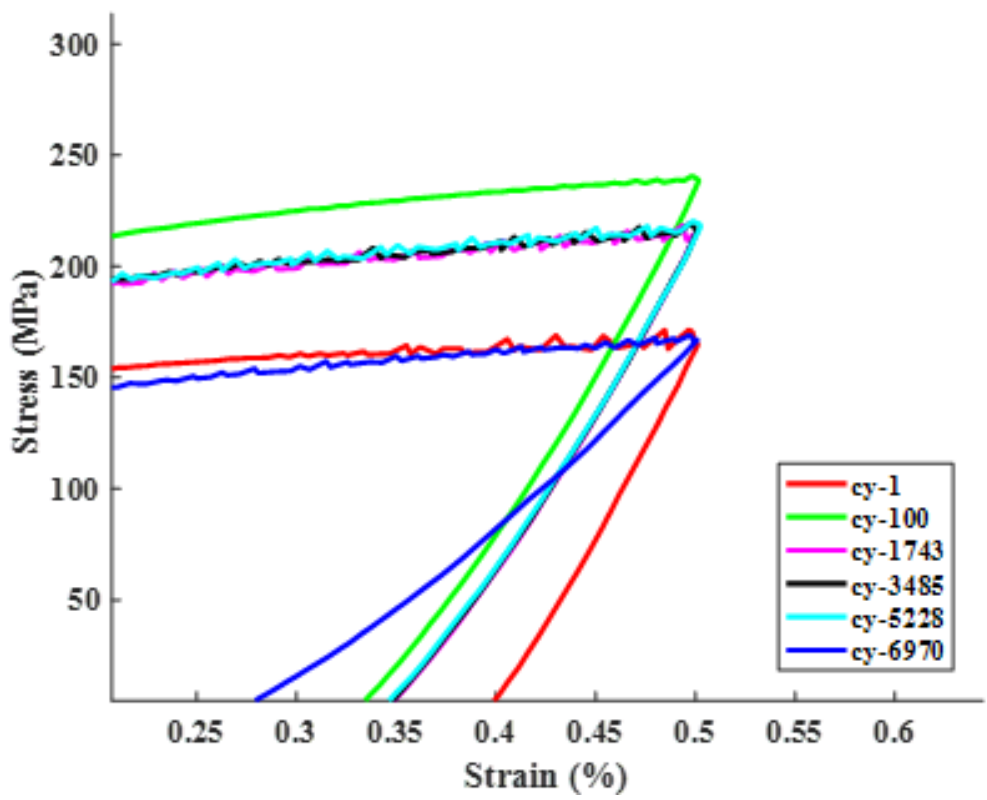

Figure 3C. 8 Magnified figure of Figure 3C. 8. 


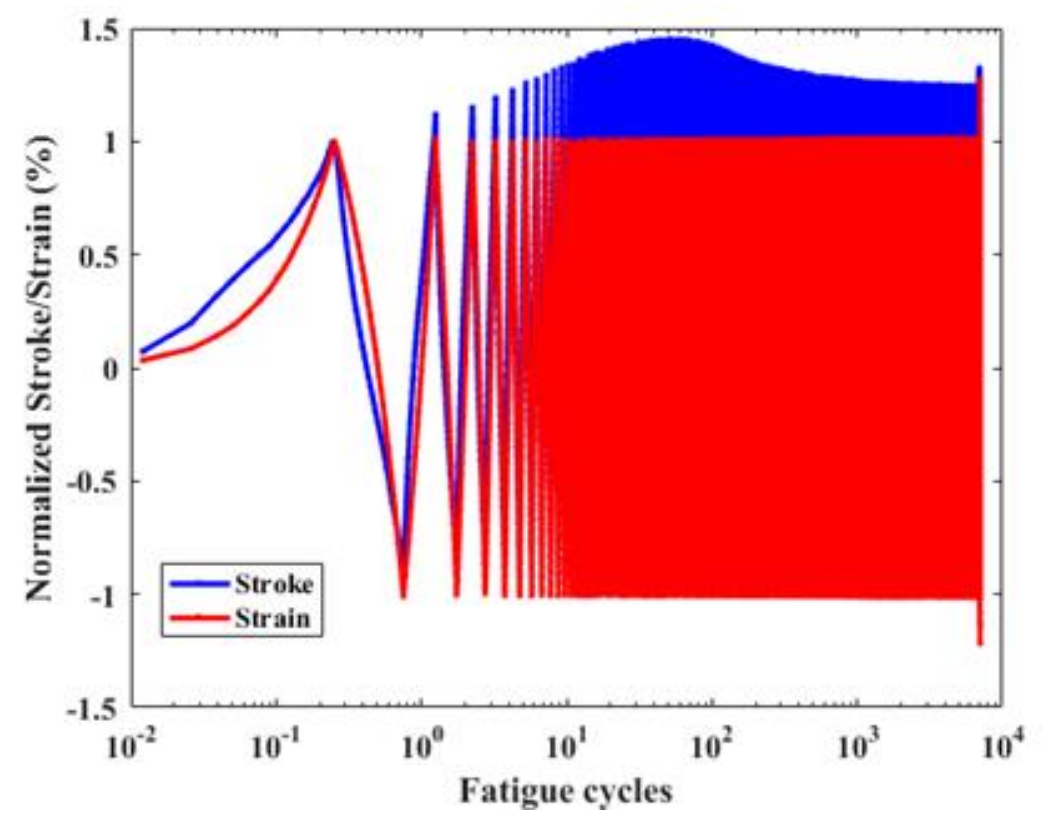

Figure 3C. 9 Time history of normalized stroke and strain measurements from ET-F41.

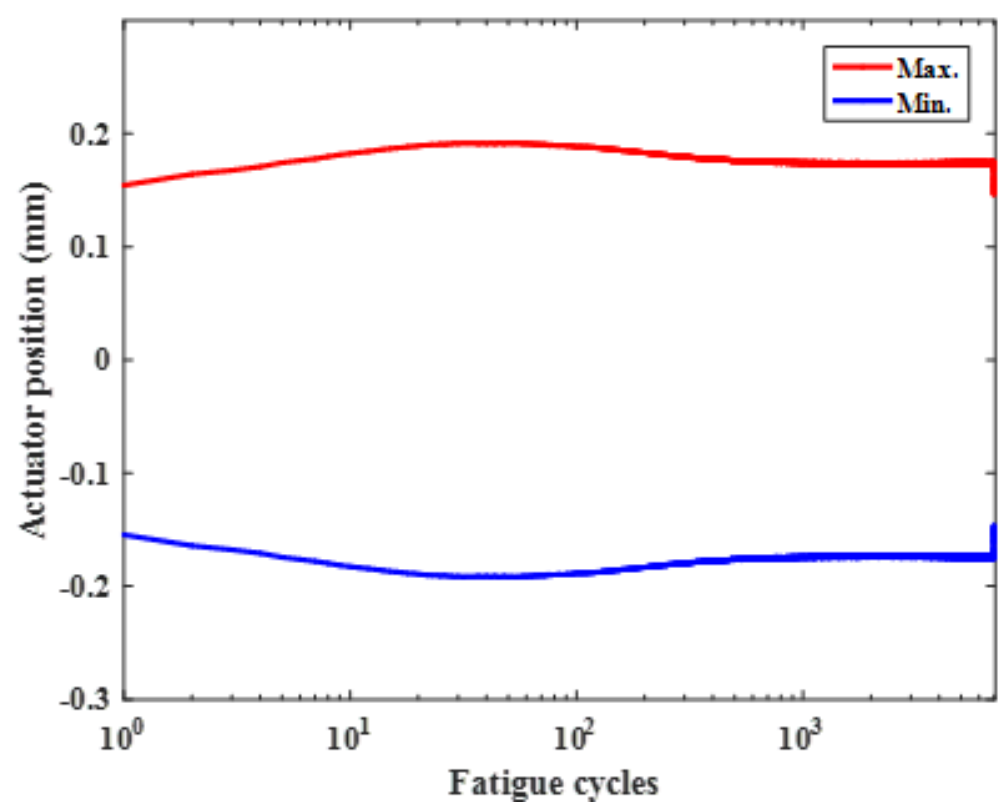

Figure 3C. 10 Maximum and minimum observed actuator position (baseline removed) during ET-F41. 


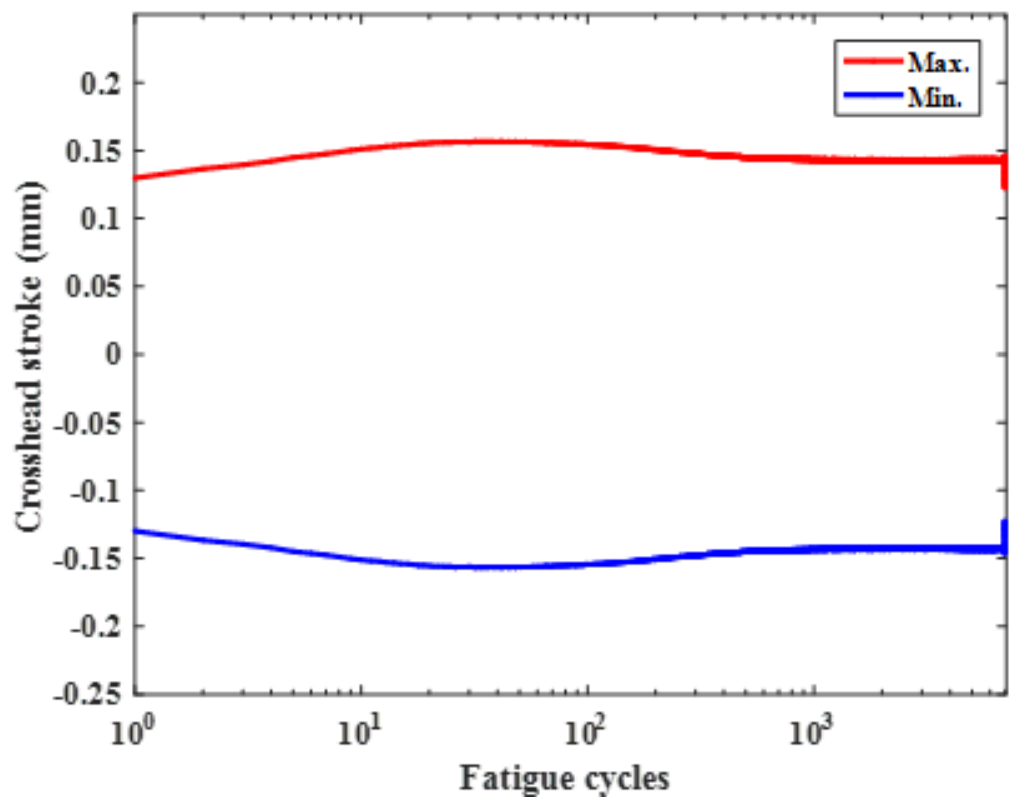

Figure 3C. 11 Maximum and minimum observed stroke (baseline removed) during ET-F41.

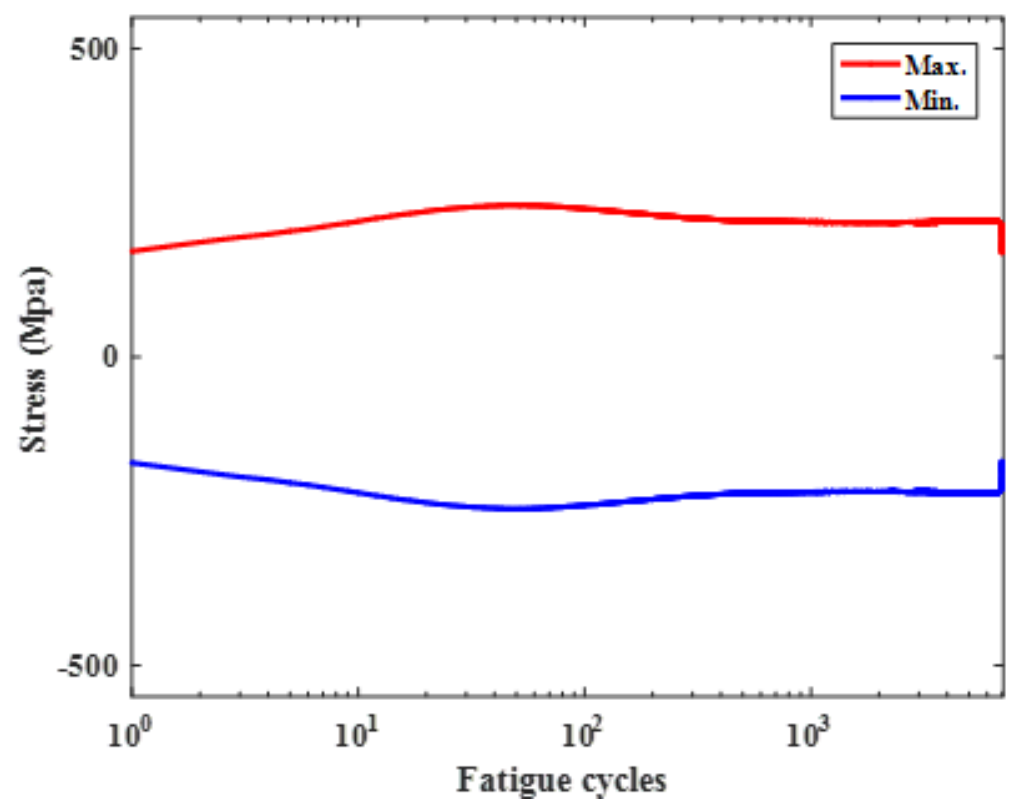

Figure 3C. 25 Maximum and minimum observed stress during ET-F41. 


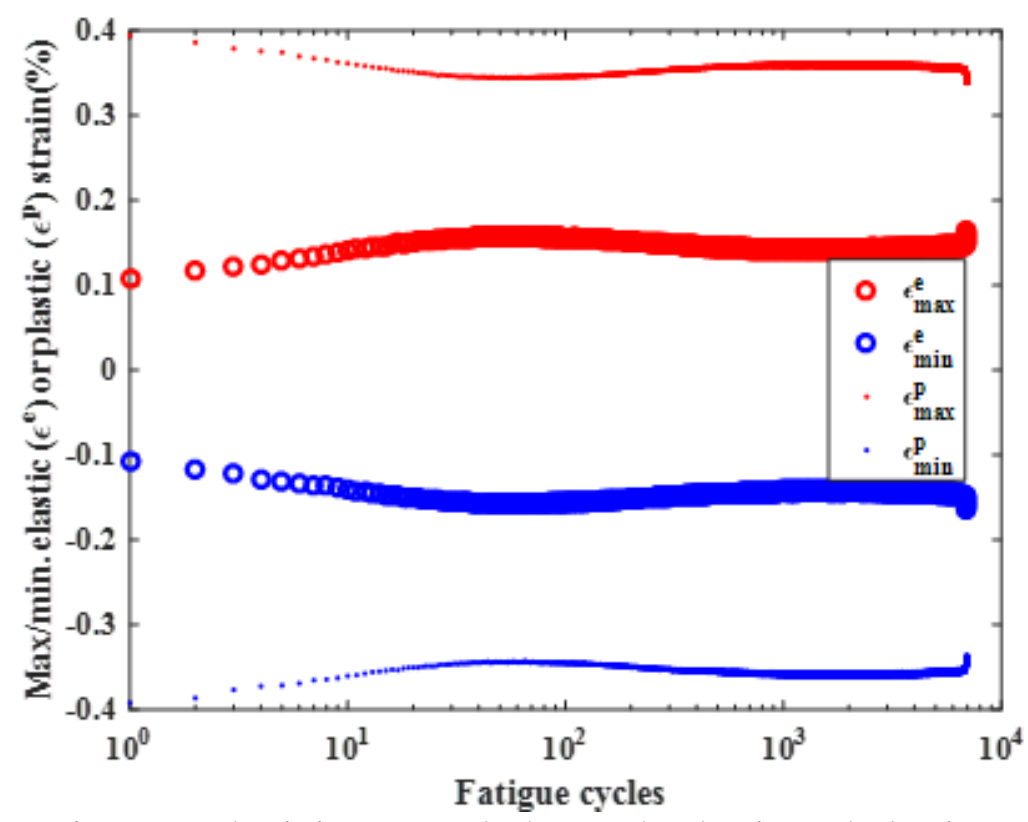

Figure 3C. 26 Maximum and minimum cycle-by-cycle elastic and plastic strain during ET-F41.

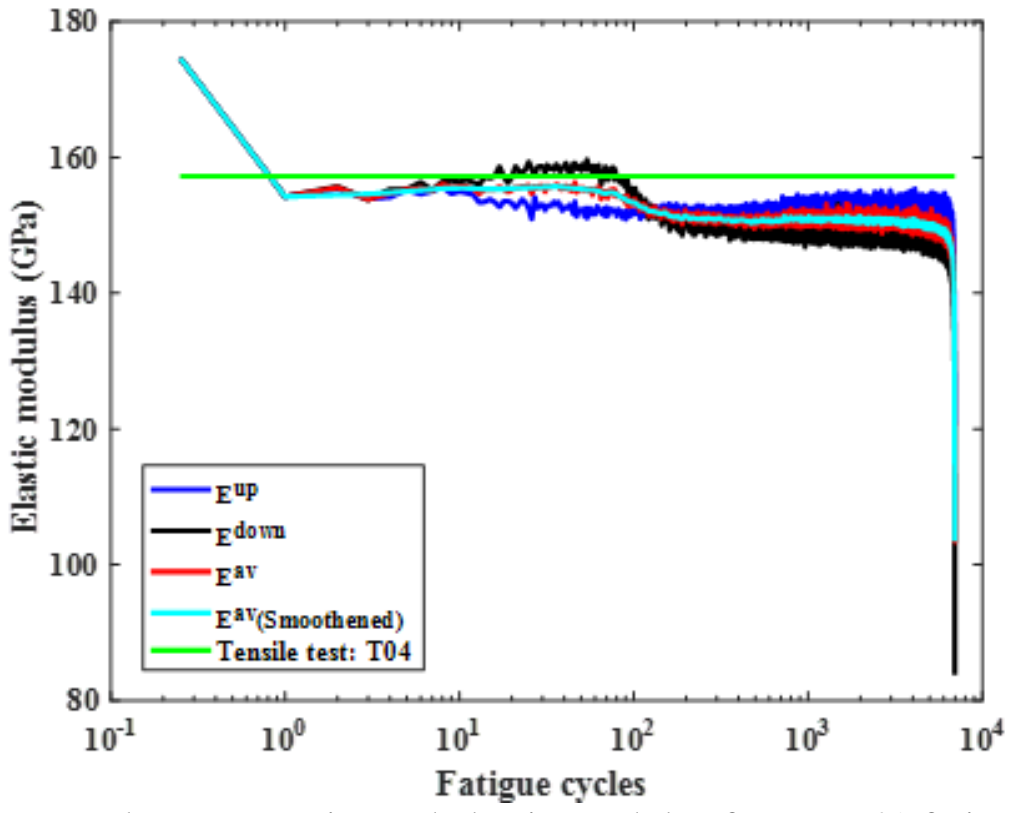

Figure 3C. 27 Fatigue cycle versus estimated elastic modulus for ET-F41 fatigue test. Also the figure shows the comparison with respect to corresponding tensile test (ET-T04) elastic modulus. 


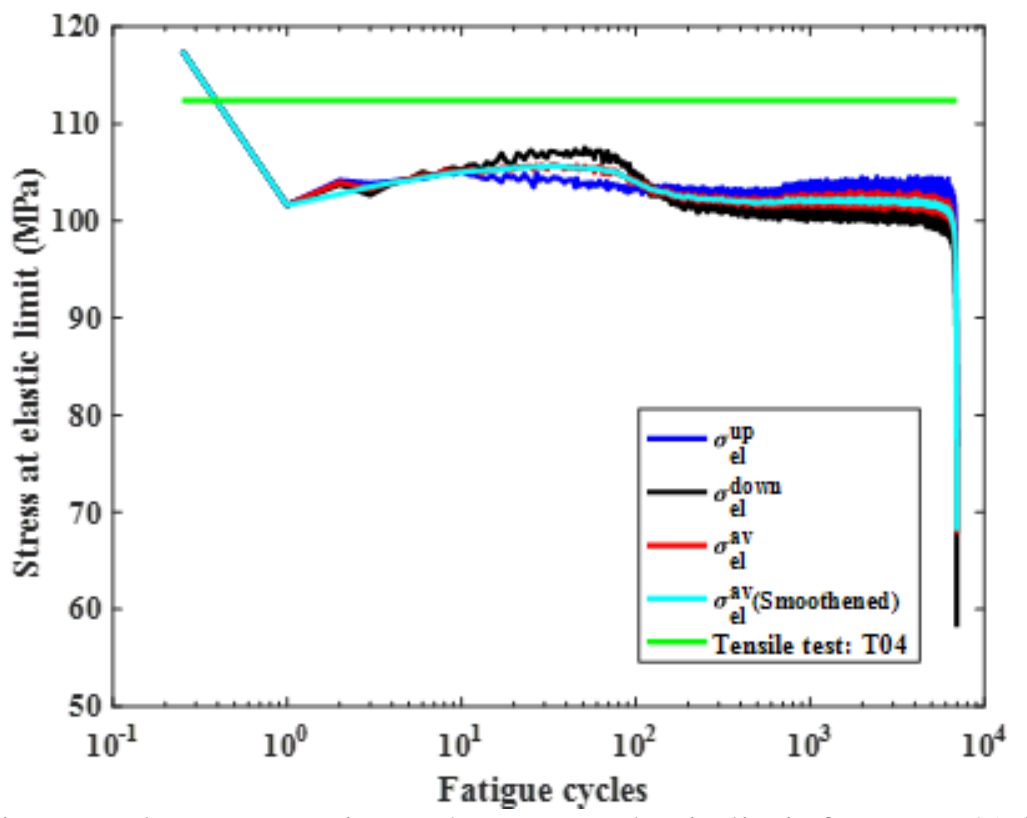

Figure 3C. 28 Fatigue cycle versus estimated stress at elastic limit for ET-F41 fatigue test. Also the figure shows the comparison with respect to corresponding tensile test (ET-T04) elastic limit stress.

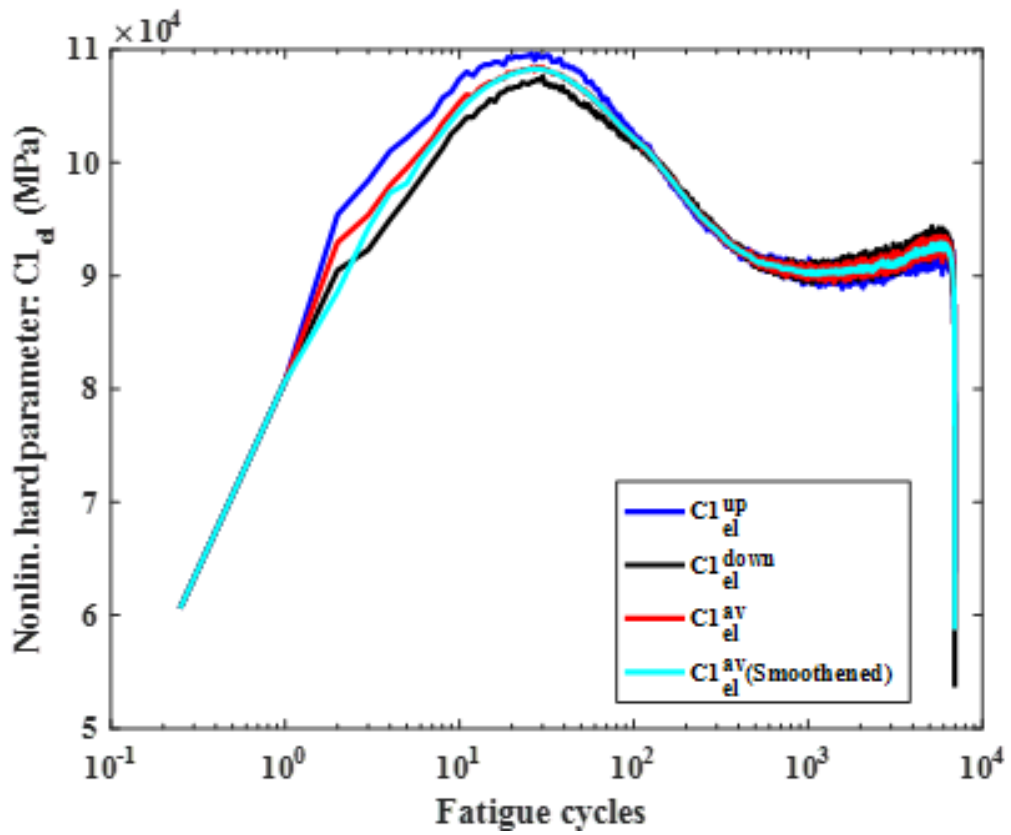

Figure 3C. 29 Fatigue cycle versus estimated nonlinear kinematic hardening or Chaboche model parameters $\left(\mathrm{C} 1_{\mathrm{el}}\right)$ for ET-F41 fatigue test, with cyclic elastic limit stress was considered to estimate the corresponding cycle back stress. 


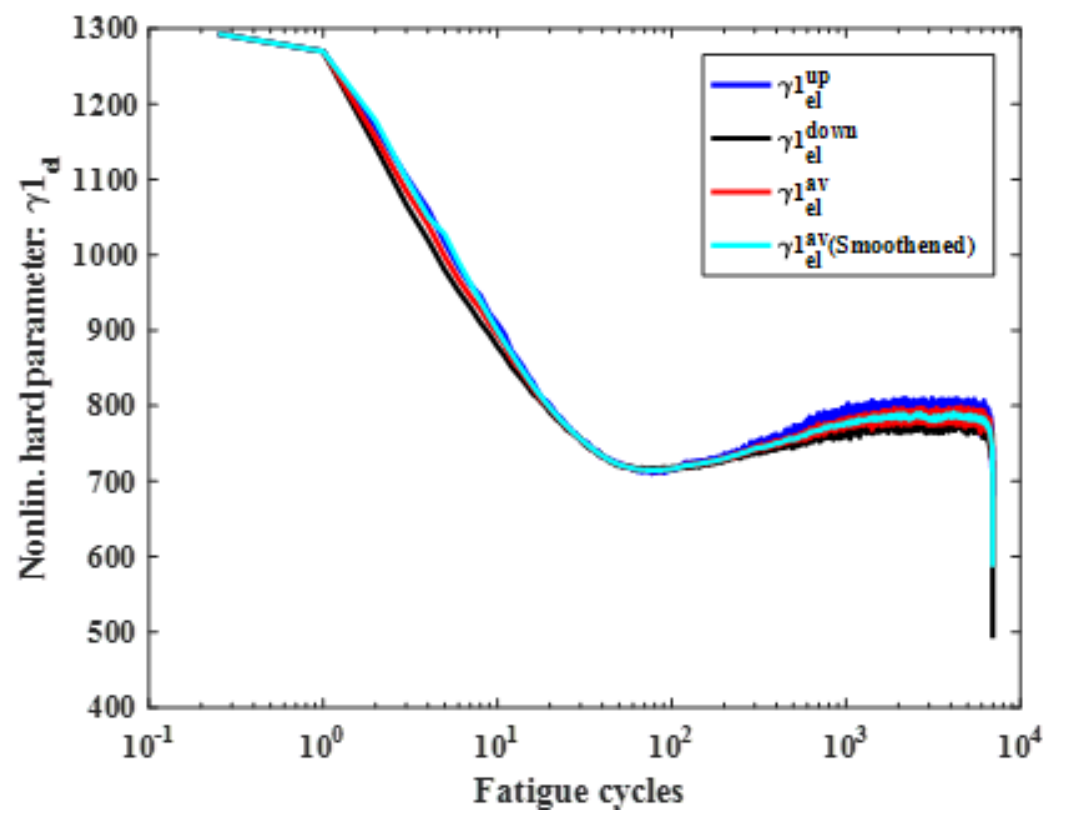

Figure 3C. 30 Fatigue cycle versus estimated nonlinear kinematic hardening or Chaboche model parameters $\left(\gamma 1_{\mathrm{el}}\right)$ for ET-F41 fatigue test, with cyclic elastic limit stress was considered to estimate the corresponding cycle back stress.

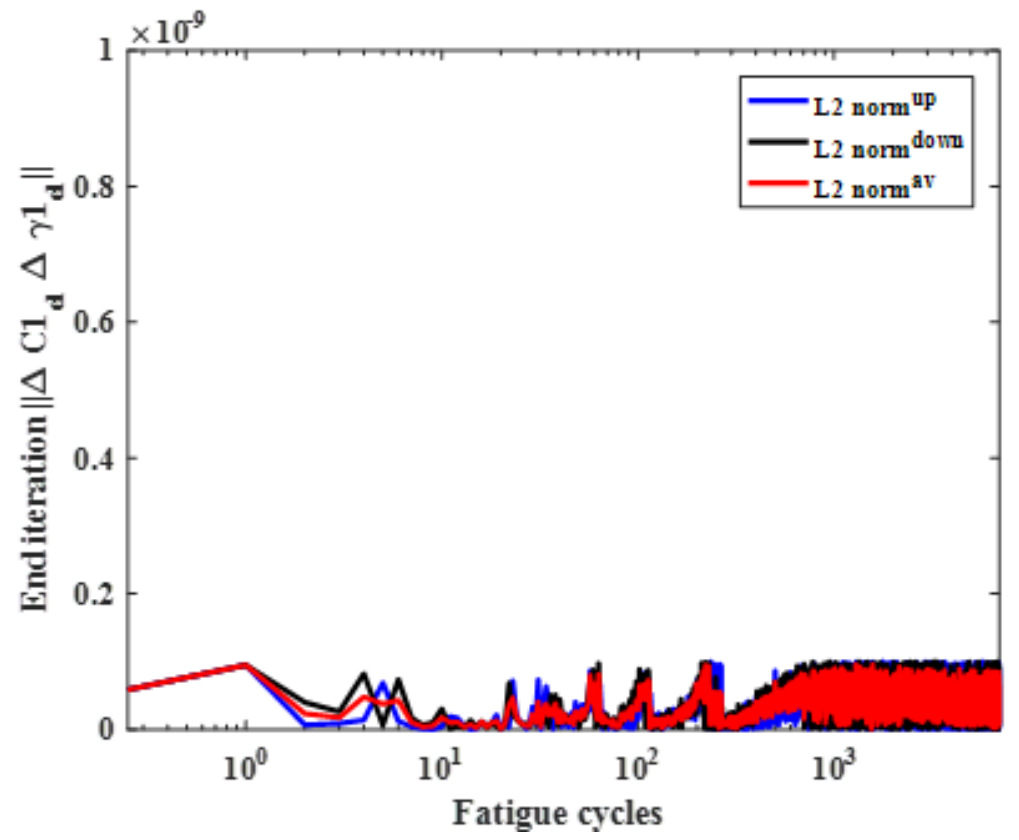

Figure 3C. 31 Fatigue cycle versus end iteration $\mathrm{L}_{2}$ norm of the incremental nonlinear Chaboche model parameters $\left(\mathrm{C} 1_{\mathrm{el}}\right.$ and $\left.\gamma 1_{\mathrm{el}}\right)$ for ET-F06 fatigue test, with cyclic elastic limit stress was considered to estimate the corresponding cycle back stress. 


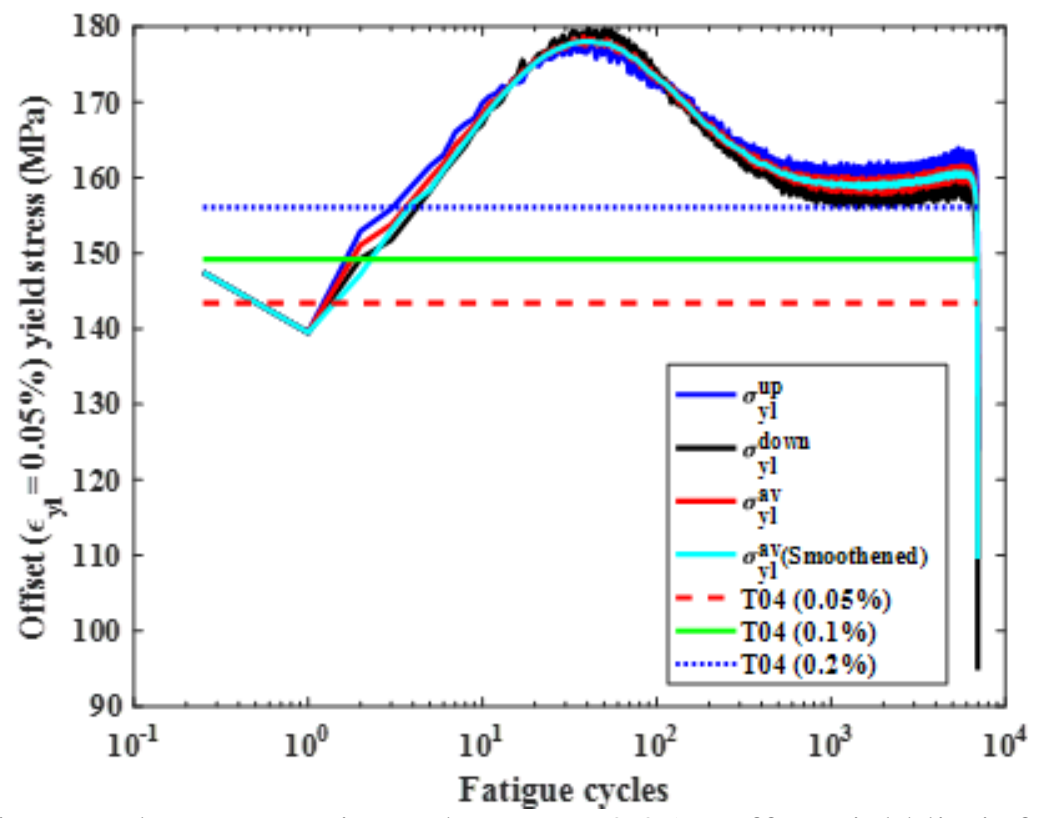

Figure 3C. 32 Fatigue cycle versus estimated stress at $0.05 \%$ offset yield limit for ET-F41 fatigue test. Also the figure shows the comparison with respect to corresponding tensile test (ET-T04) yield limit stresses.

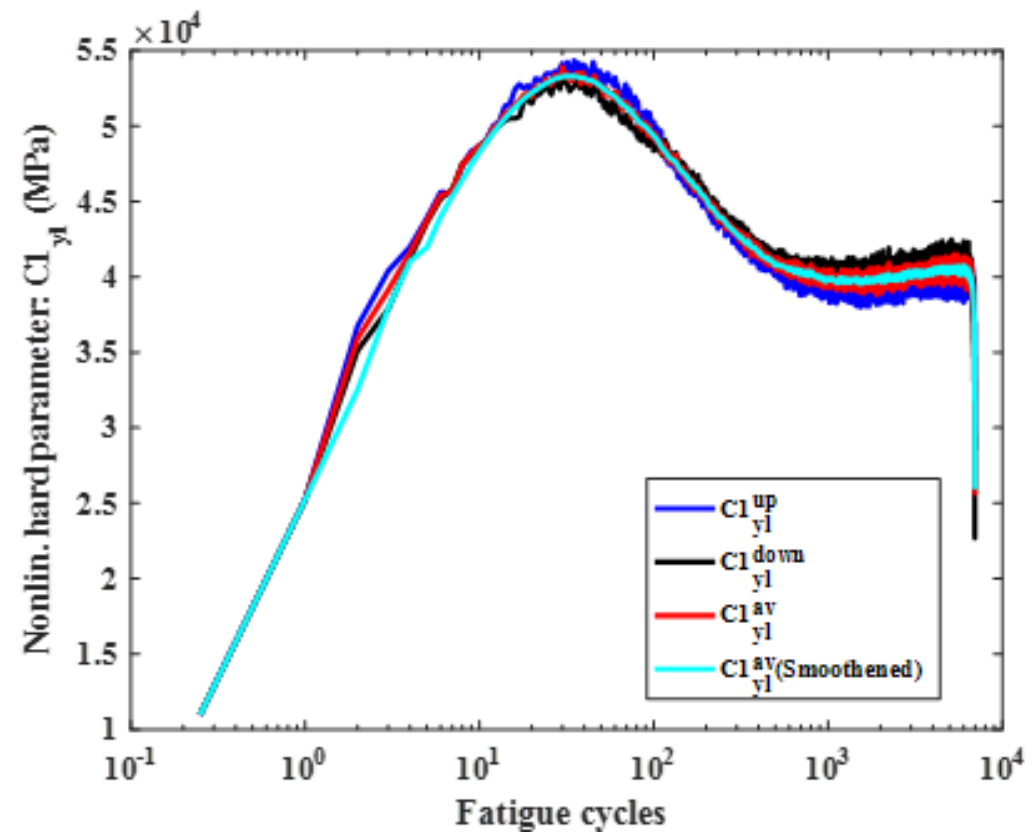

Figure 3C. 33 Fatigue cycle versus estimated nonlinear kinematic hardening or Chaboche model parameter $\left(\mathrm{C}_{\mathrm{yl}}\right)$ for ET-F41 fatigue test, with cyclic $0.05 \%$ offset yield limit stress was considered to estimate the corresponding cycle back stress. 


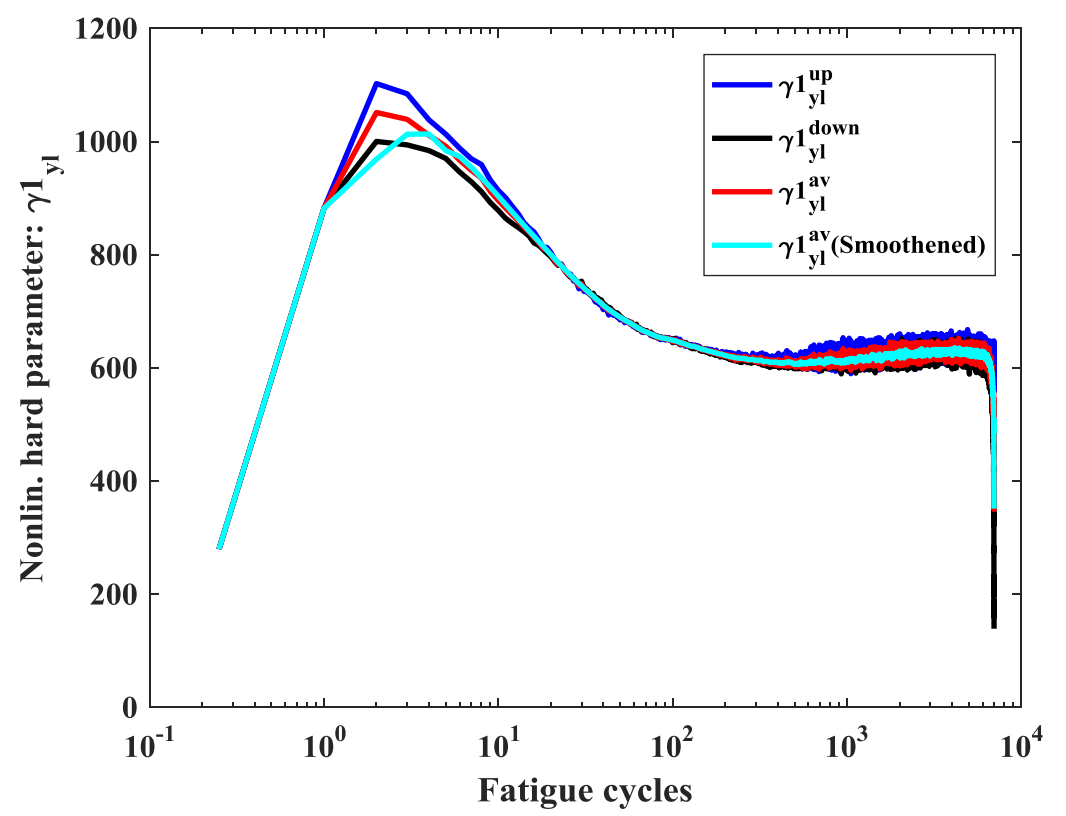

Figure 3C. 34 Fatigue cycle versus estimated nonlinear kinematic hardening or Chaboche model parameter $\left(\gamma 1_{\mathrm{yl}}\right)$ for ET-F41 fatigue test, with cyclic $0.05 \%$ offset yield limit stress was considered to estimate the corresponding cycle back stress.

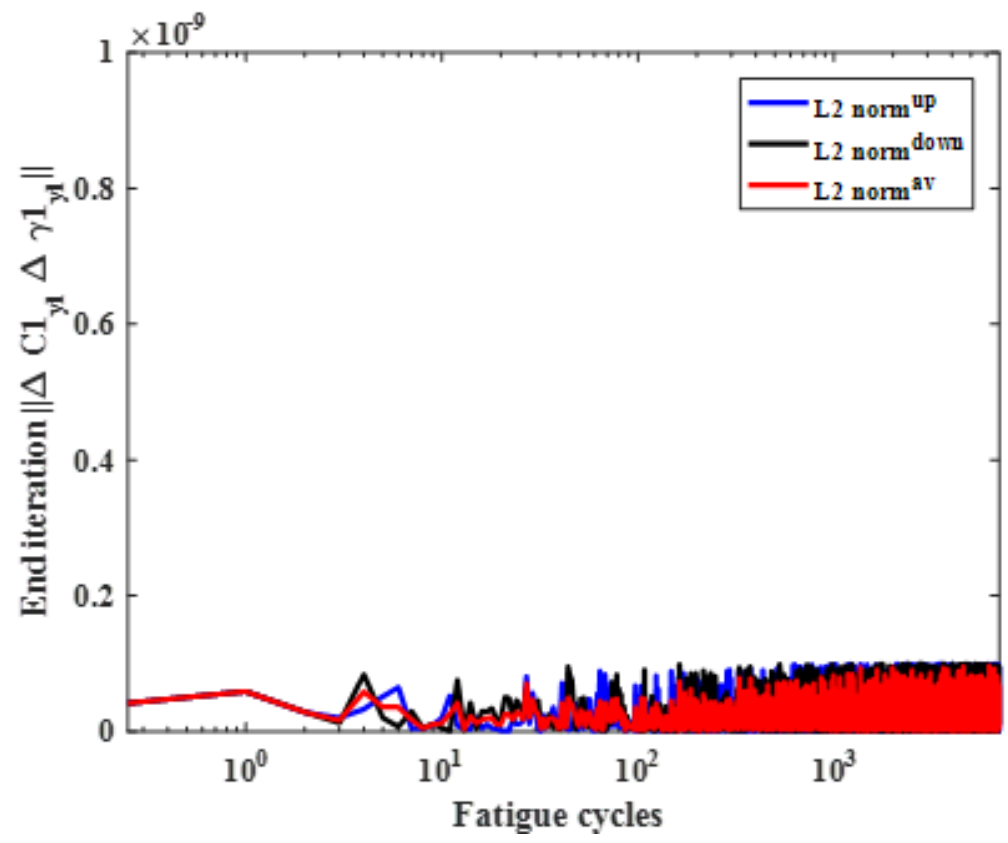

Figure 3C. 35 Fatigue cycle versus end iteration $\mathrm{L}_{2}$ norm of the incremental nonlinear Chaboche model parameters $\left(\mathrm{C}_{\mathrm{yl}}, \gamma 1_{\mathrm{yl}}\right)$ for ET-F41 fatigue test, with cyclic $0.05 \%$ offset yield limit stress was considered to estimate the corresponding cycle back stress. 


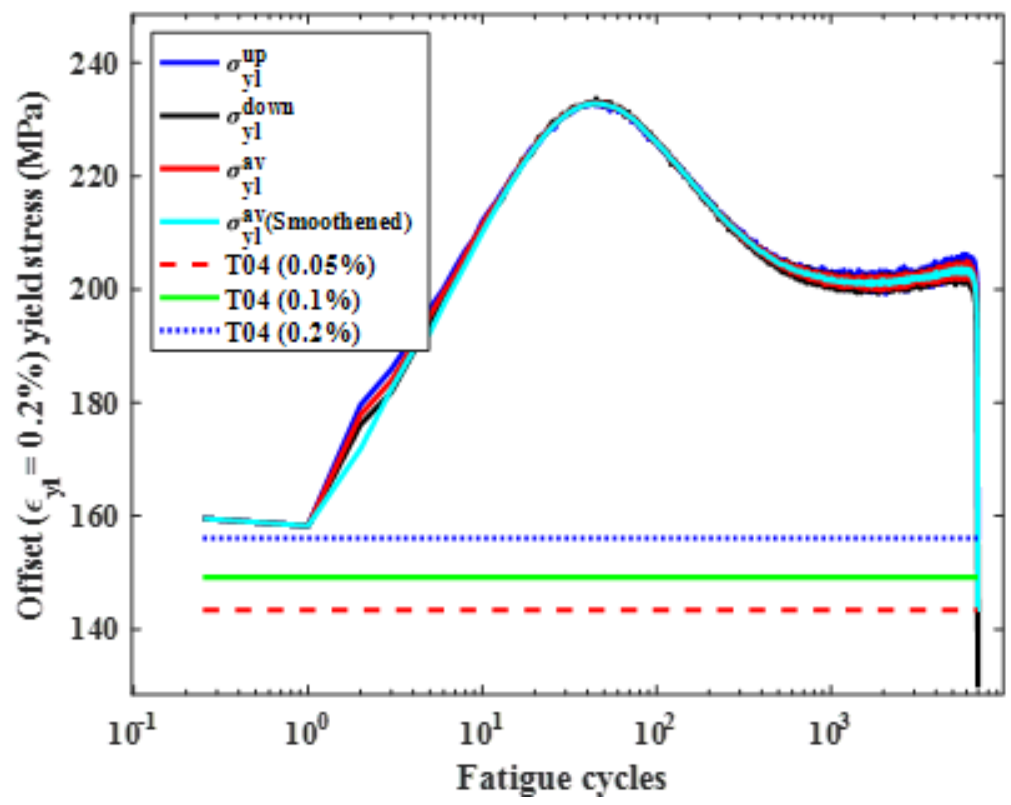

Figure 3C. 36 Fatigue cycle versus estimated stress at $0.2 \%$ offset yield limit for ET-F41 fatigue test. Also the figure shows the comparison with respect to corresponding tensile test (ET-T04) yield limit stresses.

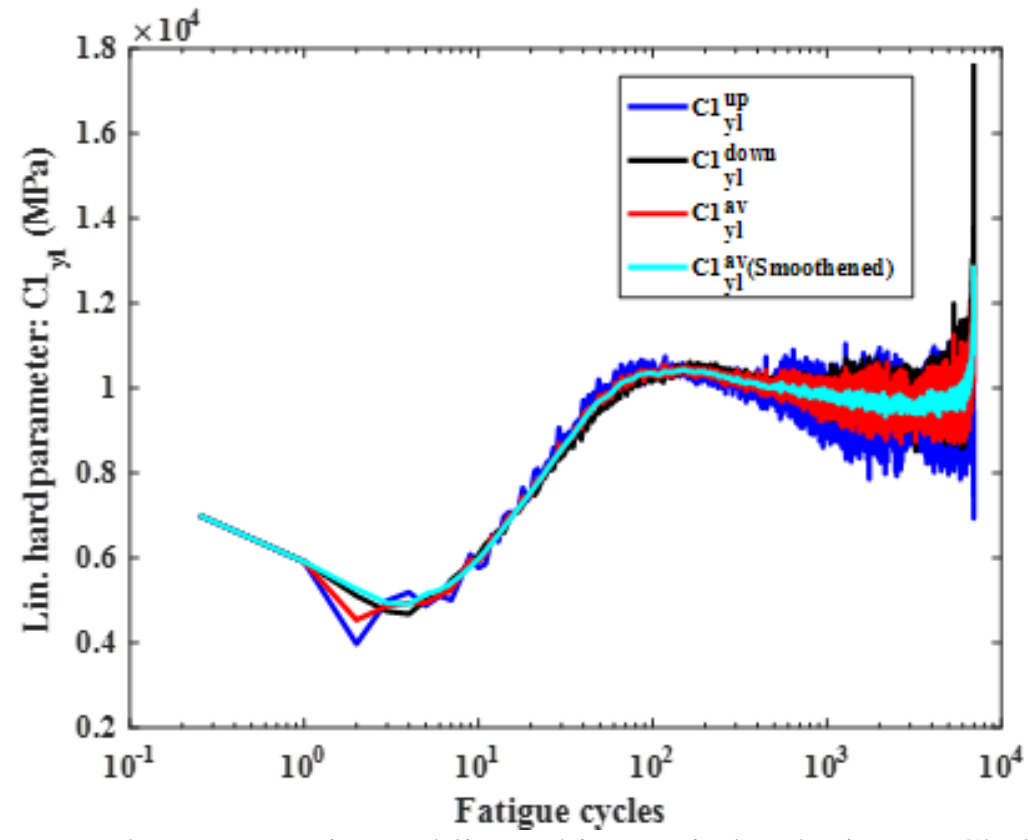

Figure 3C. 37 Fatigue cycle versus estimated linear kinematic hardening or Chaboche model parameter $\left(\mathrm{C} 1_{\mathrm{yl}}\right)$ for ET-F41 fatigue test, with cyclic $0.2 \%$ offset yield limit stress was considered to estimate the corresponding cycle back stress. 


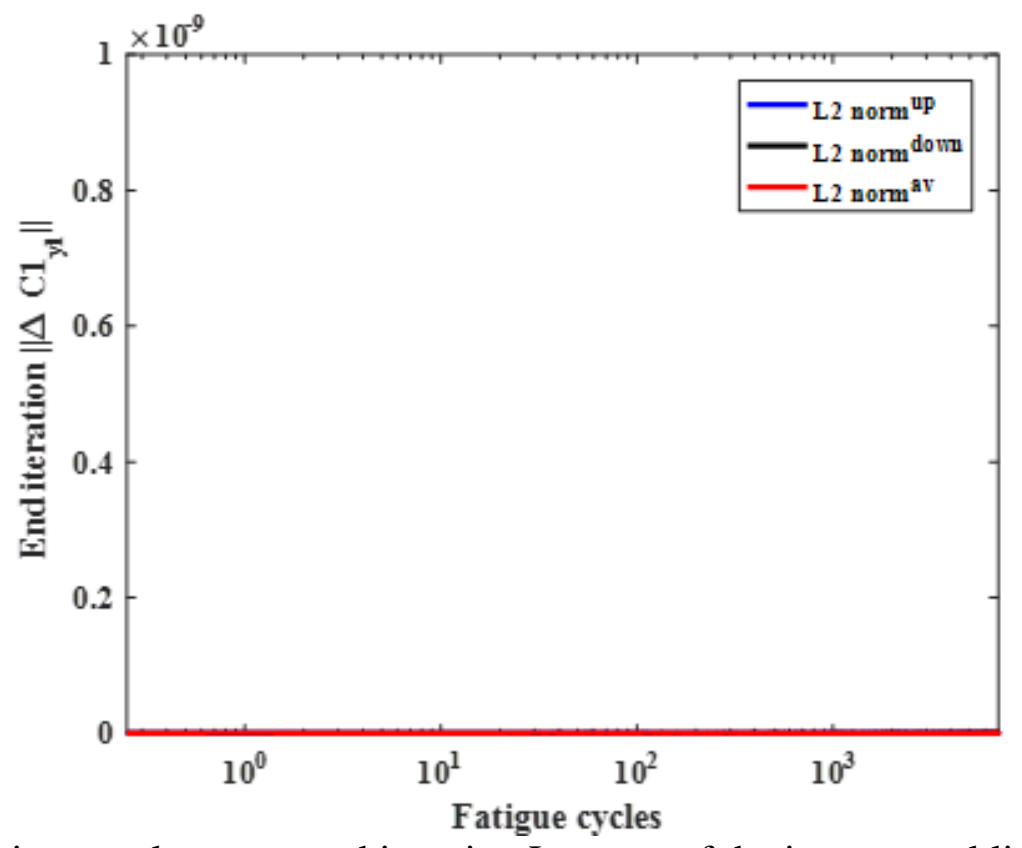

Figure 3C. 38 Fatigue cycle versus end iteration $\mathrm{L}_{2}$ norm of the incremental linear Chaboche model parameters $\left(\mathrm{C}_{\mathrm{yl}}\right)$ for ET-F41 fatigue test, with cyclic $0.2 \%$ offset yield limit stress was considered to estimate the corresponding cycle back stress.

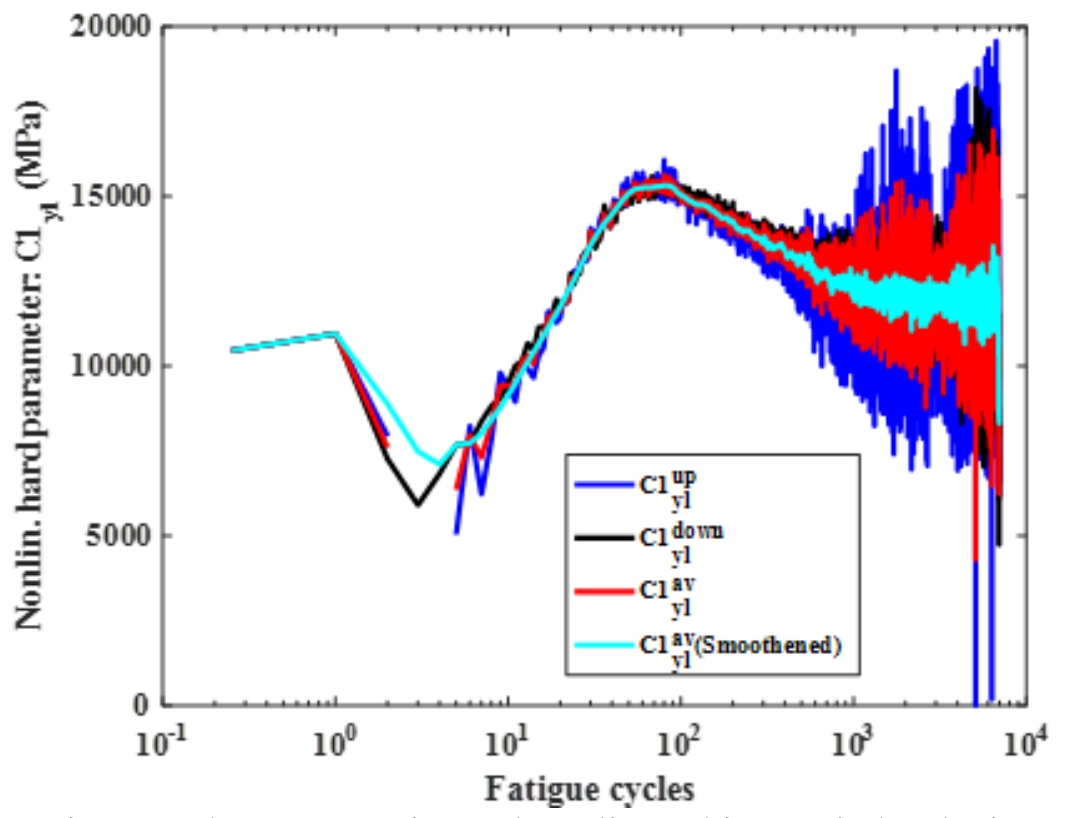

Figure 3C. 39 Fatigue cycle versus estimated nonlinear kinematic hardening or Chaboche model parameter $\left(\mathrm{Cl}_{\mathrm{yl}}\right)$ for ET-F41 fatigue test, with cyclic $0.2 \%$ offset yield limit stress was considered to estimate the corresponding cycle back stress. 


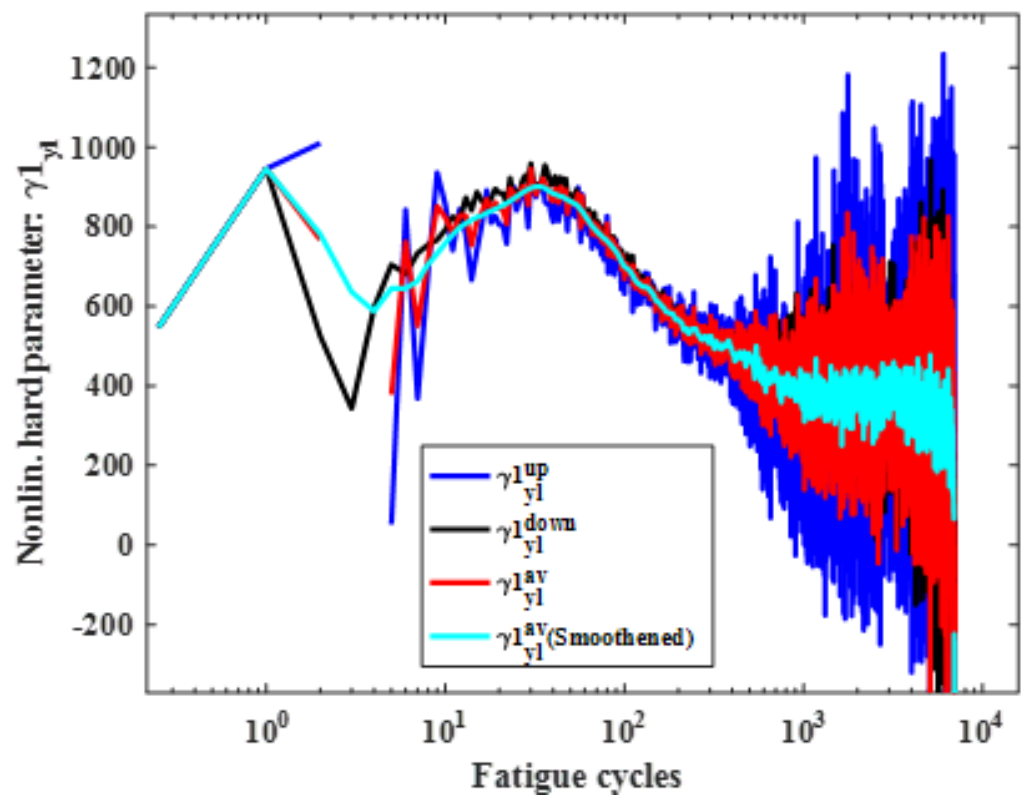

Figure 3C. 40 Fatigue cycle versus estimated nonlinear kinematic hardening or Chaboche model parameter $\left(\gamma 1_{\mathrm{yl}}\right)$ for ET-F41 fatigue test, with cyclic $0.2 \%$ offset yield limit stress was considered to estimate the corresponding cycle back stress.

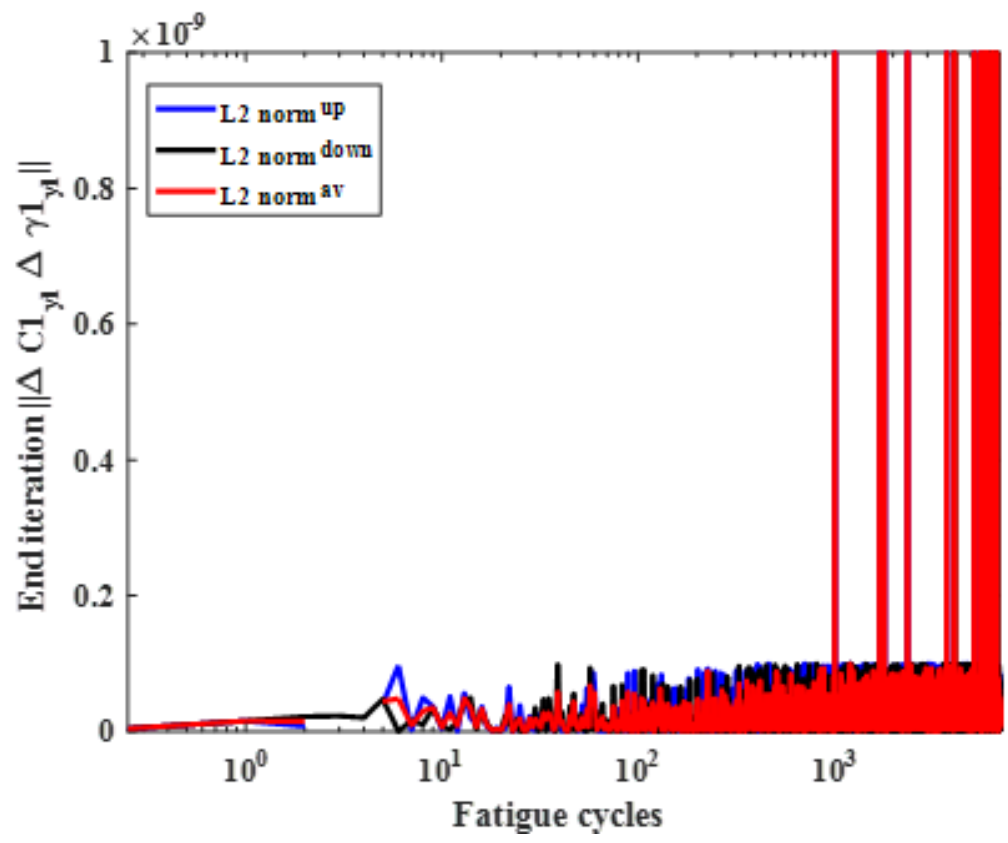

Figure 3C. 41 Fatigue cycle versus end iteration $\mathrm{L}_{2}$ norm of the incremental nonlinear Chaboche model parameters $\left(\mathrm{C}_{\mathrm{yl}}, \gamma 1_{\mathrm{yl}}\right)$ for ET-F41 fatigue test, with cyclic $0.2 \%$ offset yield limit stress was considered to estimate the corresponding cycle back stress. Note: high values for iteration norms and, therefore, nonlinear kinematic hardening parameters $\left(\mathrm{Cl}_{\mathrm{yl}}, \gamma 1_{\mathrm{yl}}\right)$ based on $0.2 \%$ offset yield stress should not be used for modeling fatigue behavior of $316 \mathrm{SS}$. 


\section{Appendix-3D: Supplementary Results for ET-F38 Fatigue Test}

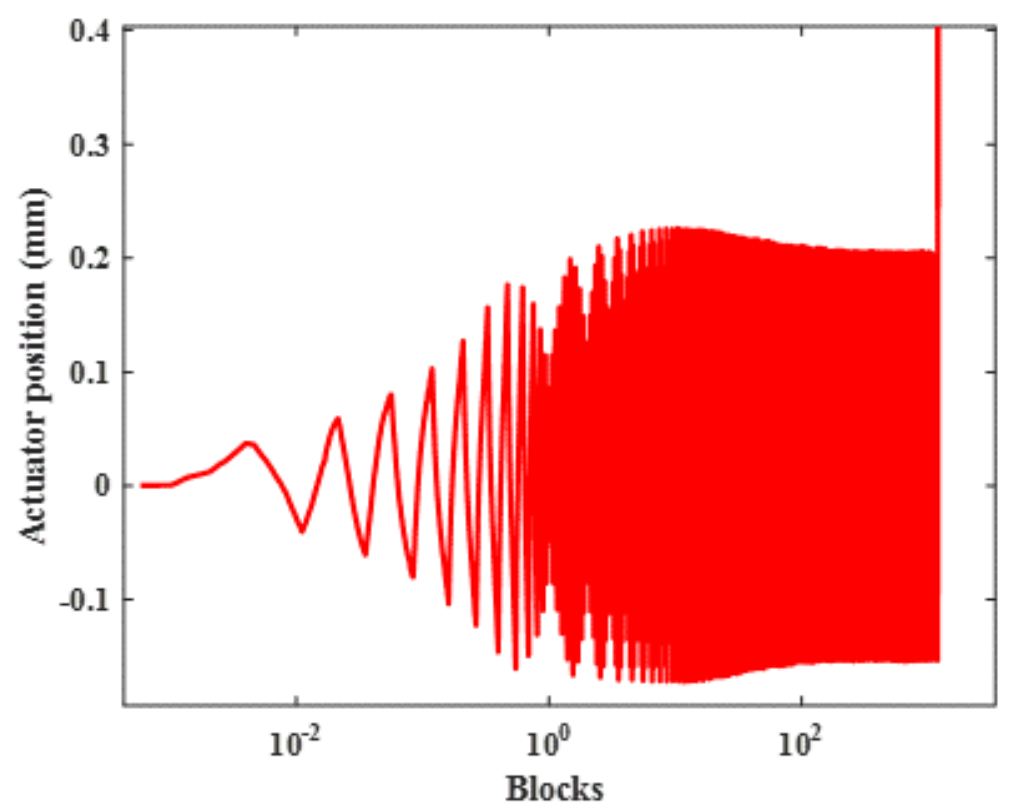

Figure 3D. 1 Observed actuator position during ET-F38.

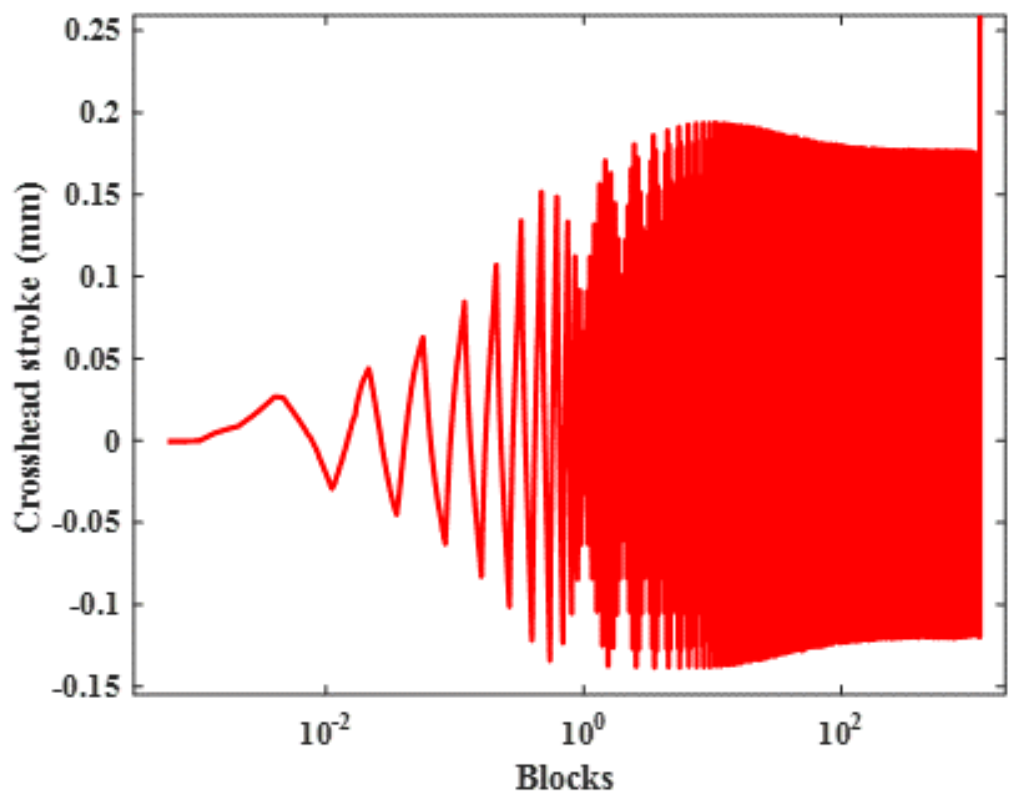

Figure 3D. 2 Observed stroke during ET-F38. 


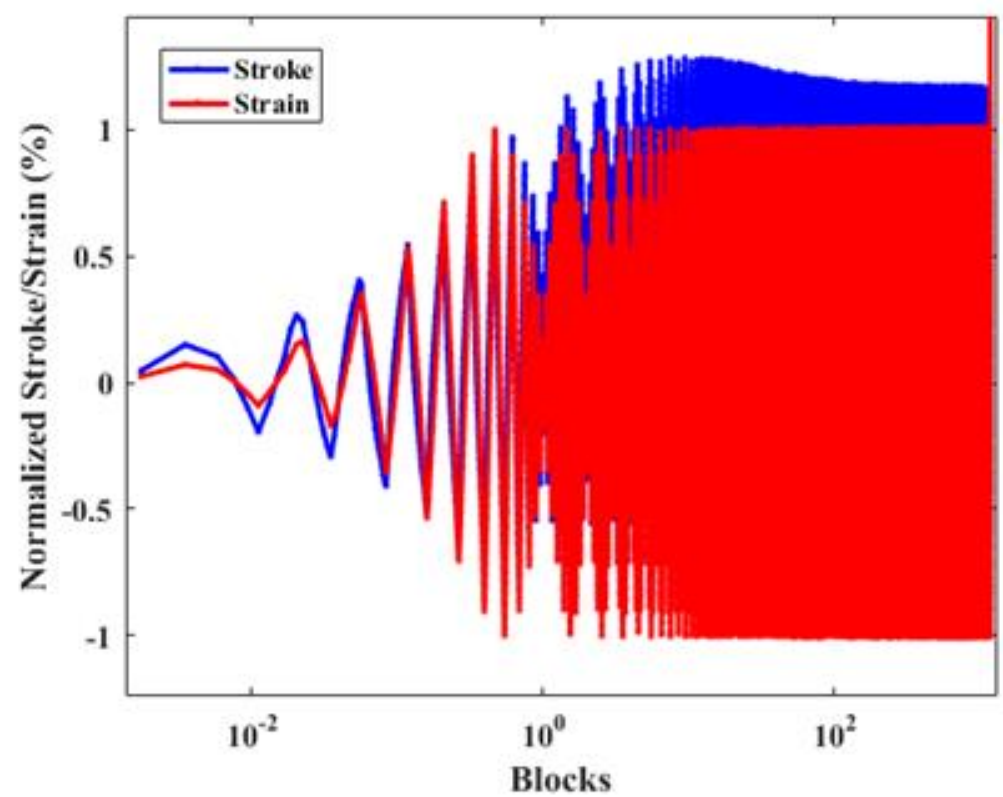

Figure 3D. 3 Time history of normalized stroke and strain measurements from ET-F38.

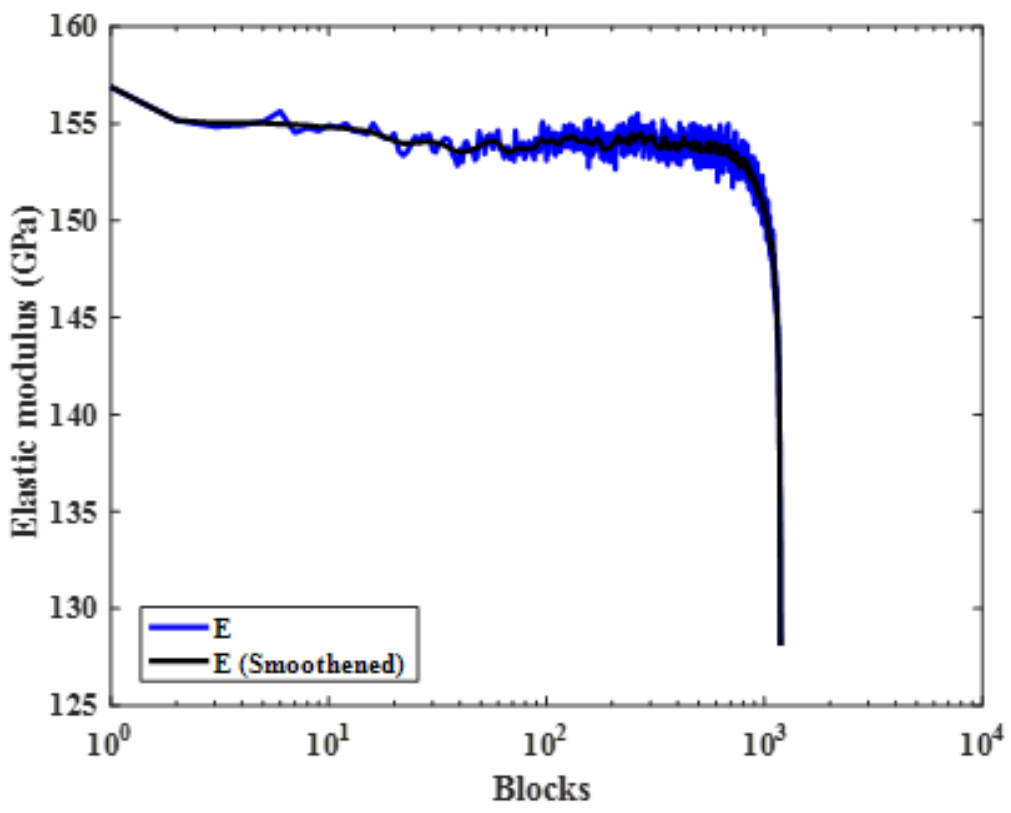

Figure 3D. 4 Block-by-block estimated elastic modulus for ET-F38 fatigue test. 


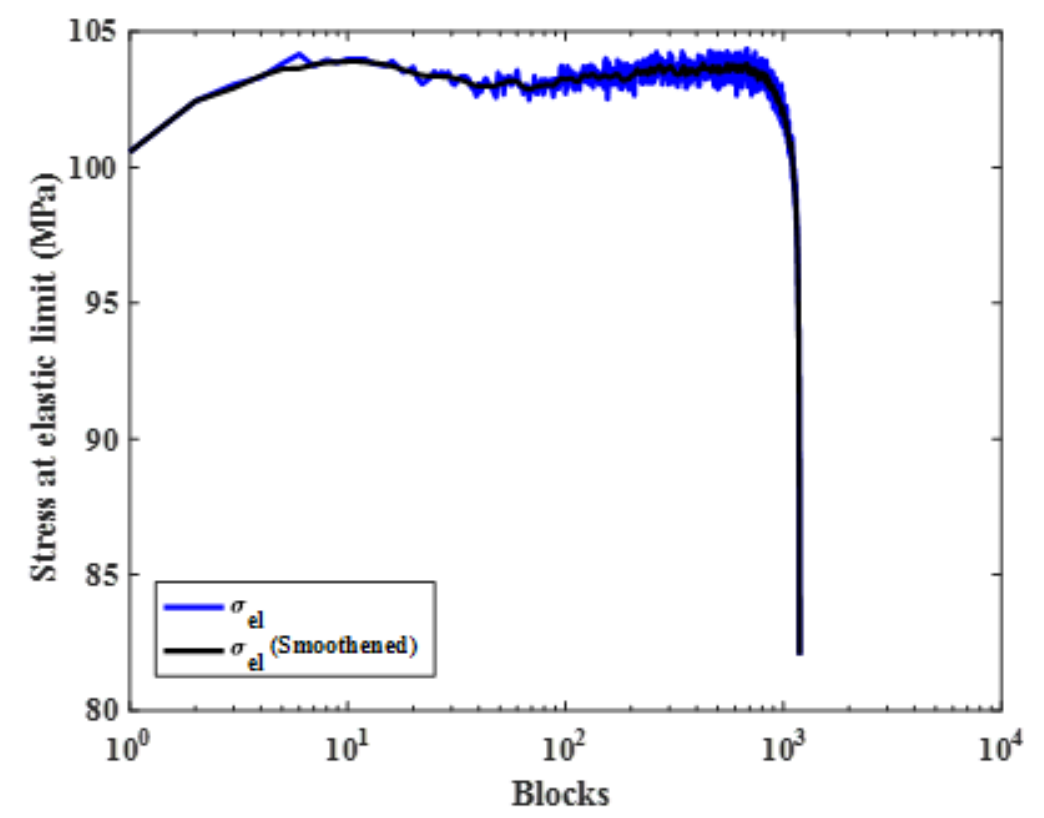

Figure 3D. 5 Block-by-block estimated stress at elastic limit for ET-F38 fatigue test.

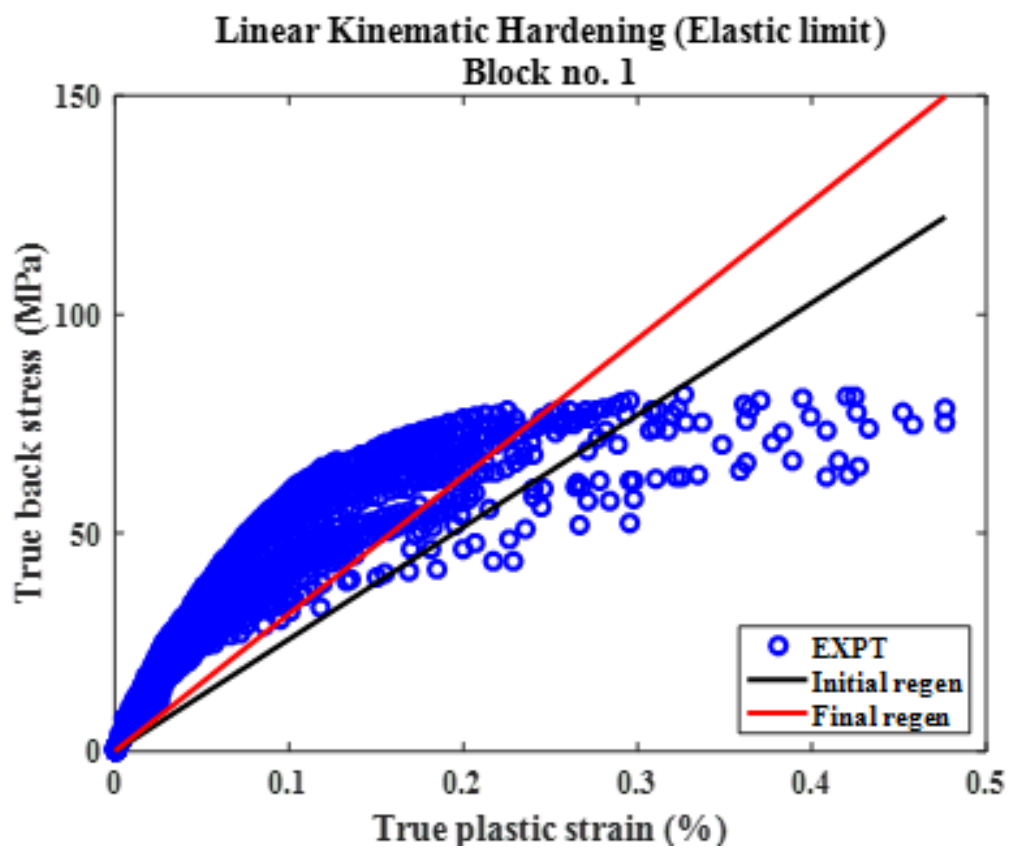

Figure 3D. 6 Comparison of experimental true back stress (as a function of true plastic strain) and predicted back stress (using linear kinematic hardening equation and estimated parameter $\mathrm{C} 1$, considering elastic limit stress as yield stress) for block-1. Solid line: prediction; Circles: experimental

data from 12 fatigue cycles of block-1. Note: Bad prediction and, therefore, block-by-block linear kinematic hardening parameter $\mathrm{C} 1_{\mathrm{el}}$ based on elastic limit stress should not be used for modeling fatigue behavior of $316 \mathrm{SS}$. 


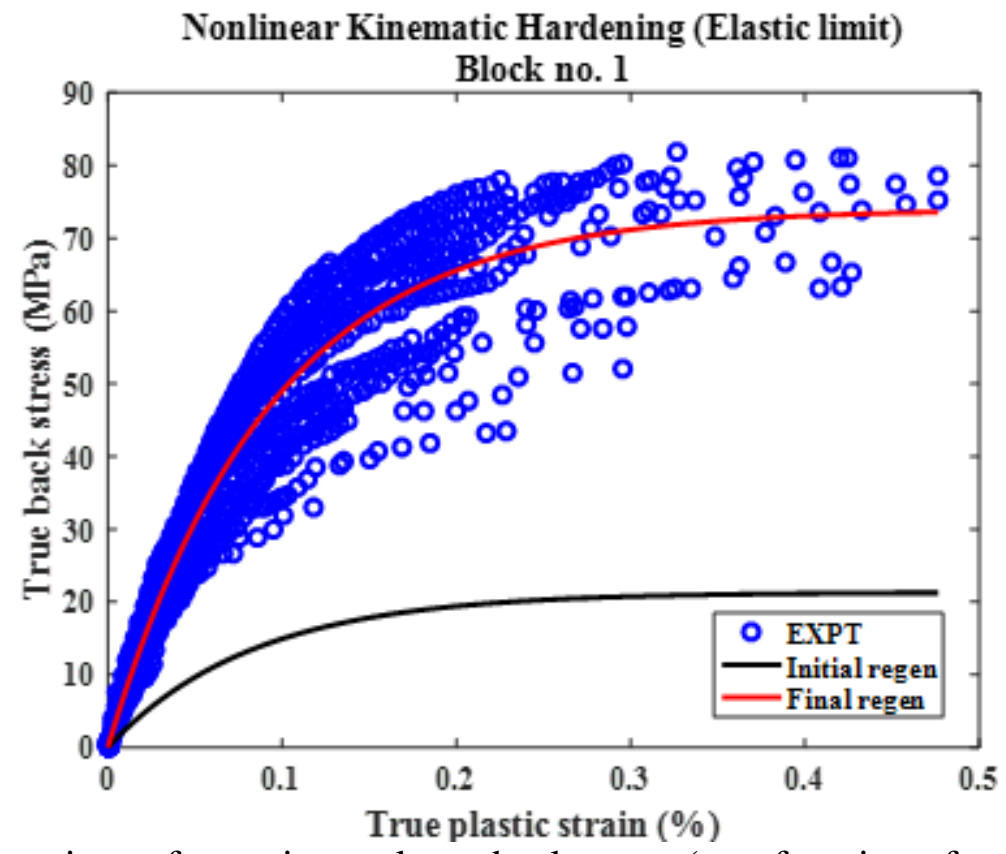

Figure 3D. 7 Comparison of experimental true back stress (as a function of true plastic strain) and predicted back stress (using nonlinear kinematic hardening equation and estimated parameters $\mathrm{C} 1 \mathrm{el}$ and $\gamma 1 \mathrm{el}$, considering elastic limit stress as yield stress) for block-1. Solid line: prediction; Circles: experimental data from 12 fatigue cycles of block-1.

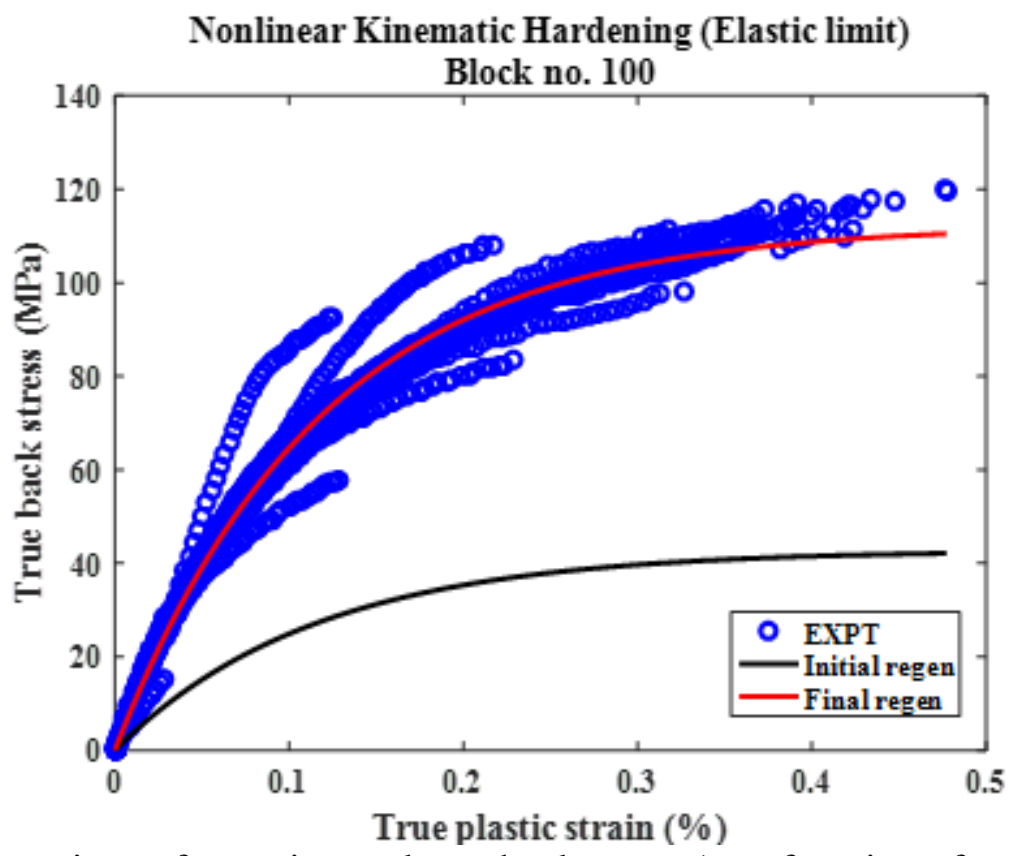

Figure 3D. 8 Comparison of experimental true back stress (as a function of true plastic strain) and predicted back stress (using nonlinear kinematic hardening equation and estimated parameters $\mathrm{C} 1 \mathrm{el}$ and $\gamma 1 \mathrm{el}$, considering elastic limit stress as yield stress) for block-100. Solid line: prediction; Circles: experimental data from 12 fatigue cycles of block-100. 


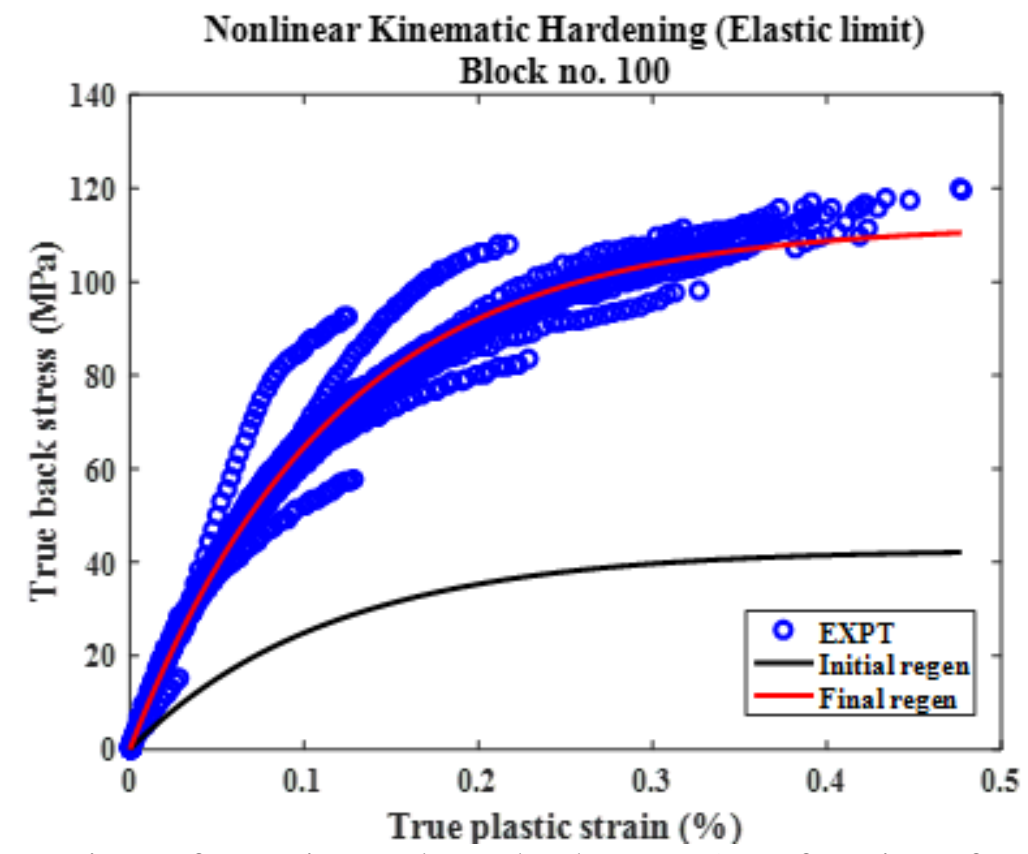

Figure 3D. 9 Comparison of experimental true back stress (as a function of true plastic strain) and predicted back stress (using nonlinear kinematic hardening equation and estimated parameters $\mathrm{C} 1 \mathrm{el}$ and $\gamma 1 \mathrm{el}$, considering elastic limit stress as yield stress) for block-1000. Solid line: prediction; Circles: experimental data from 12 fatigue cycles of block-1000.

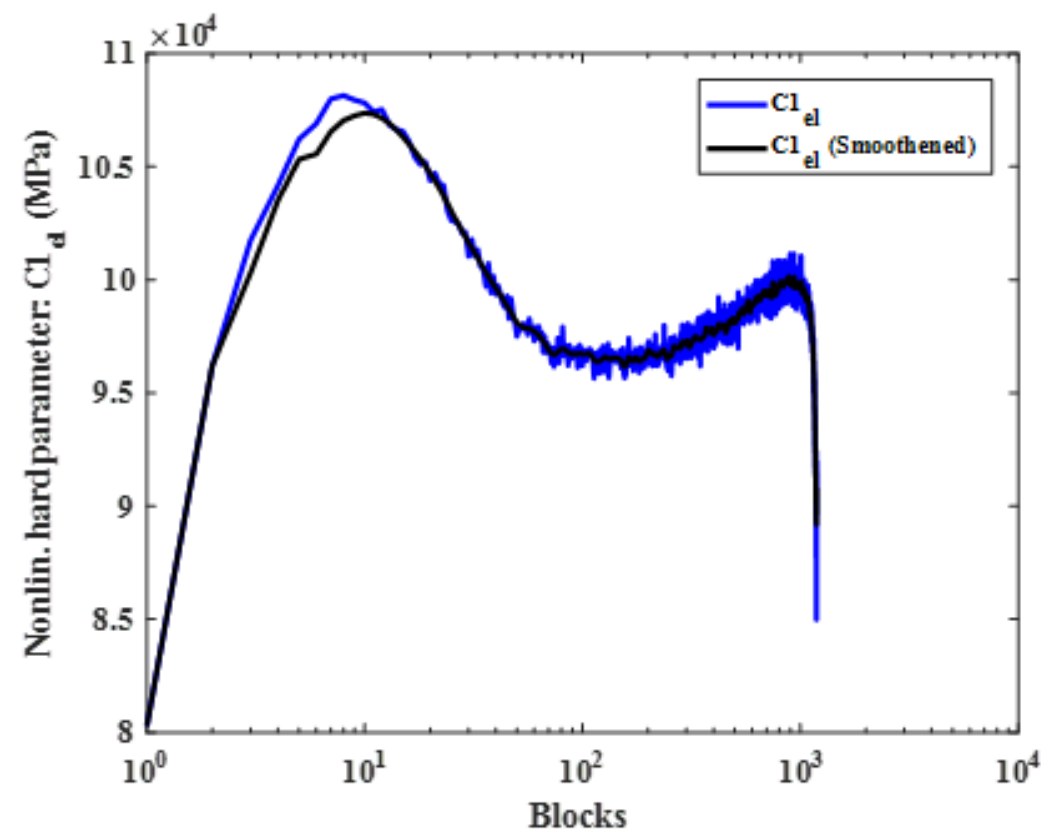

Figure 3D. 10 Block-by-block estimated nonlinear kinematic hardening or Chaboche model parameters $\left(\mathrm{C} 1_{\mathrm{el}}\right)$ for ET-F38 fatigue test, considering elastic limit stress as yield stress. 


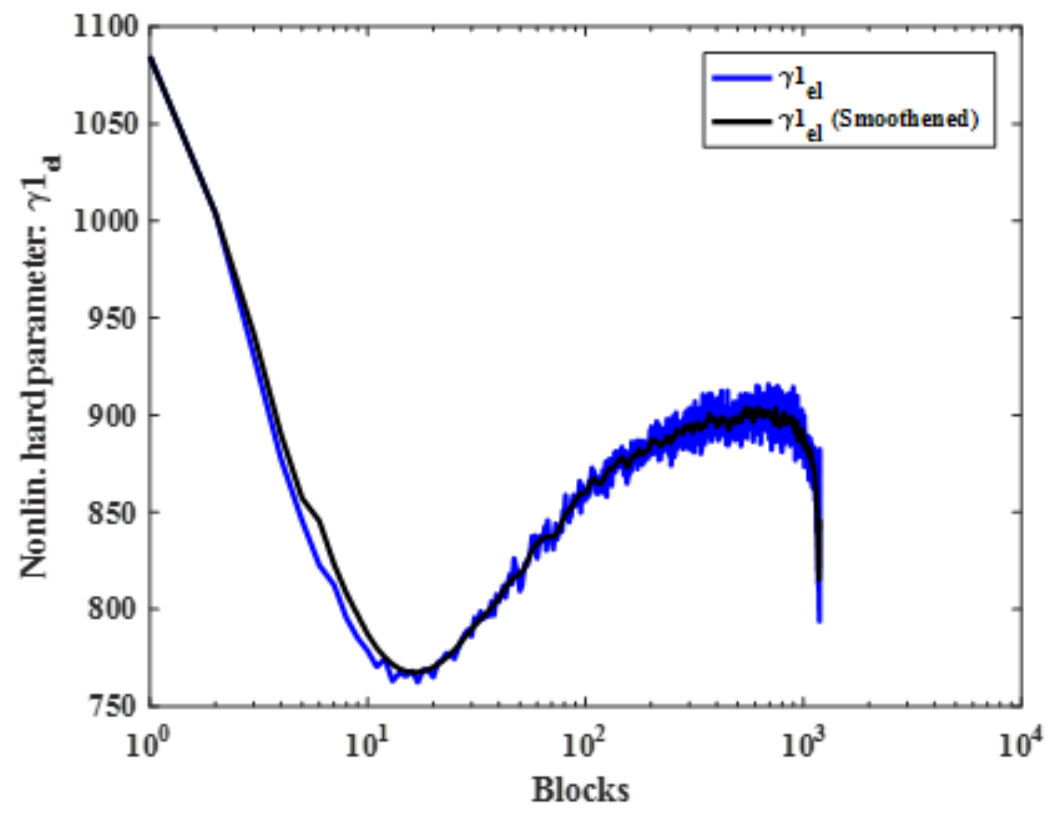

Figure 3D. 11 Block-by-block estimated nonlinear kinematic hardening or Chaboche model parameters $\left(\gamma 1_{\mathrm{el}}\right)$ for ET-F38 fatigue test, considering elastic limit stress as yield stress.

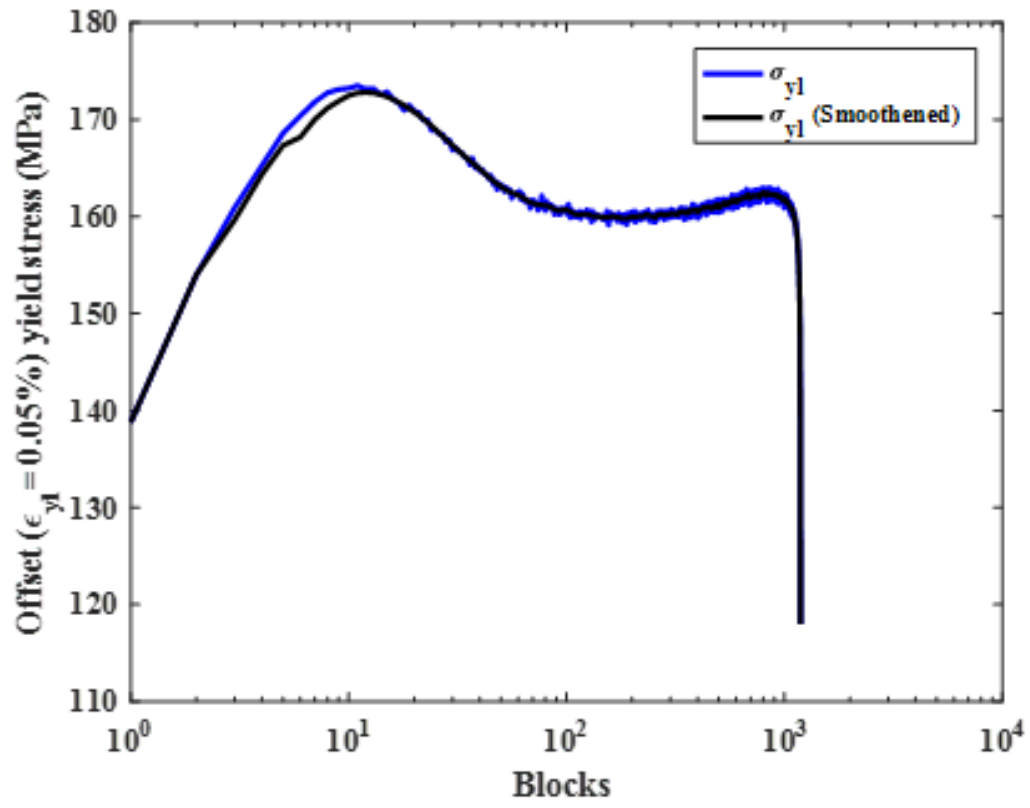

Figure 3D. 12 Block-by-block estimated stress at 0.05\% offset yield limit for ET-F38 fatigue test. 


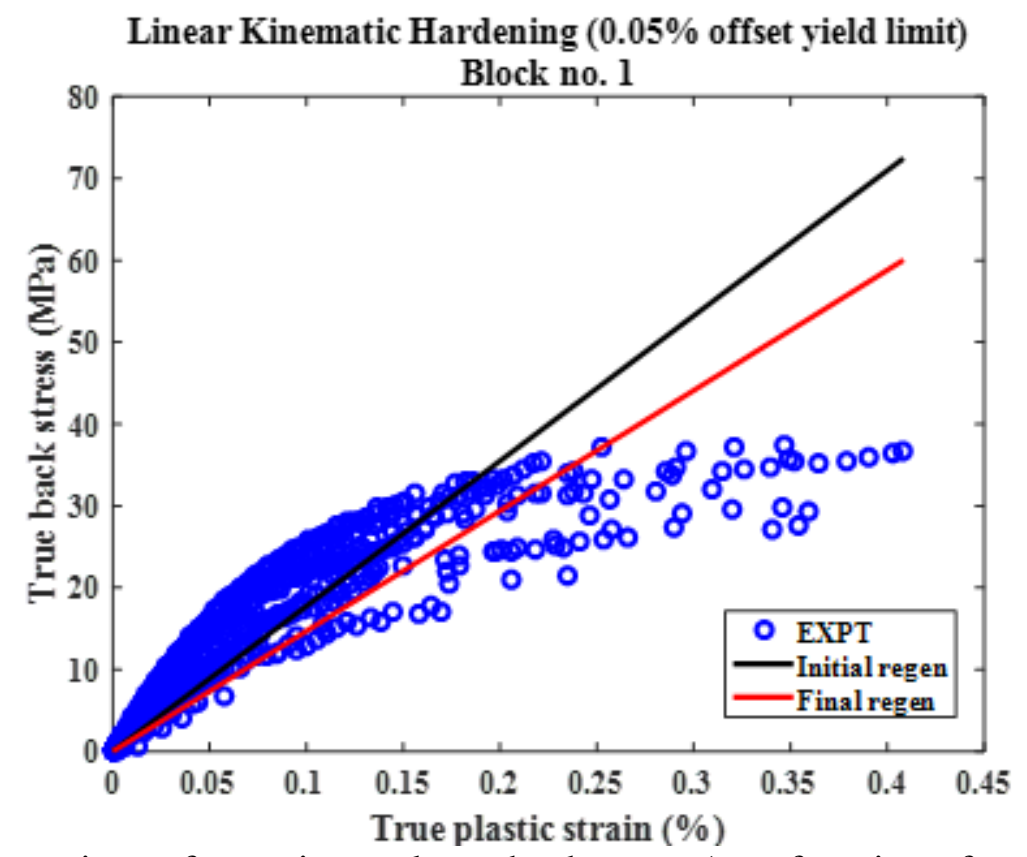

Figure 3D. 13 Comparison of experimental true back stress (as a function of true plastic strain) and predicted back stress (using linear kinematic hardening equation and estimated parameter $\mathrm{C} 1$, considering stress at $0.05 \%$ offset strain as yield stress) for block-1. Solid line: prediction; Circles:

experimental data from 12 fatigue cycles of block-1. Note: Bad prediction and, therefore, linear kinematic hardening parameter $\mathrm{C}_{\mathrm{yl}}$ based on $0.05 \%$ offset yield stress should not be used for modeling fatigue behavior of $316 \mathrm{SS}$.

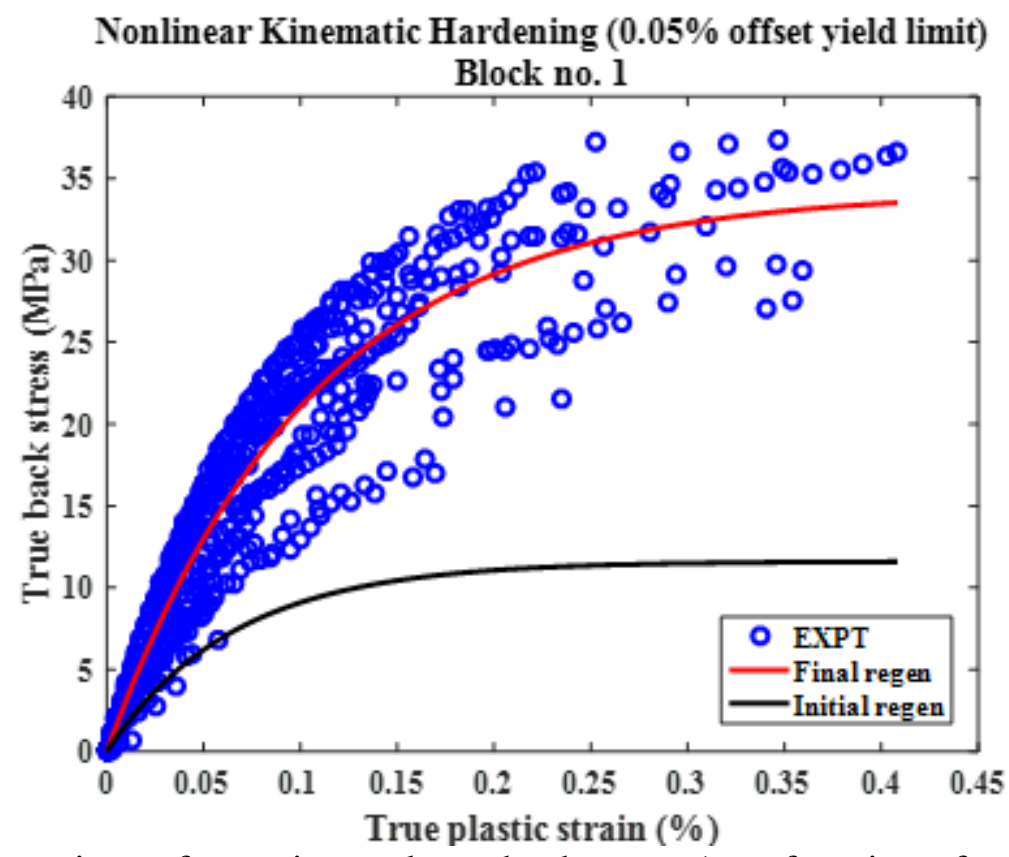

Figure 3D. 14 Comparison of experimental true back stress (as a function of true plastic strain) and predicted back stress (using nonlinear kinematic hardening equation and estimated parameters $\mathrm{C} 1_{\mathrm{yl}}$ and $\gamma 1_{\mathrm{yl}}$, considering stress at $0.05 \%$ offset strain as yield stress) for block-1. Solid line: prediction; Circles: experimental data from 12 fatigue cycles of block-1. 


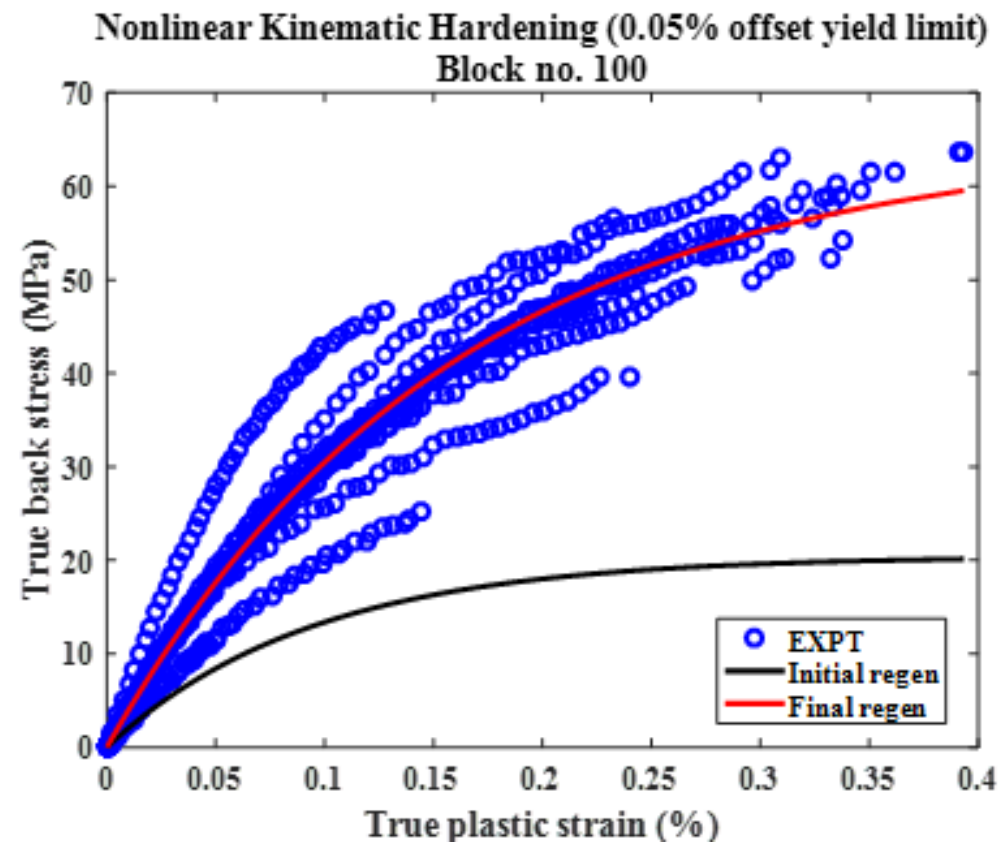

Figure 3D. 15 Comparison of experimental true back stress (as a function of true plastic strain) and predicted back stress (using nonlinear kinematic hardening equation and estimated parameters $\mathrm{C} 1_{\mathrm{yl}}$ and $\gamma 1_{\mathrm{yl}}$, considering stress at $0.05 \%$ offset strain as yield stress) for block-100. Solid line: prediction; Circles: experimental data from 12 fatigue cycles of block-100.

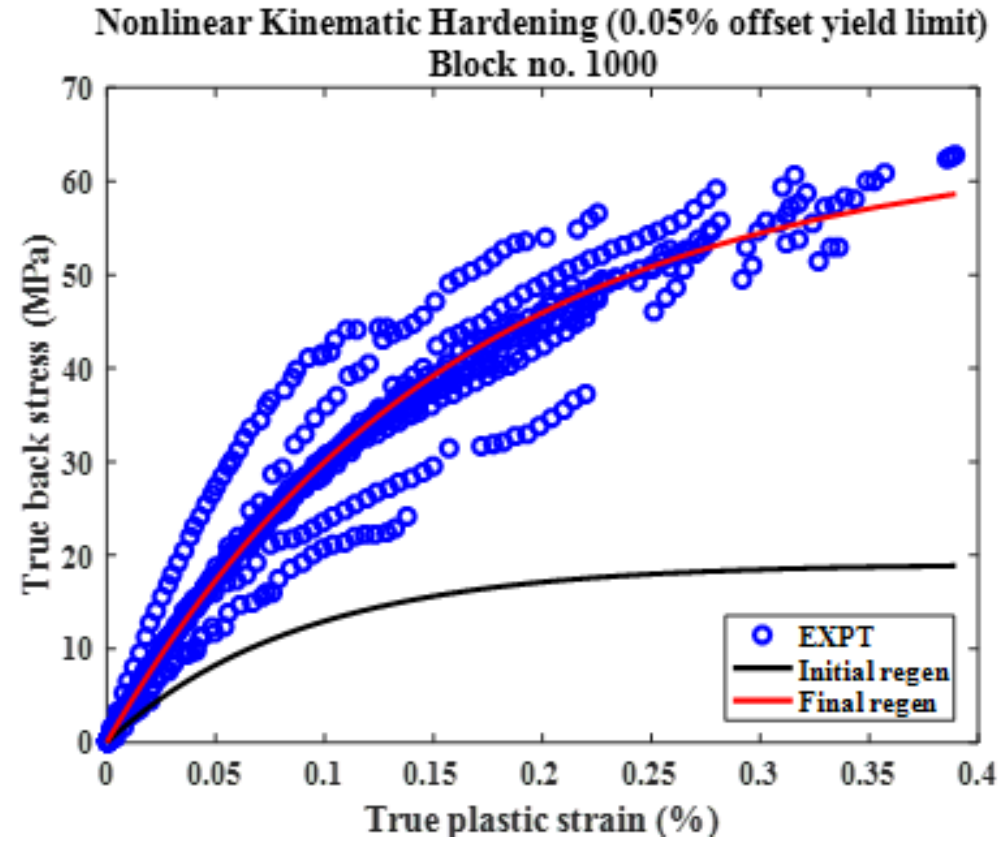

Figure 3D. 16 Comparison of experimental true back stress (as a function of true plastic strain) and predicted back stress (using nonlinear kinematic hardening equation and estimated parameters $\mathrm{C} 1_{\mathrm{yl}}$ and $\gamma 1_{\mathrm{yl}}$, considering stress at $0.05 \%$ offset strain as yield stress) for block-1000. Solid line: prediction;

Circles: experimental data from 12 fatigue cycles of block-1000. 


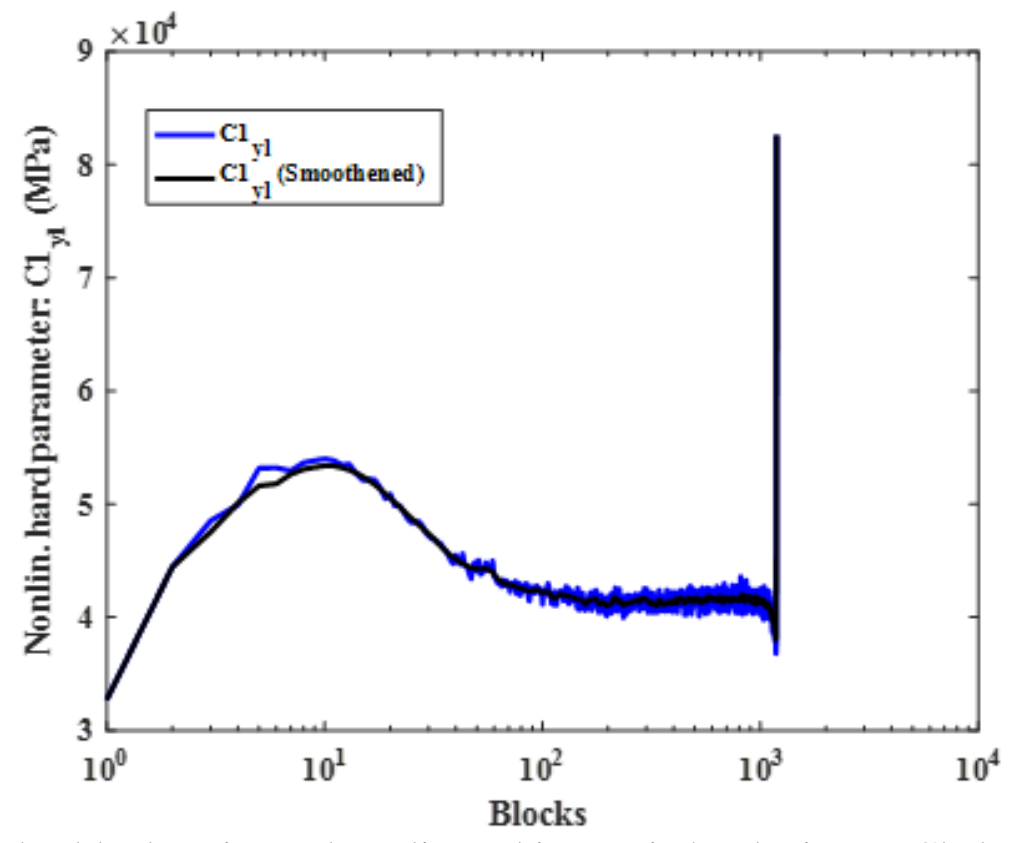

Figure 3D. 17 Block-by-block estimated nonlinear kinematic hardening or Chaboche model parameters $\left(\mathrm{C} 1_{\mathrm{yl}}\right)$ for ET-F38 fatigue test, considering stress at $0.05 \%$ offset strain as yield stress.

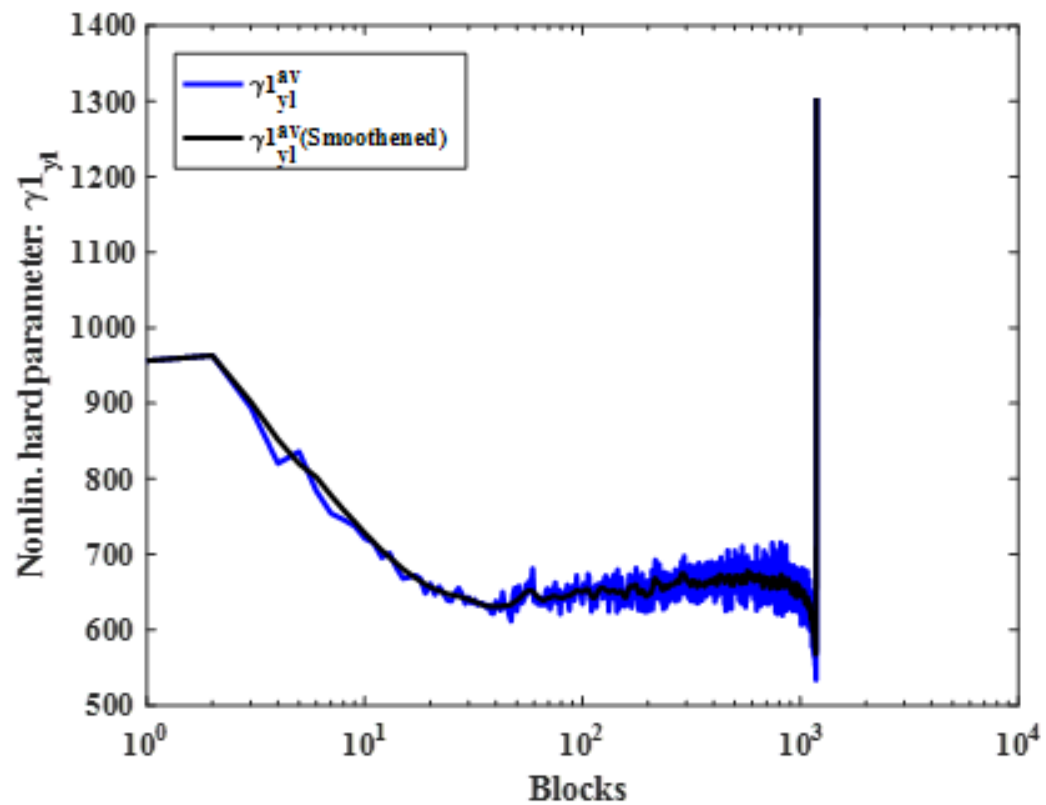

Figure 3D. 18 Block-by-block estimated nonlinear kinematic hardening or Chaboche model parameters $\left(\gamma 1_{\mathrm{yl}}\right)$ for ET-F38 fatigue test, considering stress at $0.05 \%$ offset strain as yield stress. 


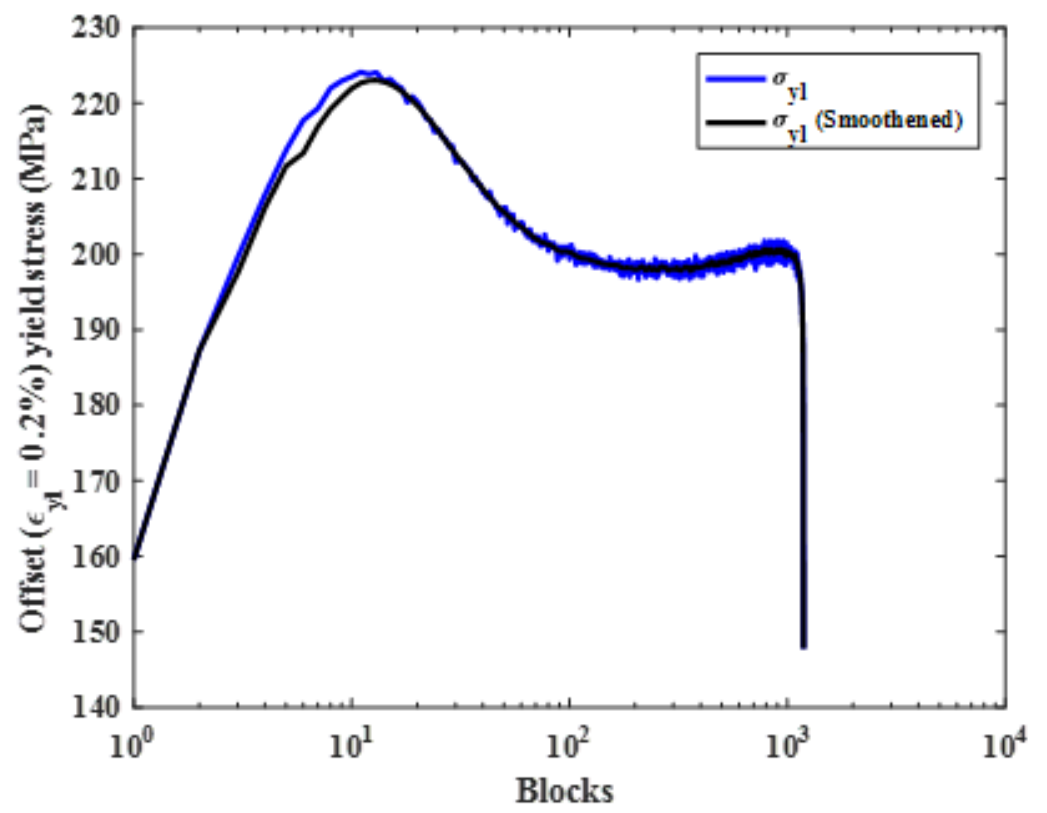

Figure 3D. 19 Block-by-block estimated stress at 0.2\% offset yield limit for ET-F38 fatigue test.

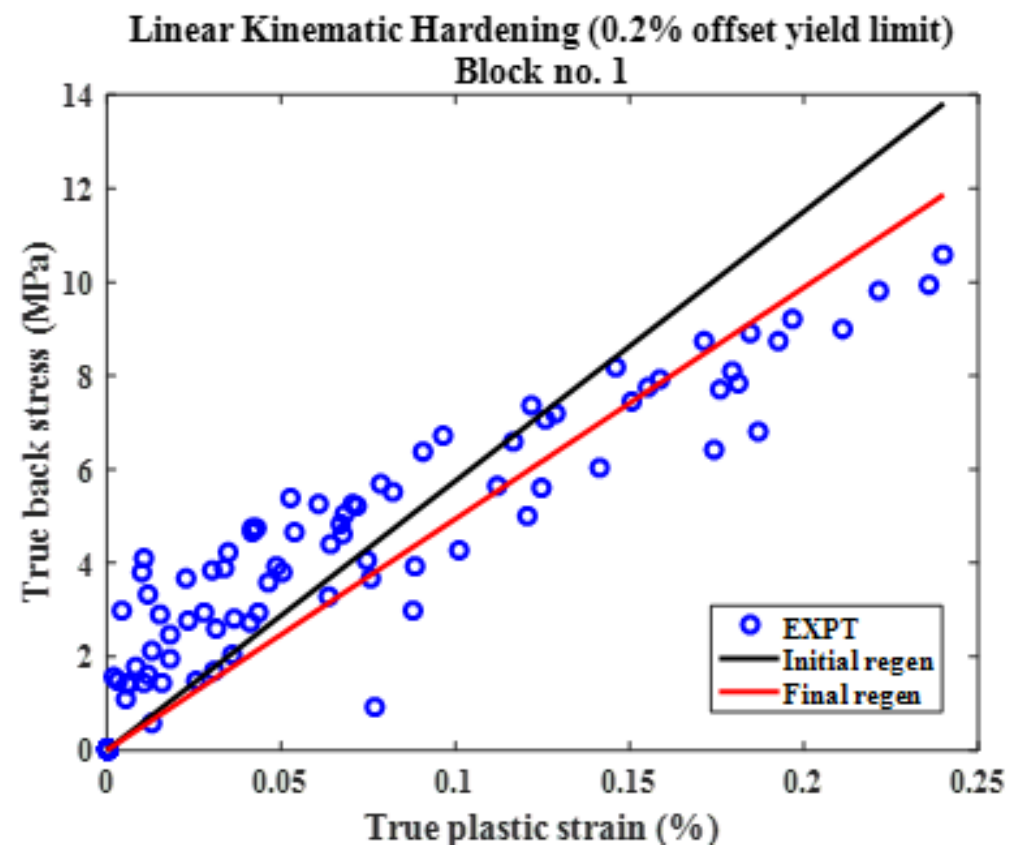

Figure 3D. 20 Comparison of experimental true back stress (as a function of true plastic strain) and predicted back stress (using linear kinematic hardening equation and estimated parameter $\mathrm{C} 1_{\mathrm{yl}}$, considering stress at $0.2 \%$ offset strain as yield stress) for block-1. Solid line: prediction; Circles: experimental data from 12 fatigue cycles of block-1. 


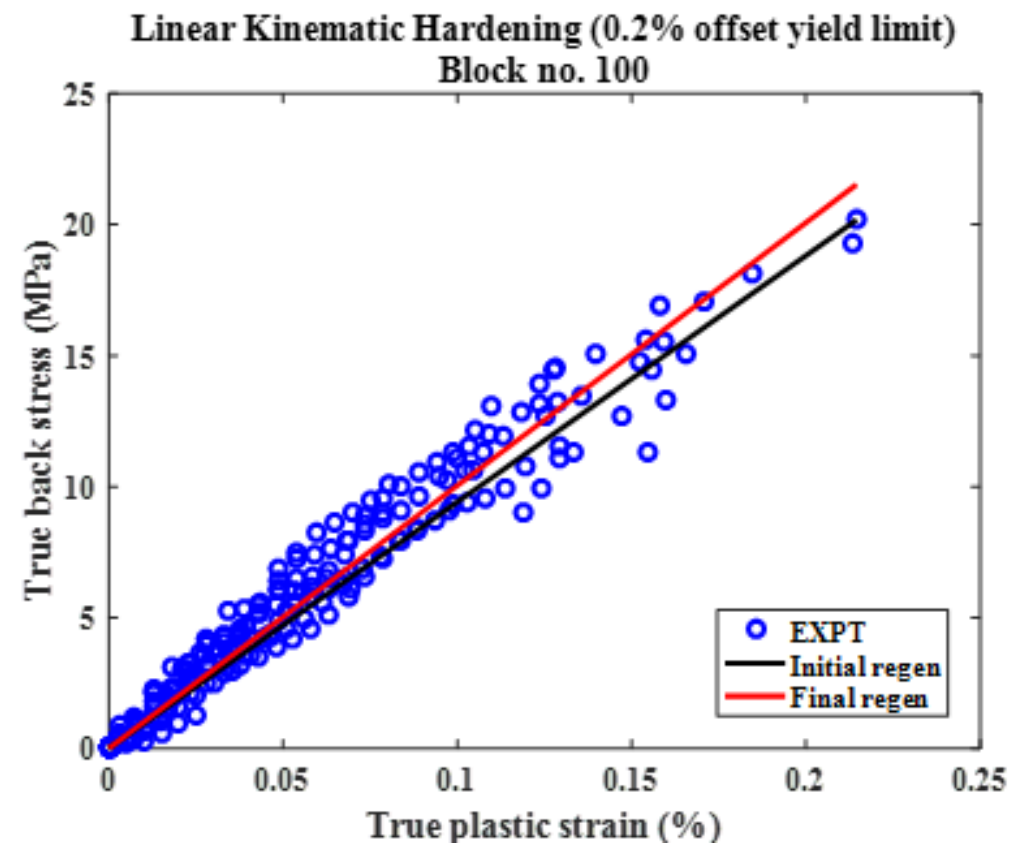

Figure 3D. 21 Comparison of experimental true back stress (as a function of true plastic strain) and predicted back stress (using linear kinematic hardening equation and estimated parameter $\mathrm{C}_{\mathrm{yl}}$, considering stress at $0.2 \%$ offset strain as yield stress) for block-100. Solid line: prediction; Circles: experimental data from 12 fatigue cycles of block-100.

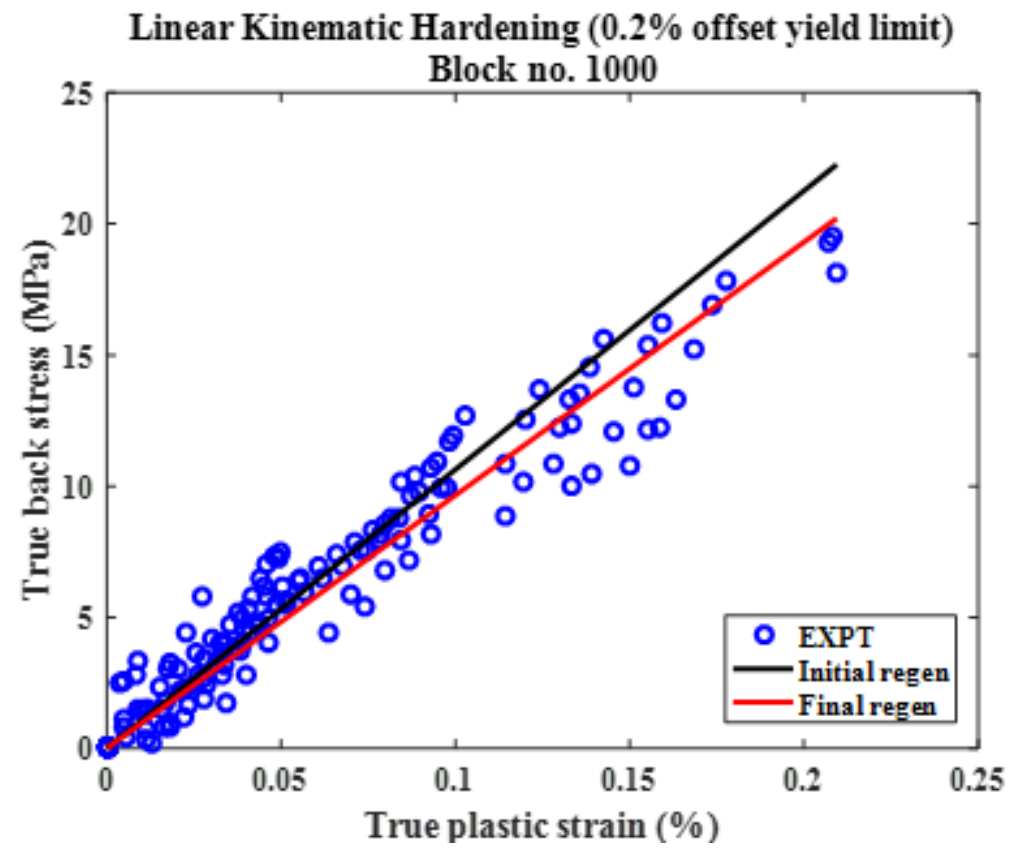

Figure 3D. 22 Comparison of experimental true back stress (as a function of true plastic strain) and predicted back stress (using linear kinematic hardening equation and estimated parameter $\mathrm{C}_{\mathrm{yl}}$, considering stress at $0.2 \%$ offset strain as yield stress) for block-1000. Solid line: prediction; Circles: experimental data from 12 fatigue cycles of block-1000. 


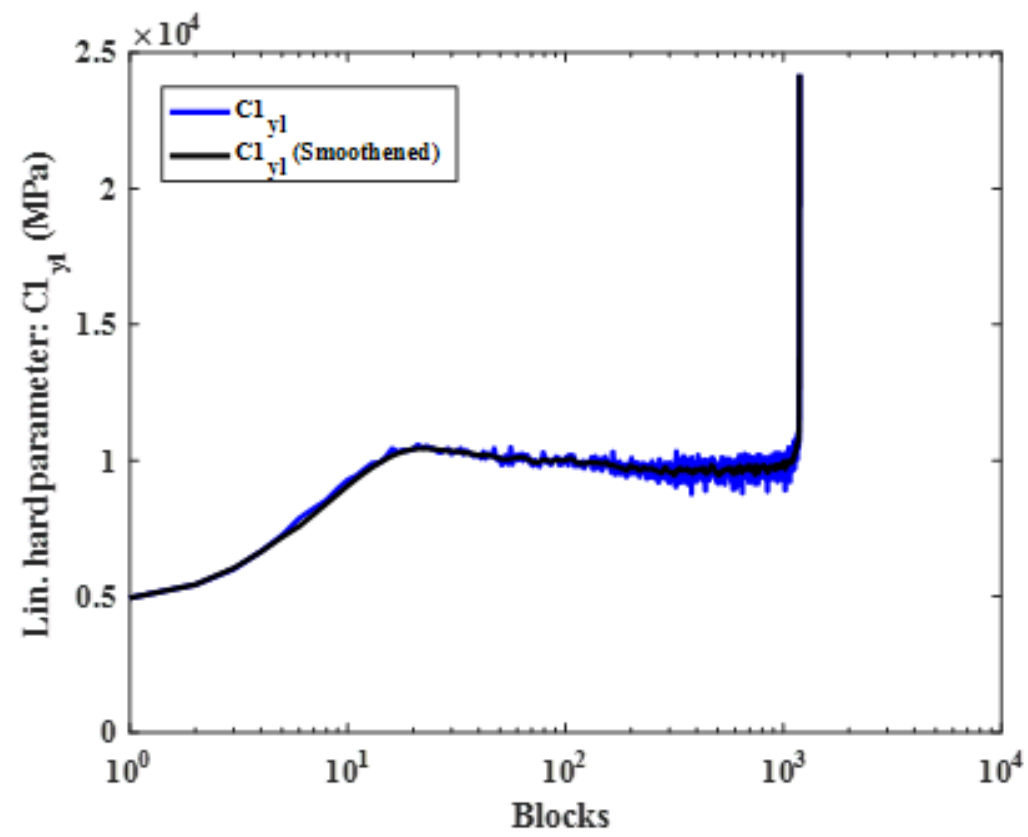

Figure 3D. 23 Block-by-block estimated linear kinematic hardening or Chaboche model parameters $\left(\mathrm{C}_{\mathrm{yl}}\right)$ for ET-F38 fatigue test, considering stress at $0.2 \%$ offset strain as yield stress.

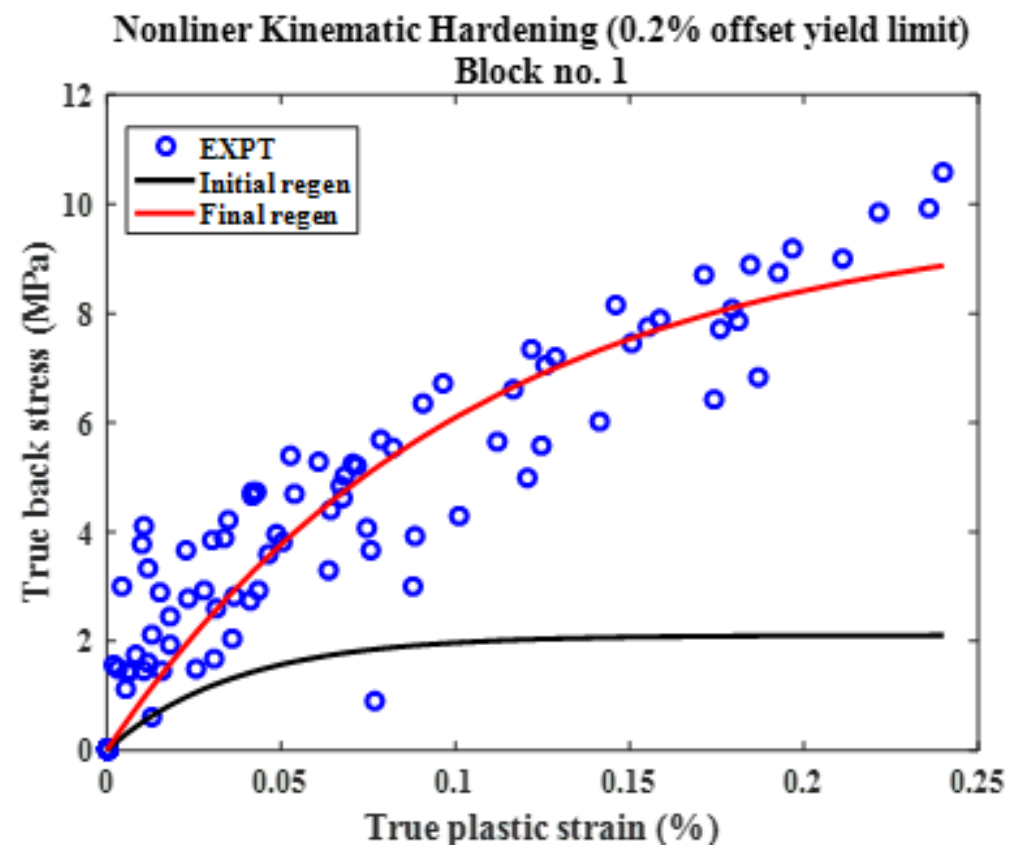

Figure 3D. 24 Comparison of experimental true back stress (as a function of true plastic strain) and predicted back stress (using nonlinear kinematic hardening equation and estimated parameters $\mathrm{C} 1_{\mathrm{yl}}$ and $\gamma 1_{\mathrm{yl}}$, considering stress at $0.2 \%$ offset strain as yield stress) for block-1000. Solid line: prediction; Circles: experimental data from 12 fatigue cycles of block-1000. 


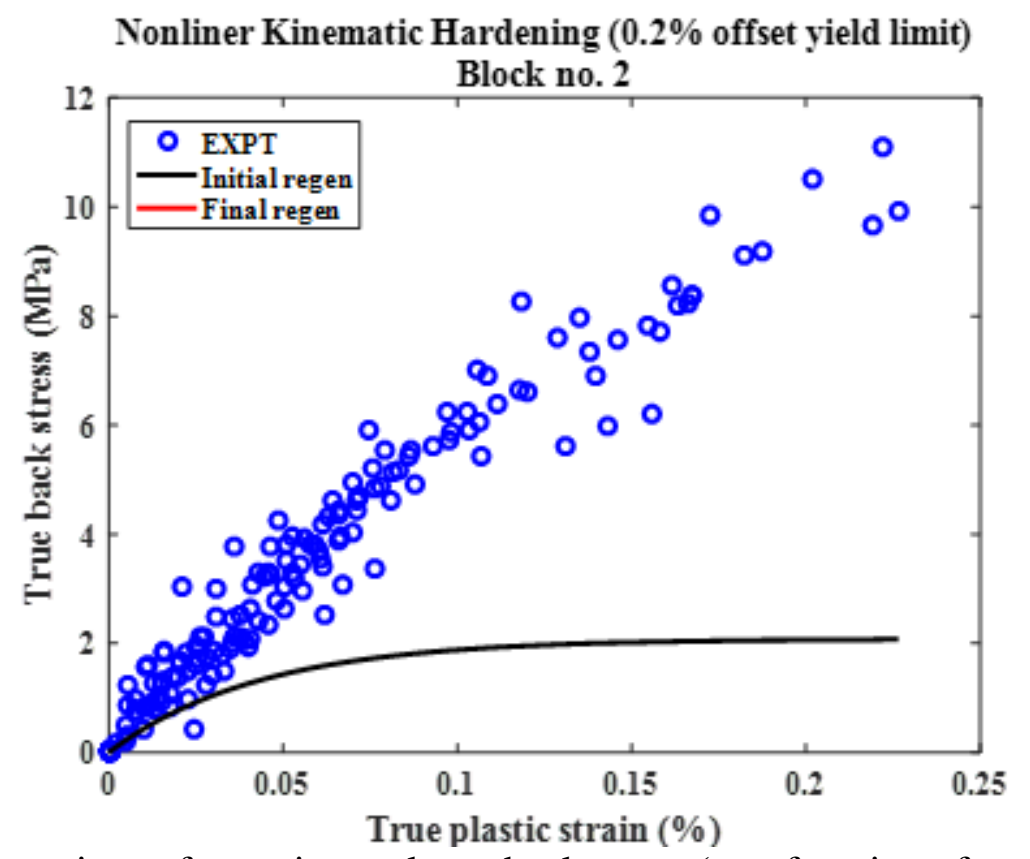

Figure 3D. 25 Comparison of experimental true back stress (as a function of true plastic strain) and predicted back stress (using nonlinear kinematic hardening equation and estimated parameters $\mathrm{C} 1_{\mathrm{yl}}$ and $\gamma 1_{\mathrm{yl}}$, considering stress at $0.2 \%$ offset strain as yield stress) for block-2. Solid line: prediction; Circles:

experimental data from 12 fatigue cycles of block-2. Note: iteration norm did not converge and, therefore, nonlinear kinematic hardening parameters $\left(\mathrm{C} 1_{\mathrm{yl}}, \gamma 1_{\mathrm{yl}}\right)$ based on $0.2 \%$ offset yield stress should not be used for modeling fatigue behavior of $316 \mathrm{SS}$. 
Appendix-3E: Supplementary Results for ET-F40 Fatigue Test

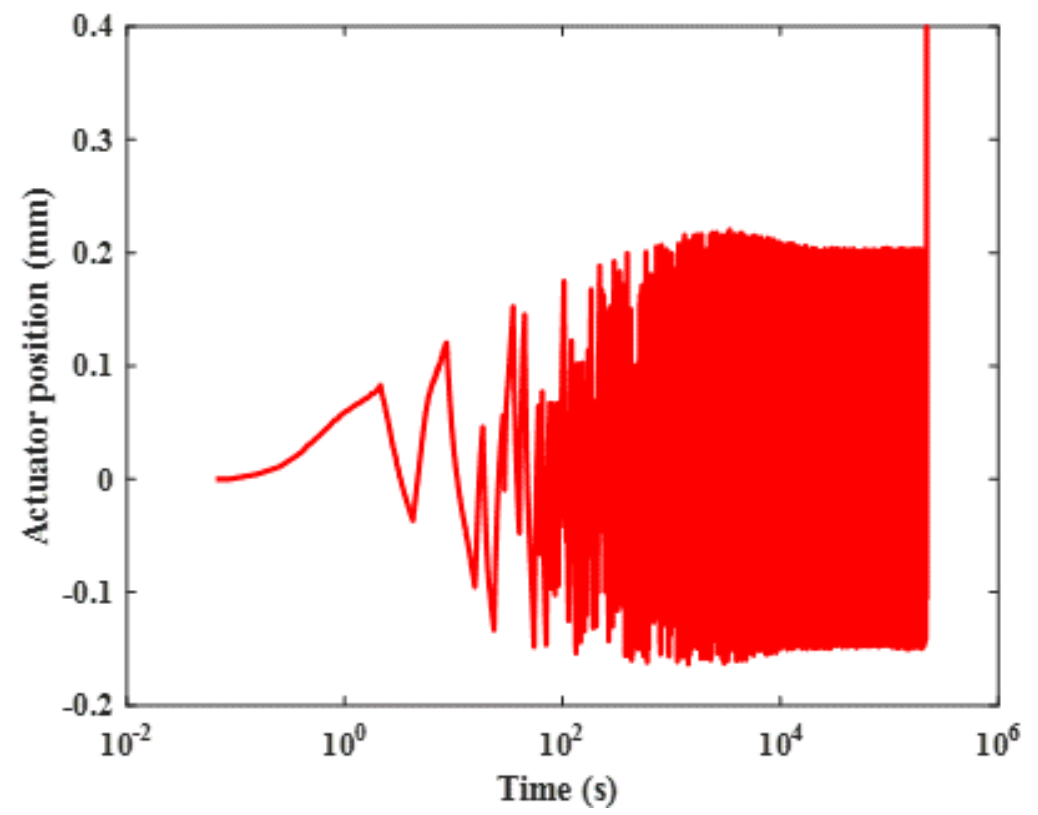

Figure 3E. 1 Observed actuator position during ET-F40. Block period $=4251 \mathrm{~s}$.

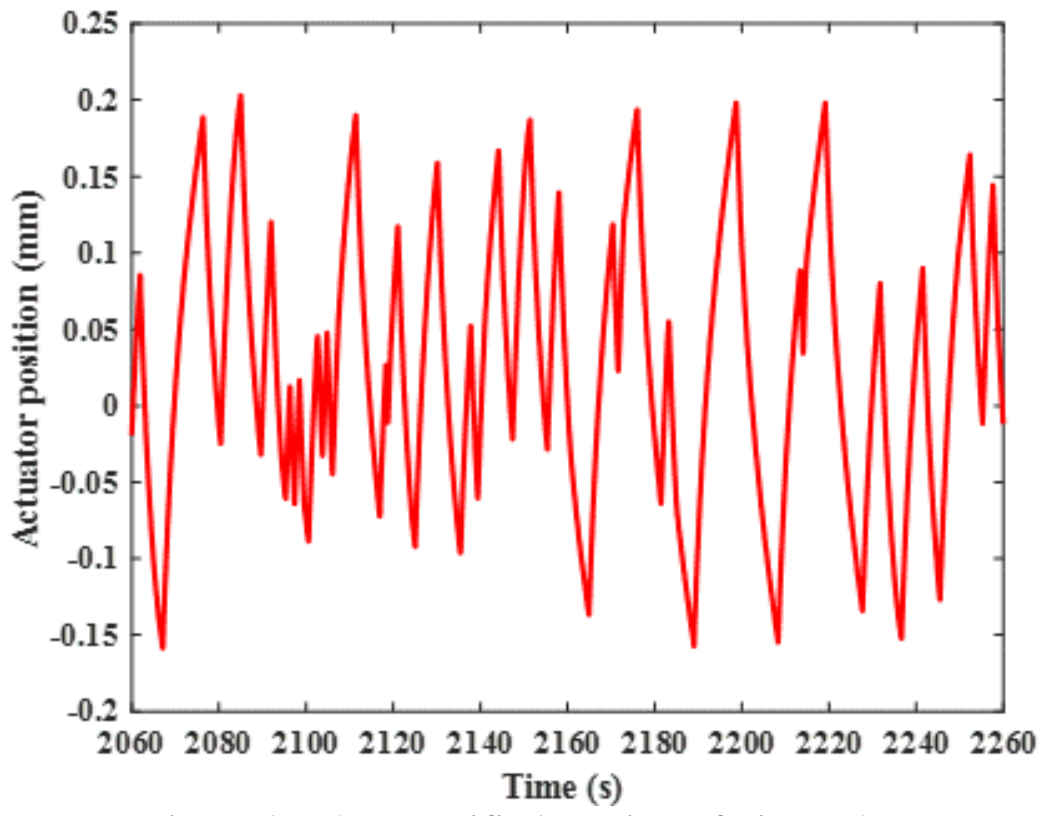

Figure 3E. 2 Magnified version of Figure 3E. 1. 


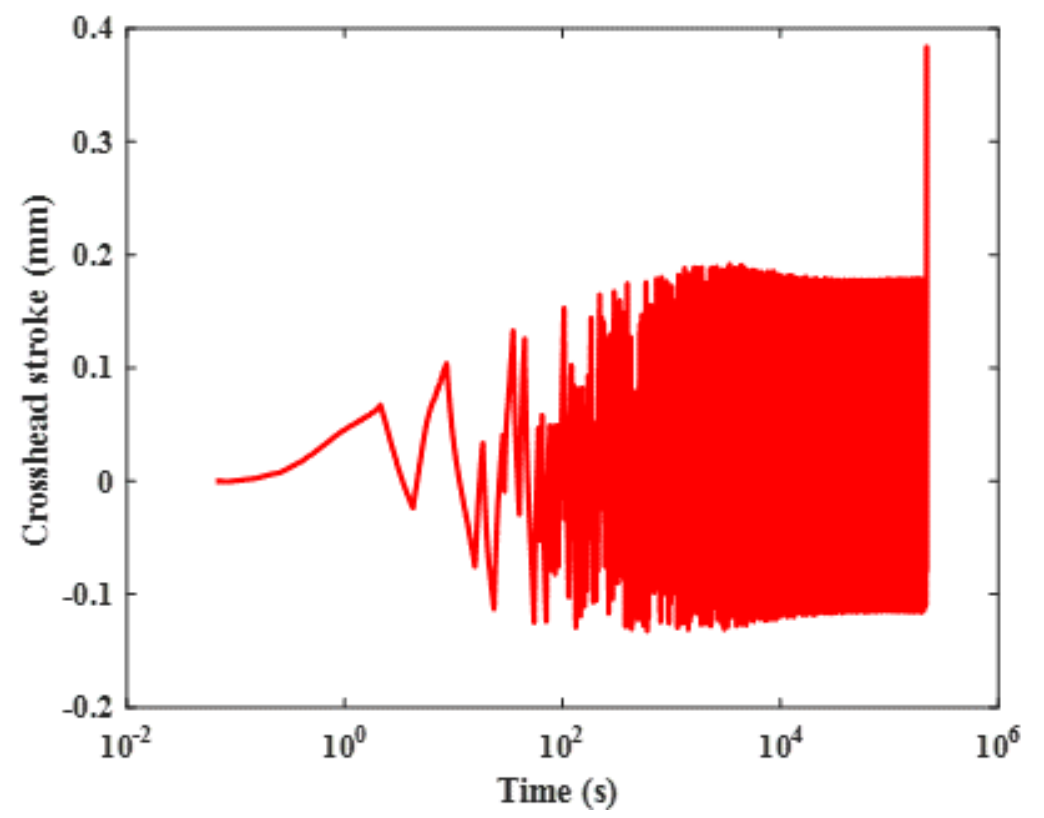

Figure 3E. 3 Observed stroke during ET-F40. Block period $=4251 \mathrm{~s}$.

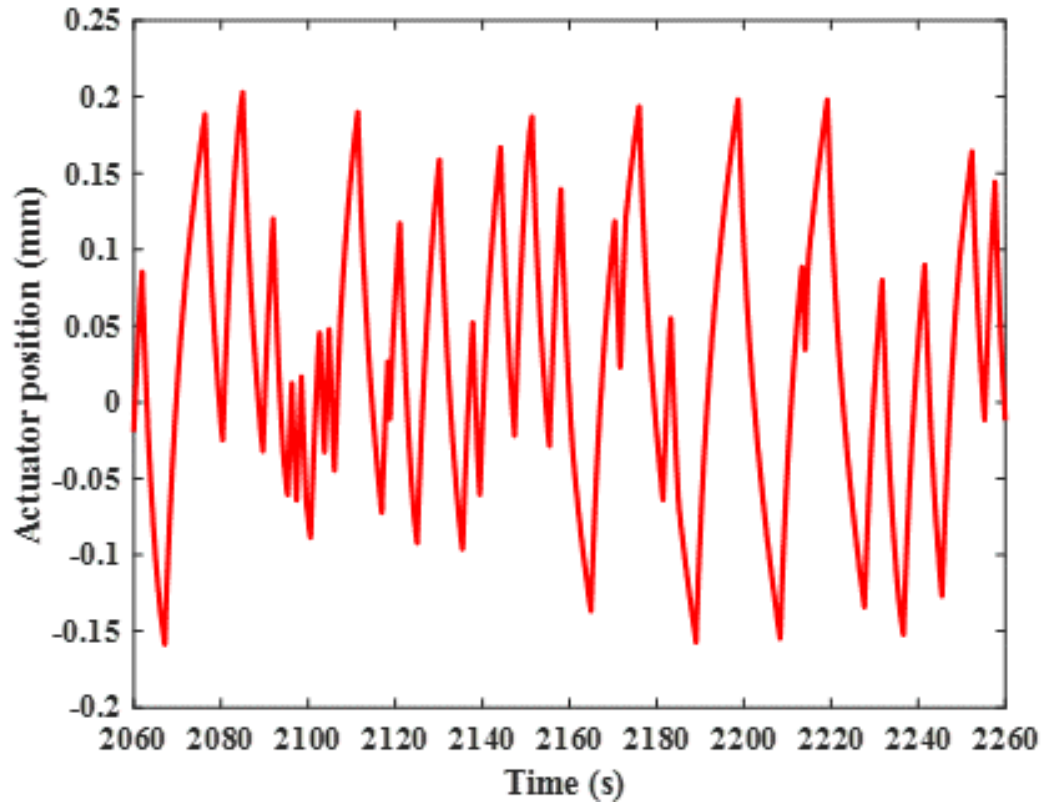

Figure 3E. 4 Magnified version of Figure 3E. 3. 
This page intentionally left blank 


\section{Argonne}

Nuclear Engineering Division

Argonne National Laboratory

9700 South Cass Avenue, BIdg. 208

Argonne, IL 60439

www.anl.gov 